\title{
The Effects of Internal Molecular Dynamics on the Evaporation/Condensation of $\boldsymbol{n}$-Dodecane
}

\author{
Rasoul Nasiri ${ }^{1}$, Vladimir M. Gun’ko ${ }^{2,1}$, Sergei S. Sazhin ${ }^{1, *}$ \\ ${ }^{1}$ Sir Harry Ricardo Laboratories, Centre for Automotive Engineering, School of Computing, \\ Engineering and Mathematics, Faculty of Science and Engineering, University of Brighton, \\ Brighton BN2 4GJ, UK \\ ${ }^{2}$ Chuiko Institute of Surface Chemistry, 17 General Naumov Street, Kiev 03164, Ukraine
}

The effects of conformerisation and internal molecular dynamics (IMD) of $n$-dodecane conformers on energy transfers between gas and liquid phases are investigated. Bond energies, Gibbs free energies of internal dynamics of a set of $n$-dodecane conformers, and energies of the molecules colliding with the surface of an $n$-dodecane nanodroplet are studied using quantum chemical calculations (DFT with $\omega$ B97X-D/cc-pVTZ and semi-empirical PM7) and ReaxFF method. The results of the analysis show that the accuracy of the methods increases as we move from the application of PM7 to the application of ReaxFF and then to DFT. Different temperature dependencies of internal Gibbs free energies of conformers in the gas and liquid phases are expected to affect the heat and mass transfer processes between them. The calculations for the gas and liquid (using the quantum solvation model; SMD) phases show significant differences in the internal dynamics of conformers and demonstrate an entropy-enthalpy competition in the evaporation/condensation of an ensemble of the conformers.

* e-mail: $\underline{\text { S.Sazhin@brighton.ac.uk }}$ 


\section{Introduction}

The study of evaporation/condensation processes is important in science ${ }^{1,2}$ and engineering. ${ }^{3}$ Characterisation of these processes can be a delicate and sophisticated task, since it requires consideration at various scales ranging from nano (atomic level in molecular dynamics (MD) applied to local interfacial phenomena) to micro (typical scale in fluid dynamics (FD) models of evaporation processes). ${ }^{4,5}$ During the heating/cooling and evaporation/condensation processes, heat and mass transfers take place simultaneously and these are controlled by the thermal evaporation/condensation coefficient (ECC) $\left(\chi_{T}\right)$ and mass ECC $\left(\beta_{m}\right){ }^{6,7}$ Although there have been many theoretical and experimental studies of $\beta_{m}$ for various substances (e.g. water, ${ }^{8-12} n$-alkanes, ${ }^{13-15}$ diethylene glycol ${ }^{16}$ ) and of $\chi_{T},{ }^{6,7,17,18}$ the underlying physics of the processes is still not fully understood. For example, Varilly and Chandler ${ }^{8}$ estimated the $\beta_{m}$ of water to be close to unity using the simple point charge (extended) (SPC/E) solvent model and transition path sampling technique. Other authors ${ }^{9,10}$ obtained the values of $\beta_{m}$ equal to 0.25 and 0.868 using the SPC/E and three-site transferrable intermolecular potential (TIP3P) solvent models, respectively. All the aforementioned values were obtained using MD simulations at $298 \mathrm{~K}$. The experimentally observed values of $\beta_{m}$ were reported to be $0.62 \pm 0.09$ (Raman spectroscopy) $^{11}$ and 0.25 to 1.0 (Tensimeter Jet) $^{12}$. Xia and Landman ${ }^{13}$ predicted a constant value (0.9) of $\beta_{m}$ for hexane and hexadecane molecules using an empirical potential at 334-554 K. Cao et al. ${ }^{14}$ and Xia et al. ${ }^{15}$ obtained the values of $\beta_{m}$ for $n$ dodecane using a modified OPLS (Optimised Potentials for Liquid Simulations) force field. It was shown that this coefficient decreases from 0.9 to 0.3 when $T / T_{\mathrm{c}}$ increases from 0.45 to 0.9 , where $T_{c}=658.2 \mathrm{~K}$ is the critical temperature for $n$-dodecane. A certain scatter in the published values of $\beta_{m}$ can be caused by complexity of the interfacial phenomena simplified in different ways in the above-mentioned approaches. 
Thermal effects on the evaporation/condensation processes, associated with vibrational, rotational and conformational dynamics in flexible molecules (e.g. $n$-dodecane) at the interface of nanodroplets, have not been investigated. The most accurate results would be expected if these effects were studied based on ab initio calculations. ${ }^{19,20}$ Recently, it was established by high-level $a b$ initio calculations that dihydrogen interactions $(\mathrm{C}-\mathrm{H} \cdots \mathrm{H}-\mathrm{C})$ in alkanes depend on both bond orientations and intermolecular distances. ${ }^{21}$ While conventional potentials express the interaction energy as a function of the bond distance, the contributions of non-bonding interatomic energy terms (vdW and Coulomb) in the reactive force field ReaxFF $^{22}$ are shielded at close distances between atoms (in chemical bonds). The bonding contributions are calculated using the bond order (bond length) of pairs of atoms for various chemical bonds. Atomic charges are updated in a self-consistent way using the electron equilibration method. ${ }^{22}$ The ReaxFF model has been successfully applied to study combustion and pyrolysis of $n$-alkanes, ${ }^{22-25}$ aromatics, $^{22,26}$ and other hydrocarbons. ${ }^{27,28} \mathrm{We}$ can then anticipate that the comparison of the performance of ReaxFF with density functional theory $(\omega \mathrm{B} 97 \mathrm{X}-\mathrm{D})^{30}$ and semi-empirical $(\mathrm{PM} 7)^{31}$ results can allow us to establish whether ReaxFF $^{22,23}$ can be reliable to study the evaporation/condensation processes.

The main aim of this work is to study the effects of conformerisation ${ }^{29}$ (multiple structures induced by internal molecular dynamics effects) on the difference between internal Gibbs free energies of $n$-dodecane in the gas and liquid phases. The results for individual conformers ( $G_{\text {conf-rovib }}^{\text {gas } j}$ and $G_{\text {conf-rovib }}^{\text {liq, }}$ ) and a set of conformers ( $G_{\text {conf-rovib }}^{\text {gas }}$ and $\left.G_{c o n f-r o v i b}^{\text {liq }}\right)$ will be compared ('conf-rovib’ refers to conformational, rotational and vibrational dynamics). The reliability of the bond energy bond order (BEBO) approach of ReaxFF ${ }^{22,23}$ and semiempirical $\mathrm{PM} 7^{31}$ methods will be assessed based on the results of calculations of bond energies, internal Gibbs free energies of a set of $n$-dodecane conformers, and energies of molecules colliding with the surface of a nanodroplet. The results will be compared with 
available experimental data (e.g. bond energy and normal mode analysis) and those calculated using $\omega$ B97X-D/cc-pVTZ ${ }^{30}$ electronic model chemistry. The quantum solvation model SMD, with emphasis on the comparison of the entropy-enthalpy components of internal Gibbs free energies in the evaporation/condensation processes, will be used in our analysis. As in the previous studies, ${ }^{14,15,32} n$-dodecane is used as a representative of Diesel fuel.

\section{Theory and Computational Methods}

2-1 Density Functional Theory Calculations. 95 conformers of $n$-dodecane were fully optimised using the restricted $\omega$ B97X-D/cc-pVTZ model ${ }^{30}$ which has been recently established to provide geometries and electronic energies close to those predicted by CCSD(T)/cc-pVTZ for $n$-alkanes. ${ }^{33}$ The Gibbs free energies of this ensemble of conformers in liquid and gas phases were shown to be in good agreement with experimental results. Therefore, we studied this number of conformers to explore internal molecular dynamics effects during the evaporation/condensation processes (see Section 3-4 for more detail). Stand-alone code ConfGen ${ }^{34}$ generated all conformers based on the specifications of internal rotating $\mathrm{C}-\mathrm{C}$ bonds. Vibration analyses were performed for all conformers with the same functional and basis set as used during optimisation of the geometries. The vibrational frequencies were left unscaled. The calculations were carried out using the Gaussian 09 program suite. ${ }^{35}$

The values of $G_{\text {conf-rovib }}^{\text {gas, } j}$ for each conformer and $G_{\text {conf-rovib }}^{\text {gas }}$ for an ensemble of 95 conformers were determined using the energies of optimised structures and force constants. These values were obtained using multi-structural partition functions in which rotational, vibration, conformational and torsional effects were taken into account based on the following formula: 
$Q_{c o n f-r o v i b}^{M S-T}=\sum_{j=1}^{J} Q_{r o t, j} Q_{v i b, j} \exp \left(-\frac{U_{j}}{k_{B} T}\right) \times \prod_{\tau=1}^{t} \varphi_{j, \tau}$,

where $k_{\mathrm{B}}$ is Boltzmann constant, and $U_{j}$ is the energy of the $j$ th structure, $J$ is the number of conformers. Note that when $J$ is equal to 1 (one conformer), the single structure partition function is defined as $Q_{\text {rovib }}^{S S-H}$. The coefficients $\varphi_{j, \tau}$ describe the torsional anharmonicity effects. When these coefficients are equal to 1 for a certain $j, Q_{\text {conf-rovib }}^{M S-T}$ can be calculated without taking into account the effect of coupling. In this case $Q_{\text {conf -rovib }}^{M S-T}$ is known as $Q_{\text {conf-rovib }}^{M S-H}$. It is controlled by harmonic vibrational $\left(Q_{\text {vib }}\right)$ and rotational $\left(Q_{\text {rot }}\right)$ partition functions:

$$
\begin{aligned}
Q_{\text {rot }, j} & =\frac{\sqrt{\pi}}{\sigma_{\text {rot }, j}}\left(\frac{2 k_{B} T}{\hbar^{2}}\right) \sqrt{I_{A, j} I_{B, j} I_{C, j}}, \\
Q_{v i b, j} & =\prod_{i=1}^{F} \frac{\exp \left(-\frac{\hbar \omega_{j, i}}{2 k_{B} T}\right)}{1-\exp \left(-\frac{\hbar \omega_{j, i}}{k_{B} T}\right)},
\end{aligned}
$$

where $\sigma_{r o t, j} \geq 1$ is the symmetry number of the molecule, and $I_{A, j}, I_{B, j}$, and $I_{C, j}$ are principal moments of inertia. $F$ and $\omega_{j, i}$ indicate the number of degrees of freedom for vibration modes and vibration frequency of the $i^{\text {th }}$ mode of the $j^{\text {th }}$ structure, respectively (see ${ }^{34}$ for more details).

The optimised geometries and Hessians were applied to calculate the values of internal Gibbs free energy of an ensemble of $n$-dodecane conformers $\left(G_{\text {conf-rovib }}\right)$ using the following equation:

$G_{\text {conf-rovib }}=-R T \ln \left(Q_{\text {conf-rovib }}\right)+R T$

and the MSTor program for the constant pressure of $1 \mathrm{~atm}$. The temperatures rose from 300 to $650 \mathrm{~K}$ in steps of $25 \mathrm{~K}$. 
In order to estimate the internal Gibbs free energy of evaporation for each conformer ( $\Delta G_{\text {conf-rovib }}^{e v, j}$ ), the differences between the internal Gibbs free energies of $n$-dodecane conformers in the gas $\left(G_{\text {conf-rovib }}^{\text {gas } j}\right)$ and liquid $\left(G_{\text {conf-rovib }}^{\text {liq } j}\right)$ phases were calculated $\left(\Delta G_{\text {conf }- \text { rovib }}^{e v, j}=G_{\text {conf }- \text { rovib }}^{\text {gas }, j}-G_{\text {conf -rovib }}^{\text {liq,j }}\right.$ ). In the case of the gas phase, the multi-structural harmonic (MS-H) approximation was used, while the solvation method SMD ${ }^{47}$ was applied for the liquid phase. SMD is the universal continuum solvation model developed by Truhlar's group. It is based on the solute electron density, the dielectric constant and surface tensions. The temperature dependence of the surface tension was taken into account based on the following formula: ${ }^{48,51}$

$$
\gamma=A\left(1-\frac{T}{T_{c}}\right)^{n}
$$

where $A$ and $n$ are constants: $A=80.1946 * 10^{-3} \mathrm{kcal} /\left(\mathrm{mol}^{*} \AA^{2}\right), n=1.3325$, and $T_{c}$ is the critical temperature of $n$-dodecane $(658.2 \mathrm{~K})$. The self-consistent reaction field (SCRF) method, implemented in the Gaussian 09 suite, was used to take into account the effect of temperature on the surface tension, based on Equation (5). Liquid $n$-dodecane with dielectric constant $\varepsilon=$ 2.006 was used as an implicit solvent. The variations of internal Gibbs free energies of evaporation for an ensemble of 94 conformers $\left(\Delta G_{\text {conf-rovib }}^{e v}=G_{\text {conf-rovib }}^{\text {gas }}-G_{\text {conf-rovib }}^{\text {liq }}\right)$ were calculated using the optimised geometries both in gas and liquid phases. ${ }^{48}$ As follows from our analysis, there is no analogy in the liquid phase for conformer 1 in the gas phase. Hence, 94, rather than 95, conformers were used for the calculation of $\Delta G_{\text {conf-rovib }}^{e v}$ The calculations were performed up to $650 \mathrm{~K}$, which is close to the critical temperature of $n$-dodecane, although we appreciate that most $n$-dodecane molecules are expected to be in the gas phase at temperatures above the boiling temperatures (487 K - 491 K at atmospheric pressure).

The $a b$ initio molecular dynamics (AIMD) simulations were carried out using Quickstep implemented in the $\mathrm{CP}_{2} \mathrm{~K}^{36}$ program. The auxiliary density matrix method 
$(\mathrm{ADMM})^{37}$ and SZV-MOLOPT-GTH basis set were applied in order to reduce computational costs. All AIMD calculations were run in NVE ensembles in which conservation of energy was taken into account at microhartree precision. DFT trajectories were generated using the $\omega$ B97X-D exchange-correlation functional ${ }^{30}$ and Goedecker-Teter-Hutter (GTH) pseudopotentials $^{38}$ with the energy real cut-off 60 Rydberg (this determines the grid at which Gaussian is mapped), and cut-off 1000 Rydberg (this controls the coarser grid). Vacuum boundary conditions have been used in three directions. Since CP2K uses the periodic boundary condition (PBC), a wavelet-based Poisson solver was applied in order to truncate the wave function at the edges of the cubic box $6 \times 6 \times 6 \mathrm{~nm}^{3}$. This allowed us to avoid any artificial effects caused by the periodic images when the size of nanodroplets was smaller than that of the box (in our case the size of the nanodrop was $5 \times 3.8 \times 4 \mathrm{~nm}^{3}$ ).

2-2 Semi-empirical calculations. The bond energies and internal Gibbs free energies of the optimised $n$-dodecane structures were also calculated using the PM7 method. The following formulae were applied for the estimation of the bond energies (BE) of $\mathrm{C}-\mathrm{H}$ and $\mathrm{C}-\mathrm{C}$ bonds: where $\Delta_{\mathrm{r}} H_{\mathrm{T}_{0}}(\mathrm{RH})$ and $\Delta_{\mathrm{r}} H_{\mathrm{T}_{0}}\left(\mathrm{R}^{\prime} \mathrm{R}^{\prime \prime}\right)$ refer to $\mathrm{BE}$ of $\mathrm{C}-\mathrm{H}$ and $\mathrm{C}-\mathrm{C}$; $\mathrm{RH}$ and $\mathrm{R}$ refer to $n$ dodecane molecules and dodecyl radicals respectively; and $\mathrm{R}^{\prime}$ or R" denote other possible alkyl molecules which can be produced via C-C bonds, $T_{0}=298.15 \mathrm{~K}$.

$$
\begin{aligned}
& \Delta_{\mathrm{r}} H_{T_{0}}(\mathrm{RH})=\Delta_{\mathrm{f}} H_{\mathrm{T}_{0}}(\mathrm{R})+\Delta_{\mathrm{f}} H_{\mathrm{T}_{0}}(\mathrm{H})-\Delta_{\mathrm{f}} H_{\mathrm{T}_{0}}(\mathrm{RH}), \\
& \Delta_{\mathrm{r}} H_{\mathrm{T}_{0}}\left(\mathrm{R}^{\prime} \mathrm{R}^{\prime \prime}\right)=\Delta_{\mathrm{f}} H_{\mathrm{T}_{0}}\left(\mathrm{R}^{\prime}\right)+\Delta_{\mathrm{f}} H_{\mathrm{T}_{0}}\left(\mathrm{R}^{\prime \prime}\right)-\Delta_{\mathrm{f}} H_{\mathrm{T}_{0}}\left(\mathrm{R}^{\prime} \mathrm{R}^{\prime \prime}\right),
\end{aligned}
$$

To study the dynamics of evaporation/condensation of molecules from/to nanodrops using PM7, the dynamic reaction coordinate (DRC) method was applied. The MOPAC2012 program suite $^{39}$ was used to study the interactions of molecules with nanodroplets for various orientations of attacking molecules relative to the nanodroplet surface, as well as various velocities of the attacking molecules. 
2-3 Reactive Force Field calculations. We used the Amsterdam Density Functional (ADF) package $^{40}$ for all ReaxFF simulations. After minimisation, which was performed for 95 conformers of $n$-dodecane using the reactive force field parameters, ${ }^{23}$ vibration frequencies were computed based on the mobile block Hessian (MBH) approach ${ }^{41}$ using the ADF optimiser. In $\mathrm{MBH}$, normal mode analysis was carried out using a fragmentation scheme, in which some parts of the Hessian matrix were diagonalised. These results allowed us to calculate the values of $G$ of $n$-dodecane conformers using the methods of statistical

mechanics. ${ }^{42}$ Figure S5 shows a nanodrop with 71 molecules used for assessment of PM7 and ReaxFF methods in comparison with AIMD results.

\section{Results and Discussions}

3-1 Electronic and bond energies and interatomic energy transfers. The long range corrected functional $\omega$ B97X-D allows us to obtain values of BE which are in good agreement with those obtained using the X1 (B3LYP with corrections for more accurate prediction of the bond energy) method (see Table 1a,b). The X1 method predicts bond dissociation energies in good accord with experimental data. ${ }^{43,44}$ For example, the C-C bond dissociation energy in the middle of an $n$-dodecane chain $\left(C_{6}-C_{7}\right)$ is estimated to be 84.3 and 86.6 $\mathrm{kcal} / \mathrm{mol}$ using $\mathrm{X} 1$ and $\omega \mathrm{B} 97 \mathrm{X}-\mathrm{D} / \mathrm{cc}-\mathrm{pVTZ}$ methods, respectively. These are in good agreement with the experimentally observed value of $85.0 \pm 2.0 \mathrm{kcal} / \mathrm{mol}{ }^{45}$ The comparison of results of calculations using $\omega \mathrm{B} 97 \mathrm{X}-\mathrm{D}$ and $\omega \mathrm{B} 97 \mathrm{X}$ functionals with the experimental results shows the importance of the dispersion force effects. These effects are dominant in the long chain hydrocarbons and are taken into account in the functional $\omega \mathrm{B} 97 \mathrm{X}-\mathrm{D}$, used in our analysis. If the dispersion corrections in the $\omega$ B97X-D functional are not taken into consideration the values of $\mathrm{BE}$ are underestimated by up to $7 \mathrm{kcal} / \mathrm{mol}$ depending on the type of chemical bonds (C-H or C-C) in the backbone of the $n$-dodecane. As follows from our analysis, the comparison of bond energies obtained by $\omega$ B97X-D/cc-pVTZ, semi-empirical 
PM7, and ReaxFF methods demonstrates that PM7 is less reliable for the analysis of $\mathrm{C}-\mathrm{H}$ and $\mathrm{C}-\mathrm{C}$ bond energies in an $n$-dodecane molecule than ReaxFF. The PM7 method underestimates the bond energies significantly (by approximately $25 \mathrm{kcal} / \mathrm{mol}$ ) in comparison with the $\omega \mathrm{B} 97 \mathrm{X}-\mathrm{D} / \mathrm{cc}-\mathrm{pVTZ}$ and X1 methods. The absolute errors associated with the application of the ReaxFF method were estimated to be in the region of $3.0 \mathrm{kcal} / \mathrm{mol}$. The values of $\mathrm{C}-\mathrm{H}$ and $\mathrm{C}-\mathrm{C}$ bond energies were over- and under-estimated, respectively, in comparison with the DFT results. Entropic effects significantly reduce the values of $G_{b o n d}^{\text {gas }}$ (see Table 1a,b) and the energy correction term (entropy effects) increases from 8-10 $\mathrm{kcal} / \mathrm{mol}$ for the $\mathrm{C}-\mathrm{H}$ bonds to $\sim 16 \mathrm{kcal} / \mathrm{mol}$ for the $\mathrm{C}-\mathrm{C}$ bonds. The bond energies calculated using the PM7, ReaxFF, wB97X and wB97X-D methods were compared with X1 results. The corresponding errors are shown in Figure 1. Note that the experimental bond energies are not available for all bonds of n-dodecane ${ }^{45}$. As can be seen in Figure 1, the accuracy of the ReaxFF method in determination of the bond energies $\mathrm{C}-\mathrm{H}$ and $\mathrm{C}-\mathrm{C}$ is better than that of PM7.

The observed vibrational (infrared) spectrum of $n$-dodecane and the vibrational normal modes computed using DFT, ReaxFF, and PM7 methods in the range of $0-4000 \mathrm{~cm}^{-1}$ are shown in Figure 2. Note that observational results are limited to the range $500-3700 \mathrm{~cm}^{-1}$. Our analysis predicts that the absorption in the ranges $0-500 \mathrm{~cm}^{-1}$ and $3700-4000 \mathrm{~cm}^{-1}$ is negligibly small. Both experimentally observed and computed spectra result from stretching, bending, scissoring, and rocking motions of the atoms in the $\mathrm{C}-\mathrm{C}$ and $\mathrm{C}-\mathrm{H}$ bonds. The vibrational dynamics in an $n$-dodecane molecule can play important roles in the evaporation/condensation processes, since they affect the intermolecular interactions in the liquid phase and the conformerisation of the molecules. A comparison of the predicted vibrational normal modes with the observed infrared spectrum shows that the DFT results are in much better agreement with experimental data than those obtained using the ReaxFF or 
PM7 methods. These spectra, as features describing the intermolecular dynamics, play an important role in the energy characteristics of molecules. Therefore, it is essential that the methods used in the analysis give a correct description of the vibrational spectra of molecules under consideration.

\section{3-2 Conformers, internal Gibbs free energies and intramolecular energy transfers.}

Entropic effects can play important roles in the evaporation process due to their enhancement in the gas phase, especially at high temperatures, and, therefore, their influence on the transfer of molecules from the liquid phase into the gas (vapour) phase. ${ }^{46}$ In the current study, the role of conformerisation effects in determining the Gibbs free energies of evaporation for n-dodecane conformers is investigated. The anharmonicity effects are studied a) using a set of conformers for $n$-dodecane molecules in both gas and liquid phases and b) based on the analysis of coupling for torsional and rotational dynamics in the gas phase. In the gas phase, the maximal $\Delta G_{\text {conf-rovib }}^{\text {gas } j}$ (changes in internal Gibbs free energy of various conformers relative to the base structure (structure 1) caused by conformational, rotational, torsional and vibrational dynamics) increases from $7 \mathrm{kcal} / \mathrm{mol}$ at $300 \mathrm{~K}$ to $10 \mathrm{kcal} / \mathrm{mol}$ at $650 \mathrm{~K}$ (see Figures 3a and S2a). The vibrational dynamics of bonds affects the torsional dynamics of functional groups in the conformers and the overall rotational dynamics and vice versa. This enhances the anharmonicity and conformerisation effects in molecules. The values of $\Delta G_{\text {conf-rovib }}^{\text {gas } j}$ increase with increasing temperature in different ways for different conformers and some conformers can be more stable than structure 1 (see Fig S2a). Figure 3b shows the probability of existence of various conformers at various temperatures in the gas phase. Comparing Figures 3a and 3b, one can see that the population of conformers with higher internal Gibbs free energies is smaller than that at lower $G_{\text {conf }- \text { rovib }}^{\text {gas } j}$. The mole fractions of conformers studied are in the range $0.00-0.15$ at $300 \mathrm{~K}$; this range becomes narrower at 
higher temperatures (see Fig. 3b). This means that almost all selected conformers can play important roles in the transfer of energy in the evaporation process at high temperatures.

The ratios of partition functions $\frac{Q_{\text {conf-rovib }}^{M S-T}}{Q_{\text {conf-rovib }}^{M S-H}}, \frac{Q_{\text {conf-rovib }}^{M S-H}}{Q_{\text {rovib }}^{S S-H}}$ and $\frac{Q_{\text {conf-rovib }}^{M S-T}}{Q_{\text {rovib }}^{S S-H}}$ versus temperature in the gas phase are shown in Figure 4. The first ratio (curve 'a') describes the effect of coupling between torsional and rotational dynamics (described by $\prod_{\tau=1}^{t} \varphi_{j, \tau}$ in Equation (1); see Section 'Computational methods'). This coupling is taken into account in partition function $Q_{\text {conf - rovib }}^{M S-T}$, but ignored in partition function $Q_{\text {conf - rovib }}^{M S-H}$. This ratio increases from 0.63 to 1.12 when temperature increases from $300 \mathrm{~K}$ to $650 \mathrm{~K}$. Ratio $\frac{Q_{\text {conf -rovib }}^{M S-H}}{Q_{\text {rovib }}^{S S-H}}$ (curve 'b') shows the relative importance of multi-structural effects without consideration of the anharmonicity coupling effects. As in the case of the first ratio, this ratio increases with temperature and its values are substantially larger than those for the first ratio. The third ratio $\frac{Q_{\text {conf -rovib }}^{M S-T}}{Q_{\text {rovib }}^{S S-H}}$ describes the role of multiple conformers alongside coupling effects due to anharmonicity. The rate of increase of this ratio with temperature is approximately the same as that of the second ratio. This demonstrates the reliability of the harmonic approximation in this range of temperatures in the gas phase. The fact that the second and third ratios are considerably larger than the first one suggests the importance of taking into account the contributions of various conformers in determination of the internal Gibbs free energy (see Figure S2a).

We expect that the second and third ratios would be even larger if a larger ensemble of conformers were studied $(N>95)$ due to entropic effects induced by conformerisation phenomena. For simplicity, only the variations in the values of the internal Gibbs free energy of some of the conformers are illustrated in Figure 3a (the values of $\Delta G_{\text {conf-rovib }}^{\text {gas } j}$ for all 95 
selected conformers are shown in Figure S2a). The average error of calculations of $\Delta G_{\text {conf-rovib }}^{\text {gas } j}$ for the conformers based on the harmonic approximation in the gas phase using PM7, in comparison with $\omega$ B97X-D/cc-pVTZ results, was $\sim 6 \mathrm{kcal} / \mathrm{mol}$. This error is half of the error resulting from the application of ReaxFF.

\section{3-3 Kinetic energies, molecular orientations and intermolecular energy transfers.}

In our previous paper ${ }^{32}$ it was shown that the kinetic energy of $n$-dodecane molecules can affect intermolecular interactions depending on the orientation of molecules hitting a droplet surface. Sticking or scattering of molecules to/from the surface of a nanodroplet depends strongly not only on the kinetic energy and the velocity of molecules but also on their orientations and collision angles. ${ }^{32}$ When an $n$-dodecane molecule was normal to a nanodroplet surface, the accommodation/scattering time was shorter in comparison with that in the case of a molecule moving parallel to the surface. This was attributed to the differences in multipoint interactions.

In order to study the influence of the internal molecular dynamics on the scattering/accommodation of molecules, two conformers of $n$-dodecane with the highest and average internal Gibbs free energies in the gas phase were selected (conformers 47 and 91). The molecular backbone plane and relevant velocity vectors were oriented normal and parallel to a nanodroplet surface (see Figure S5). As follows from our $a b$ initio MD results, these conformers have different accommodation times confirming the important role of the conformerisation effects of $n$-dodecane on scattering or accommodation at the interface.

The results of DFT, PM7 and ReaxFF calculations (see Table 2) show that in all cases PM7 overestimates the minimal value of the collision kinetic energy compared with DFT when scattering is expected. For a molecule orientated normal or parallel to the droplet surface, this value is greater than that predicted by DFT by 12 and $67 \mathrm{kcal} / \mathrm{mol}$, respectively. The errors of the results predicted by ReaxFF were $1-2 \mathrm{kcal} / \mathrm{mol}$ in these cases. These errors 
illustrate the importance of internal dynamics in long chain hydrocarbons such as $n$ dodecane. As PM7 does not include zero point energy (ZPE) effects and vibrational dynamics at lower than $100 \mathrm{~K}^{39}$, the conformerisation phenomena and conformational dynamics cannot be modelled properly using the PM7 method. On the other hand, in the ReaxFF parameterisation, ${ }^{22,23}$ the ZPE and conformerisation effects are taken into account properly using the corrections terms (see Equation $14 \mathrm{in}^{22}$ ). Therefore, the ReaxFF performed much better than PM7 in this study.

\section{3-4 Internal dynamics and entropy-enthalpy competition in the} evaporation/condensation processes. The internal dynamics of $n$-dodecane molecules (vibrational, rotational and conformational) can affect the Gibbs free energy of evaporation ( $\Delta G_{\text {conf-rovib }}^{e v}$ ), which is the difference between the internal Gibbs free energies of $n$-dodecane conformers in the gas $\left(G_{\text {conf-rovib }}^{\text {gas }}\right)$ and liquid $\left(G_{\text {conf-rovib }}^{\text {liq }}\right)$ phases. The contribution of the translational dynamics to the values of the Gibbs free energy of evaporation ( $\left.\Delta G^{e v}\right)$ was shown to be small for flexible molecules, including $n$-dodecane, in comparison with the contributions of their rotational, conformational and vibrational dynamics (see ${ }^{46,49}$ and SI). The internal Gibbs free energies of evaporation of each conformer $\left(\Delta G_{\text {conf-rovib }}^{e v, j}=G_{\text {conf-rovib }}^{\text {gas, }}-G_{\text {conf-rovib }}^{\text {liq, } j}\right.$, which are affected by the contributions of electronic polarisation (electrostatic components), cavitation, dispersion and structural reorganisation of the solvent (non-electrostatic components), increase with increasing temperature in most cases (see Figure 5a). The dependence of molar fractions of various conformers on temperature is shown in Figure 5b using the correction coefficient due to entropic effects, $\frac{\Delta T}{T}$, in the temperature range $300-650 \mathrm{~K} .^{50}$ The values of $\Delta G_{\text {conf-rovib }}^{e v, j}$ were shown to be up to +5.8 kcal/mol for some conformers and $-1.6 \mathrm{kcal} / \mathrm{mol}$ for the others at $650 \mathrm{~K}$ (see Figures 5a and S2b). The latter indicates higher stability of some folded conformers in the gas phase 
relative to the liquid phase (see structures 3, 9, 12, 15, 24 and 47 in Figure 6). Conformer 1 does not exist in the liquid phase since it corresponds to a second-order saddle point based on its force constant (frequency) calculation. The internal Gibbs free energy of evaporation of the ensemble of 94 conformers ( $\Delta G_{\text {conf-rovib }}^{e v}$ ), alongside the internal entropic $\left(-T \Delta S_{\text {conf-rovib }}^{e v}\right)$ and enthalpic ( $\Delta H_{\text {conf-rovib }}^{e v}$ ) contributions to the internal Gibbs free energy, calculated in both gas and liquid phases, and experimentally determined values of $\Delta H^{e v}$, are shown in Figure 7. As follows from this figure, the enthalpic contribution to the Gibbs free energy of evaporation decreases with temperature (from $13 \mathrm{kcal} / \mathrm{mol}$ to $2 \mathrm{kcal} / \mathrm{mol}$ when temperature is increased from $300 \mathrm{~K}$ to $650 \mathrm{~K}$ ), while the entropic contribution to this energy increases with temperature (from $-7 \mathrm{kcal} / \mathrm{mol}$ to $2 \mathrm{kcal} / \mathrm{mol}$ when temperature is increased from $300 \mathrm{~K}$ to $650 \mathrm{~K})$. The predicted values of $\Delta H_{\text {conf-rovib }}^{e v}$ turned out to be close to those observed experimentally. ${ }^{51}$ In order to validate the entropic effects induced by conformerisation of 95 conformers, the values of computed Gibbs free energies of evaporation were compared with experimental ones ${ }^{52}$ in the range of temperatures 300-650 K,. The experimental curve (see Fig 7) was obtained using the values of density of liquid $\left(\rho_{l}\right)$ and vapour $\left(\rho_{v}\right)$ at the vapourliquid saturation curve ${ }^{52}$ and the following expression: ${ }^{41}$

$$
\rho_{l}=\rho_{v} \exp \left(\frac{\Delta G_{s a t}^{e v}}{k_{B} T}\right)
$$

As can be seen in Fig. 7, our estimation of $\Delta G_{\text {conf-rovib }}^{e v}$ shows the same trend as the experimental results up to the boiling temperatures when a discontinuity was observed. Note that while the computed Gibbs free energies of evaporation have been calculated at constant pressure 1 atm, the experimental Gibbs free energies of evaporation in the saturation state, $\Delta G_{s a t}^{e v}$ in Equation (8), have taken into account the variations of the saturated pressures with temperatures ${ }^{52}$. 
At temperatures close to the boiling temperature at atmospheric pressure $(489 \mathrm{~K}) \mathrm{a}$ discontinuity in the temperature derivative of the Gibbs free energy of evaporation ( $\left.\Delta G_{\text {conf-rovib }}^{e v}\right)$ is observed. This is related to the corresponding discontinuity of the entropy contribution to this function. Note that no such discontinuity is observed for individual conformers (see Figures $5 \mathrm{a}$ and S2b). This difference in temperature dependence of $\Delta G_{\text {conf-rovib }}^{e v}$ for individual conformers and the ensemble of conformers is attributed to the fact that the mole fractions of the $n$-dodecane conformers change with temperature (see Figures 3b, 5b, S3 and S4). Note that the main contribution to $\Delta G_{\text {conf-rovib }}^{e v}$ for the ensemble of conformers comes from the conformers shown in Figure S2b rather than in Figure 5a (the values of $\Delta G_{\text {conf-rovib }}^{e v}$ for the ensemble at temperature $300 \mathrm{~K}$ are close to $6 \mathrm{kcal} / \mathrm{mol}$ ). The values of $\Delta G_{\text {conf-rovib }}^{e v}$ remain almost constant in the temperature range between the boiling temperature and close to the critical temperature. As all internal dynamics simulations in both phases have been performed based on finding relevant local minima referring to thermodynamic equilibrium states, our results can be applied to the modelling of not only evaporation, but also condensation processes.

\section{Conclusions}

The analysis of bond energies, Gibbs free energies of internal dynamics of a set of $n$ dodecane conformers, and collision energies of molecules hitting the surface of a nanodroplet shows that ReaxFF performs better than the semi-empirical PM7 method in terms of cost and accuracy of calculations. The reliability of ReaxFF for this study is confirmed based on the comparisons made between the predicted ReaxFF and PM7 results with the available experimental data, and the results of $a b$ initio $\mathrm{MD}$ and internal dynamics simulations using $\omega \mathrm{B} 97 \mathrm{X}-\mathrm{D} / \mathrm{cc}-\mathrm{pVTZ}$ electronic model chemistry. It is shown that the temperature dependence 
of the internal Gibbs free energy of evaporation of the ensemble of $n$-dodecane conformers differs considerably from the temperature dependence of this energy for individual conformers. This is attributed to the dependence of the molar fractions of conformers on temperature. Also, it is demonstrated, using the internal dynamics simulations, that the enthalpic and entropic contributions to the Gibbs free energy need to be taken into account in the analysis. Our findings demonstrate the importance of conformerisation phenomena in the energy transfers between gas and liquid phases during the evaporation/condensation processes in long chain molecules, such as $n$-dodecane.

\section{Figure Captions}

Figure 1. Error bar graph. The errors of the results predicted using PM7, ReaxFF, $\omega \mathrm{B} 97 \mathrm{XD}$ and $\omega \mathrm{B} 97 \mathrm{X}$ approaches relative to X1 results.

Figure 2. Infrared (IR) absorbance spectra of $\boldsymbol{n}$-dodecane. IR absorption at 3100-2800 $\mathrm{cm}^{-1}$ is related to asymmetric and symmetric C-H stretching vibrations. The $\mathrm{H}-\mathrm{C}-\mathrm{H}$ bending (scissoring) vibrations correspond to bands at $1465-1370 \mathrm{~cm}^{-1}$. The C-C stretching vibrations are responsible for the part of the spectrum between $1300 \mathrm{~cm}^{-1}$ and $850 \mathrm{~cm}^{-1}$. The experimental data were collected from the NIST/EPA Gas-Phase Infrared Database. The DFT, ReaxFF and PM7 calculations were performed using the ADF optimiser (see Section 2).

Figure 3. The internal Gibbs free energies and mole fractions of selected $\boldsymbol{n}$-dodecane conformers in the gas phase. a, the internal Gibbs free energy was calculated relative to the most stable conformers (see structure 1) at 300-650 K and 1 atm (conf. and rovib. refer to conformational and rotational/vibration effects, respectively). Optimisation and frequency calculations have been performed using the $\omega$ B97X-D/cc-pVTZ chemistry models and MS-H approximation (see Figure S2a for all selected conformers). b, mole fractions of selected conformers of $n$-dodecane computed using $\omega$ B97X-D/cc-pVTZ and MS-H models in the gas phase (see Figure S3 for all conformers). The structures of the conformers used in this figure are shown in Figure 5.

Figure 4. The ratios of partition functions for $n$-dodecane conformer(s) versus temperature: $\frac{Q_{\text {conf - rovib }}^{M S-T}}{Q_{\text {conf - rovib }}^{M S-H}}$ (a), $\frac{Q_{\text {conf - rovib }}^{M S-T}}{Q_{\text {rovib }}^{S S-H}}$ (b), $\frac{Q_{\text {conf -rovib }}^{M S-H}}{Q_{\text {rovib }}^{S S-H}}$ (c). The calculations were based on 95 conformers of an $n$-dodecane molecule and the $\omega$ B97X-D/cc-pVTZ model.

Figure 5. Internal molecular dynamics effects in the evaporation/condensation processes and mole fractions of selected $\boldsymbol{n}$-dodecane conformers in the liquid phase. a, the internal Gibbs free energies of evaporation $\left(\Delta G_{\text {conf-rovib }}^{\text {ev,j }}=G_{\text {conf-rovib }}^{\text {gas }, j}-G_{\text {conf }- \text { rovib }}^{\text {liq }, j}\right.$ ) for selected $n$ - 
dodecane conformers (for all conformers see Figure S2b) between gas and liquid phases with temperatures obtained using $\omega B$ 97X-D/cc-pVTZ, SMD- $\omega B 97 X-D / c c-p V T Z$ and MS-H models. $\mathbf{b}$, mole fractions of selected conformers of $n$-dodecane computed using the SMD$\omega B 97 X-D / c c-p V T Z$ method and MS-H models in the liquid phase (Figure S4 shows the contributions of 94 conformers under consideration in the liquid phase).

Figure 6. The structures of the conformers used in Figure 2.

Figure 7. Internal entropy-enthalpy competition. The temperature dependence of the internal Gibbs free energy of evaporation $\left(\Delta G_{\text {conf-rovib }}^{e v}\right.$ ) of an ensemble of $94 n$-dodecane conformers and its enthalpic ( $\Delta H_{\text {conf-rovib }}^{e v}$ ) and entropic $\left(-T \Delta S_{\text {conf-rovib }}^{e v}\right.$ ) contributions, calculated using a combination of $\omega$ B97X-D/cc-pVTZ, SMD- $\omega$ B97X-D/cc-pVTZ and MS-H methods. The experimental curves, inferred from ${ }^{51,52}$, are also shown. 
Table 1. $\mathrm{C}_{12} \mathrm{H}_{26}$ bond energies (BE) and bond contribution to Gibbs free energies ( $G_{\text {bond }}^{\text {gas }}$ ) of $\boldsymbol{n}$-dodecane (see below) predicted by various methods.

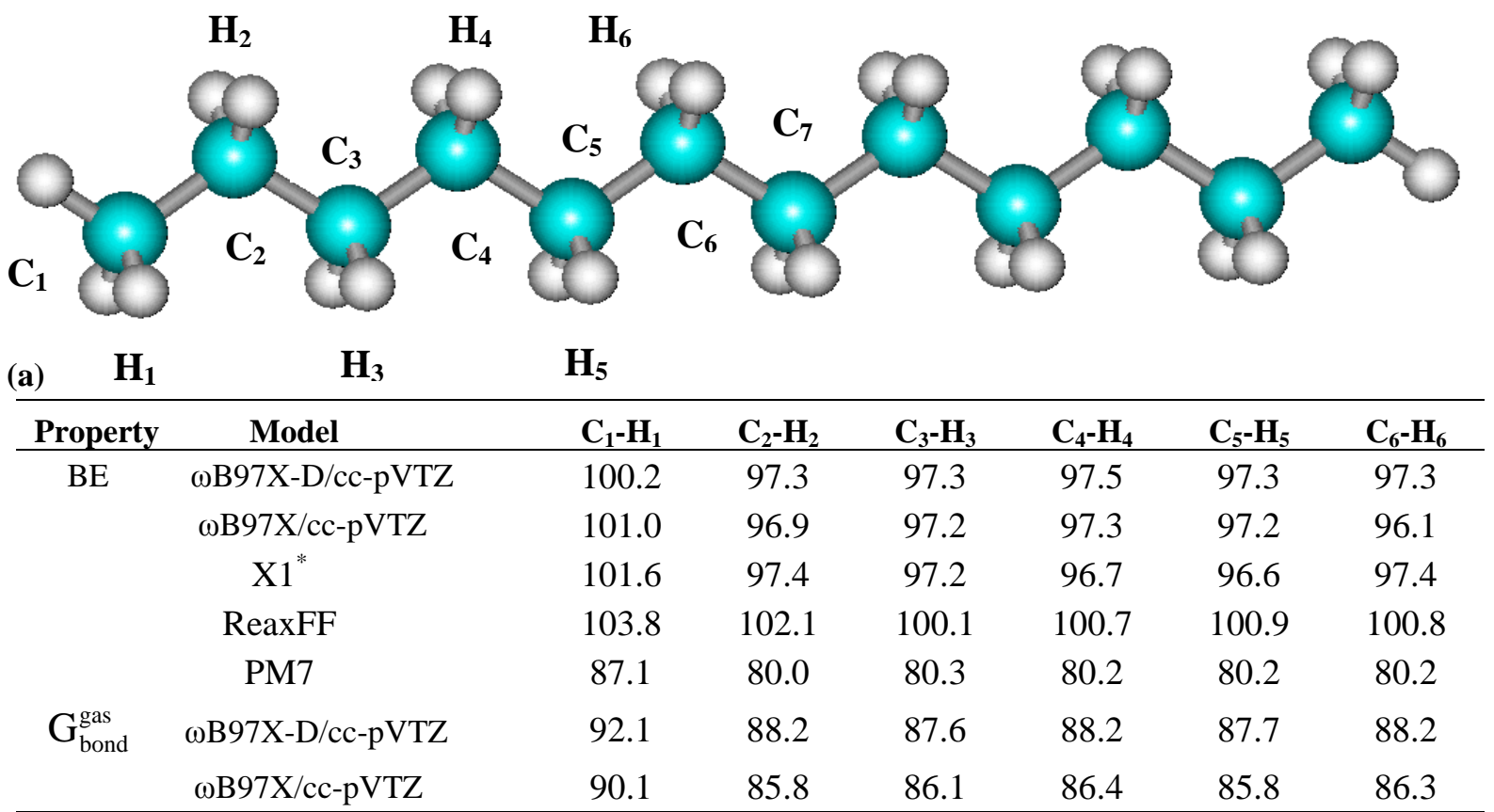

(b)

\begin{tabular}{cccccccc}
\hline Property & Model & $\mathbf{C}_{1}-\mathbf{C}_{2}$ & $\mathbf{C}_{2}-\mathbf{C}_{3}$ & $\mathbf{C}_{3}-\mathbf{C}_{4}$ & $\mathbf{C}_{4}-\mathbf{C}_{5}$ & $\mathbf{C}_{5}-\mathbf{C}_{6}$ & $\mathbf{C}_{6}-\mathbf{C}_{7}$ \\
\hline $\mathrm{BE}$ & $\omega \mathrm{B} 97 X-\mathrm{D} / \mathrm{cc}-\mathrm{pVTZ}$ & 88.5 & 86.4 & 88.0 & 87.2 & 88.0 & 86.6 \\
& $\omega \mathrm{B} 97 \mathrm{X} / \mathrm{cc}-\mathrm{pVTZ}$ & 89.8 & 87.3 & 88.2 & 80.1 & 80.8 & 79.5 \\
& $\mathrm{X1}^{*}$ & 88.9 & 86.0 & 85.6 & 85.4 & 84.6 & 84.3 \\
& $\mathrm{ReaxFF}$ & 86.9 & 81.4 & 81.5 & 81.8 & 81.7 & 81.8 \\
& $\mathrm{PM7}$ & 68.0 & 61.0 & 62.2 & 62.1 & 62.1 & 62.1 \\
$\mathrm{G}_{\text {bond }}^{\text {gas }}$ & $\omega \mathrm{B} 97 \mathrm{X}-\mathrm{D} / \mathrm{cc}-\mathrm{pVTZ}$ & 75.9 & 72.1 & 72.7 & 71.4 & 72.7 & 72.0 \\
& $\omega \mathrm{B} 97 \mathrm{X} / \mathrm{cc}-\mathrm{pVTZ}$ & 75.0 & 71.6 & 72.1 & 71.4 & 72.0 & 71.8 \\
\hline
\end{tabular}

a, values of $\mathrm{BE}$ and $\mathrm{G}_{\text {bond }}^{\text {gas }}$ for C-H bonds (in kcal/mol), obtained using three DFT methods ( $\omega$ B97X-D/ccpVTZ, $\omega$ B97X/cc-pVTZ and X1 (B3LYP with corrections)), ReaxFF and PM7 methods; $\mathbf{b}$, the same values, but for C-C bonds; * indicates data taken from ${ }^{25}$.

Table 2. Critical initial kinetic energies at a large distance from the cluster* (in $\mathrm{kcal} / \mathrm{mol}$ ) required for the reflection of oncoming molecules.

\begin{tabular}{ccccccc} 
& \multicolumn{2}{c}{ Conformer 1 } & \multicolumn{2}{c}{ Conformer 47 } & \multicolumn{2}{c}{ Conformer 91 } \\
\cline { 2 - 6 } Method & $\begin{array}{c}\text { Normal to } \\
\text { surface }\end{array}$ & $\begin{array}{c}\text { Parallel } \\
\text { to surface }\end{array}$ & $\begin{array}{c}\text { Normal } \\
\text { to surface }\end{array}$ & $\begin{array}{c}\text { Parallel to } \\
\text { surface }\end{array}$ & $\begin{array}{c}\text { Normal to } \\
\text { surface }\end{array}$ & $\begin{array}{c}\text { Parallel to } \\
\text { surface }\end{array}$ \\
\hline DFT & 73.8 & 128.8 & 66.7 & 119.6 & 62.4 & 113.1 \\
PM7 & 85.4 & 195.6 & 78.4 & 188.9 & 78.5 & 181.4 \\
ReaxFF & 75.1 & 131.2 & 68.2 & 118.5 & 61.3 & 114.7 \\
\hline
\end{tabular}

* The effects of collisions of individual molecules with the surface of a nanodroplet with $70 n$-dodecane molecules were studied using the DFT, PM7 and ReaxFF methods (see Figure S5). 


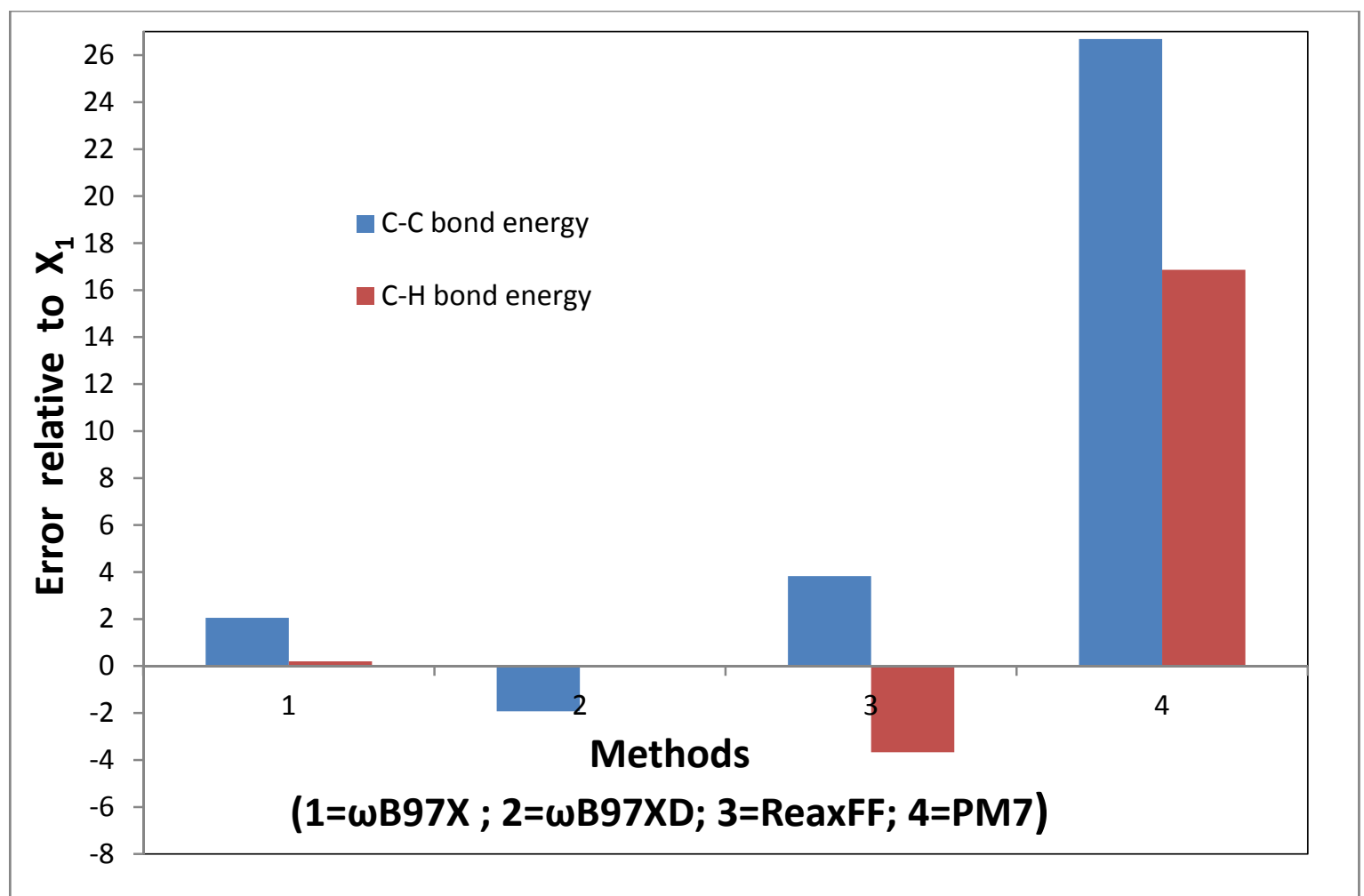

Figure 1

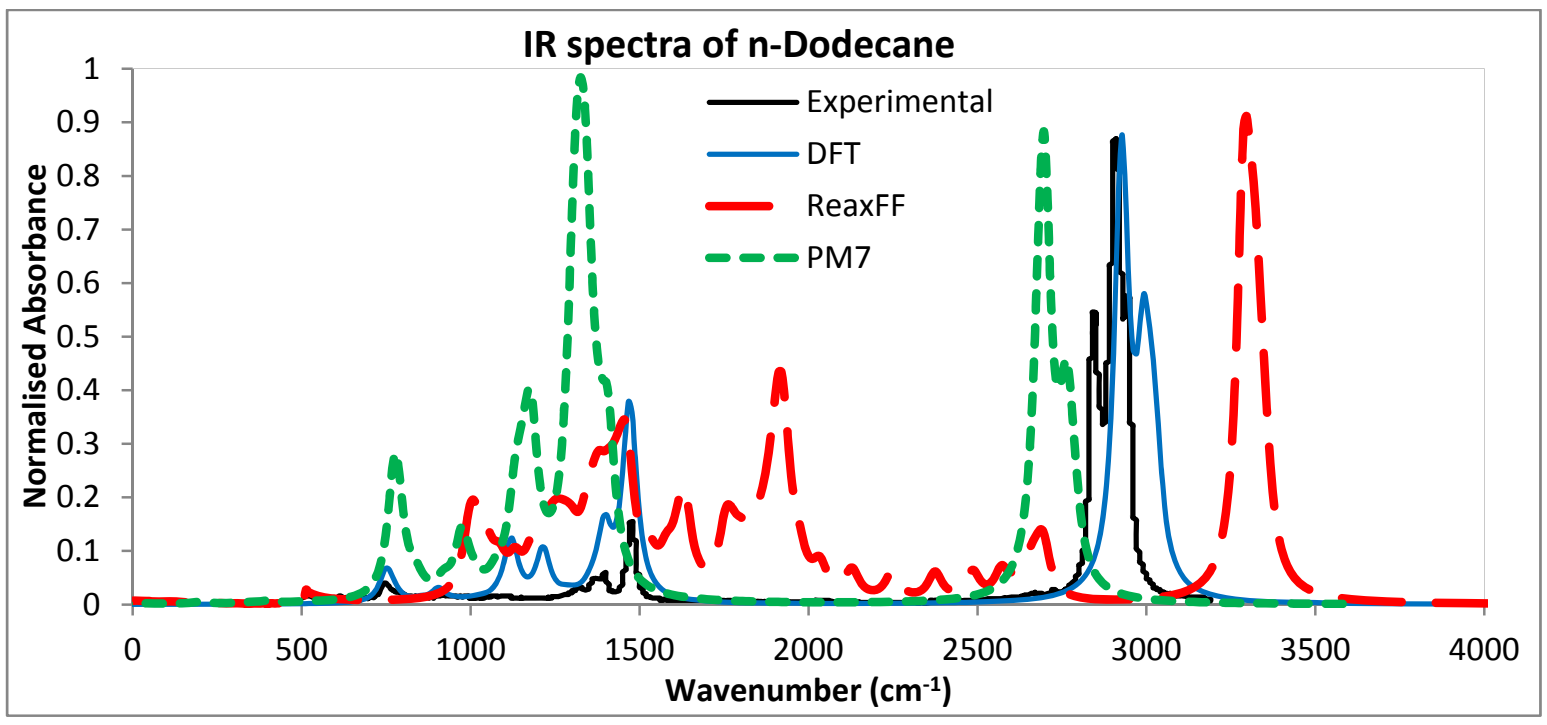

Figure 2 

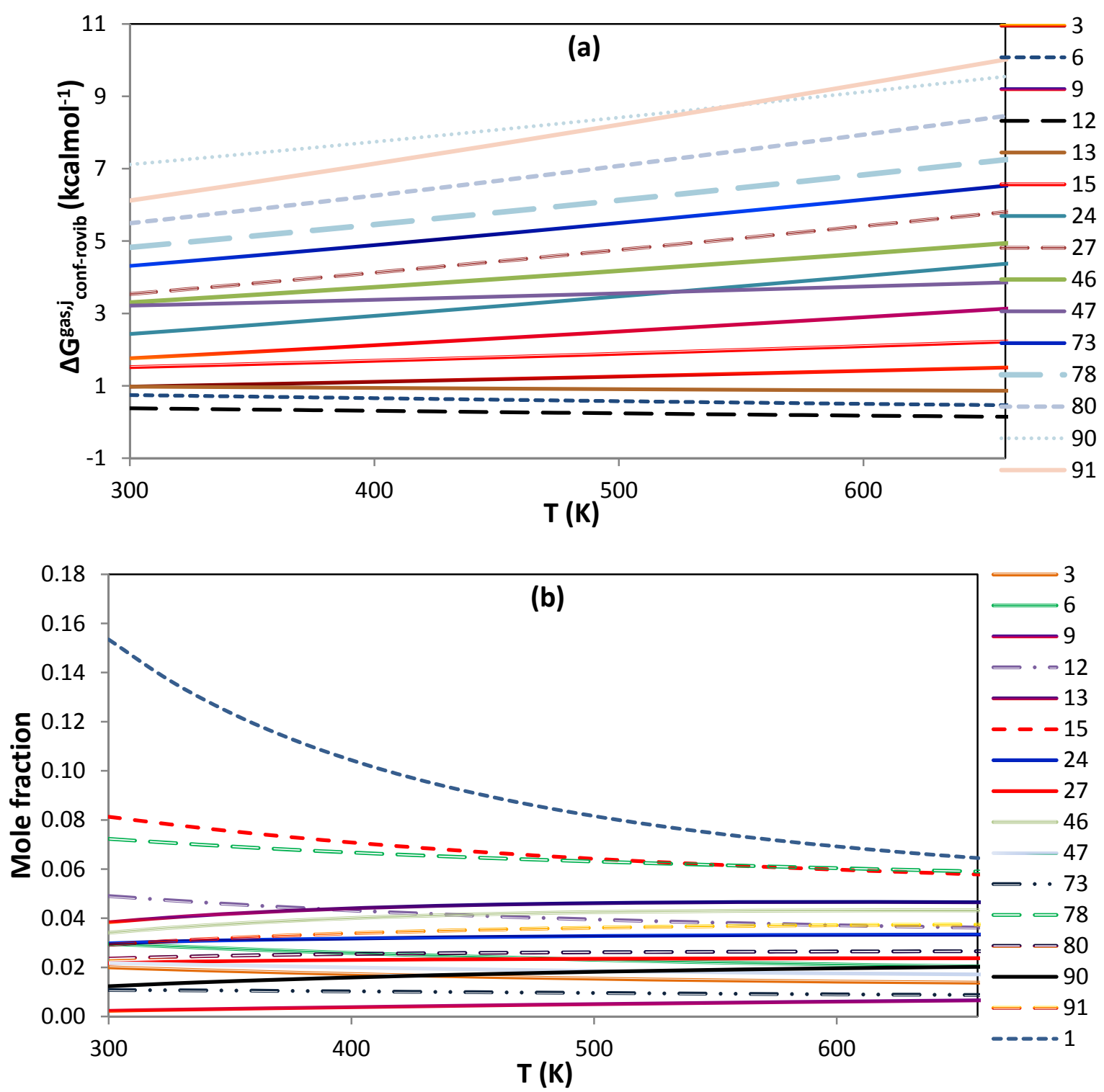

Figure 3 


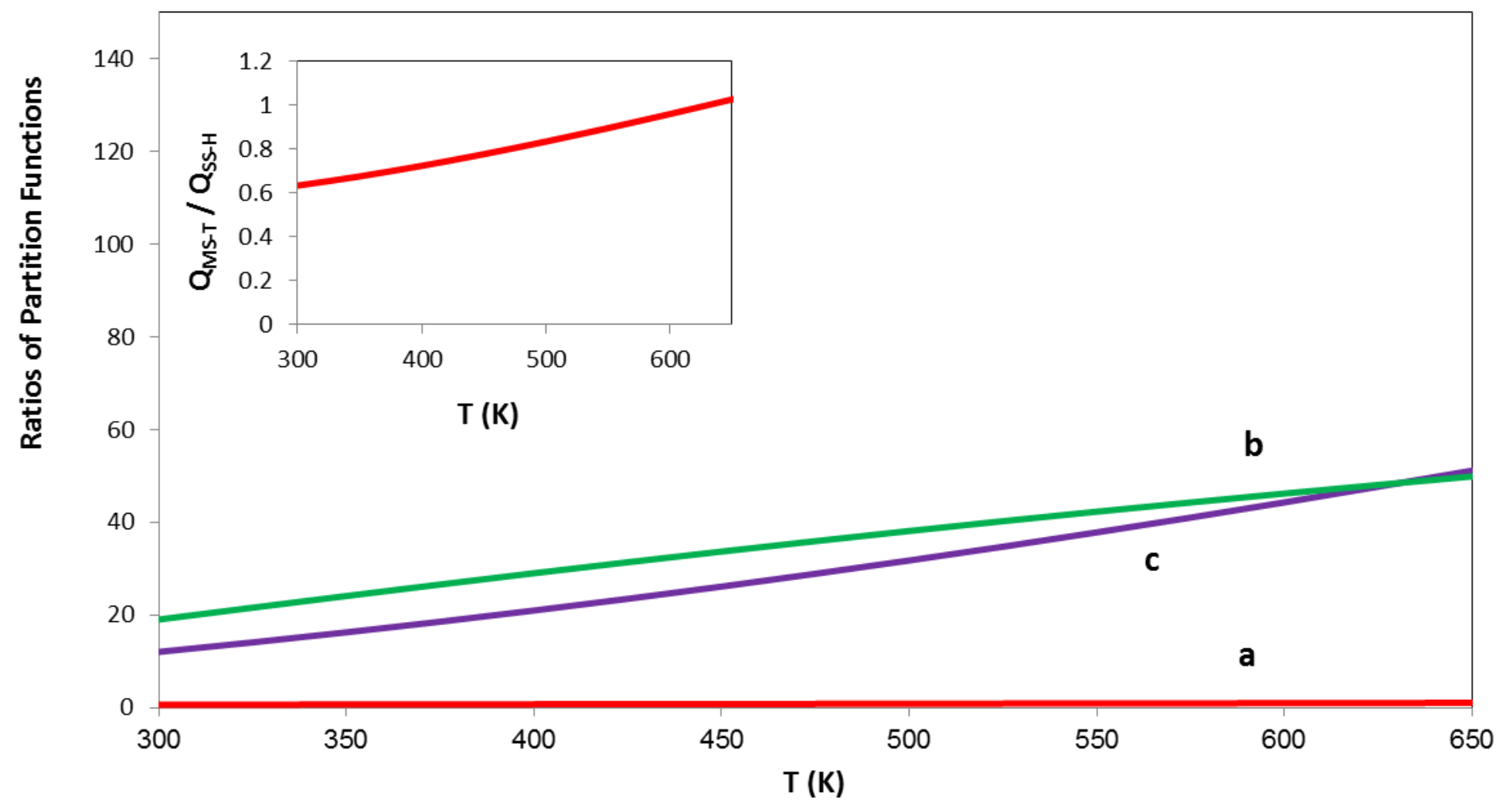

Figure 4 

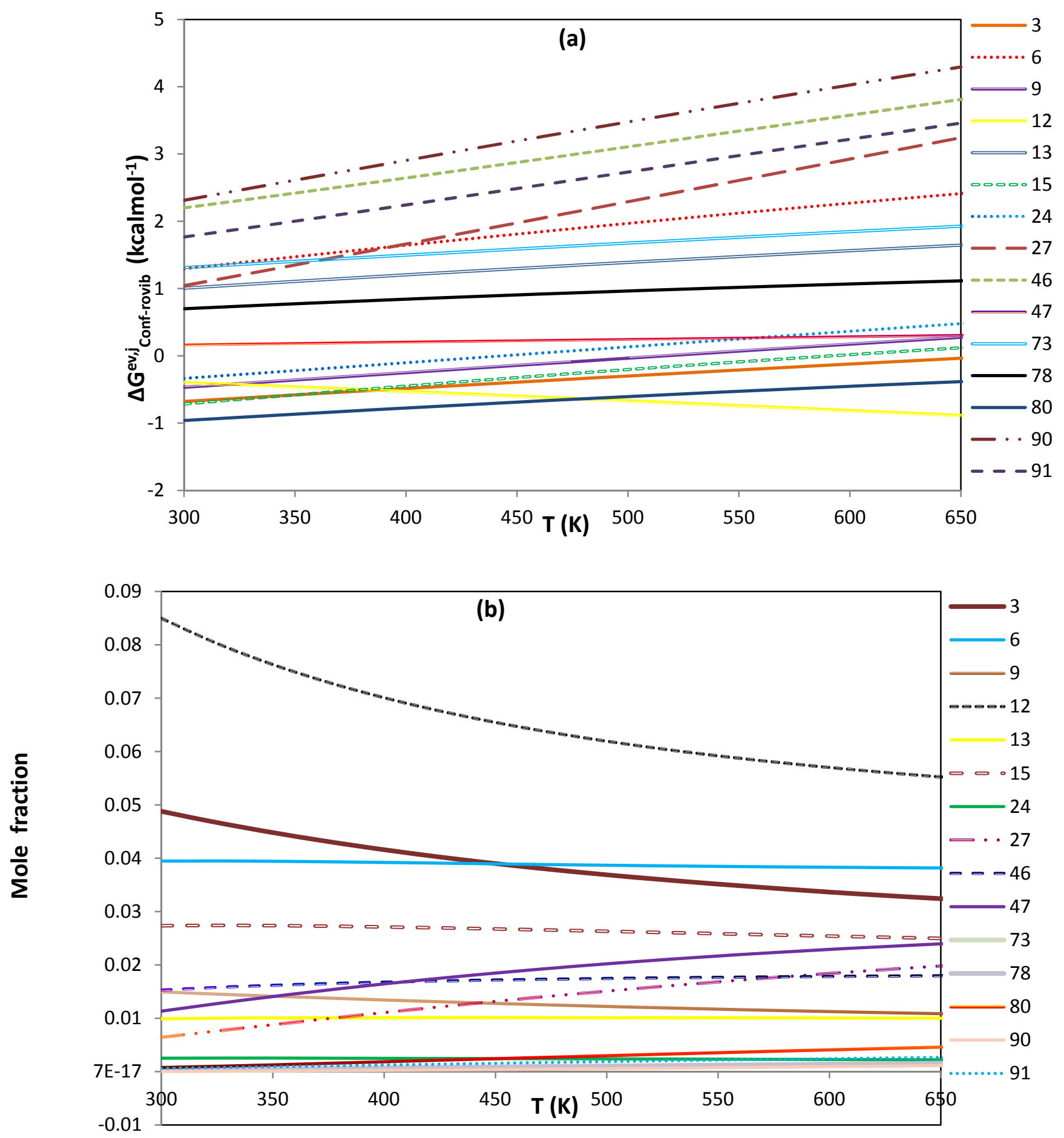

Figure 5 


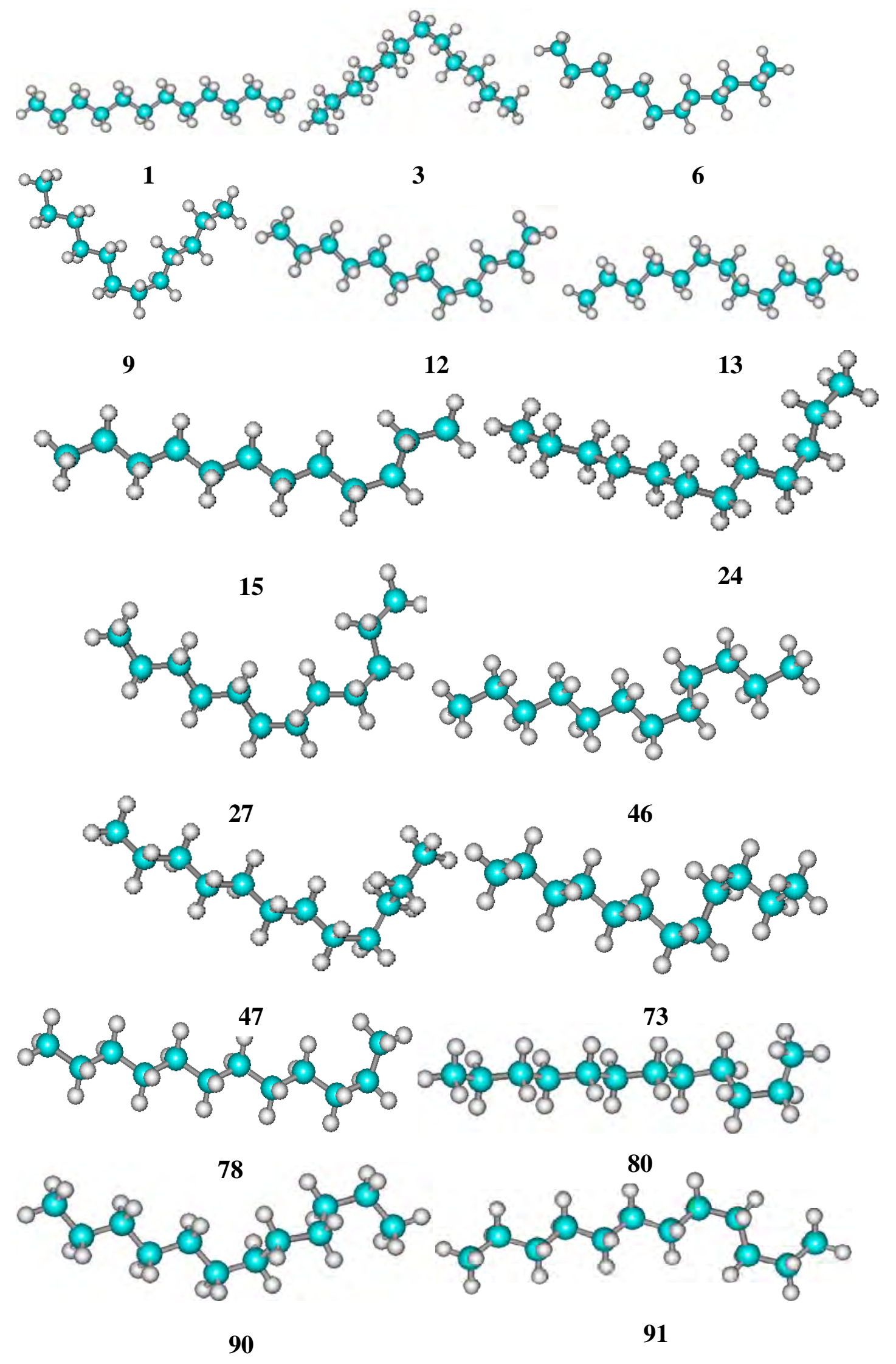

Figure 6 


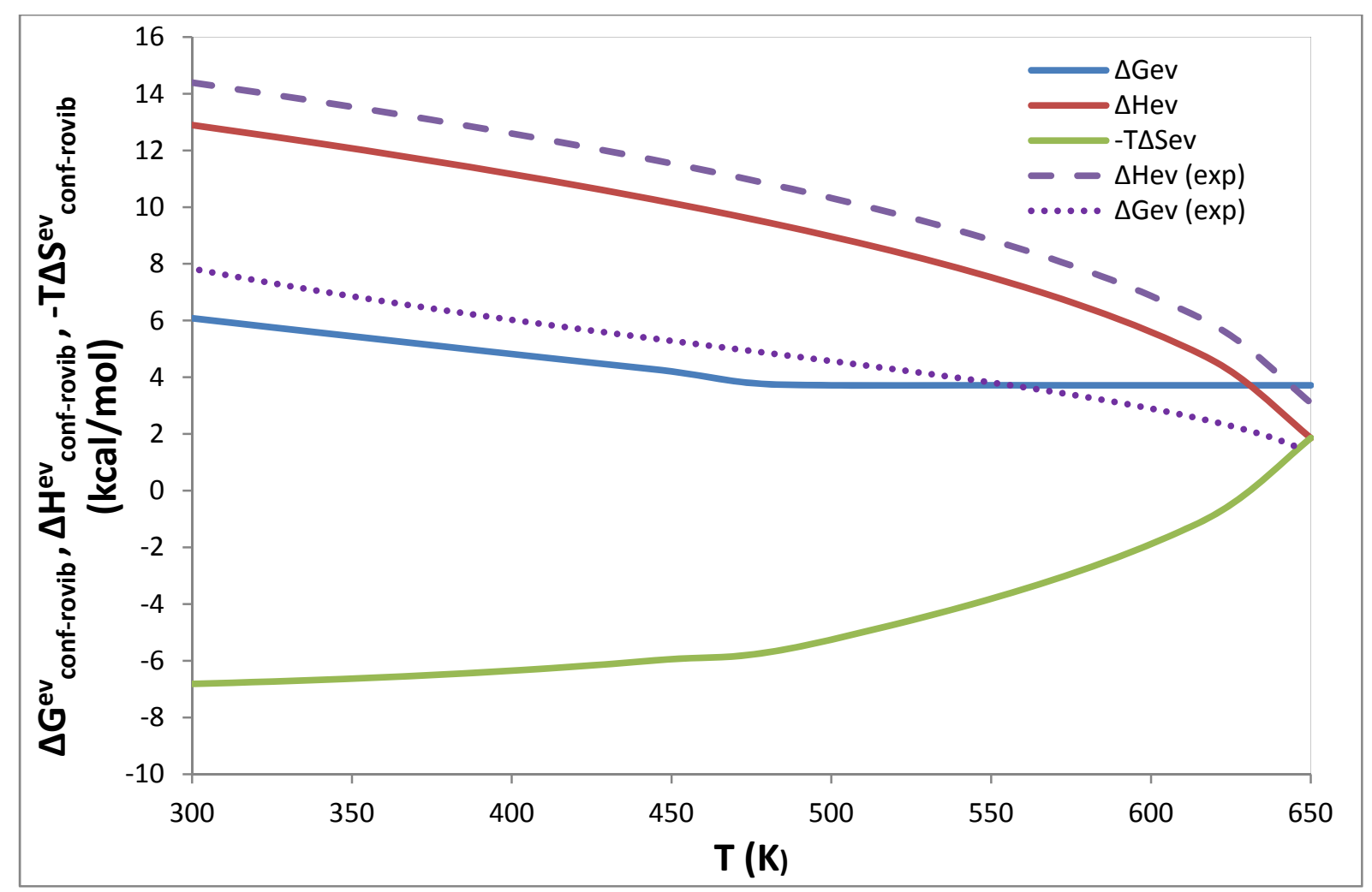

Figure 7

\section{Supporting information}

The authors declare no competing financial interests. Supplementary information includes text for internal molecular dynamics (IMD) effects; $\omega$ B97X-D/cc-pVTZ optimised Cartesian coordinates for $95 n$-dodecane conformers investigated in this study; the relative energies for all of these structures; mole fractions of $n$-dodecane conformers in the gas and liquid phases at temperatures in the range 300-650 $\mathrm{K}$ calculated using the $\omega \mathrm{B} 97 \mathrm{X}-\mathrm{D} / \mathrm{cc}-\mathrm{pVTZ}$, SMD$\omega B 97 X-D / c c-p V T Z$ and MS-H models; schematic nanodrop studied for assessment of PM7 and ReaxFF methods using AIMD calculations and zero-point energies of all molecules.

\section{Acknowledgements}

The authors are grateful to Professor Truhlar's group to provide us the MSTor program, Professor Martin J. Field (University of Grenoble, IBS) for useful discussions and the EPSRC (UK) (grant EP/J006793/1) for their financial support of this project. The use of NSCCS (http://www.nsccs.ac.uk/) and HECToR/ARCHER (http://www.archer.ac.uk/) supercomputers is gratefully acknowledged. 


\section{References}

1 Valero, F. P. J. Collins, W. D. Pilewskie, P. Bucholtz, A. Flatau, P. J. Direct radiometric observations of the water vapor greenhouse effect over the equatorial pacific ocean. Science 275, 1773-6 (1997).

2 Wheeler, T. D. Stroock, A. D. The transpiration of water at negative pressures in a synthetic tree. Nature 455, 208-12 (2008).

3 Sazhin, S. S. Advanced models of fuel droplet heating and evaporation. Prog. Energy Combust. Sci. 32, 162-214 (2006).

4 Fujikawa, S. Yano, T. Watanabe, M. Vapor-liquid interfaces, bubbles and droplets. Springer-Verlag, Heidelberg (2011).

5 Sone, Y. Kinetic theory and fluid dynamics. Birkh"auser, Boston (2002).

6 Ishiyama, T. Fujikawa, S. Kurz, T. Lauterborn, W. Non-equilibrium kinetic boundary condition at the vapor-liquid interface of argon. Phys. Rev. E. 88, 042406 (2013).

7 Miles, R. E. H. Knox, K. J. Reid, J. P. Laurain, A. M. C. Mitchem, L. Measurements of mass and heat transfer at a liquid water surface during condensation or evaporation of a subnanometer thickness layer of water. Phys. Rev. Lett. 105, 116101 (2010).

8 Varilly, P. Chandler, D. Water evaporation: a transition path sampling study. J. Phys. Chem. B 117, 1419 (2013).

9 Musolino, N. Trout, B. L. Insight into the molecular mechanism of water evaporation via the finite temperature string method. J. Chem. Phys. 138, 134707 (2013).

10 T. Ishiyama, T. Yano, T. Fujikawa, S. Molecular dynamics study of kinetic boundary condition at an interface between argon vapor and its condensed phase. Phys. Fluids 16, 4713 (2004).

11 Smith, J. D. Cappa, C. D. Drisdell, W. S. Cohen, R. C. Saykally, R. J. Raman thermometry measurements of free evaporation from liquid water droplets. J. Am. Chem. Soc. 128, 12892-12898 (2006).

12 Hickman K. C. D. Maximum evaporation coefficient of water. Indust. Eng. Chem. 46, 1442-1446 (1954).

13 Xia, T. K. Landman, U. Molecular evaporation and condensation of liquid $n$-alkane films. J. Chem. Phys. 101, 2498-2507 (1994).

14 Cao, B.-Y. Xie, J.-F. Sazhin, S.S. Molecular dynamics study on evaporation and condensation of $n$-dodecane at liquid-vapor phase equilibria. J. Chem. Phys. 134, 164309 (2011).

15 Xie, J.-F. Sazhin, S.S. Cao, B.-Y. Molecular dynamics study of the processes in the vicinity of the $n$-dodecane vapour/liquid interface. Phys. Fluids 23, 112104 (2011).

16 Holyst, R. et. al. Evaporation of freely suspended single droplets: experimental, theoretical and computational simulations. Rep. Prog. Phys. 76, 034601 (2013).

17 Winkler, P.M. et al. Mass and thermal accommodation during gas-liquid condensation of water. Phys.. Rev. Lett. 93, 075701 (2004).

$18 \mathrm{Li}, \mathrm{Y}$. Q. et al. Mass and thermal accommodation coefficients of H2O (g) on liquid water as a function of temperature. J. Phys. Chem. A 105, 10627-10634 (2001).

19 Gun'ko, V. M. Turov, V.V. Nuclear magnetic resonance studies of interfacial phenomena; surfactant science series volume 154, (New York: CRC Press, Taylor \& Francis Group 2013).

20 Goodman, F. O. Wachman, H. Y. In Dynamics of gas-surface scattering (Academic Press: New York 1976).

21 Echeverría, J. Aullón, G. Danovich, D. Shaik, S. Alvarez, S. Dihydrogen contacts in alkanes are subtle but not faint. Nat. Chem. 3, 323-330 (2011). 
22 Van Duin, A. C. T. Dasgupta, S. Lorant, F. Goddard, W. A., III. ReaxFF: A reactive force field for hydrocarbons. J. Phys. Chem. A 105, 9396 (2001).

23 Chenoweth, K. van Duin, A. C. T. Goddard, W. A., III. ReaxFF reactive force field for molecular dynamics simulations of hydrocarbon oxidation. J. Phys. Chem. A 112, 1040 (2008).

24 Ding, J. Zhang, L. Zhang, Y. Han, K-L. A reactive molecular dynamics study of $n$ heptane pyrolysis at high temperature. J. Phys. Chem. A 117, 3266-3278 (2013).

25 Wang, Q.-D. Wang, J.-B. Li, J.-Q. Tan, N.-X. Li, X.-Y. Reactive molecular dynamics simulation and chemical kinetic modelling of pyrolysis and combustion of $n$-dodecane. Combust. Flame 158, 217-226 (2011).

26 Cheng, X.-M. Wang, Q.-D. Li, J.-Q. Wang, J.-B. Li, X.-Y. ReaxFF molecular dynamics simulations of oxidation of toluene at high temperatures. J. Phys. Chem. A 116, 9811-9818 (2012).

27 Bagri, A. et. al. Structural evolution during the reduction of chemically derived graphene oxide. Nat. Chem. 2, 581-587 (2010).

28 Chenoweth, K. van Duin, A. C. T. Dasgupta, S. Goddard, W. A., III. Initiation mechanisms and kinetics of pyrolysis and combustion of JP-10 hydrocarbon jet fuel. $J$. Phys. Chem. A 113, 1740-1746 (2009).

29 Nasiri, R. Gun'ko, V. M.. Sazhin, S. S. Quantum Mechanical Effects in n-alkane Droplets. ILASS - Europe, 25th European Conference on Liquid Atomization and Spray Systems, Chania, Greece, 1-4 September (2013).

30 Chai, J. D. Head-Gordon, M. Long-range corrected hybrid density functionals with damped atom-atom dispersion corrections. Phys. Chem. Chem. Phys. 10, 6615-20 (2008).

31 Stewart, J. J. P. Optimization of parameters for semi-empirical methods VI: more modifications to the NDDO approximations and re-optimization of parameters. J. Mol. Model. 19, 1-32 (2013).

32 Gun'ko, V. M. Nasiri, R. Sazhin, S. S. A study of the evaporation and condensation of n-alkane clusters and nanodroplets using quantum chemical methods. Fluid Phase Equilibria 366, 99-107 (2014).

33 Martin, J. M. L. What can we learn about dispersion from the conformer surface of npentane? J. Phys. Chem. A 117, 3118-32 (2013).

34 Zheng, J. Mielke, S. L, Clarkson, K. L. Truhlar, D. G. MSTor: A program for calculating partition functions, free energies, enthalpies, entropies, and heat capacities of complex molecules including torsional anharmonicity. Comp. Phys. Comm. 183, 1803-1812 (2012).

35 Frisch, M. J. et al. Gaussian 09, revision D.01; Gaussian, Inc.: Wallingford, CT (2009).

36 VandeVondele, J. Krack, M. Mohamed, F. Parrinello, M. Chassaing, T. Hutter, J. Quickstep: Fast and accurate density functional calculations using a mixed Gaussian and plane waves approach. Comp. Phys. Comm. 167, 103 (2005).

37 Guidon, M.; Hutter, J.; VandeVondele, J. Auxiliary density matrix methods for Hartree-Fock exchange calculations. J. Chem. Theory Comput. 6, 2348-2364 (2010).

38 Goedecker, S.; Teter, M.; Hutter, J. Separable dual-space Gaussian pseudopotentials. Phys. Rev. B. Condens Matter. 54, 1703-1710 (1996).

39 Stewart, J. J. P. MOPAC 2012, Versions 13.123W and 13.123L, Stewart Computational Chemistry, Colorado Springs, CO, USA (2013).

40 Velde, G. te et al. Chemistry with ADF. J. Comp. Chem. 22, 931 (2001).

41 Ghysels, A. et al. Vibrational modes in partially optimized molecular systems. $J$. Chem. Phys. 126, 224102 (2007).

42 McQuarrie, D. A. Statistical Mechanics (Happer \& Row: New York, 1973). 
$43 \mathrm{Wu}, \mathrm{J} . \mathrm{M} . \mathrm{Xu}, \mathrm{X}$. Improving the B3LYP bond energies by using the $X 1$ method. J. Chem. Phys. 127, 214105 (2007).

44 Zhang, I.Y. Wu, J.M. Xu, X. Extending the reliability and applicability of B3LYP Chem. Commun. 46, 3057-3070 (2010).

45 Y.R. Luo. Handbook of Bond Dissociation Energies in Organic Compounds, CRC Press (2007).

46 Ben-Naim, A. Molecular theory of solutions; Oxford University Press Inc., New York (2006).

47 Marenich, A.V. Cramer, C.J.; Truhlar, D. G. Universal solvation model based on solute electron density and on a continuum model of the solvent defined by the bulk dielectric constant and atomic surface tensions. J. Phys. Chem. B, 113, 6378-6396 (2009).

48 Winget, P. Dolney, D.M. Giesen, D.J. Cramer, C.J. Truhlar, D.G. Minnesota Solvent Descriptor Database, University of Minnesota, 2010.

49 Ribeiro, R. F. Marenich, A. V. Cramer, C. J. Truhlar, D. G. Use of solution-phase vibrational frequencies in continuum models for the free energy of solvation. J. Phys. Chem. B 115, 14556-14562 (2011).

50 Pham, H. H. Taylor, C. D. Henson, N. J. First-principles prediction of the effects of temperature and solvent selection on the dimerization of benzoic acid. J. Phys. Chem. B 117, 868-876 (2013).

51 Thermophysical Properties of Chemicals and Hydrocarbons, edited by C. L. Yaws (William Andrew Inc.,Norwich, NY, 2008).

52 NIST Chemistry WebBook, saturation properties for n-Dodecane-temperature increments. http://webbook.nist.gov/chemistry/[accesed 17.01.15]. 


\title{
The Effects of Conformational Molecular Dynamics on the Evaporation/Condensation of $\boldsymbol{n}$-Dodecane
}

\author{
Rasoul Nasiri ${ }^{1}$, Vladimir M. Gun’ko ${ }^{1,2}$, Sergei S. Sazhin ${ }^{1_{*}}$
}

${ }^{1}$ Sir Harry Ricardo Laboratories, Centre for Automotive Engineering, School of Computing, Engineering and Mathematics, Faculty of Science and Engineering, University of Brighton, Brighton BN2 4GJ, UK

${ }^{2}$ Chuiko Institute of Surface Chemistry, 17 General Naumov Street, Kiev 03164, Ukraine

\section{Supplementary Information}

* Corresponding author. Tel.: +44 127364 2677; fax: +44 127364233

E-mail address: S.Sazhin@brighton.ac.uk 


\section{Table of contents}

Subject

Effects of internal molecular dynamics (IMD)

Figure S1: The five-step process of evaporation

Figure S2a: Relative Gibbs free energy of $95 n$-dodecane conformers

Figure S2b: Difference in internal Gibbs free energy

Figure S3: Mole fractions of conformers in the gas phase

Figure S4: Mole fractions of $n$-dodecane molecules in the liquid phase

Figure S5: Structures of a molecule attacking a nanodroplet

Zero point energy of the conformers

S7-S8

Optimised Cartesian coordinates, relative electronic energies, symmetry points and moments of inertia

References

S92 


\section{Effects of internal molecular dynamics (IMD) on evaporation of n-dodecane}

We assume that liquid and gas phases are in equilibrium at the same temperature and pressure. In order to show how IMD affect the Gibbs free energy of evaporation of $n$ dodecane molecules, the $N, P, T$ (number of conformers, pressure and temperature) ensemble is applied to predict the Gibbs free energy of evaporation $\left(\Delta G_{\mathrm{ev}}\right)$ using molecular solvation theory. An $n$-dodecane molecule $\left(\mathrm{C}_{12}\right)$ is transferred from a fixed position with respect to the centre of mass (COM) in the liquid phase into a fixed position in the ideal gas phase (see Figure S1). In the conventional modelling of evaporation, one needs to specify standard states, in both gaseous and liquid phases, for the calculation of the Gibbs free energy of evaporation: ${ }^{1,2}$

$$
\Delta G(l \rightarrow g)=G^{g}-G^{l}=\Delta G^{e v} .
$$

In contrast to the conventional approach, ${ }^{3}$ we will not specify any standard states for the evaporation of $\mathrm{C}_{12}$. Translational modes in the gas and liquid phases do not have significant effects in $\Delta G(l \rightarrow g)$, which allows us to simplify Eq. (S1) to $\Delta G^{e v}=\Delta G_{\text {conf-rovib }}^{e v}=G_{\text {confr-rovib }}^{\text {gas }}-G_{\text {conf-rovib }}^{\text {liq }}$, where subscript 'conf-rovib' refers to conformational, rotational and vibrational dynamics. These include both the IMD and coupling work (contributions of electronic-polarisation (EP) and cavitation, dispersion and solvent structure (CDS)) of $\mathrm{C}_{12}$ molecules in the liquid phase. $\mathrm{C}_{12}$ molecules (in our study, 95 conformers) show a multi-structural behaviour and each of them has a different binding energy in the liquid (see Table 2 in the main text). ${ }^{3}$ The process of removing one $\mathrm{C}_{12}$ molecule from the system is performed in five steps shown in Figure S1. The final expression for the change of the Gibbs free energy during evaporation can be presented as:

$$
\Delta G_{\text {conf-rovib }}^{e v}=\sum_{j=2}^{95} y_{j}^{g} G_{\text {conf-rovib }}^{g, j}-\sum_{j=2}^{95} y_{j}^{l} G_{\text {conf-rovib }}^{l, j},
$$

where $y_{i}$ refers to the distribution functions in the gas and liquid phases. 


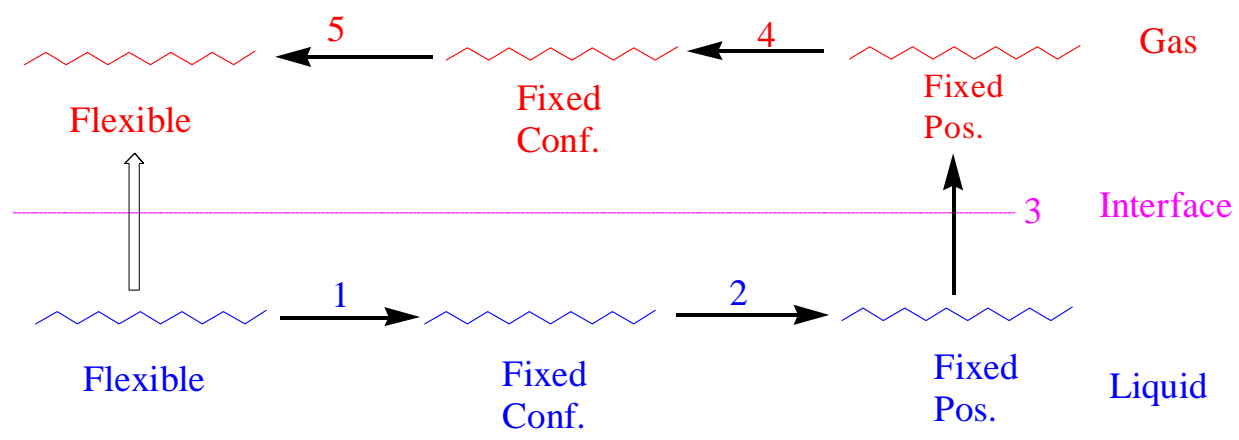

Figure S1. The five-step process of transferring a $\mathrm{C}_{12}$ molecule from the liquid to the gaseous phase (conf.=conformation, pos.=position).

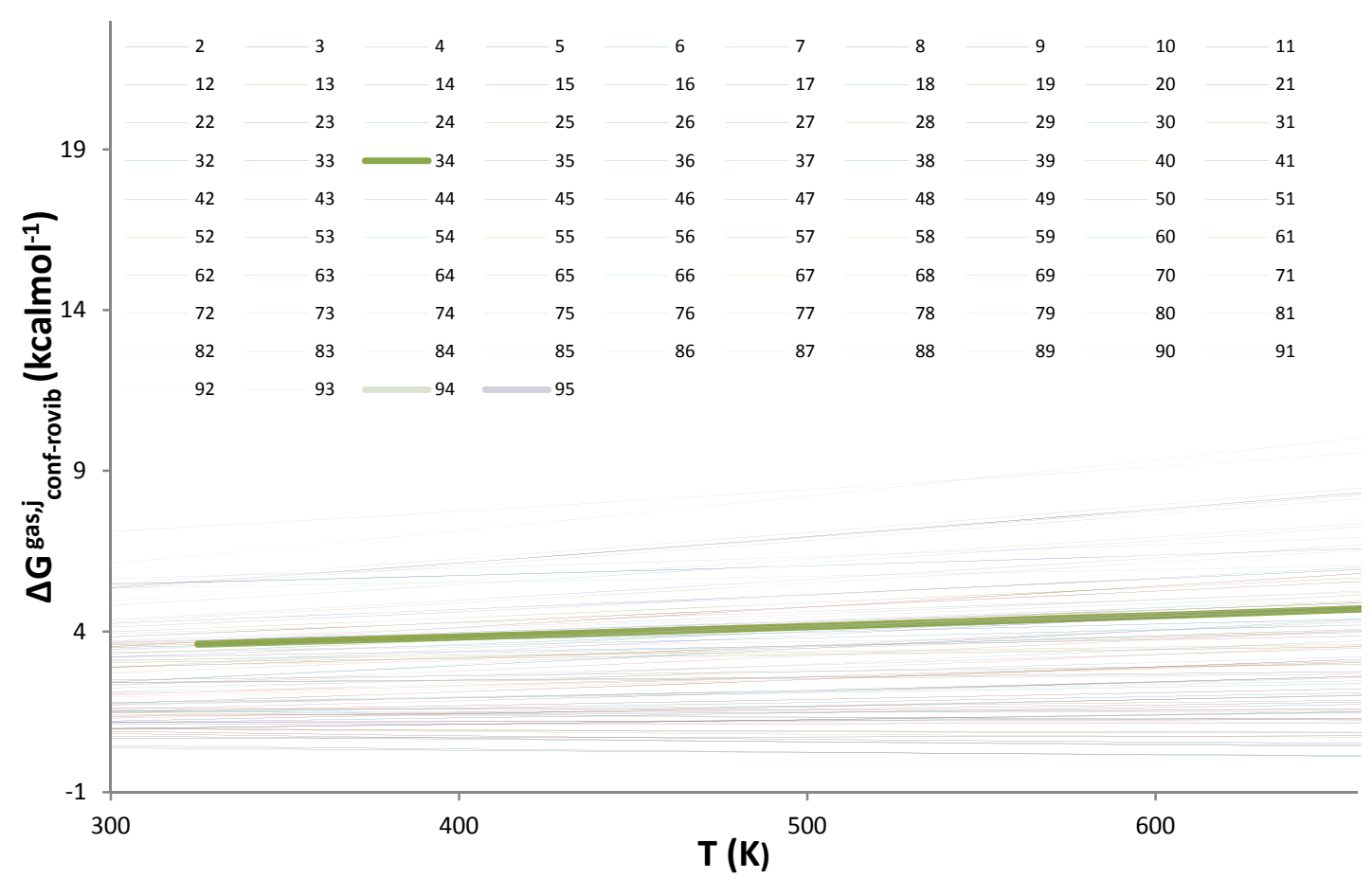

Figure S2a. Relative Gibbs free energies of $95 n$-dodecane conformers with respect to the most stable conformer (structure 1) at temperatures of 300-650 K. Optimisation and frequency calculations were performed using the $\omega \mathrm{B} 97 \mathrm{X}-\mathrm{D} / \mathrm{cc}-\mathrm{pVTZ}$ chemistry model in the gas phase. 


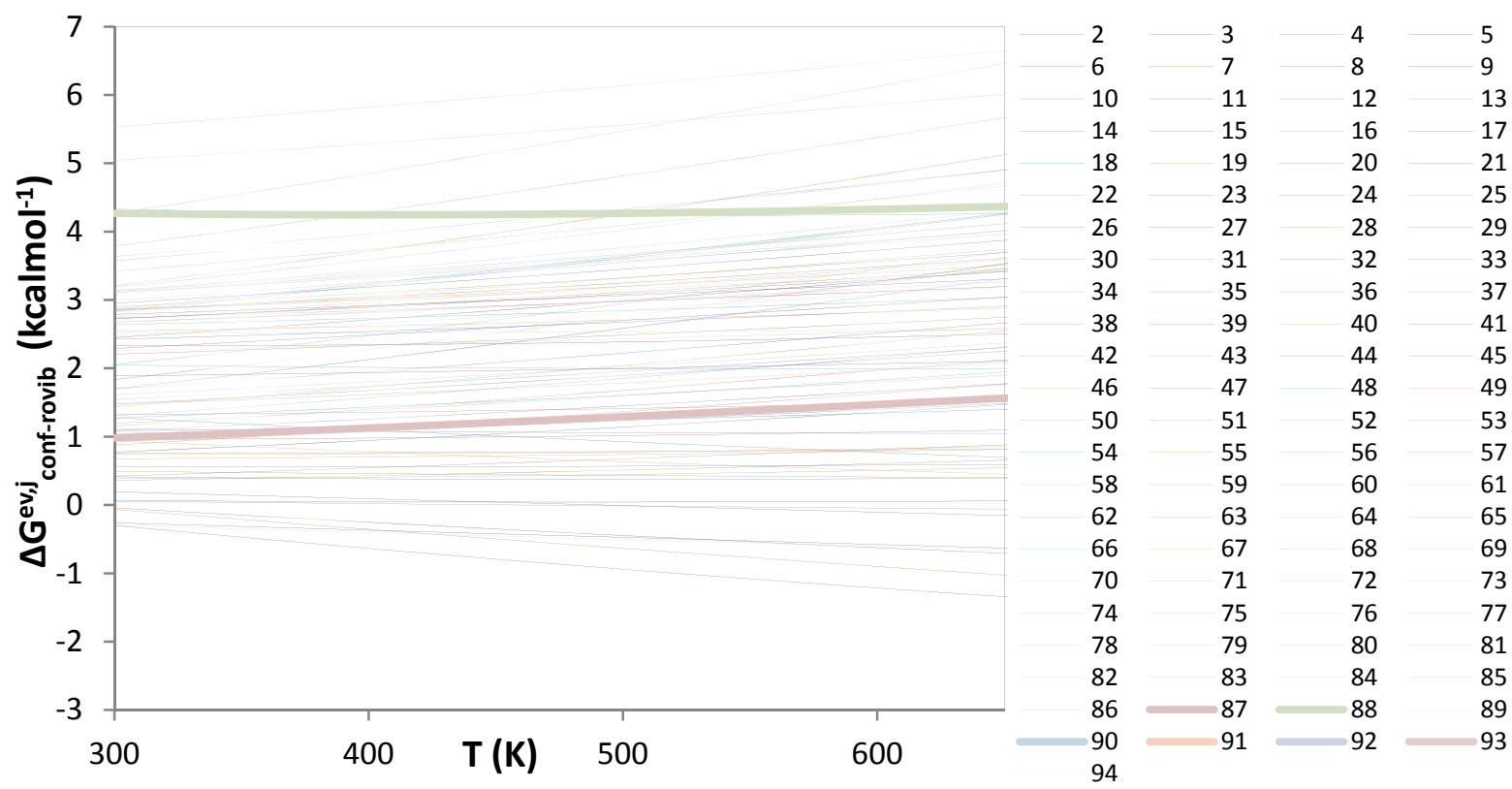

Figure S2b. The differences in the internal Gibbs free energies of $94 n$-dodecane conformers in gas and liquid phases versus temperature.

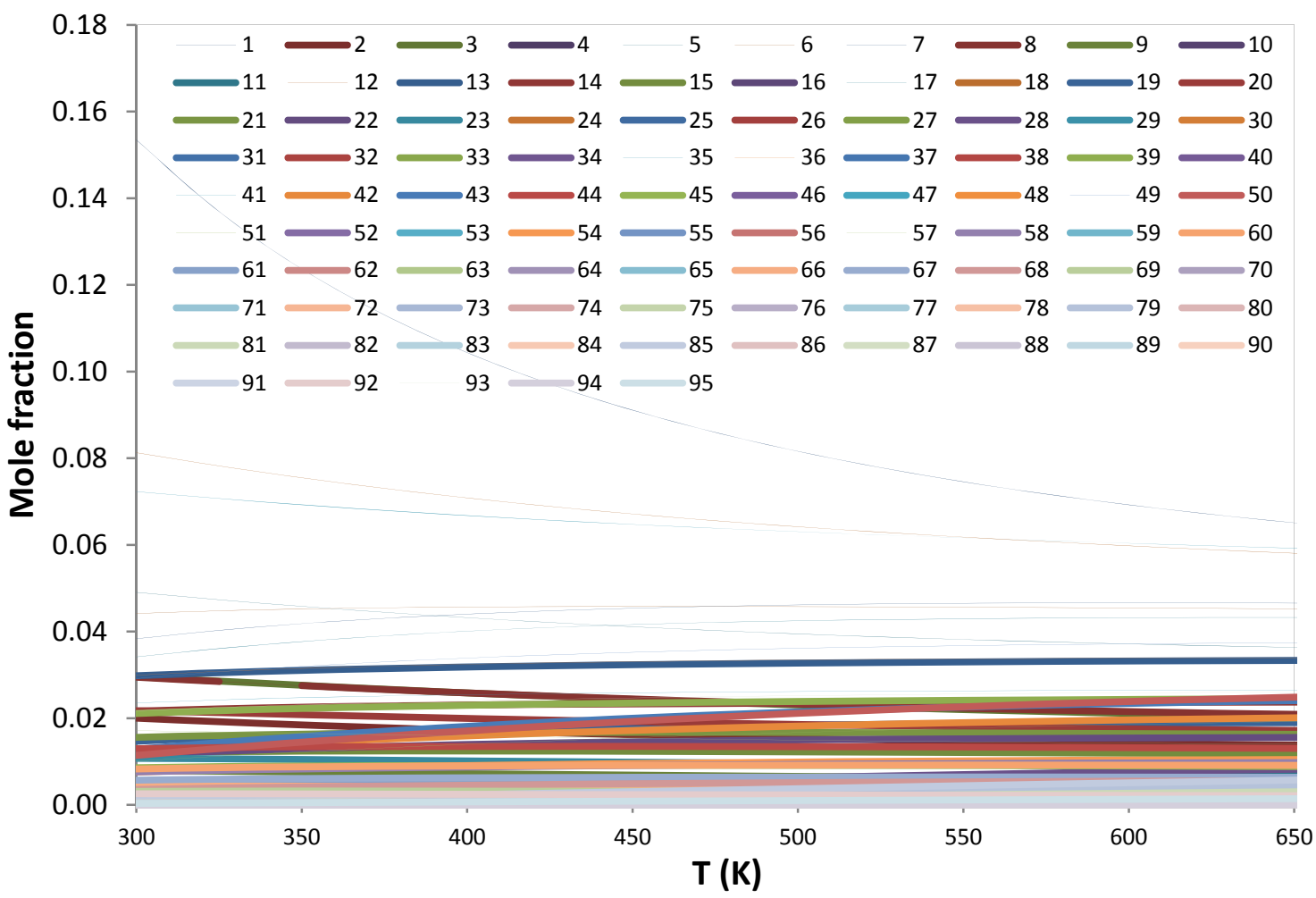

Figure S3. Mole fractions of 95 conformers of $n$-dodecane in the gas phase computed using $\omega \mathrm{B} 97 \mathrm{X}-\mathrm{D} / \mathrm{cc}-\mathrm{pVTZ}$ and MS-QH models. 


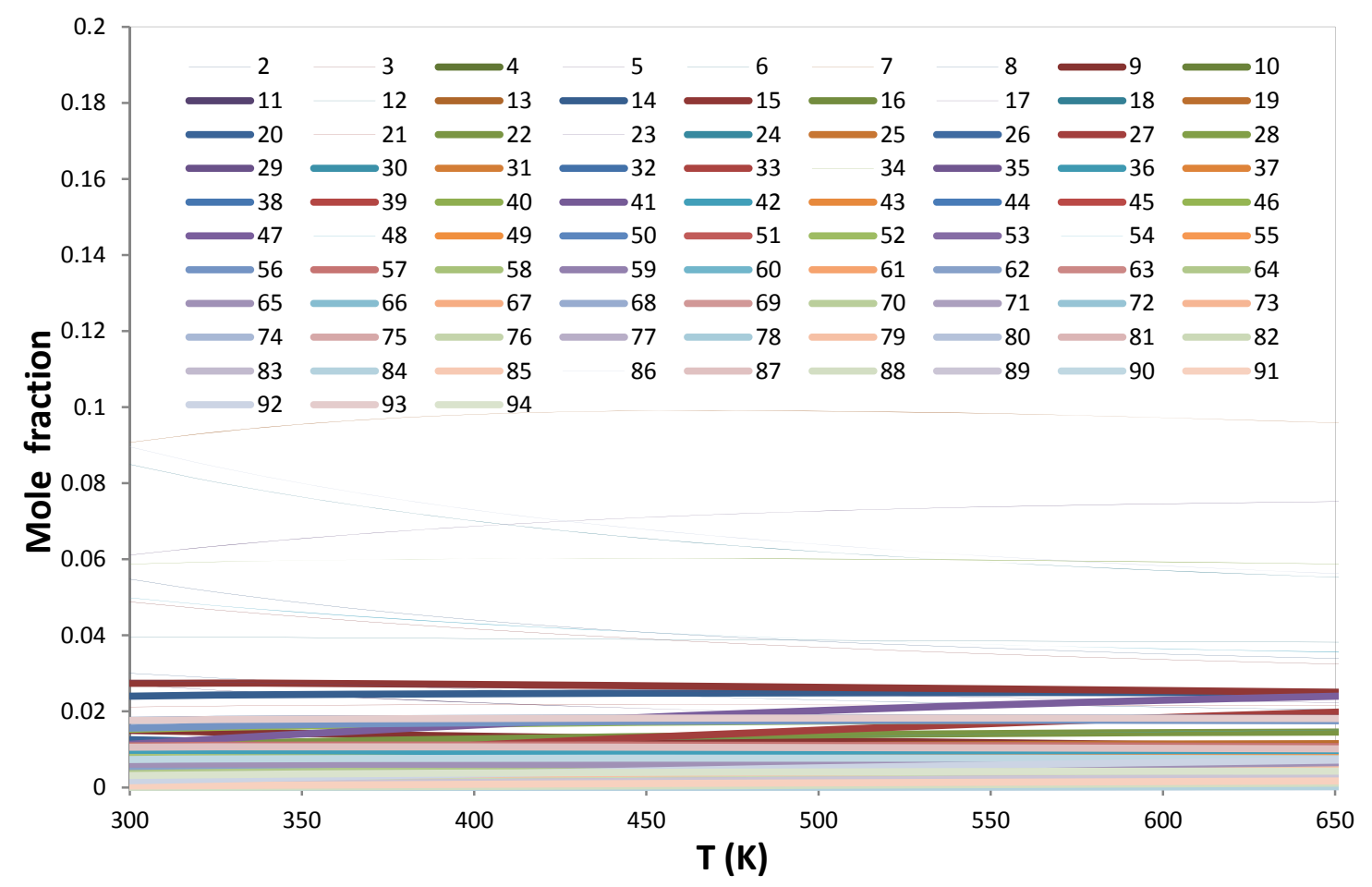

Figure S4. Mole fraction of 94 conformers of $n$-dodecane in the liquid phase computed using SMD- $\omega$ B97X-D/cc-pVTZ and MS-QH models.
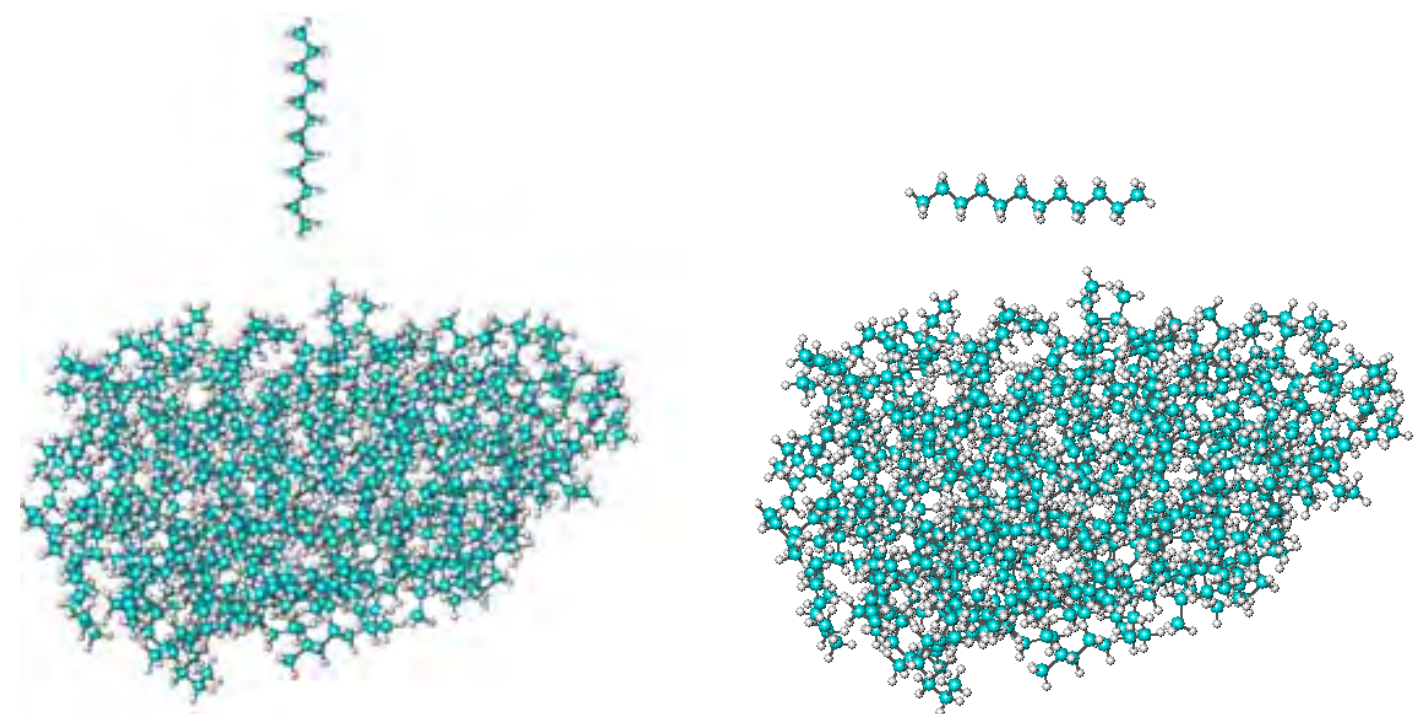

Figure S5. The initial structures of $n$-dodecane molecules attacking a nanodroplet of 70 molecules (2693 atoms). 
Zero-point energy (kcal/mol) of 95 structures in the gas phase

\begin{tabular}{|c|c|c|c|}
\hline Structure & Energy & Structure & Energy \\
\hline 1 & 226.95 & 2 & 227.64 \\
\hline 3 & 227.85 & 4 & 229.33 \\
\hline 5 & 227.63 & 6 & 228.02 \\
\hline 7 & 228.26 & 8 & 227.83 \\
\hline 9 & 228.11 & 10 & 229.28 \\
\hline 11 & 229.38 & 12 & 227.54 \\
\hline 13 & 228.09 & 14 & 228.16 \\
\hline 15 & 228.32 & 16 & 229.9 \\
\hline 17 & 228.33 & 18 & 230.13 \\
\hline 19 & 228.49 & 20 & 227.93 \\
\hline 21 & 228.33 & 22 & 228.57 \\
\hline 23 & 228.14 & 24 & 228.51 \\
\hline 25 & 229.93 & 26 & 229.46 \\
\hline 27 & 229.38 & 28 & 229.36 \\
\hline 29 & 230.76 & 30 & 229.97 \\
\hline 31 & 229.39 & 32 & 231.92 \\
\hline 33 & 229.96 & 34 & 229.93 \\
\hline 35 & 227.61 & 36 & 228.13 \\
\hline 37 & 228.63 & 38 & 230.41 \\
\hline 39 & 228.16 & 40 & 230.13 \\
\hline 41 & 228.18 & 42 & 228.68 \\
\hline 43 & 228.87 & 44 & 228.31 \\
\hline 45 & 228.43 & 46 & 229.49 \\
\hline 47 & 230.01 & 48 & 229.8 \\
\hline 49 & 228.27 & 50 & 228.94 \\
\hline 51 & 228.83 & 52 & 230.01 \\
\hline 53 & 230.13 & 54 & 228.62 \\
\hline 55 & 230.25 & 56 & 228.75 \\
\hline 57 & 227.97 & 58 & 228.54 \\
\hline 59 & 228.92 & 60 & 228.46 \\
\hline 61 & 228.37 & 62 & 229.86 \\
\hline 63 & 228.91 & 64 & 230.38 \\
\hline 65 & 228.96 & 66 & 228.32 \\
\hline 67 & 228.58 & 68 & 228.91 \\
\hline 69 & 228.48 & 70 & 228.82 \\
\hline 71 & 230.26 & 72 & 229.98 \\
\hline 73 & 230.24 & 74 & 230.09 \\
\hline 75 & 230.87 & 76 & 230.89 \\
\hline 77 & 229.97 & 78 & 230.52 \\
\hline 79 & 229.61 & 80 & 230.97 \\
\hline 81 & 230.08 & 82 & 230.69 \\
\hline
\end{tabular}




Struc
83
85
87
89
91
93
95

Energy

230.35

230.14

229.91

229.89

231

228.16

229.89
Structure

84

86

88

90

92

94

\section{Energy}

232.09

232.05

229.74

232.76

228.23

232.53

$\mathrm{ZPE}$ of the system $=\quad 226.95$ 
Optimised Cartesian coordinates of atoms (in Angstroms), electronic energies (in $\mathrm{kcal} / \mathrm{mole}$ ) with respect to structure 1 , symmetry points and moments of inertia (atomic mass times Angstroms squared) in the gas phase (6 refers to $C, 1$ refers to $H$ )

\begin{tabular}{|c|c|}
\hline & artesian coordinate of structure $\quad 1$ \\
\hline & $7.00341666-0.30791641-0.00000667$ \\
\hline & $89260510.32743515-0.00000664$ \\
\hline & $\begin{array}{lll}7.05372717 & -0.95098908 & 0.88055290\end{array}$ \\
\hline & $7.05372410-0.95098409-0.88056985$ \\
\hline & $5.722893860 .51678236-0.00000205$ \\
\hline & $5.709709041 .17362101-0.87418760$ \\
\hline & $\begin{array}{lll}5.70971396 & 1.17362001 & 0.87418424\end{array}$ \\
\hline & $4.46307194-0.340750130 .00000151$ \\
\hline & $4.47507892-0.999120420 .87470887$ \\
\hline & $4.47507661-0.99912390-0.87470419$ \\
\hline & $\begin{array}{lll}3.17555022 & 0.47332109 & 0.00000108\end{array}$ \\
\hline & $3.164104111 .13143900-0.87474606$ \\
\hline & 3.164106381 .131442740 .87474590 \\
\hline & $1.91689648-0.384930140 .00000411$ \\
\hline & $1.92828621-1.043051200 .87471750$ \\
\hline & $1.92828491-1.04305489-0.87470618$ \\
\hline & $\begin{array}{lll}0.62927634 & 0.42916554 & 0.00000328\end{array}$ \\
\hline & $0.617846341 .08728422-0.87471667$ \\
\hline & $\begin{array}{lll}0.61784631 & 1.08728688 & 0.87472165\end{array}$ \\
\hline & $\begin{array}{lll}-0.62927432 & -0.42918011 & 0.00000415\end{array}$ \\
\hline & $-0.61784432-1.087298980 .87472416$ \\
\hline & $-0.61784400-1.08730083-0.87471415$ \\
\hline & $\begin{array}{lll}-1.91689528 & 0.38491444 & 0.00000285\end{array}$ \\
\hline & $-1.928283321 .04303716-0.87470885$ \\
\hline & -1.928285121 .043037710 .87471452 \\
\hline & 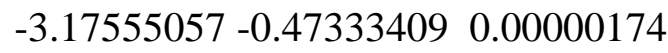 \\
\hline & $-3.16410979-1.131453990 .87474755$ \\
\hline & $-3.16410779-1.13145363-0.87474399$ \\
\hline & $\begin{array}{lll}-4.46306885 & 0.34074540 & 0.00000021\end{array}$ \\
\hline & $-4.475060790 .99912086-0.87470443$ \\
\hline & $\begin{array}{lll}-4.47506440 & 0.99911883 & 0.87470674\end{array}$ \\
\hline & $-5.72290153-0.51677029-0.00000389$ \\
\hline & $-5.70973979-1.173609840 .87418166$ \\
\hline & $-5.70973661-1.17360635-0.87419169$ \\
\hline & $\begin{array}{lll}-7.00341465 & 0.30794763-0.00000475\end{array}$ \\
\hline & $\begin{array}{ll}-7.05370966 & 0.95101730-0.88056682\end{array}$ \\
\hline & $\begin{array}{llll}-7.05371368 & 0.95101311 & 0.88056043\end{array}$ \\
\hline & $-7.88926701-0.32739845-0.00000845$ \\
\hline & energy $\quad 0.00000$ \\
\hline & Symmetry point group: $\mathrm{C} 2 \mathrm{~h}$ \\
\hline & moment of inertia $\quad 2.1947$ \\
\hline
\end{tabular}


Cartesian coordinate of structure 2

$6 \quad 6.15931299-1.64761742-0.19625843$

$17.19032805-1.562709650 .14755144$

$1 \quad 5.75710151-2.588501500 .18378565$

$1 \quad 6.17498520-1.71246825-1.28576156$

$6 \quad 5.31558662-0.467363530 .26735601$

$1 \quad 5.75494940 \quad 0.46422591-0.09994768$

$1 \quad 5.33987807-0.405449891 .35886719$

$63.86638197-0.55332747-0.19626493$

$13.42533132-1.485914830 .17042053$

$13.84050361-0.61523201-1.28908359$

$\begin{array}{lllll}6 & 3.01267483 & 0.62203736 & 0.26194797\end{array}$

$13.456093161 .55453985-0.10175728$

$13.036184380 .68127472 \quad 1.35488107$

$\begin{array}{llll}6 & 1.56495530 & 0.53708011 & -0.20519927\end{array}$

$1 \quad 1.13317189-0.402502580 .14962655$

$1 \quad 1.53936500 \quad 0.49106095-1.29907730$

$\begin{array}{lllll}6 & 0.71526260 & 1.71216291 & 0.26684228\end{array}$

$1 \quad 1.205358492 .64216755-0.03455707$

$\begin{array}{lllll}1 & 0.69192779 & 1.72167984 & 1.36143637\end{array}$

$\begin{array}{lllll}6 & -0.71526271 & 1.71216312 & -0.26684193\end{array}$

$1-0.691928001 .72168194-1.36143600$

$\begin{array}{lllll}1 & -1.20535887 & 2.64216709 & 0.03455906\end{array}$

$\begin{array}{lllll}6 & -1.56495495 & 0.53707922 & 0.20519774\end{array}$

$\begin{array}{lllll}1 & -1.53936311 & 0.49105717 & 1.29907562\end{array}$

$1-1.13317223-0.40250261-0.14963116$

$\begin{array}{lllll}6 & -3.01267517 & 0.62203793 & -0.26194712\end{array}$

$\begin{array}{llll}1 & -3.03618640 & 0.68127857 & -1.35488000\end{array}$

$\begin{array}{lllll}1 & -3.45609290 & 1.55453934 & 0.10176161\end{array}$

$\begin{array}{llll}6 & -3.86638161 & -0.55332824 & 0.19626360\end{array}$

$1-3.84050116-0.61523627 \quad 1.28908201$

$1-3.42533180-1.48591449-0.17042568$

$6-5.31558715-0.46736264-0.26735423$

$1-5.33988077-0.40544568-1.35886517$

$\begin{array}{lllll}1 & -5.75494908 & 0.46422571 & 0.09995322\end{array}$

$\begin{array}{lllll}6 & -6.15931278 & -1.64761788 & 0.19625829\end{array}$

$1-6.17498282-1.712472021 .28576124$

$1-5.75710225-2.58850084-0.18378957$

$1-7.19032847-1.56270885-0.14754924$ energy $\quad 0.434306$

Symmetry point group: C2

moment of inertia $\quad 2.2405$

Cartesian coordinate of structure 3

$6-5.05330900-2.32181978-0.05722552$

$1-5.47077352-3.022107280 .66645492$

$1-5.87952981-1.74955667-0.48302871$

$1-4.60523558-2.90367590-0.86477694$

$\begin{array}{lllll}6 & -4.02540926 & -1.39943215 & 0.58495675\end{array}$ 


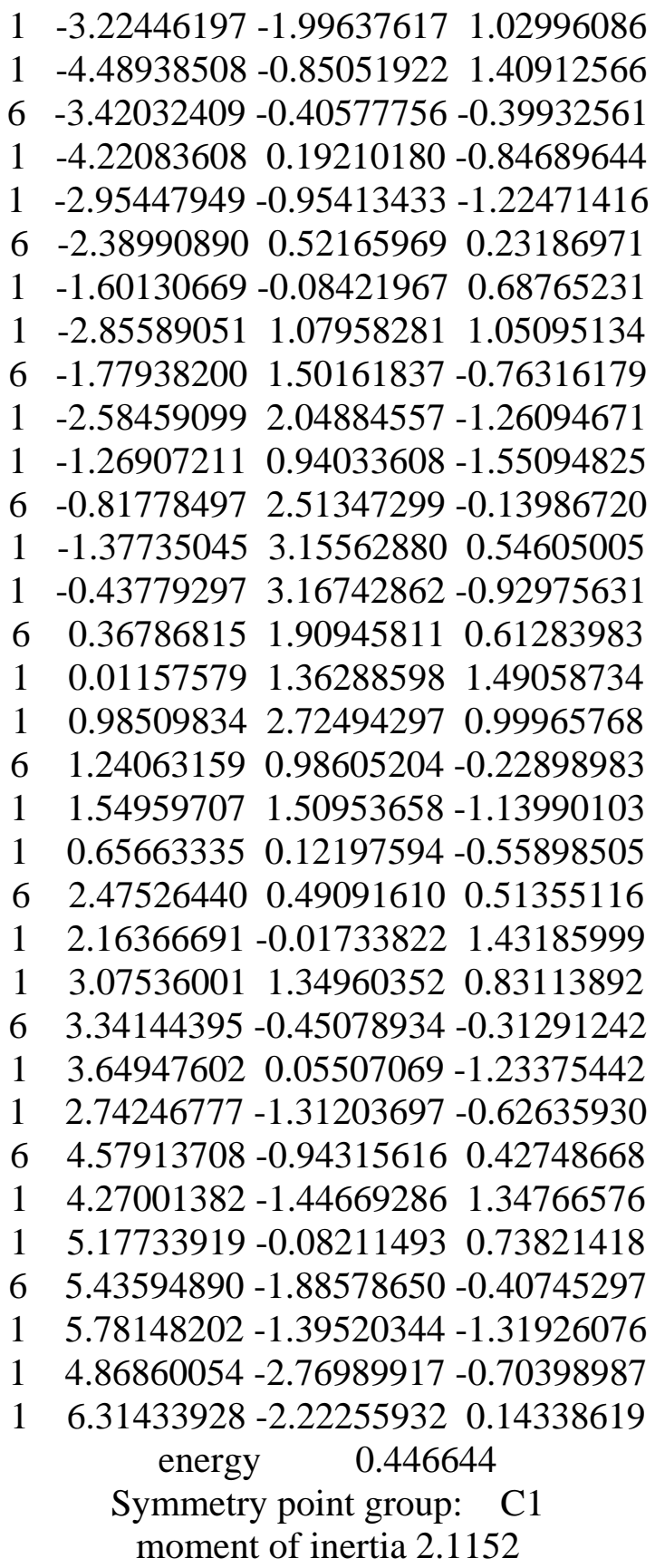

Cartesian coordinate of structure 4

$64.713707642 .52897694-0.27414076$

$\begin{array}{lllll}1 & 4.99984302 & 3.39510186 & 0.32283059\end{array}$

$1 \quad 5.62406977 \quad 1.98265466-0.52759574$

$14.279098032 .89482098-1.20621977$

$\begin{array}{lllll}6 & 3.73140417 & 1.63501602 & 0.47130560\end{array}$

$\begin{array}{llll}1 & 2.84201346 & 2.21162423 & 0.74025702\end{array}$

$\begin{array}{lllll}1 & 4.17733680 & 1.30640010 & 1.41414087\end{array}$

$6 \quad 3.309133420 .41251056-0.33467106$

$14.19814268-0.16693635-0.60476975$

$12.863314690 .74039450-1.27954383$

$\begin{array}{llll}6 & 2.32393036 & -0.48643352 & 0.40045923\end{array}$ 
$1 \quad 1.43619990 \quad 0.093264350 .66405115$

$1 \quad 2.76577609-0.808043331 .34896384$

$6 \quad 1.92602457-1.72295387-0.40132323$

$12.83112253-2.30291021-0.59816165$

$1 \quad 1.55081018-1.41421503-1.38271756$

$\begin{array}{llll}6 & 0.88324040 & -2.61731728 & 0.28337119\end{array}$

$1 \quad 0.98149526-2.517424881 .36893891$

$1 \quad 1.10054324-3.662987090 .05727989$

$6-0.56611251-2.34535975-0.11988486$

$\begin{array}{lllll}1 & -1.21206003 & -3.06417627 & 0.39266857\end{array}$

$1-0.67894335-2.54664921-1.19019573$

$\begin{array}{lllll}6 & -1.07382154 & -0.93773071 & 0.17058471\end{array}$

$1-0.49881656-0.20984388-0.40869895$

$\begin{array}{lllll}1 & -0.89938397 & -0.69711543 & 1.22441347\end{array}$

$6-2.55286252-0.76197753-0.14961648$

$1-3.13784526-1.476238250 .43857971$

$1-2.72772494-1.01861135-1.19947868$

$\begin{array}{lllll}6 & -3.06497965 & 0.64787931 & 0.11567597\end{array}$

$\begin{array}{lllll}1 & -2.48213908 & 1.36182608 & -0.47539216\end{array}$

$\begin{array}{lllll}1 & -2.88665070 & 0.90633941 & 1.16455969\end{array}$

$\begin{array}{lllll}6 & -4.54519501 & 0.82519280 & -0.20027668\end{array}$

$\begin{array}{lllll}1 & -5.12612423 & 0.11217209 & 0.39125538\end{array}$

$\begin{array}{lllll}1 & -4.72181082 & 0.56633740 & -1.24795002\end{array}$

$\begin{array}{lllll}6 & -5.04541112 & 2.23858407 & 0.06798479\end{array}$

$\begin{array}{lllll}1 & -4.49987614 & 2.96698099-0.53476893\end{array}$

$\begin{array}{lllll}1 & -4.90731333 & 2.50938098 & 1.11635194\end{array}$

$\begin{array}{lllll}1 & -6.10542651 & 2.33953137 & -0.16560886\end{array}$ energy $\quad 2.04960$

Symmetry point group: C1

moment of inertia $\quad 2.1331$

Cartesian coordinate of structure 5

$\begin{array}{llll}6 & -5.88784180 & 1.81965157 & -0.25814702\end{array}$

$\begin{array}{lllll}1 & -6.90872958 & 1.99684789 & 0.08070525\end{array}$

$\begin{array}{lllll}1 & -5.27265773 & 2.65329219 & 0.08543825\end{array}$

$1-5.890050961 .83999830-1.34951353$

$\begin{array}{lllll}6 & -5.34793326 & 0.49348768 & 0.26093750\end{array}$

$1-5.99827519-0.32164132-0.06837826$

$\begin{array}{lllll}1 & -5.38156786 & 0.48659136 & 1.35399895\end{array}$

$\begin{array}{lllll}6 & -3.92148104 & 0.21192988 & -0.19627676\end{array}$

$\begin{array}{llll}1 & -3.27877113 & 1.03635110 & 0.12423889\end{array}$

$1 \quad-3.88719196 \quad 0.20632827-1.29102641$

$\begin{array}{llll}6 & -3.37478506 & -1.11085338 & 0.32964285\end{array}$

$\begin{array}{llll}1 & -4.07180458 & -1.90960053 & 0.06097444\end{array}$

$1-3.35360982-1.081042141 .42393486$

$6-1.98542170-1.47202824-0.19063330$

$1-2.01103749-1.52032025-1.28415331$

$\begin{array}{llll}1 & -1.72996799 & -2.47868368 & 0.15187973\end{array}$

$\begin{array}{llll}6 & -0.88085192 & -0.51374924 & 0.24203752\end{array}$ 
$1-0.89326956-0.419503531 .33305562$

$1-1.078351080 .48645316-0.15260795$

$6 \quad 0.50493652-0.95720450-0.20980198$

$1 \quad 0.51244764-1.06313561-1.29941171$

$1 \quad 0.71564494-1.953068140 .19300716$

$\begin{array}{lllll}6 & 1.61297444 & 0.00025777 & 0.20984462\end{array}$

$\begin{array}{lllll}1 & 1.60375966 & 0.10826563 & 1.29919186\end{array}$

$1 \quad 1.403318700 .99539832-0.19525650$

$62.99916704-0.44369137-0.23978091$

$13.00818249-0.55192682-1.32911499$

$13.20905086-1.438730340 .16551608$

$\begin{array}{lllll}6 & 4.10697312 & 0.51407220 & 0.17958685\end{array}$

$\begin{array}{lllll}1 & 4.09845686 & 0.62267321 & 1.26901634\end{array}$

$13.897631951 .50943319-0.22556373$

$\begin{array}{lllll}6 & 5.49413711 & 0.07094697 & -0.26976783\end{array}$

$15.50121708-0.03687185-1.35795235$

$1 \quad 5.70193707-0.923157120 .13568074$

$\begin{array}{lllll}6 & 6.59335532 & 1.03588319 & 0.15503324\end{array}$

$\begin{array}{lllll}1 & 6.62603470 & 1.13693467 & 1.24139749\end{array}$

$1 \quad 6.423684682 .02977872-0.26310713$

$17.574545710 .69712012-0.17799848$ energy $\quad 0.444022$

Symmetry point group: C1 moment of inertia 2.3128

Cartesian coordinate of structure 6

$\begin{array}{llll}6 & 5.71833950 & 1.67489142 & -0.61893751\end{array}$

$1 \quad 6.796110901 .76425132-0.48165241$

$15.529765651 .50902004-1.68118966$

$15.267038582 .63169192-0.34990703$

$\begin{array}{lllll}6 & 5.13882514 & 0.54396997 & 0.22064773\end{array}$

$1 \quad 5.368910840 .71528013 \quad 1.27589754$

$15.62514109-0.39812214-0.04754042$

$\begin{array}{lllll}6 & 3.63134166 & 0.39248828 & 0.05507778\end{array}$

$1 \quad 3.40592866 \quad 0.23527017-1.00322303$

$\begin{array}{lllll}1 & 3.14065188 & 1.33086958 & 0.33510141\end{array}$

$\begin{array}{llll}6 & 3.04897340 & -0.74562140 & 0.88629391\end{array}$

$13.35204541-0.609701081 .92827392$

$13.49306888-1.691968830 .56057880$

$\begin{array}{lllll}6 & 1.52723627 & -0.85494084 & 0.82391592\end{array}$

$\begin{array}{llll}1 & 1.09102417 & 0.08317338 & 1.17793492\end{array}$

$1 \quad 1.19753776-1.628800311 .52352517$

$6 \quad 0.98352398-1.18813255-0.56332412$

$11.52886815-2.05239278-0.95405228$

$1 \quad 1.18737104-0.36198500-1.25004974$

$6-0.51246065-1.49273017-0.58880029$

$1-0.72071445-2.338429890 .07472168$

$1-0.79051678-1.81937381-1.59477091$

$\begin{array}{lll}6 & -1.39942142-0.31675518-0.19380380\end{array}$ 


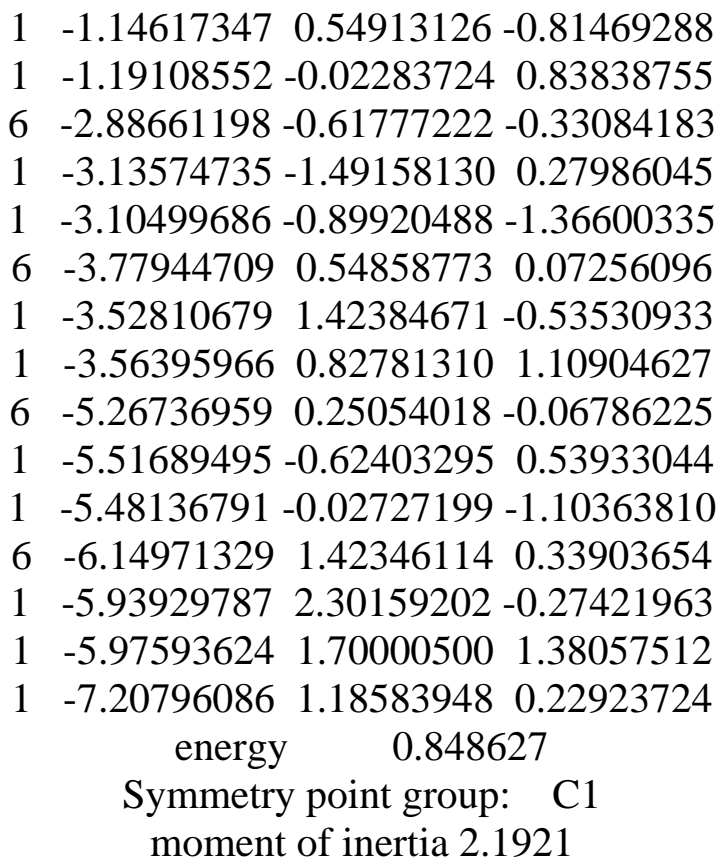

Cartesian coordinate of structure 7

$\begin{array}{llll}6 & -6.47527209 & 0.43428304 & -0.08964214\end{array}$

$\begin{array}{lllll}1 & -7.44918381 & 0.01789202 & 0.16831605\end{array}$

$\begin{array}{lllll}1 & -6.33060518 & 1.33818942 & 0.50491418\end{array}$

$1 \quad-6.506966890 .73220605-1.13928159$

$\begin{array}{lllll}6 & -5.35476657 & -0.56690107 & 0.15890003\end{array}$

$1-5.53903858-1.47526046-0.42141708$

$1-5.36094276-0.870806921 .20940344$

$6-3.97721573-0.01807378-0.19382876$

$\begin{array}{lllll}1 & -3.80478922 & 0.89644586 & 0.37992011\end{array}$

$1 \quad-3.964993630 .27667165-1.24861024$

$\begin{array}{lllll}6 & -2.85332263 & -1.01684182 & 0.06077227\end{array}$

$1-3.08784986-1.94667013-0.46498731$

$\begin{array}{llll}1 & -2.83004807 & -1.26834335 & 1.12618179\end{array}$

$6-1.46815497-0.54152397-0.37369395$

$1-1.49208913-0.28348925-1.43788735$

$1-0.77073991-1.37620953-0.27761156$

$\begin{array}{lllll}6 & -0.94038845 & 0.65298327 & 0.41644595\end{array}$

$\begin{array}{lllll}1 & -0.91626588 & 0.39487590 & 1.48062277\end{array}$

$\begin{array}{lllll}1 & -1.63791480 & 1.48760209 & 0.32053374\end{array}$

$\begin{array}{lllll}6 & 0.44461602 & 1.12850227 & -0.01817733\end{array}$

$\begin{array}{lllll}1 & 0.67910683 & 2.05832905 & 0.50754048\end{array}$

$1 \quad 0.421260831 .38001427-1.08356467$

$\begin{array}{lllll}6 & 1.56868266 & 0.12975348 & 0.23633818\end{array}$

$11.39559927-0.78451098-0.33733076$

$1 \quad 1.55611078-0.164495191 .29109518$

$6 \quad 2.945097550 .67948134-0.11694531$

$13.13154023 \quad 1.588728260 .46354656$

$1 \quad 2.95279136 \quad 0.98432947-1.16841184$

$\begin{array}{llll}6 & 4.07430634-0.31333283 & 0.12689643\end{array}$ 
$\begin{array}{cccc}1 & 3.89010344 & -1.22163224 & -0.45599822 \\ 1 & 4.06533789 & -0.62083590 & 1.17766934 \\ 6 & 5.45166430 & 0.23693560 & -0.22323935 \\ 1 & 5.63449414 & 1.14378219 & 0.35986870 \\ 1 & 5.45909061 & 0.54366835 & -1.27285514 \\ 6 & 6.57259549 & -0.76407810 & 0.02434795 \\ 1 & 6.42803150 & -1.66750355 & -0.57096917 \\ 1 & 6.60458964 & -1.06294711 & 1.07369556 \\ 1 & 7.54631961 & -0.34715443 & -0.23342683 \\ & \text { energy } & 1.02496\end{array}$

Symmetry point group: C1

moment of inertia $\quad 2.1606$

Cartesian coordinate of structure 8

$\begin{array}{llll}6 & -4.53790843 & 2.51714190 & -0.46687381\end{array}$

$\begin{array}{lllll}1 & -5.11444670 & 3.28950489 & 0.04250026\end{array}$

$\begin{array}{llll}1 & -3.63046610 & 2.98113678 & -0.85774845\end{array}$

$\begin{array}{lllll}1 & -5.12424768 & 2.17084284 & -1.31996820\end{array}$

$\begin{array}{lllll}6 & -4.20056924 & 1.36249027 & 0.46724773\end{array}$

$\begin{array}{lllll}1 & -5.12267983 & 0.93494171 & 0.87051584\end{array}$

$\begin{array}{lllll}1 & -3.63849363 & 1.73844967 & 1.32679546\end{array}$

$\begin{array}{lllll}6 & -3.39388363 & 0.26295490 & -0.21303240\end{array}$

$\begin{array}{lllll}1 & -2.48081578 & 0.70060657 & -0.62743301\end{array}$

$1-3.95846529-0.12597755-1.06718404$

$\begin{array}{lllll}6 & -3.03898928 & -0.88541548 & 0.72402572\end{array}$

$1-3.95574954-1.261808471 .18614884$

$1-2.42722592-0.501142431 .54511391$

$\begin{array}{llll}6 & -2.32284568 & -2.05322934 & 0.04538250\end{array}$

$1-3.00333838-2.51247951-0.67708336$

$1-2.11267121-2.819042510 .79724881$

6 -1.02044346 -1.69345522 -0.66973364

$1-1.23123127-1.03369337-1.51609802$

$1-0.59771991-2.60517868-1.10063880$

$\begin{array}{lllll}6 & 0.02579917 & -1.03850779 & 0.22438126\end{array}$

$1 \quad 0.20047741-1.670464141 .10153868$

$\begin{array}{lllll}1 & -0.35590625 & -0.08740408 & 0.60698147\end{array}$

$61.34772365-0.78691344-0.48965050$

$11.16734126-0.16523081-1.37262336$

$1 \quad 1.74397272-1.73637978-0.86348798$

$\begin{array}{llll}6 & 2.39392340 & -0.11677700 & 0.39175938\end{array}$

$\begin{array}{llll}1 & 2.57360284 & -0.73788247 & 1.27516800\end{array}$

$\begin{array}{lllll}1 & 1.99746939 & 0.83313468 & 0.76455713\end{array}$

$\begin{array}{llll}6 & 3.71529264 & 0.13580125 & -0.32277401\end{array}$

$13.536027040 .75761460-1.20592186$

$14.11204181-0.81371081-0.69653466$

$\begin{array}{llll}6 & 4.76305228 & 0.80540393 & 0.55829505\end{array}$

$\begin{array}{lllll}1 & 4.94173917 & 0.18280263 & 1.43935501\end{array}$

$14.36539715 \quad 1.753315910 .93133806$

$6 \quad 6.079139751 .05374161-0.16702545$ 
$1 \quad 5.931207651 .69965762-1.03442046$

$1 \quad 6.511120980 .11702472-0.52398065$

$1 \quad 6.81131305 \quad 1.53194846 \quad 0.48385035$ energy $\quad 0.460547$

Symmetry point group: C1 moment of inertia 2.2073

Cartesian coordinate of structure 9

$65.06700809-1.83189495-0.60211598$

$15.47043675-2.82351811-0.39636942$

$14.71240344-1.82333682-1.63442965$

$15.88782852-1.11598289-0.53002074$

$6 \quad 3.94457675-1.472698650 .36261942$

$14.31487668-1.519224261 .39047059$

$1 \quad 3.14800955-2.218810690 .29233630$

$6 \quad 3.35680403-0.090705480 .10371095$

$12.99866900-0.04723800-0.92897067$

$\begin{array}{lllll}1 & 4.14933960 & 0.66114021 & 0.18195981\end{array}$

$\begin{array}{lllll}6 & 2.22613762 & 0.26729614 & 1.06107990\end{array}$

$\begin{array}{lllll}1 & 2.58452475 & 0.15824954 & 2.08834889\end{array}$

$1 \quad 1.41803692-0.461910090 .94823854$

$\begin{array}{lllll}6 & 1.67624326 & 1.68194699 & 0.88485798\end{array}$

$\begin{array}{llll}1 & 2.47081080 & 2.40057104 & 1.10381785\end{array}$

$1 \quad 0.90146703 \quad 1.853426361 .63567163$

$6 \quad 1.11473837 \quad 1.99201035-0.50531164$

$1 \quad 1.931752332 .01387469-1.23018863$

$1 \quad 0.698778233 .00319003-0.49122540$

$\begin{array}{llll}6 & 0.04299033 & 1.02290459-1.00212634\end{array}$

$1 \quad 0.473036350 .02400321-1.12341788$

$\begin{array}{llll}1 & -0.27261369 & 1.33461840-2.00151425\end{array}$

$\begin{array}{llll}6 & -1.18461142 & 0.93053073 & -0.10336256\end{array}$

$\begin{array}{lllll}1 & -1.59403808 & 1.93394783 & 0.05377815\end{array}$

$\begin{array}{lllll}1 & -0.89493289 & 0.56284492 & 0.88515396\end{array}$

$\begin{array}{llll}6 & -2.27065790 & 0.02348655 & -0.66762855\end{array}$

$1-1.85518330-0.97602879-0.83228362$

$\begin{array}{lllll}1 & -2.57447249 & 0.39147820 & -1.65284539\end{array}$

$\begin{array}{lllll}6 & -3.49661163 & -0.08342879 & 0.22998956\end{array}$

$\begin{array}{lllll}1 & -3.91236927 & 0.91582050 & 0.39539082\end{array}$

$\begin{array}{lllll}1 & -3.19275390 & -0.45186869 & 1.21520971\end{array}$

$6-4.58290844-0.99229636-0.33247331$

$1-4.16635220-1.99003966-0.49621979$

$1-4.88513822-0.62363339-1.31662678$

$\begin{array}{lllll}6 & -5.80364459 & -1.09067733 & 0.57303374\end{array}$

$\begin{array}{lllll}1 & -6.25619150 & -0.10930128 & 0.72650409\end{array}$

$1-5.53156163-1.484776141 .55391634$

$1-6.56474959-1.746338980 .14967653$

energy $\quad 0.498402$

Symmetry point group: C1

moment of inertia $\quad 2.1060$ 
Cartesian coordinate of structure $\quad 10$

$\begin{array}{llll}6 & -2.47537337 & 3.28729889 & -0.58315434\end{array}$

$\begin{array}{llll}1 & -2.28325599 & 4.26592709-0.14295354\end{array}$

$\begin{array}{llll}1 & -1.74950597 & 3.13283119 & -1.38362557\end{array}$

$1-3.467078443 .31474769-1.03831068$

$\begin{array}{lllll}6 & -2.38300663 & 2.17781704 & 0.45590324\end{array}$

$\begin{array}{lllll}1 & -3.09466451 & 2.36916626 & 1.26384567\end{array}$

$\begin{array}{lllll}1 & -1.39050546 & 2.18937135 & 0.91617666\end{array}$

$\begin{array}{lllll}6 & -2.64177654 & 0.79379162 & -0.12640968\end{array}$

$\begin{array}{llll}1 & -1.92355677 & 0.61163292 & -0.93032316\end{array}$

$\begin{array}{lllll}1 & -3.63128923 & 0.77324821 & -0.59467082\end{array}$

$\begin{array}{llll}6 & -2.55592763 & -0.31617433 & 0.91421872\end{array}$

$1-3.31280493-0.135422491 .68254296$

$\begin{array}{lllll}1 & -1.59325183 & -0.25864170 & 1.43036493\end{array}$

$\begin{array}{llll}6 & -2.75753027 & -1.72340382 & 0.35559674\end{array}$

$1-3.74994549-1.78123459-0.10113261$

$\begin{array}{lllll}1 & -2.76587820 & -2.42981837 & 1.19044492\end{array}$

$6-1.72388041-2.20453150-0.66689850$

$1-1.77593724-1.59229451-1.57137097$

$1-2.01895223-3.20961405-0.97475527$

$6-0.27474395-2.24292714-0.16433609$

$1 \quad 0.24937233-3.07223342-0.64642269$

$\begin{array}{llll}1 & -0.26803261-2.46841537 & 0.90730385\end{array}$

$6 \quad 0.53212602-0.97197871-0.41862759$

$1 \quad 0.04945691-0.112994430 .05223646$

$1 \quad 0.53614073-0.76361291-1.49343472$

$6 \quad 1.96782496-1.06525746 \quad 0.08097802$

$12.46421010-1.91640809-0.39619971$

$1 \quad 1.96285168-1.279860931 .15455171$

$\begin{array}{lllll}6 & 2.77714834 & 0.19962001 & -0.17479189\end{array}$

$\begin{array}{lllll}1 & 2.27932882 & 1.04986681 & 0.30292714\end{array}$

$1 \quad 2.78169767 \quad 0.41565510-1.24811490$

$\begin{array}{lllll}6 & 4.21381042 & 0.11328209 & 0.32601398\end{array}$

$14.71005073-0.73559550-0.15245815$

$14.20754334-0.102771021 .39796784$

$\begin{array}{lllll}6 & 5.01149257 & 1.38439996 & 0.06576531\end{array}$

14.551134622 .242255590 .55934279

$1 \quad 5.05758147 \quad 1.60479839-1.00230214$

$\begin{array}{lllll}1 & 6.03430956 & 1.29779689 & 0.43282255\end{array}$

energy $\quad 1.72379$

Symmetry point group: C1 moment of inertia 2.1559

Cartesian coordinate of structure 11

$\begin{array}{llll}6 & 3.84112075 & 2.88088146 & 0.01257595\end{array}$

$14.660129463 .55491234-0.23900497$

$12.979915463 .15973490-0.59740905$ 
13.574866553 .054201261 .05687806

$\begin{array}{lllll}6 & 4.22340287 & 1.42467567 & -0.21761097\end{array}$

$\begin{array}{lllll}1 & 5.10670962 & 1.17829871 & 0.37803635\end{array}$

$14.51183708 \quad 1.28177894-1.26279722$

$\begin{array}{lllll}6 & 3.10018177 & 0.45400211 & 0.12747838\end{array}$

$1 \quad 2.21626594 \quad 0.71529026-0.46134907$

$\begin{array}{lllll}1 & 2.81806025 & 0.58497626 & 1.17745677\end{array}$

$63.47515029-1.00237291-0.12075958$

$14.41347573-1.21640007 \quad 0.39930051$

$13.68309440-1.13591556-1.18741484$

$\begin{array}{llll}6 & 2.43190868 & -2.03300942 & 0.31147077\end{array}$

$1 \quad 2.29163024-1.972948601 .39535841$

$1 \quad 2.84438029-3.024844840 .11677536$

$6 \quad 1.06794608-1.90638685-0.38096279$

$10.64894944-2.90221098-0.54557215$

$1 \quad 1.20565967-1.47275975-1.37709003$

$\begin{array}{lllll}6 & 0.03916331 & -1.08451469 & 0.39122885\end{array}$

$1 \quad 0.44252274-0.095543840 .62090122$

$1-0.14460274-1.566153251 .35690122$

$6-1.28077491-0.92687815-0.35196995$

$1-1.68577729-1.91677435-0.58603853$

$1-1.09643471-0.43972874-1.31499291$

$\begin{array}{lllll}6 & -2.31990968 & -0.12791958 & 0.42434444\end{array}$

$\begin{array}{lllll}1 & -1.91400876 & 0.86173777 & 0.65732171\end{array}$

$1-2.50409176-0.614449201 .38755296$

$\begin{array}{lllll}6 & -3.63996009 & 0.03204640 & -0.31874615\end{array}$

$1-4.04700858-0.95750772-0.55070511$

$1 \quad-3.456298910 .51746899-1.28278563$

$\begin{array}{lllll}6 & -4.67907311 & 0.83309313 & 0.45654887\end{array}$

$\begin{array}{lllll}1 & -4.27165305 & 1.82158136 & 0.68615504\end{array}$

$\begin{array}{lllll}1 & -4.86066220 & 0.34785082 & 1.41956291\end{array}$

$\begin{array}{llll}6 & -5.99506256 & 0.98496946 & -0.29507212\end{array}$

$\begin{array}{lllll}1 & -6.43748557 & 0.01025711 & -0.50876534\end{array}$

$1-5.843712721 .49400913-1.24871901$

$\begin{array}{lllll}1 & -6.72032089 & 1.56161929 & 0.27928910\end{array}$ energy $\quad 2.06355$

Symmetry point group: C1 moment of inertia 2.0883

Cartesian coordinate of structure 12

$\begin{array}{llll}6 & 6.43567255 & 1.13747772 & 0.04393427\end{array}$

$\begin{array}{lllll}1 & 6.72578776 & 2.03784589 & 0.58576977\end{array}$

$\begin{array}{lllll}1 & 7.16923981 & 0.36097912 & 0.26854827\end{array}$

$1 \quad 6.502755861 .35457944-1.02376041$

$\begin{array}{lllll}6 & 5.03019914 & 0.68616388 & 0.41993242\end{array}$

$\begin{array}{lllll}1 & 4.32664311 & 1.49691379 & 0.21914239\end{array}$

$\begin{array}{lllll}1 & 4.98039361 & 0.49678618 & 1.49623304\end{array}$

$64.59852144-0.57200031-0.32715052$

$15.35167845-1.34872805-0.16689456$ 
$14.59741586-0.36750573-1.40293445$

$6 \quad 3.23213549-1.115278610 .08391806$

$13.23765670-1.330782991 .15739983$

$13.06698153-2.07323448-0.41704371$

$62.06264812-0.18982112-0.23479413$

$12.089828690 .07099755-1.29806776$

$\begin{array}{lllll}1 & 2.16989480 & 0.75044297 & 0.31252647\end{array}$

$\begin{array}{lllll}6 & 0.70839035 & -0.80276666 & 0.09920203\end{array}$

$1 \quad 0.68637355-1.072926581 .15988907$

$10.58846012-1.73940766-0.45462396$

$\begin{array}{llll}6 & -0.46509963 & 0.11820117 & -0.20953457\end{array}$

$\begin{array}{llll}1 & -0.44216407 & 0.38951203 & -1.26986780\end{array}$

$\begin{array}{lllll}1 & -0.34578631 & 1.05419534 & 0.34550139\end{array}$

$\begin{array}{lllll}6 & -1.81942858 & -0.49460356 & 0.12355779\end{array}$

$1-1.84252975-0.765161391 .18405610$

$1-1.93864140-1.43098797-0.43081870$

$\begin{array}{lllll}6 & -2.99290934 & 0.42613252 & -0.18626609\end{array}$

$1-2.970159820 .69591016-1.24700045$

$\begin{array}{lllll}1 & -2.87316882 & 1.36291334 & 0.36740127\end{array}$

$\begin{array}{llll}6 & -4.34718901 & -0.18591738 & 0.14797730\end{array}$

$\begin{array}{lllll}1 & -4.37079154 & -0.45515129 & 1.20895646\end{array}$

$1-4.46729423-1.12323040-0.40494969$

$\begin{array}{lllll}6 & -5.52180339 & 0.73404781 & -0.16259905\end{array}$

$1-5.497410761 .00145714-1.22265996$

$\begin{array}{lllll}1 & -5.40035978 & 1.66987959 & 0.38993388\end{array}$

$\begin{array}{lllll}6 & -6.87014823 & 0.11202640 & 0.17634303\end{array}$

$1-6.93011696-0.13596181 \quad 1.23765085$

$1-7.02755995-0.80986724-0.38650490$

$1-7.693060050 .78856185-0.05500565$ energy $\quad 0.474511$

Symmetry point group: C1 moment of inertia $\quad 2.2274$

Cartesian coordinate of structure 13

$\begin{array}{lllll}6 & -5.35368197 & 2.11012442 & -0.36303453\end{array}$

$1-5.580355722 .60285593-1.30869304$

$\begin{array}{lllll}1 & -6.29459516 & 1.76064107 & 0.06596252\end{array}$

$\begin{array}{lllll}1 & -4.94211979 & 2.86098835 & 0.31404724\end{array}$

$\begin{array}{llll}6 & -4.37805394 & 0.95577599 & -0.55274420\end{array}$

$1-3.464510341 .33014013-1.01961766$

$\begin{array}{lllll}1 & -4.80175521 & 0.22682434 & -1.24981580\end{array}$

$\begin{array}{lllll}6 & -4.03894820 & 0.25272185 & 0.75799992\end{array}$

$\begin{array}{lllll}1 & -4.97102450 & -0.04952772 & 1.24395011\end{array}$

$\begin{array}{lllll}1 & -3.56163365 & 0.96858374 & 1.43538654\end{array}$

$\begin{array}{lllll}6 & -3.14072047 & -0.97218213 & 0.60494897\end{array}$

$1-3.62952957-1.69873953-0.05232122$

$\begin{array}{llll}1 & -3.04146674 & -1.46174227 & 1.57786869\end{array}$

$\begin{array}{lllll}6 & -1.74606202 & -0.66896663 & 0.06696664\end{array}$

$\begin{array}{lllll}1 & -1.29328460 & 0.11355777 & 0.68241106\end{array}$ 
$1-1.81555977-0.26148001-0.94564869$

$\begin{array}{lllll}6 & -0.84537041 & -1.89953774 & 0.04107456\end{array}$

$1-1.35913011-2.69775009-0.50206957$

$1-0.70891471-2.266693531 .06350249$

$6 \quad 0.52181014-1.66814282-0.59781779$

$1 \quad 0.38481282-1.32575001-1.62873938$

$11.04866836-2.62425356-0.66284781$

$\begin{array}{llll}6 & 1.40530733 & -0.67261018 & 0.14637009\end{array}$

$1 \quad 1.49345240-0.982041151 .19318537$

$\begin{array}{lllll}1 & 0.92993900 & 0.31179271 & 0.15856921\end{array}$

$62.79704806-0.54064580-0.45933635$

$12.70678856-0.24450276-1.50944591$

$13.28523623-1.52050278-0.46166397$

$\begin{array}{lllll}6 & 3.68228561 & 0.46094666 & 0.27103601\end{array}$

$\begin{array}{llll}1 & 3.77076612 & 0.16693614 & 1.32197355\end{array}$

$\begin{array}{lllll}1 & 3.19560485 & 1.44169798 & 0.27094785\end{array}$

$\begin{array}{lllll}6 & 5.07550207 & 0.59217876 & -0.33244934\end{array}$

$14.985513010 .88537778-1.38209206$

$15.56083041-0.38775336-0.33097271$

$\begin{array}{lllll}6 & 5.95088431 & 1.59681862 & 0.40527042\end{array}$

$\begin{array}{llll}1 & 6.08028663 & 1.30891232 & 1.45017815\end{array}$

$\begin{array}{lllll}1 & 5.50109304 & 2.59128297 & 0.39109297\end{array}$

$1 \quad 6.940885501 .67225966-0.04485444$ energy $\quad 0.878084$

Symmetry point group: C1 moment of inertia $\quad 2.3239$

Cartesian coordinate of structure 14

$64.982740392 .41932411-0.20114774$

$\begin{array}{lllll}1 & 4.76158294 & 3.42678536 & 0.15174658\end{array}$

$\begin{array}{lllll}1 & 5.97226772 & 2.14494357 & 0.16907173\end{array}$

$15.036564402 .45197154-1.29094158$

$\begin{array}{lllll}6 & 3.93017806 & 1.42021374 & 0.26177797\end{array}$

$1 \quad 2.94852017 \quad 1.74344141-0.09108201$

$\begin{array}{llll}1 & 3.87820557 & 1.42057564 & 1.35449565\end{array}$

$\begin{array}{lllll}6 & 4.21470077 & 0.00170499 & -0.22246709\end{array}$

$15.22875786-0.273574550 .08111290$

$14.21176000-0.01072489-1.31745005$

$\begin{array}{lllll}6 & 3.23993140 & -1.05280099 & 0.29617881\end{array}$

$1 \quad 3.25095389-1.046412701 .39108084$

$13.59543397-2.04212239-0.00514283$

$61.80488955-0.87932325-0.19017083$

$1 \quad 1.79721155-0.84363788-1.28512136$

$\begin{array}{lllll}1 & 1.41662755 & 0.08237332 & 0.15063702\end{array}$

$\begin{array}{llll}6 & 0.88275747 & -1.99846015 & 0.28209349\end{array}$

$10.85473434-2.002183091 .37667245$

$11.31519443-2.95834496-0.01386656$

$6-0.54311045-1.91174166-0.25706219$

$\begin{array}{lllll}1 & -1.09034422 & -2.80924816 & 0.04454852\end{array}$ 
$1-0.51637252-1.92549322-1.35154039$

$\begin{array}{lllll}6 & -1.32140800 & -0.68580819 & 0.20886488\end{array}$

$\begin{array}{lllll}1 & -0.83300021 & 0.22484020 & -0.14794216\end{array}$

$\begin{array}{lllll}1 & -1.29577617 & -0.63752274 & 1.30263165\end{array}$

$6-2.77040534-0.68466592-0.26198027$

$1-3.27067754-1.586814180 .10447610$

$1-2.79471215-0.74725075-1.35471498$

$\begin{array}{lllll}6 & -3.55211365 & 0.54257131 & 0.18869077\end{array}$

$\begin{array}{lllll}1 & -3.05422239 & 1.44486757 & -0.18101554\end{array}$

$\begin{array}{lllll}1 & -3.52507197 & 0.60789538 & 1.28127979\end{array}$

$\begin{array}{lllll}6 & -5.00274234 & 0.54280877 & -0.27840374\end{array}$

$\begin{array}{lllll}1 & -5.49910354 & -0.35822542 & 0.09249263\end{array}$

$\begin{array}{llll}1 & -5.02815568 & 0.47668975 & -1.36964483\end{array}$

$\begin{array}{lllll}6 & -5.77388960 & 1.77486845 & 0.17676429\end{array}$

$1-5.314349452 .68734397-0.20753473$

$\begin{array}{lllll}1 & -5.78770641 & 1.84668945 & 1.26585610\end{array}$

$1-6.807491651 .75099049-0.16893509$ energy $\quad 0.889346$

Symmetry point group: C1 moment of inertia 2.1973

Cartesian coordinate of structure 15

$64.269011652 .67980443-0.19833667$

$13.955984403 .56762413-0.74806066$

14.531357452 .989030950 .81510200

$15.175718532 .29742842-0.67081586$

$6 \quad 3.176564141 .61849786-0.17974565$

$1 \quad 2.90888591 \quad 1.35986857-1.20640519$

$\begin{array}{lllll}1 & 2.27196644 & 2.02981018 & 0.27818651\end{array}$

$\begin{array}{lllll}6 & 3.59088425 & 0.36200556 & 0.58018802\end{array}$

13.922816930 .652892941 .58091379

$\begin{array}{lllll}1 & 4.46141018 & -0.08448012 & 0.08837458\end{array}$

$\begin{array}{llll}6 & 2.48840072-0.68614285 & 0.70857909\end{array}$

$1 \quad 1.62851416-0.231989671 .21011952$

$1 \quad 2.83349611-1.490163841 .36528234$

$6 \quad 2.04826835-1.29357191-0.62007787$

$12.93258550-1.65511631-1.15278519$

$1 \quad 1.61146738-0.51966490-1.25487728$

$61.05842458-2.44948715-0.47468447$

$1 \quad 1.55145972-3.274697240 .04683461$

$1 \quad 0.80714362-2.82305010-1.47130375$

$\begin{array}{lll}6 & -0.23543248-2.10640655 & 0.26405266\end{array}$

$\begin{array}{llll}1 & -0.01441931-1.86501751 & 1.30755083\end{array}$

$\begin{array}{lllll}1 & -0.86694478 & -2.99859665 & 0.29153148\end{array}$

$6-1.02616507-0.96016457-0.35579470$

$1-1.20053170-1.17121330-1.41615112$

$1-0.43476054-0.04041658-0.32294155$

$\begin{array}{llll}6 & -2.36005326 & -0.71213136 & 0.33689023\end{array}$

$\begin{array}{llll}1 & -2.18290043 & -0.51536175 & 1.39925572\end{array}$ 
$1-2.96548126-1.623156580 .29434498$

$\begin{array}{lllll}6 & -3.14791547 & 0.44472211 & -0.26399375\end{array}$

$1-3.323582560 .24965910-1.32692588$

$1-2.543064761 .35628056-0.21944339$

$\begin{array}{lllll}6 & -4.48268356 & 0.69373392 & 0.42795664\end{array}$

$\begin{array}{lllll}1 & -4.30551896 & 0.88842754 & 1.48934257\end{array}$

$\begin{array}{lllll}1 & -5.08578145 & -0.21731447 & 0.38249655\end{array}$

$\begin{array}{lllll}6 & -5.26122033 & 1.85256116 & -0.18097166\end{array}$

$1-5.476864931 .66722233-1.23482941$

$\begin{array}{lllll}1 & -4.69076231 & 2.78117947-0.12007055\end{array}$

$\begin{array}{lllll}1 & -6.21069439 & 2.01029080 & 0.33090321\end{array}$

energy $\quad 0.778245$

Symmetry point group: C1

moment of inertia 2.0683

Cartesian coordinate of structure $\quad 16$

$\begin{array}{rrrrr}6 & -5.17183542 & -1.97592314 & -0.42132653 \\ 1 & -5.60852858 & -2.20651868 & -1.39320454 \\ 1 & -4.92357325 & -2.92036914 & 0.06642413 \\ 1 & -5.93903270 & -1.48607255 & 0.18119122 \\ 6 & -3.93961373 & -1.09081104 & -0.55713855 \\ 1 & -4.21237490 & -0.17316603 & -1.08253253 \\ 1 & -3.19410978 & -1.59144217 & -1.18207112 \\ 6 & -3.31064882 & -0.74936375 & 0.79022723 \\ 1 & -3.11217343 & -1.68032219 & 1.32890482 \\ 1 & -4.03681106 & -0.19647450 & 1.39531734 \\ 6 & -2.01541470 & 0.05404809 & 0.69692121 \\ 1 & -1.29078833 & -0.51365299 & 0.10593392 \\ 1 & -1.58524302 & 0.15256594 & 1.69768609 \\ 6 & -2.19150550 & 1.44605314 & 0.09679608 \\ 1 & -3.01393861 & 1.94789559 & 0.61590038 \\ 1 & -2.50060131 & 1.35723331 & -0.94825739 \\ 6 & -0.95376970 & 2.34141183 & 0.15961292 \\ 1 & -0.68092064 & 2.51055170 & 1.20612452 \\ 1 & -1.23026564 & 3.31868740 & -0.24079700 \\ 6 & 0.26908813 & 1.81136480 & -0.60199183 \\ 1 & 0.81208795 & 2.64925558 & -1.04640517 \\ 1 & -0.06705312 & 1.19489519 & -1.44239046 \\ 6 & 1.25238925 & 1.01281755 & 0.24972300 \\ 1 & 0.73630620 & 0.19062696 & 0.75063920 \\ 1 & 1.63487517 & 1.65839877 & 1.04671405 \\ 6 & 2.42159853 & 0.45578071 & -0.55175964 \\ 1 & 2.93782100 & 1.27739823 & -1.05894234 \\ 1 & 2.03781319 & -0.19547212 & -1.34381082 \\ 6 & 3.42122457 & -0.32017323 & 0.29618400 \\ 1 & 2.90442111 & -1.14065824 & 0.80457626 \\ 1 & 3.80725657 & 0.33085578 & 1.08727379 \\ 6 & 4.58890004 & -0.88265351 & -0.50544538\end{array}$ 
$15.10400186-0.06215960-1.01267186$

$14.20175126-1.53288601-1.29485514$

$6 \quad 5.58150711-1.656289640 .35247656$

$15.09623813-2.500117830 .84638751$

$1 \quad 6.00520656-1.018696101 .13057145$

$16.40611674-2.04792716-0.24338067$ energy $\quad 2.54807$

Symmetry point group: C1

moment of inertia 2.1028

Cartesian coordinate of structure 17

$6-6.56387010-0.40724300 \quad 0.09004063$

$1-7.339274200 .32354590-0.14043960$

$1-6.79253843-1.32212917-0.45969701$

$1-6.62917569-0.638194371 .15493415$

$\begin{array}{lllll}6 & -5.17842314 & 0.11417729 & -0.26949555\end{array}$

$\begin{array}{lllll}1 & -4.99709448 & 1.04867849 & 0.26550393\end{array}$

$1 \quad-5.143066240 .35927569-1.33502556$

$\begin{array}{lllll}6 & -4.07428691 & -0.89010356 & 0.04670857\end{array}$

$1-4.31464394-1.83683843-0.44541038$

$\begin{array}{lllll}1 & -4.07572028 & -1.09857958 & 1.12175754\end{array}$

$6-2.67385193-0.45408472-0.37964113$

$1-2.67479641-0.23481738-1.45278845$

$1-1.99267976-1.29615568-0.24094531$

$\begin{array}{lllll}6 & -2.13906493 & 0.75946433 & 0.37615159\end{array}$

$\begin{array}{lllll}1 & -2.13669452 & 0.53943749 & 1.44909770\end{array}$

$\begin{array}{lllll}1 & -2.82066476 & 1.60143751 & 0.23879849\end{array}$

$\begin{array}{llll}6 & -0.73924852 & 1.19638335 & -0.05151563\end{array}$

$\begin{array}{lllll}1 & -0.49773285 & 2.14056969 & 0.44460270\end{array}$

$\begin{array}{lllll}1 & -0.74079727 & 1.40972559-1.12544179\end{array}$

$\begin{array}{lllll}6 & 0.36335926 & 0.18918165 & 0.25765899\end{array}$

$1 \quad 0.18446907-0.74208647-0.28609408$

$\begin{array}{lllll}1 & 0.32826247 & -0.06688361 & 1.32182737\end{array}$

$\begin{array}{llll}6 & 1.75454710 & 0.70334402 & -0.09116380\end{array}$

$\begin{array}{lllll}1 & 1.94694734 & 1.62934643 & 0.46010558\end{array}$

$\begin{array}{lllll}1 & 1.78482787 & 0.97059527 & -1.15237576\end{array}$

$\begin{array}{lllll}6 & 2.86248381 & -0.29886210 & 0.20665593\end{array}$

$12.67178982-1.22359019-0.34736790$

$12.83013677-0.568626911 .26715660$

$\begin{array}{lllll}6 & 4.25366769 & 0.21650183 & -0.13890500\end{array}$

$\begin{array}{lllll}1 & 4.44543228 & 1.14085241 & 0.41562111\end{array}$

$1 \quad 4.286315820 .48724937-1.19925943$

$\begin{array}{llll}6 & 5.36272936 & -0.78579812 & 0.15750215\end{array}$

$15.17021664-1.70819519-0.39742410$

$15.32883504-1.055470751 .21672820$

$6 \quad 6.74878659-0.25958413-0.19145075$

$1 \quad 6.976813080 .645606130 .37432076$

$1 \quad 6.81642347-0.01065554-1.25207917$

$17.52343948-0.994357680 .02861835$ 
energy $\quad 1.05279$

Symmetry point group: C1

moment of inertia 2.1065

Cartesian coordinate of structure $\quad 18$

$6 \quad 5.21296952-1.95579391-0.47579621$

$1 \quad 6.23298899-1.69571436-0.75925097$

$1 \quad 5.26643023-2.665234150 .35206851$

$14.75285248-2.47075058-1.32122670$

$6 \quad 4.40782731-0.72256237-0.08690009$

$14.40644728-0.01674282-0.92027065$

$14.89764724-0.211193030 .74703965$

$6 \quad 2.97351197-1.059565330 .30884320$

$12.99664270-1.823738931 .09104302$

$12.46430773-1.51618321-0.54666574$

$\begin{array}{lllll}6 & 2.15464457 & 0.12975506 & 0.80330035\end{array}$

$1 \quad 2.641578940 .564578761 .68223023$

$1 \quad 1.18315783-0.230583771 .14597936$

$\begin{array}{lllll}6 & 1.95239237 & 1.22952181 & -0.23875176\end{array}$

$1 \quad 1.612241790 .77961260-1.17799744$

$12.918756111 .68597354-0.45915583$

$\begin{array}{lllll}6 & 0.96267070 & 2.32229957 & 0.19019845\end{array}$

$1 \quad 1.309260093 .29023548-0.17664892$

$\begin{array}{llll}1 & 0.96130892 & 2.39724172 & 1.28222076\end{array}$

$\begin{array}{llll}6 & -0.46979609 & 2.12167303 & -0.30498363\end{array}$

$1-0.471002222 .14617564-1.39963853$

$\begin{array}{lllll}1 & -1.07479350 & 2.97414341 & 0.01729680\end{array}$

$\begin{array}{lllll}6 & -1.14831143 & 0.83738947 & 0.15717354\end{array}$

$\begin{array}{lllll}1 & -1.10387041 & 0.77404995 & 1.24938630\end{array}$

$1-0.59929664-0.02918393-0.22219000$

$\begin{array}{llll}6 & -2.59955808 & 0.73636232 & -0.29539461\end{array}$

$1-2.64325706 \quad 0.81323745-1.38663659$

$\begin{array}{lllll}1 & -3.16002001 & 1.59338720 & 0.09160570\end{array}$

$\begin{array}{llll}6 & -3.28143718 & -0.55227158 & 0.14581293\end{array}$

$1-3.23543800-0.630890551 .23688975$

$1-2.72206501-1.40926832-0.24331514$

$6-4.73386105-0.65492150-0.30400880$

$1-4.77822075-0.57566012-1.39376785$

$\begin{array}{lllll}1 & -5.29151954 & 0.20102315 & 0.08609793\end{array}$

$\begin{array}{lllll}6 & -5.40454552 & -1.94767515 & 0.14181459\end{array}$

$\begin{array}{lllll}1 & -5.39914426 & -2.03544317 & 1.22980988\end{array}$

$1-4.88272686-2.81757120-0.26124320$

$1-6.44130866-1.99676935-0.19150813$ energy $\quad 2.60611$

Symmetry point group: C1 moment of inertia 2.1364

Cartesian coordinate of structure 19

$65.49228176-1.78400352-0.21059357$ 
$1 \quad 6.47372579-1.49672172-0.58827831$

$15.63531955-2.271336620 .75562400$

$15.07557275-2.52554800-0.89473708$

$64.56708415-0.58047887-0.08354623$

$14.47567584-0.09399567-1.05684050$

$\begin{array}{lllll}1 & 5.01498590 & 0.15821111 & 0.58753489\end{array}$

$\begin{array}{llll}6 & 3.18538588 & -0.95658205 & 0.44259649\end{array}$

$13.30829290-1.499786161 .38390030$

$12.71501328-1.65912110-0.25351981$

$\begin{array}{lllll}6 & 2.24725059 & 0.22628414 & 0.67362417\end{array}$

$\begin{array}{lllll}1 & 2.72167114 & 0.93719041 & 1.35867030\end{array}$

$1 \quad 1.35288204-0.137361631 .18513194$

$\begin{array}{llll}6 & 1.83533428 & 0.95544906 & -0.60150618\end{array}$

$1 \quad 1.350818690 .24410601-1.27685762$

$1 \quad 2.725321951 .30817191-1.12660112$

$\begin{array}{llll}6 & 0.91835823 & 2.15645196-0.36562724\end{array}$

$10.685653642 .61124605-1.33259561$

$\begin{array}{lllll}1 & 1.46728497 & 2.91237823 & 0.20295764\end{array}$

$\begin{array}{lllll}6 & -0.39130185 & 1.85240694 & 0.36203247\end{array}$

$\begin{array}{lllll}1 & -0.96249259 & 2.78082091 & 0.44840832\end{array}$

$\begin{array}{lllll}1 & -0.18355439 & 1.53475256 & 1.38757987\end{array}$

$\begin{array}{lllll}6 & -1.25850853 & 0.80077423 & -0.31984756\end{array}$

$1-0.73090532-0.15722163-0.34310654$

$\begin{array}{lllll}1 & -1.41857784 & 1.08465201 & -1.36535479\end{array}$

$\begin{array}{lllll}6 & -2.60593212 & 0.60446304 & 0.36327775\end{array}$

$\begin{array}{llll}1 & -3.14811096 & 1.55537952 & 0.37455393\end{array}$

$\begin{array}{lllll}1 & -2.44225960 & 0.33653125 & 1.41215754\end{array}$

$6-3.47088317-0.46052344-0.29835135$

$1-2.93010711-1.41249060-0.30615471$

$1-3.63212214-0.19523106-1.34828905$

$\begin{array}{llll}6 & -4.82001320 & -0.65533500 & 0.38308604\end{array}$

$\begin{array}{lllll}1 & -5.35962161 & 0.29576533 & 0.38883837\end{array}$

$1-4.65726246-0.918964441 .43182601$

$6-5.67524543-1.72375191-0.28538083$

$1-5.16946208-2.69100388-0.27633885$

$1-5.87693329-1.46742500-1.32705673$

$1-6.63367226-1.843925240 .21996394$ energy $\quad 1.06712$

Symmetry point group: C1 moment of inertia 2.1904

Cartesian coordinate of structure 20

$\begin{array}{lllll}6 & -4.88453078 & 2.26202056 & -0.14283854\end{array}$

$1-5.007313752 .90581331-1.01398560$

$\begin{array}{lllll}1 & -5.86641029 & 2.12697914 & 0.31438605\end{array}$

$\begin{array}{lllll}1 & -4.25646703 & 2.79066157 & 0.57674482\end{array}$

$\begin{array}{lllll}6 & -4.26833653 & 0.92099095 & -0.51950174\end{array}$

$1 \quad-3.30696331 \quad 1.09243575-1.00988209$

$1-4.901040630 .41821774-1.25682495$ 


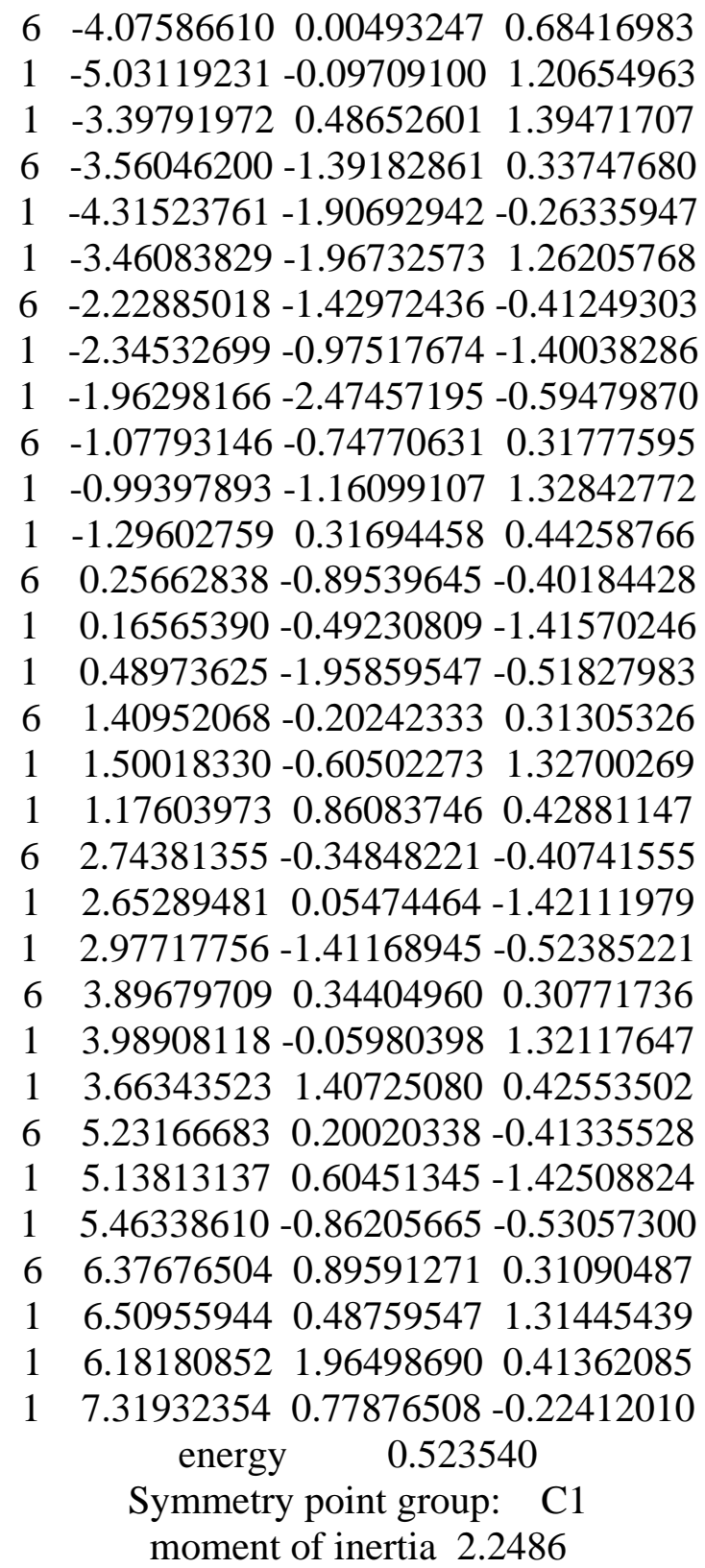

Cartesian coordinate of structure 21

$64.168872752 .49997861-0.55213180$

$13.783028423 .49487115-0.32912848$

$15.241267922 .50365690-0.34880804$

$14.037370252 .32319689-1.62133465$

$\begin{array}{lllll}6 & 3.46119197 & 1.42809326 & 0.26648416\end{array}$

$\begin{array}{lllll}1 & 2.38718869 & 1.47721226 & 0.07062469\end{array}$

13.586152831 .635833891 .33325575

$\begin{array}{lllll}6 & 3.97585018 & 0.02469192 & -0.03511445\end{array}$

$1 \quad 5.061721180 .011232140 .09372005$

$13.79836282-0.20120727-1.09050031$

$6 \quad 3.36864454-1.071255670 .84107599$

$13.67608576-0.904736981 .87729808$

$1 \quad 3.79627271-2.033386890 .54531665$ 


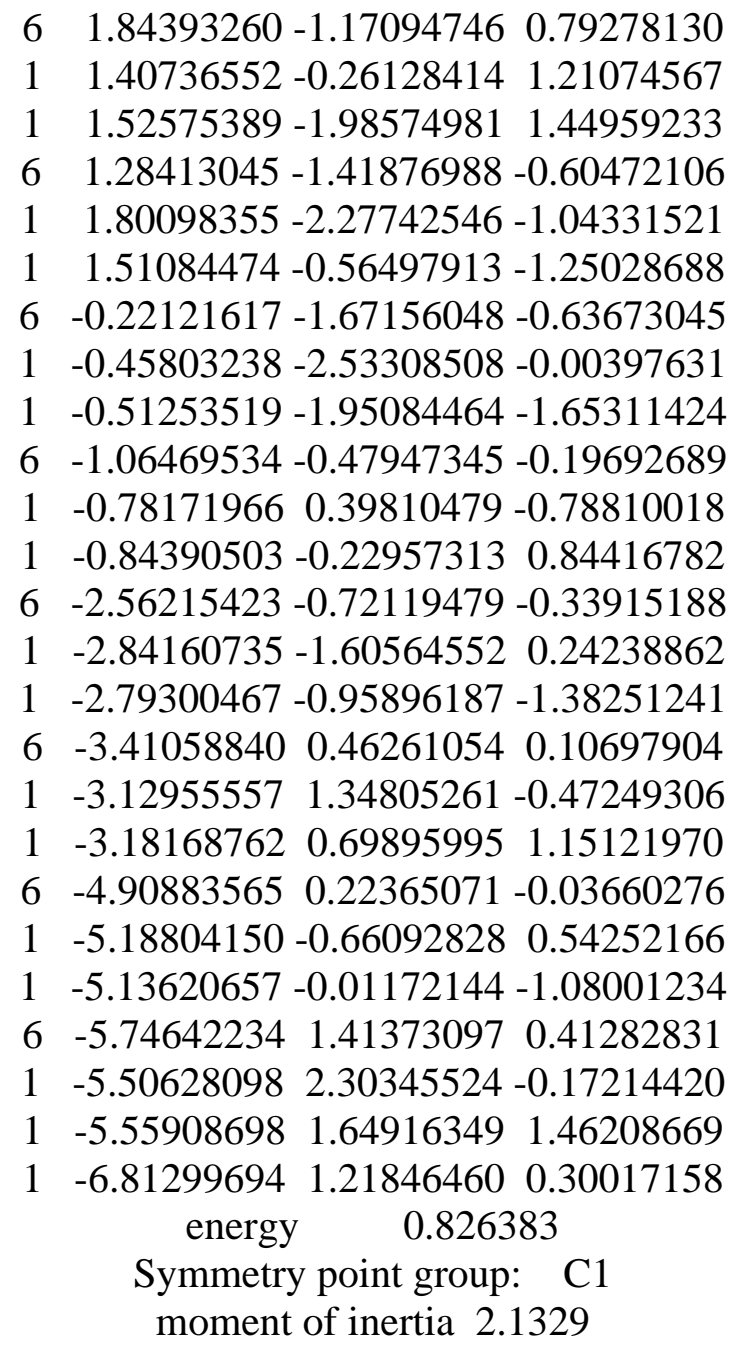

Cartesian coordinate of structure 22

$65.73229665-1.16251219-0.34726607$

$15.97217274-1.90729295-1.10633420$

$16.59035435-0.49482565-0.25070234$

$15.61185303-1.683212360 .60461564$

$64.47235960-0.38452199-0.70406048$

$13.64285678-1.08398728-0.83354694$

$14.606076670 .11160434-1.66985188$

$\begin{array}{lllll}6 & 4.10794361 & 0.65670796 & 0.34851957\end{array}$

$\begin{array}{lllll}1 & 4.97440519 & 1.30077205 & 0.52289520\end{array}$

$\begin{array}{lllll}1 & 3.91742084 & 0.15148223 & 1.29967029\end{array}$

$\begin{array}{llll}6 & 2.91612374 & 1.53886718-0.02353573\end{array}$

$13.183950752 .14052668-0.89664310$

$\begin{array}{llll}1 & 2.73641222 & 2.24628770 & 0.79090163\end{array}$

$\begin{array}{llll}6 & 1.61428888 & 0.79545294 & -0.32601403\end{array}$

$1 \quad 1.739388330 .17207284-1.21634845$

$1 \quad 0.854566581 .53613993-0.58352914$

$\begin{array}{lllll}6 & 1.11278623 & -0.07441854 & 0.82232397\end{array}$

$1 \quad 0.98920096 \quad 0.54566272 \quad 1.71670040$

$1 \quad 1.87440932-0.817365511 .07065867$ 


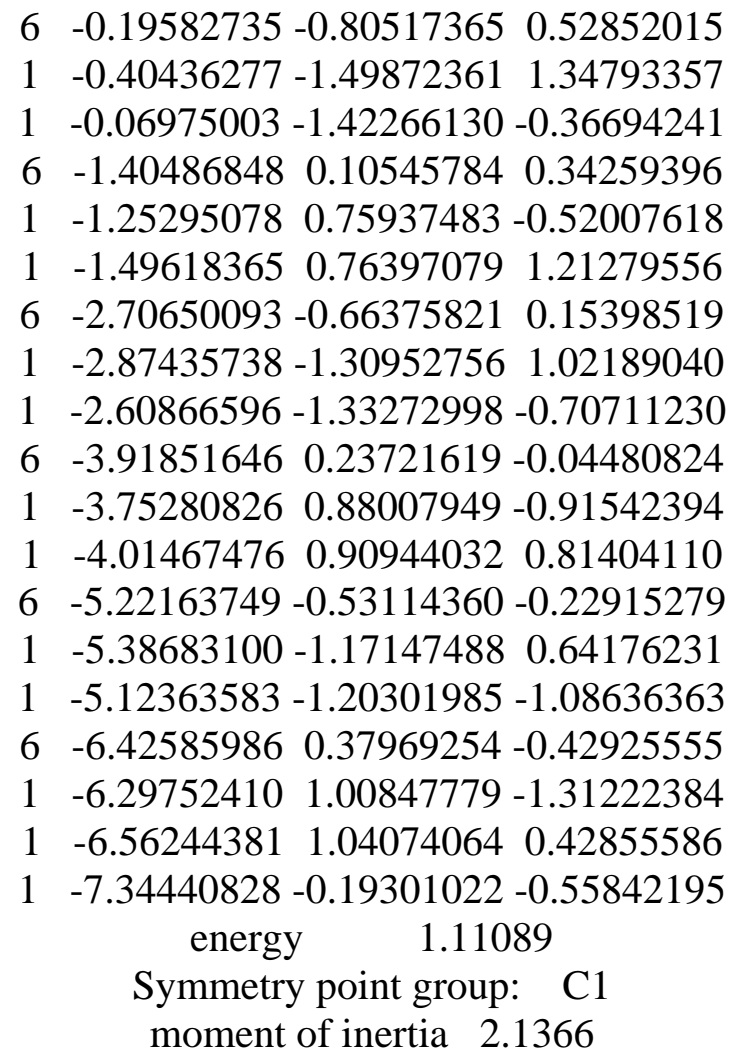

Cartesian coordinate of structure 23

$\begin{array}{llll}6 & -4.62488014 & 2.27006425 & -0.27666881\end{array}$

$\begin{array}{llll}1 & -5.35660980 & 2.49472678 & -1.05287874\end{array}$

$\begin{array}{lllll}1 & -5.03739547 & 2.60146673 & 0.67802105\end{array}$

$1 \quad-3.733590932 .86828197-0.47542711$

$\begin{array}{llll}6 & -4.28539325 & 0.78575179 & -0.23943113\end{array}$

$1-3.909806580 .47823408-1.21825143$

$\begin{array}{llll}1 & -5.19492873 & 0.20346326 & -0.06529370\end{array}$

$\begin{array}{lllll}6 & -3.26013547 & 0.44515917 & 0.83674849\end{array}$

$\begin{array}{lllll}1 & -3.62518046 & 0.81157720 & 1.80024857\end{array}$

$\begin{array}{lllll}1 & -2.33719934 & 0.99879671 & 0.63808345\end{array}$

$\begin{array}{llll}6 & -2.95372809 & -1.04603348 & 0.96648193\end{array}$

$1-3.86551536-1.570121821 .26603924$

$1-2.24360205-1.188623011 .78427805$

$6-2.40951018-1.71038284-0.30106982$

$1-3.19459957-1.74232035-1.06006457$

$1-2.17350735-2.75268980-0.06951776$

6 - $1.17231427-1.04475012-0.90180528$

$1-1.42030894-0.03266850-1.23588195$

$1-0.88413696-1.59348140-1.80257732$

$\begin{array}{lllll}6 & 0.02453058 & -0.97895202 & 0.03977112\end{array}$

$1 \quad 0.24973995-1.984982580 .40907045$

$\begin{array}{llll}1 & -0.22755597 & -0.38175242 & 0.92072115\end{array}$

$6 \quad 1.26747521-0.39028600-0.61530298$

$1 \quad 1.036276570 .61185041-0.99075959$

$1 \quad 1.53266510-0.98882708-1.49265148$ 


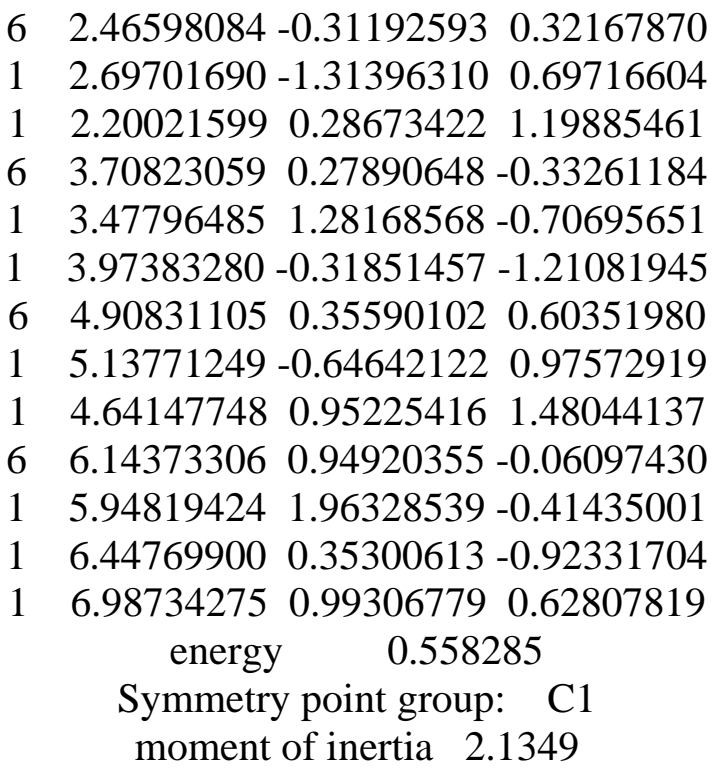

Cartesian coordinate of structure 24

$65.51492901-0.77148394-0.77198903$

$16.46255338-0.24176475-0.67301494$

$15.70559527-1.83273001-0.60220248$

$15.17607581-0.65901510-1.80361192$

$\begin{array}{lllll}6 & 4.47197065 & -0.24117876 & 0.20315282\end{array}$

14.332510490 .829094530 .03341571

$1 \quad 4.84047627-0.341888291 .22820131$

$\begin{array}{llll}6 & 3.13362684 & -0.96153020 & 0.07927297\end{array}$

$13.30093750-2.037017370 .18535397$

$1 \quad 2.74474291-0.81862447-0.93345969$

$\begin{array}{lllll}6 & 2.08708214 & -0.52753103 & 1.10467718\end{array}$

$12.45103136-0.771772752 .10648067$

$1 \quad 1.18420240-1.126372450 .95938197$

$\begin{array}{lllll}6 & 1.72101325 & 0.95763011 & 1.07171413\end{array}$

$1 \quad 2.583178341 .55287504 \quad 1.38084684$

$\begin{array}{lllll}1 & 0.95188101 & 1.14090216 & 1.82537277\end{array}$

$\begin{array}{llll}6 & 1.23865700 & 1.47243642 & -0.28585159\end{array}$

$12.066112941 .44249247-0.99951850$

$1 \quad 0.971357092 .52745883-0.18030497$

$\begin{array}{llll}6 & 0.04989603 & 0.71863013-0.88090712\end{array}$

$10.33369641-0.31665574-1.09200006$

$\begin{array}{llll}1 & -0.19580971 & 1.16395555-1.84885450\end{array}$

$\begin{array}{llll}6 & -1.19749759 & 0.72335694 & -0.00488946\end{array}$

$\begin{array}{lllll}1 & -1.45670380 & 1.75624111 & 0.25035617\end{array}$

$\begin{array}{lllll}1 & -0.98793626 & 0.21989778 & 0.94320210\end{array}$

$\begin{array}{lllll}6 & -2.39361706 & 0.05033516 & -0.66600757\end{array}$

$1-2.12704198-0.97782622-0.93157292$

$1-2.619366160 .55814526-1.60915798$

$\begin{array}{lllll}6 & -3.64007926 & 0.03628676 & 0.20936508\end{array}$

$\begin{array}{lllll}1 & -3.90563126 & 1.06402493 & 0.47760817\end{array}$

$1-3.41490448-0.474006661 .15151058$ 


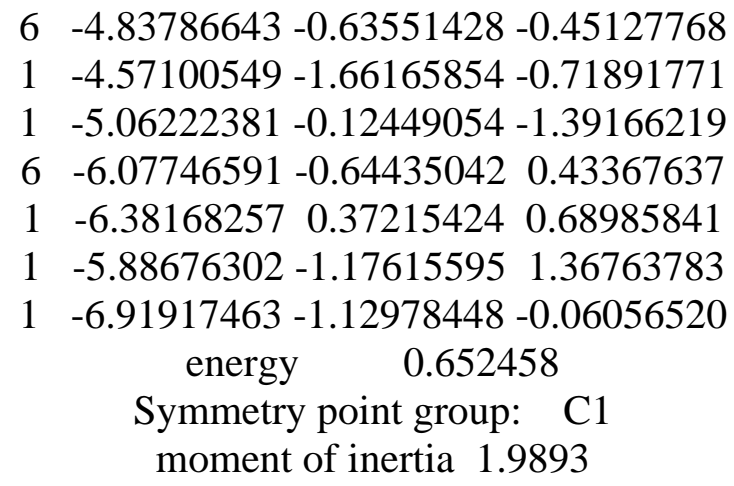

Cartesian coordinate of structure 25

$\begin{array}{llll}6 & -3.91533689 & 2.47011501 & -0.72885020\end{array}$

$\begin{array}{llll}1 & -4.89309601 & 2.57473776-1.19944295\end{array}$

$\begin{array}{lllll}1 & -3.81262695 & 3.26685015 & 0.01011727\end{array}$

$1-3.156414062 .63350689-1.49634306$

$\begin{array}{lllll}6 & -3.74787313 & 1.10106816-0.08262523\end{array}$

$1-3.894568530 .32656296-0.83912312$

$\begin{array}{lllll}1 & -4.53039188 & 0.94831656 & 0.66641404\end{array}$

$\begin{array}{lllll}6 & -2.38252801 & 0.92731404 & 0.57409119\end{array}$

$\begin{array}{lllll}1 & -2.21884034 & 1.75336363 & 1.27199351\end{array}$

$1-1.606970931 .02491365-0.19056557$

$\begin{array}{lllll}6 & -2.20965663 & -0.38913484 & 1.33004874\end{array}$

$1-2.92904615-0.416674792 .15358041$

$\begin{array}{lllll}1 & -1.22276823 & -0.40601701 & 1.79801869\end{array}$

$\begin{array}{llll}6 & -2.39999003 & -1.65424669 & 0.49145901\end{array}$

$\begin{array}{lllll}1 & -3.44172541 & -1.71060784 & 0.16621325\end{array}$

$1-2.24418221-2.521979501 .13860668$

6 -1.50402837 -1.80459477 -0.74020219

$1-1.66995681-0.97082801-1.42914839$

$1-1.83872021-2.69734271-1.27253109$

$6-0.00254934-1.94092543-0.45922230$

$10.44967151-2.54336445-1.25172120$

$1 \quad 0.14135941-2.508782180 .46602634$

$6 \quad 0.77600792-0.63084210-0.37406889$

$1 \quad 0.39281752-0.009443450 .43748551$

$1 \quad 0.61467307-0.06031641-1.29457944$

$6 \quad 2.27084455-0.84162738-0.16972087$

$12.66597613-1.45365809-0.98696225$

$1 \quad 2.43188052-1.418892620 .74651621$

$\begin{array}{lllll}6 & 3.05809892 & 0.45991517 & -0.08850214\end{array}$

$1 \quad 2.66335722 \quad 1.071201750 .72955344$

$1 \quad 2.89637237 \quad 1.03845039-1.00386264$

$\begin{array}{lllll}6 & 4.55384527 & 0.25249317 & 0.11660520\end{array}$

$14.94693694-0.35744645-0.70147140$

$14.71362948-0.325676151 .03095317$

$\begin{array}{lllll}6 & 5.33002696 & 1.56048852 & 0.19724587\end{array}$

14.975048892 .174814661 .02673590

$15.210490532 .14270351-0.71829338$ 
$1 \quad 6.39592670 \quad 1.385470650 .34428096$

energy $\quad 2.22967$

Symmetry point group: C1

moment of inertia 2.1780

Cartesian coordinate of structure 26

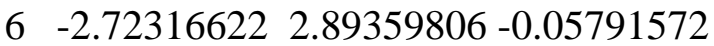

$\begin{array}{lllll}1 & -2.69207978 & 3.55106984 & -0.92684115\end{array}$

$\begin{array}{lllll}1 & -3.38763829 & 3.34562632 & 0.68078099\end{array}$

$\begin{array}{lllll}1 & -1.72095325 & 2.86545491 & 0.37479108\end{array}$

$\begin{array}{lllll}6 & -3.19022255 & 1.49283584 & -0.43070643\end{array}$

$1-2.515248941 .07881269-1.18279243$

$\begin{array}{lllll}1 & -4.17591957 & 1.54342525 & -0.90179738\end{array}$

$\begin{array}{lllll}6 & -3.26207639 & 0.56059666 & 0.77356798\end{array}$

$\begin{array}{lllll}1 & -3.96161640 & 0.98312392 & 1.50043052\end{array}$

$\begin{array}{lllll}1 & -2.29117456 & 0.54041327 & 1.27723091\end{array}$

$\begin{array}{lllll}6 & -3.70449911 & -0.86395706 & 0.44557398\end{array}$

$1-4.70180325-0.82659751-0.00265584$

$1-3.81339189-1.417815541 .38229300$

$6-2.78604881-1.66727075-0.47983672$

$1-2.74757146-1.20212449-1.46869162$

$1-3.25409844-2.64194699-0.63134783$

$\begin{array}{llll}6 & -1.35699024 & -1.87625558 & 0.03796500\end{array}$

$1-0.99207024-2.84946893-0.30044292$

$1-1.37138418-1.927004191 .13181877$

$6-0.34703695-0.82075272-0.40559265$

$1-0.667953610 .17272844-0.08513236$

$1-0.32228320-0.79108515-1.49972371$

$\begin{array}{llll}6 & 1.05781309 & -1.07936615 & 0.12271458\end{array}$

$11.39264831-2.06899268-0.20447585$

$1 \quad 1.03079883-1.114765511 .21661495$

$62.07059064-0.03148040-0.32110803$

$\begin{array}{lllll}1 & 1.73358832 & 0.95730056 & 0.00659396\end{array}$

$1 \quad 2.09741180 \quad 0.00471881-1.41490599$

$\begin{array}{llll}6 & 3.47616149 & -0.28453208 & 0.20851382\end{array}$

$13.81449491-1.27259891-0.12003196$

$1 \quad 3.44953270-0.321838601 .30241259$

$6 \quad 4.48861580 \quad 0.76531001-0.23336584$

$\begin{array}{lllll}1 & 4.14943021 & 1.75145028 & 0.09574622\end{array}$

$14.51407997 \quad 0.80152265-1.32600692$

$\begin{array}{lllll}6 & 5.89012552 & 0.50231693 & 0.30179817\end{array}$

$1 \quad 6.26303535-0.46533412-0.03885414$

$\begin{array}{lllll}1 & 5.89584947 & 0.49166273 & 1.39327082\end{array}$

$1 \quad 6.59471970 \quad 1.26600629-0.02793258$ energy $\quad 1.86786$

Symmetry point group: C1

moment of inertia 2.1270

Cartesian coordinate of structure 
$\begin{array}{llll}6 & 0.22204385 & 2.93754173-0.01672483\end{array}$

$\begin{array}{lllll}1 & -0.14157229 & 3.58783463 & 0.77898125\end{array}$

$10.408338263 .55613012-0.89655228$

$\begin{array}{lllll}1 & -0.57852489 & 2.23839316 & -0.26947615\end{array}$

$\begin{array}{lllll}6 & 1.47855762 & 2.18484187 & 0.40135261\end{array}$

$\begin{array}{lllll}1 & 1.25579736 & 1.59064413 & 1.29052422\end{array}$

$\begin{array}{llll}1 & 2.25748409 & 2.89256652 & 0.69814237\end{array}$

$\begin{array}{llll}6 & 2.00975041 & 1.28698075 & -0.71131089\end{array}$

$12.315331041 .92058579-1.54880061$

$1 \quad 1.189367530 .67472771-1.08853947$

$\begin{array}{lllll}6 & 3.19845766 & 0.40749290 & -0.31152770\end{array}$

$\begin{array}{lllll}1 & 3.98996488 & 1.06759807 & 0.05434554\end{array}$

$13.60108258-0.07409390-1.20754207$

$6 \quad 2.93556756-0.67037804 \quad 0.74857361$

$1 \quad 2.29557753-0.270382711 .53766323$

$13.88340826-0.903122911 .23860153$

$\begin{array}{llll}6 & 2.36177203 & -1.99484011 & 0.23362807\end{array}$

$1 \quad 2.26898742-2.676692551 .08413864$

$13.09345663-2.44937172-0.44035954$

$6 \quad 1.01716725-1.92689452-0.48987174$

$1 \quad 0.73389507-2.93815072-0.79448002$

$1 \quad 1.13004720-1.36259572-1.41894384$

$\begin{array}{llll}6 & -0.11477326 & -1.32392729 & 0.33407445\end{array}$

$1 \quad 0.19639900-0.36228800 \quad 0.74838088$

$\begin{array}{llll}1 & -0.32266417 & -1.96855112 & 1.19387633\end{array}$

$6-1.39095309-1.10663626-0.46766710$

$1-1.73705034-2.06020070-0.87870877$

$1-1.16363639-0.46947564-1.32948856$

$\begin{array}{lllll}6 & -2.50803256 & -0.46503378 & 0.34461986\end{array}$

$\begin{array}{lllll}1 & -2.14725642 & 0.47860988 & 0.76789368\end{array}$

$1-2.75135568-1.105693651 .19831420$

$6-3.77213603-0.19978812-0.46361674$

$1-4.13363991-1.14174575-0.88524175$

$1 \quad-3.524351960 .43973182-1.31543997$

$\begin{array}{lllll}6 & -4.87715740 & 0.45050079 & 0.35838758\end{array}$

$\begin{array}{lllll}1 & -4.54831814 & 1.40868906 & 0.76486333\end{array}$

$1-5.16298100-0.183504441 .19968341$

$1-5.769369850 .63119912-0.24133868$ energy $\quad 1.50802$

Symmetry point group: C1

moment of inertia 1.9906

Cartesian coordinate of structure 28

$64.552136162 .43621940-0.27331746$

14.445113193 .331034620 .34009092

$15.608614152 .32910953-0.52673632$

$14.006055612 .60221661-1.20389070$

$\begin{array}{lllll}6 & 4.03538605 & 1.19830769 & 0.44739494\end{array}$

$\begin{array}{lllll}1 & 2.98531592 & 1.33975030 & 0.70926008\end{array}$ 
14.568784241 .075277651 .39398629

$6 \quad 4.20214063-0.07598579-0.37747089$

$15.26796967-0.20733636-0.58104870$

$13.727164750 .06039065-1.35490182$

$\begin{array}{llll}6 & 3.64695327 & -1.34368352 & 0.28636115\end{array}$

$13.69416514-1.229832891 .37402646$

$14.29329356-2.189697100 .04500813$

$6 \quad 2.22122540-1.71593876-0.12093175$

$1 \quad 1.95004818-2.653201270 .37367933$

$12.20354161-1.92470770-1.19558566$

$\begin{array}{lllll}6 & 1.15688396 & -0.67119877 & 0.19375435\end{array}$

$1 \quad 1.360763090 .24558740-0.36653659$

$1 \quad 1.21262182-0.401459571 .25349638$

$6-0.25422332-1.14290465-0.13392141$

$1-0.47291973-2.052806310 .43400520$

$1-0.30397631-1.42560317-1.19042431$

$\begin{array}{lllll}6 & -1.32314788 & -0.09761760 & 0.15816083\end{array}$

$\begin{array}{lllll}1 & -1.10443198 & 0.81146565 & -0.41118679\end{array}$

$\begin{array}{lllll}1 & -1.27163609 & 0.18638000 & 1.21414658\end{array}$

$6-2.73507052-0.56746882-0.16802530$

$1-2.95404660-1.476544090 .40121536$

$1-2.78638247-0.85127888-1.22408200$

$\begin{array}{lllll}6 & -3.80332380 & 0.47840200 & 0.12418512\end{array}$

$1-3.584166341 .38819435-0.44411798$

$\begin{array}{lllll}1 & -3.75309662 & 0.76182835 & 1.18052720\end{array}$

$\begin{array}{lllll}6 & -5.21618359 & 0.01033536 & -0.20324437\end{array}$

$\begin{array}{lllll}1 & -5.43372297 & -0.89842337 & 0.36478071\end{array}$

$1-5.26498829-0.27173965-1.25864352$

$\begin{array}{lllll}6 & -6.27534449 & 1.06389123 & 0.09344038\end{array}$

$1-6.096076061 .97174550-0.48546979$

$\begin{array}{lllll}1 & -6.26670049 & 1.33982437 & 1.14949627\end{array}$

$\begin{array}{lllll}1 & -7.27589842 & 0.70567881 & -0.14940829\end{array}$ energy $\quad 2.11875$

Symmetry point group: C1 moment of inertia 2.1279

Cartesian coordinate of structure 29

$65.49298557 \quad 1.35911540-0.66771046$

$15.610580202 .37305986-0.28479601$

$1 \quad 6.423504290 .82111403-0.47732755$

$15.36486396 \quad 1.42387583-1.74977471$

$6 \quad 4.308813320 .65151319-0.02180611$

$13.399054251 .22332070-0.21876807$

$\begin{array}{lllll}1 & 4.43453180 & 0.63702527 & 1.06462978\end{array}$

$64.13689938-0.77667389-0.52832920$

$15.07383177-1.31841683-0.36907873$

$13.98337701-0.74871365-1.61223810$

$6 \quad 2.99367809-1.566565490 .10925065$

$13.17869044-1.674223421 .18265219$ 
$13.01827442-2.57710003-0.30281486$

$61.59884826-0.96187091-0.10577460$

$1 \quad 0.87671248-1.76535967-0.25955361$

$11.59594734-0.37699786-1.03234849$

$6 \quad 1.11194421-0.084613991 .04734468$

$\begin{array}{llll}1 & 1.89711768 & 0.61781703 & 1.33251377\end{array}$

$\begin{array}{llll}1 & 0.94281412 & -0.71900008 & 1.92333941\end{array}$

$\begin{array}{lllll}6 & -0.16075910 & 0.69966591 & 0.73868240\end{array}$

$1 \quad 0.036685731 .39659888-0.08262749$

$\begin{array}{lllll}1 & -0.41490845 & 1.31673412 & 1.60524754\end{array}$

$\begin{array}{lllll}6 & -1.36571860 & -0.16434277 & 0.38109505\end{array}$

$\begin{array}{lllll}1 & -1.50101886 & -0.93081629 & 1.15185777\end{array}$

$1-1.17555947-0.70061898-0.55242733$

$\begin{array}{lllll}6 & -2.65348591 & 0.63695265 & 0.23735128\end{array}$

$\begin{array}{llll}1 & -2.51252358 & 1.41601353 & -0.51893141\end{array}$

$\begin{array}{lllll}1 & -2.85926338 & 1.15943414 & 1.17704256\end{array}$

$6-3.85836149-0.21622569-0.13798069$

$1-3.98894037-1.004885880 .61026443$

$1-3.66050808-0.72676644-1.08600008$

$\begin{array}{lllll}6 & -5.15225691 & 0.58017357 & -0.25734424\end{array}$

$\begin{array}{llll}1 & -5.02184920 & 1.36711668 & -1.00545667\end{array}$

$\begin{array}{lllll}1 & -5.34753060 & 1.08949844 & 0.69034244\end{array}$

$6-6.34975076-0.28436959-0.62955289$

$1-6.51630803-1.062070950 .11812542$

$1-6.19121718-0.77870651-1.58971922$

$\begin{array}{llll}1 & -7.26337460 & 0.30551780 & -0.70550817\end{array}$ energy $\quad 2.56705$

Symmetry point group: C1 moment of inertia 2.0798

Cartesian coordinate of structure $\quad 30$

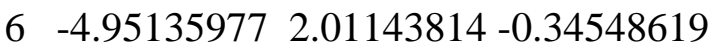

$\begin{array}{llll}1 & -5.41939746 & 2.28314212 & -1.29182299\end{array}$

$\begin{array}{lllll}1 & -5.70339914 & 2.10999855 & 0.43972295\end{array}$

$\begin{array}{lllll}1 & -4.16486135 & 2.74028962 & -0.14073708\end{array}$

$\begin{array}{lllll}6 & -4.38621796 & 0.59792276 & -0.38593383\end{array}$

$1-3.655368290 .51995080-1.19265499$

$1-5.18357888-0.10910116-0.63096374$

$\begin{array}{lllll}6 & -3.75141752 & 0.18024806 & 0.93865114\end{array}$

$\begin{array}{lllll}1 & -4.52212753 & 0.23506108 & 1.71181523\end{array}$

$\begin{array}{lllll}1 & -2.99117094 & 0.91534023 & 1.22366997\end{array}$

$\begin{array}{llll}6 & -3.12748209 & -1.22225755 & 0.93687523\end{array}$

$1-3.66112106-1.852867150 .21861891$

$\begin{array}{llll}1 & -3.28025087 & -1.68432150 & 1.91410235\end{array}$

$\begin{array}{lllll}6 & -1.62987397 & -1.26271787 & 0.63204747\end{array}$

$\begin{array}{lllll}1 & -1.28184660 & -2.29659574 & 0.71992638\end{array}$

$\begin{array}{lllll}1 & -1.10399952 & -0.69797079 & 1.40672657\end{array}$

$6-1.23448699-0.73198721-0.74291881$

$\begin{array}{lllll}1 & -1.45387596 & 0.33801308 & -0.80223636\end{array}$ 
$1-1.85699031-1.21572438-1.50108323$

$6 \quad 0.23558358-0.95178599-1.09290172$

$1 \quad 0.40364049-0.63864774-2.12714534$

$1 \quad 0.45915571-2.02304571-1.05867029$

$61.21448581-0.20685529-0.19151176$

$\begin{array}{lllll}1 & 1.11931232 & -0.56291754 & 0.83768991\end{array}$

$1 \quad 0.948874530 .85551075-0.17274928$

$6 \quad 2.66490171-0.35589202-0.63335212$

$\begin{array}{llll}1 & 2.77047447 & 0.01280642 & -1.65871685\end{array}$

$12.92503178-1.41884768-0.66511226$

$\begin{array}{lllll}6 & 3.65024738 & 0.37612621 & 0.26860082\end{array}$

$\begin{array}{lllll}1 & 3.54789950 & 0.00459924 & 1.29338703\end{array}$

$\begin{array}{lllll}1 & 3.38803644 & 1.43855679 & 0.30405298\end{array}$

$\begin{array}{llll}6 & 5.10109620 & 0.23184983-0.17491773\end{array}$

$15.202096020 .60425233-1.19811194$

$15.36137912-0.82966125-0.21053025$

$\begin{array}{lllll}6 & 6.07687699 & 0.96681805 & 0.73475325\end{array}$

$\begin{array}{llll}1 & 6.01614158 & 0.59080432 & 1.75768546\end{array}$

$\begin{array}{lllll}1 & 5.85532625 & 2.03526825 & 0.76305514\end{array}$

$\begin{array}{lllll}1 & 7.10649936 & 0.84866436 & 0.39664717\end{array}$ energy 2.56653

Symmetry point group: C1 moment of inertia 2.0741

Cartesian coordinate of structure 31

$\begin{array}{llll}6 & -3.17349625 & 2.85753595 & 0.38494769\end{array}$

$\begin{array}{lllll}1 & -2.41646179 & 3.63815500 & 0.30783897\end{array}$

$\begin{array}{llll}1 & -4.08073666 & 3.22225249 & -0.10042506\end{array}$

$1-3.400583162 .716289461 .44330588$

$\begin{array}{lllll}6 & -2.70089134 & 1.55562433 & -0.24835067\end{array}$

$\begin{array}{lllll}1 & -1.77781922 & 1.23335379 & 0.23606573\end{array}$

$1-2.447323441 .72904704-1.29796772$

$\begin{array}{llll}6 & -3.75405567 & 0.45176453 & -0.17306127\end{array}$

$1-4.660214260 .82502522-0.65755962$

$\begin{array}{lllll}1 & -4.02513027 & 0.28151765 & 0.87437793\end{array}$

$6-3.35118305-0.88072714-0.81833247$

$1-2.69365681-0.68543105-1.66993718$

$1-4.24108339-1.35500635-1.23679753$

$\begin{array}{lllll}6 & -2.70095298 & -1.89409891 & 0.12782671\end{array}$

$1-2.50573262-2.81446829-0.43043359$

$1 \quad-3.42954939-2.156095560 .90020172$

$\begin{array}{lllll}6 & -1.40835558 & -1.45167483 & 0.81044795\end{array}$

$\begin{array}{llll}1 & -1.09995615 & -2.23286697 & 1.51078202\end{array}$

$1-1.59879683-0.565538991 .42281415$

$6-0.25564760-1.17059924-0.14591978$

$1-0.55071532-0.41237419-0.87609363$

$1-0.03676026-2.07588161-0.72138386$

$\begin{array}{llll}6 & 1.00688364 & -0.70168839 & 0.56531836\end{array}$

$1 \quad 1.31176113-1.453852091 .29993498$ 
$\begin{array}{lllll}1 & 0.78036633 & 0.20489136 & 1.13646495\end{array}$

$62.16653998-0.42123875-0.38160845$

$1 \quad 1.859078590 .33089271-1.11526945$

$12.39394528-1.32697856-0.95284219$

$\begin{array}{lllll}6 & 3.42679826 & 0.05507959 & 0.32910133\end{array}$

$13.73495111-0.696879651 .06279002$

$\begin{array}{lllll}1 & 3.19941077 & 0.96066799 & 0.90088443\end{array}$

$\begin{array}{lllll}6 & 4.58705054 & 0.33751316-0.61773452\end{array}$

$14.277813051 .08875656-1.34974027$

$14.81318903-0.56773058-1.18801444$

$\begin{array}{lllll}6 & 5.84120733 & 0.81322603 & 0.10379414\end{array}$

$\begin{array}{lllll}1 & 6.18719745 & 0.06559743 & 0.81989476\end{array}$

$1 \quad 5.64795254 \quad 1.73421340 \quad 0.65687431$

$1 \quad 6.655470551 .00814572-0.59433934$ energy $\quad 1.86922$

Symmetry point group: C1 moment of inertia 2.1275

Cartesian coordinate of structure 32

$62.663744152 .97659345-0.73654112$

$1 \quad 1.928066513 .53430055-1.31633692$

$\begin{array}{lllll}1 & 3.01094784 & 3.62202961 & 0.07257660\end{array}$

$13.517721192 .77521539-1.38604209$

$6 \quad 2.078369301 .68143216-0.18909604$

$1 \quad 1.70782706 \quad 1.07486427-1.01550564$

$\begin{array}{lllll}1 & 1.20713105 & 1.90754836 & 0.43175635\end{array}$

$\begin{array}{lllll}6 & 3.08882865 & 0.89352017 & 0.64010043\end{array}$

$\begin{array}{llll}1 & 3.45147279 & 1.55551758 & 1.43159711\end{array}$

$\begin{array}{lllll}1 & 3.96292553 & 0.66484383 & 0.02104597\end{array}$

$\begin{array}{lllll}6 & 2.57705533 & -0.40156547 & 1.28249778\end{array}$

$1 \quad 1.55212843-0.261175621 .63386847$

$1 \quad 3.16966874-0.587549572 .18113559$

$\begin{array}{llll}6 & 2.68397253 & -1.66359728 & 0.42133921\end{array}$

$12.49844726-2.529781151 .06376176$

$1 \quad 3.72503337-1.752449620 .09623847$

$6 \quad 1.78699116-1.80522137-0.81197588$

$12.14421448-2.68171512-1.35737875$

$1 \quad 1.93051537-0.96130331-1.49087458$

$6 \quad 0.29153167-1.99951916-0.53294563$

$1 \quad 0.17249284-2.611370400 .36744302$

$1-0.14249548-2.58429367-1.34871865$

$6-0.53477347-0.72539743-0.38913941$

$1-0.41619219-0.11793602-1.29201045$

$\begin{array}{lllll}1 & -0.15766267 & -0.11503283 & 0.43279169\end{array}$

$6-2.01535454-1.00124975-0.16158029$

$1-2.13501539-1.610933230 .73987123$

$1-2.40405759-1.60384121-0.98891050$

$\begin{array}{lllll}6 & -2.84906196 & 0.26640181 & -0.02576764\end{array}$

$\begin{array}{lllll}1 & -2.72931627 & 0.87645821 & -0.92700579\end{array}$ 
$\begin{array}{llll}1 & -2.45918826 & 0.86880560 & 0.80125891\end{array}$

$\begin{array}{lllll}6 & -4.33109727 & -0.00304850 & 0.20496193\end{array}$

$\begin{array}{lllll}1 & -4.44890310 & -0.61190130 & 1.10565368\end{array}$

$1-4.71923393-0.60457272-0.62166614$

$\begin{array}{lllll}6 & -5.15336957 & 1.27197223 & 0.33909375\end{array}$

$1-5.075928361 .88328029-0.56189045$

$\begin{array}{lllll}1 & -4.80310604 & 1.87618325 & 1.17797937\end{array}$

$\begin{array}{lllll}1 & -6.20850894 & 1.05288358 & 0.50367919\end{array}$

energy

4.44550

Symmetry point group: C1

moment of inertia 2.0369

Cartesian coordinate of structure 33

$\begin{array}{llll}6 & 4.70408386 & -1.89894548 & 0.26702817\end{array}$

$14.56508411-2.787800230 .88266633$

$15.19188798-2.20673410-0.65979783$

$1 \quad 5.38936174-1.232184300 .79377895$

$63.37930491-1.20325267-0.01771148$

$1 \quad 2.90040174-0.941695360 .92657088$

$12.70099190-1.89873535-0.52019533$

$\begin{array}{lllll}6 & 3.54905779 & 0.03784968 & -0.89099508\end{array}$

$14.07447034-0.26827151-1.79985900$

$14.21471008 \quad 0.74645749-0.38667520$

$\begin{array}{llll}6 & 2.25304211 & 0.75509098 & -1.28829028\end{array}$

$1 \quad 1.464534240 .01449562-1.45342923$

$1 \quad 2.40818577 \quad 1.23996820-2.25429970$

$\begin{array}{llll}6 & 1.76532140 & 1.83331369 & -0.31789348\end{array}$

$1 \quad 0.927432502 .35877903-0.78266450$

$12.557261312 .57898844-0.20478563$

$\begin{array}{llll}6 & 1.34413830 & 1.36365098 & 1.07557224\end{array}$

$\begin{array}{llll}1 & 1.00325796 & 2.23689352 & 1.63917068\end{array}$

$\begin{array}{llll}1 & 2.21653510 & 0.98553581 & 1.61346272\end{array}$

$\begin{array}{lllll}6 & 0.24270127 & 0.30493679 & 1.10096360\end{array}$

$\begin{array}{lllll}1 & 0.02683408 & 0.05263497 & 2.14288753\end{array}$

$1 \quad 0.60128745-0.618421200 .63814865$

$\begin{array}{llll}6 & -1.05177929 & 0.72957332 & 0.41711066\end{array}$

$\begin{array}{lllll}1 & -0.86854186 & 0.90753668 & -0.64633959\end{array}$

$\begin{array}{lllll}1 & -1.38593377 & 1.68617522 & 0.83246618\end{array}$

$\begin{array}{lllll}6 & -2.16519651 & -0.30042816 & 0.55901843\end{array}$

$\begin{array}{llll}1 & -2.36693640 & -0.47166673 & 1.62124307\end{array}$

$\begin{array}{llll}1 & -1.82171179 & -1.25933113 & 0.15745136\end{array}$

$\begin{array}{llll}6 & -3.45665560 & 0.10365255 & -0.14021862\end{array}$

$\begin{array}{llll}1 & -3.25535809 & 0.27184329 & -1.20319402\end{array}$

$\begin{array}{lllll}1 & -3.79936899 & 1.06393886 & 0.25869159\end{array}$

$\begin{array}{lllll}6 & -4.57122002 & -0.92581020 & 0.00264688\end{array}$

$1-4.77218665-1.091845151 .06464594$

$1-4.22694373-1.88448423-0.39516379$

$6-5.85668661-0.51239484-0.70195491$

$1-5.68772184-0.36987933-1.77088053$ 


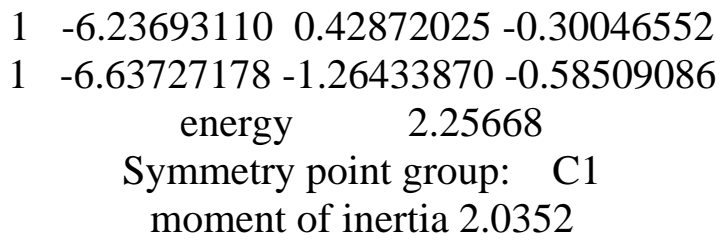

Cartesian coordinate of structure $\quad 34$

$63.915336152 .47011559-0.72884989$

$14.893095352 .57473877-1.19944236$

$13.156413462 .63350720-1.49634294$

13.812625673 .266850640 .01011760

$6 \quad 3.747872761 .10106864-0.08262502$

$\begin{array}{lllll}1 & 4.53039139 & 0.94831728 & 0.66641443\end{array}$

$13.894568630 .32656352-0.83912292$

$\begin{array}{lllll}6 & 2.38252754 & 0.92731398 & 0.57409105\end{array}$

$1 \quad 1.606970651 .02491326-0.19056593$

$1 \quad 2.218839351 .753363561 .27199328$

$6 \quad 2.20965643-0.389134961 .33004858$

$1 \quad 1.22276794-0.406017401 .79801835$

$1 \quad 2.92904581-0.416674692 .15358037$

$\begin{array}{lllll}6 & 2.39999026 & -1.65424674 & 0.49145885\end{array}$

$1 \quad 2.24418277-2.521979621 .13860649$

$1 \quad 3.44172564-1.710607510 .16621302$

$6 \quad 1.50402852-1.80459505-0.74020225$

$11.83872038-2.69734304-1.27253107$

$11.66995686-0.97082836-1.42914858$

$6 \quad 0.00254952-1.94092572-0.45922214$

$1-0.14135908-2.508781900 .46602686$

$1-0.44967140-2.54336526-1.25172061$

$6-0.77600783-0.63084241-0.37406947$

$1-0.61467348-0.06031753-1.29458061$

$\begin{array}{llll}1 & -0.39281706 & -0.00944298 & 0.43748417\end{array}$

$\begin{array}{lllll}6 & -2.27084434 & -0.84162757 & -0.16972044\end{array}$

$1-2.43187978-1.418891750 .74651740$

$1-2.66597634-1.45365926-0.98696089$

$\begin{array}{lllll}6 & -3.05809873 & 0.45991503 & -0.08850280\end{array}$

$1-2.896372991 .03844903-1.00386422$

$\begin{array}{lllll}1 & -2.66335637 & 1.07120274 & 0.72955162\end{array}$

$\begin{array}{lllll}6 & -4.55384490 & 0.25249325 & 0.11660612\end{array}$

$\begin{array}{llll}1 & -4.71362827 & -0.32567475 & 1.03095507\end{array}$

$1-4.94693724-0.35744759-0.70146925$

$\begin{array}{lllll}6 & -5.33002654 & 1.56048867 & 0.19724557\end{array}$

$\begin{array}{lllll}1 & -5.21049109 & 2.14270227 & -0.71829468\end{array}$

$\begin{array}{lllll}1 & -4.97504768 & 2.17481609 & 1.02673430\end{array}$

$\begin{array}{lllll}1 & -6.39592617 & 1.38547099 & 0.34428201\end{array}$ energy $\quad 2.22967$

Symmetry point group: C1

moment of inertia 2.3759 
Cartesian coordinate of structure 35

$\begin{array}{lllll}6 & 6.15227578 & 1.15999537 & 0.43111804\end{array}$

$\begin{array}{lllll}1 & 7.13318375 & 1.55098862 & 0.16032011\end{array}$

$\begin{array}{lllll}1 & 5.42136134 & 1.94535881 & 0.23704256\end{array}$

$\begin{array}{lllll}1 & 6.15480821 & 0.96784427 & 1.50604582\end{array}$

$65.83390225-0.11243863-0.34647477$

$16.64398423-0.83053489-0.20115257$

$1 \quad 5.812423750 .11089589-1.41699922$

$\begin{array}{lllll}6 & 4.51536062 & -0.77204223 & 0.05303261\end{array}$

$14.54222327-1.012715991 .12093716$

$14.42356622-1.72702796-0.47193689$

$6 \quad 3.276596600 .06769481-0.23926912$

$1 \quad 3.27679351 \quad 0.35352287-1.29645866$

$\begin{array}{lllll}1 & 3.31514732 & 1.00092137 & 0.32897437\end{array}$

$\begin{array}{lllll}6 & 1.97532480 & -0.65458082 & 0.08699015\end{array}$

$1 \quad 1.98096552-0.949773991 .14119257$

$1 \quad 1.92374204-1.58475796-0.48790437$

$\begin{array}{lllll}6 & 0.73274827 & 0.18044031 & -0.19464064\end{array}$

$1 \quad 0.728767640 .47837988-1.24804136$

$\begin{array}{lllll}1 & 0.78272128 & 1.10906619 & 0.38289137\end{array}$

$\begin{array}{lllll}6 & -0.56844345 & -0.54299893 & 0.12822058\end{array}$

$\begin{array}{lllll}1 & -0.56433360 & -0.84128819 & 1.18150507\end{array}$

$1-0.61862192-1.47144129-0.44958837$

$\begin{array}{lllll}6 & -1.81103914 & 0.29216411 & -0.15311748\end{array}$

$\begin{array}{llll}1 & -1.81477051 & 0.59095912 & -1.20625617\end{array}$

$\begin{array}{lllll}1 & -1.76117353 & 1.22033702 & 0.42515052\end{array}$

$\begin{array}{llll}6 & -3.11231678 & -0.43158628 & 0.16884612\end{array}$

$1-3.10839254-0.730714461 .22191417$

$1-3.16220521-1.35959447-0.40973618$

$\begin{array}{lllll}6 & -4.35489559 & 0.40357922 & -0.11204175\end{array}$

$1-4.359142750 .70346026-1.16502643$

$\begin{array}{lllll}1 & -4.30585778 & 1.33157661 & 0.46686472\end{array}$

$\begin{array}{llll}6 & -5.65720854 & -0.31991833 & 0.20896607\end{array}$

$1-5.65148162-0.619107201 .26076532$

$1-5.70511404-1.24612065-0.37040902$

$\begin{array}{lllll}6 & -6.89239219 & 0.52476407 & -0.07506513\end{array}$

$1-6.936011780 .81141057-1.12739390$

$\begin{array}{lllll}1 & -6.88236518 & 1.44239840 & 0.51595893\end{array}$

$\begin{array}{lllll}1 & -7.80969365 & -0.01447890 & 0.16195233\end{array}$ energy $\quad 0.486614$

Symmetry point group: C1 moment of inertia 2.4151

Cartesian coordinate of structure 36

$6 \quad 5.69813329-1.088635940 .95028045$

$1 \quad 6.77713625-1.17075737 \quad 1.08218528$

$15.33834991-0.336747351 .65306122$

$1 \quad 5.25376329-2.045425361 .23224226$

$65.34599620-0.73047834-0.48944190$ 
$15.81333880-1.45422659-1.16084873$

$1 \quad 5.779854380 .24201668-0.73922309$

$63.84531673-0.69586934-0.77230990$

$13.41255117-1.67449617-0.53976982$

$13.69010263-0.54123908-1.84378085$

$6 \quad 3.084602160 .38186146-0.00759718$

$13.550799601 .35463450-0.19664541$

$\begin{array}{lllll}1 & 3.16963667 & 0.20701039 & 1.06834662\end{array}$

$\begin{array}{llll}6 & 1.60856693 & 0.44870078 & -0.38008392\end{array}$

$1 \quad 1.15661835-0.53223831-0.21047719$

$1 \quad 1.513590690 .64721155-1.45290192$

$\begin{array}{lllll}6 & 0.84467237 & 1.51573817 & 0.39667047\end{array}$

$\begin{array}{llll}1 & 1.35830314 & 2.47336262 & 0.27371232\end{array}$

$1 \quad 0.888298131 .282318391 .46549653$

$\begin{array}{llll}6 & -0.61410501 & 1.68024209 & -0.02296024\end{array}$

$\begin{array}{lllll}1 & -0.65712166 & 1.93171281 & -1.08770587\end{array}$

$\begin{array}{lllll}1 & -1.04076235 & 2.53549855 & 0.50850666\end{array}$

$\begin{array}{lllll}6 & -1.48767170 & 0.45780132 & 0.23813355\end{array}$

$\begin{array}{lllll}1 & -1.39672537 & 0.16879785 & 1.29052955\end{array}$

$1-1.12364535-0.39281174-0.34416823$

$\begin{array}{llll}6 & -2.95585747 & 0.69155332 & -0.09516485\end{array}$

$\begin{array}{llll}1 & -3.04426471 & 0.99214472 & -1.14415790\end{array}$

$\begin{array}{lllll}1 & -3.33090447 & 1.53438115 & 0.49427793\end{array}$

$\begin{array}{llll}6 & -3.83461803 & -0.52738843 & 0.15434694\end{array}$

$1-3.74384075-0.830593431 .20247584$

$1-3.46228780-1.36961768-0.43786998$

$6-5.30407056-0.29318877-0.17482601$

$\begin{array}{llll}1 & -5.39324971 & 0.00940215 & -1.22186685\end{array}$

$\begin{array}{lllll}1 & -5.67456422 & 0.54808643 & 0.41746070\end{array}$

$\begin{array}{llll}6 & -6.17297968 & -1.51816023 & 0.07868793\end{array}$

$\begin{array}{llll}1 & -6.12375245 & -1.82197557 & 1.12594401\end{array}$

$1-5.84098211-2.36441532-0.52554671$

$1-7.21815313-1.32509026-0.16368839$ energy $\quad 0.912190$

Symmetry point group: C1 moment of inertia 2.2352

Cartesian coordinate of structure 37

$\begin{array}{llll}6 & -4.79520628 & -1.55420634 & 0.99355082\end{array}$

$\begin{array}{lllll}1 & -5.21124617 & -2.46747057 & 1.41927087\end{array}$

$\begin{array}{llll}1 & -4.24483846 & -1.04464317 & 1.78486464\end{array}$

$\begin{array}{lllll}1 & -5.62885681 & -0.91155731 & 0.70298928\end{array}$

$6-3.90452120-1.86222010-0.20510723$

$1-4.46882030-2.46021037-0.92412676$

$1-3.06406136-2.484159130 .11624518$

$6-3.36541790-0.62108219-0.91340793$

$1-4.20541469-0.00930276-1.25847327$

$1-2.82601759-0.93229192-1.81255842$

$\begin{array}{llll}6 & -2.43751696 & 0.23727705 & -0.06056692\end{array}$ 
$\begin{array}{llll}1 & -1.64017170 & -0.39665316 & 0.33950280\end{array}$

$\begin{array}{lllll}1 & -2.97858660 & 0.63006039 & 0.80500934\end{array}$

$\begin{array}{lllll}6 & -1.83350826 & 1.40185757 & -0.83699379\end{array}$

$1-2.641224351 .97375685-1.30203673$

$\begin{array}{lllll}1 & -1.23291240 & 1.00918588 & -1.66232654\end{array}$

$\begin{array}{lllll}6 & -0.99128573 & 2.35372802 & 0.01233900\end{array}$

$\begin{array}{lllll}1 & -1.64120700 & 2.84278725 & 0.74336212\end{array}$

$\begin{array}{lllll}1 & -0.60349087 & 3.14785887 & -0.63182171\end{array}$

$\begin{array}{lllll}6 & 0.17752663 & 1.70498679 & 0.75390388\end{array}$

$\begin{array}{lllll}1 & -0.20117592 & 1.00138624 & 1.50067575\end{array}$

$\begin{array}{lllll}1 & 0.70582372 & 2.48032651 & 1.31546200\end{array}$

$\begin{array}{llll}6 & 1.17194646 & 0.98558822 & -0.14977515\end{array}$

$1 \quad 1.511233411 .67078421-0.93376144$

$1 \quad 0.674944910 .15809730-0.66438529$

$\begin{array}{lllll}6 & 2.37880771 & 0.44207665 & 0.60446089\end{array}$

$12.03521630-0.232162811 .39570423$

$1 \quad 2.89120508 \quad 1.266620101 .11033166$

$63.36922046-0.29419788-0.28835405$

$1 \quad 3.71162597 \quad 0.37883721-1.08113977$

$12.85726422-1.12026269-0.79261782$

$\begin{array}{lllll}6 & 4.57716811 & -0.83779462 & 0.46519429\end{array}$

$1 \quad 4.23352402-1.509773091 .25651801$

$1 \quad 5.08767096-0.011800250 .96810457$

$65.56011650-1.57201809-0.43732175$

$15.94103019-0.91182117-1.21867107$

$15.08052762-2.42076223-0.92833732$

$1 \quad 6.41398053-1.95080061 \quad 0.12468341$ energy $\quad 0.884883$

Symmetry point group: C1 moment of inertia 2.1796

Cartesian coordinate of structure 38

$\begin{array}{llll}6 & 3.36617069 & 2.65713962 & 0.72373225\end{array}$

$1 \quad 3.433614743 .738957150 .60767977$

$\begin{array}{lllll}1 & 2.38082502 & 2.43116141 & 1.13206201\end{array}$

14.108981602 .354417751 .46463203

$6 \quad 3.60536091 \quad 1.94719693-0.60453229$

$14.550822802 .29334130-1.02796494$

$1 \quad 2.828425402 .23695067-1.31771989$

$\begin{array}{lllll}6 & 3.64290392 & 0.42436339 & -0.49496048\end{array}$

$\begin{array}{lllll}1 & 4.43863947 & 0.13267401 & 0.19830250\end{array}$

$1 \quad 3.915478740 .00310109-1.46670196$

$62.33064858-0.20542841-0.04005476$

$1 \quad 1.527844130 .14094167-0.69740046$

$\begin{array}{lllll}1 & 2.07793161 & 0.14077162 & 0.96592099\end{array}$

$62.38239644-1.72918510-0.04442560$

$13.26679996-2.049544160 .51433033$

$12.53332884-2.07536185-1.07207371$

$6 \quad 1.15833032-2.433190130 .53990190$ 
$1 \quad 1.05649147-2.168390431 .59705656$

$1 \quad 1.35297057-3.507333780 .51768103$

$6-0.16750996-2.15097363-0.17903510$

$1-0.79430105-3.04581058-0.14138140$

$1 \quad 0.02610084-1.96771610-1.24117962$

$\begin{array}{lllll}6 & -0.97513697 & -0.99079809 & 0.39737936\end{array}$

$\begin{array}{lllll}1 & -0.38716142 & -0.07042057 & 0.37654025\end{array}$

$\begin{array}{llll}1 & -1.17922155 & -1.19317112 & 1.45363182\end{array}$

$6-2.29272359-0.76144488-0.33146403$

$1-2.88597820-1.68127564-0.30095497$

$1-2.09272466-0.56592036-1.38992785$

$\begin{array}{lllll}6 & -3.11221715 & 0.38521827 & 0.24635869\end{array}$

$\begin{array}{lllll}1 & -2.53098741 & 1.31138423 & 0.19087336\end{array}$

$\begin{array}{lllll}1 & -3.28893328 & 0.20230551 & 1.31125807\end{array}$

$\begin{array}{lllll}6 & -4.45046511 & 0.58851166 & -0.45388310\end{array}$

$1-5.02868093-0.33770592-0.39420883$

$1-4.275871560 .76997175-1.51798703$

$\begin{array}{lllll}6 & -5.26018442 & 1.73612858 & 0.13519433\end{array}$

$\begin{array}{lllll}1 & -4.72042533 & 2.68089412 & 0.04862711\end{array}$

$\begin{array}{lllll}1 & -5.46091161 & 1.56753194 & 1.19478242\end{array}$

$\begin{array}{llll}1 & -6.21850018 & 1.85301709-0.37114455\end{array}$ energy $\quad 2.41184$

Symmetry point group: C1 moment of inertia 2.1313

Cartesian coordinate of structure $\quad 39$

$\begin{array}{lllll}6 & 4.62590306 & 2.27991636 & 0.08265063\end{array}$

$15.255826613 .08439455-0.29734559$

$13.612460632 .45991299-0.27686126$

$\begin{array}{lllll}1 & 4.60937181 & 2.35522904 & 1.17188118\end{array}$

$\begin{array}{llll}6 & 5.14877787 & 0.91723496 & -0.35885924\end{array}$

$1 \quad 6.197520050 .82724395-0.06723945$

$1 \quad 5.129361240 .85607099-1.45076628$

$\begin{array}{llll}6 & 4.37475151 & -0.26469230 & 0.22191349\end{array}$

$14.40595974-0.212794791 .31531752$

$14.88480963-1.19210174-0.05337471$

$6 \quad 2.92172763-0.35062214-0.23264309$

$12.88659179-0.36390930-1.32756569$

$\begin{array}{lllll}1 & 2.38666001 & 0.54803078 & 0.08044554\end{array}$

$\begin{array}{lllll}6 & 2.20637617 & -1.58476443 & 0.30704089\end{array}$

$12.20300815-1.547639091 .40135246$

$1 \quad 2.78414458-2.47254760 \quad 0.03510835$

$6 \quad 0.77424062-1.75419065-0.19474890$

$1 \quad 0.38993072-2.717851670 .15104783$

$1 \quad 0.77878278-1.80348470-1.28852356$

$\begin{array}{lllll}6 & -0.18569843 & -0.65695811 & 0.25276388\end{array}$

$1 \quad 0.140927930 .30907187-0.14123104$

$\begin{array}{llll}1 & -0.14892277 & -0.56987379 & 1.34380513\end{array}$

$\begin{array}{lll}6 & -1.62351789-0.90637703-0.18499271\end{array}$ 


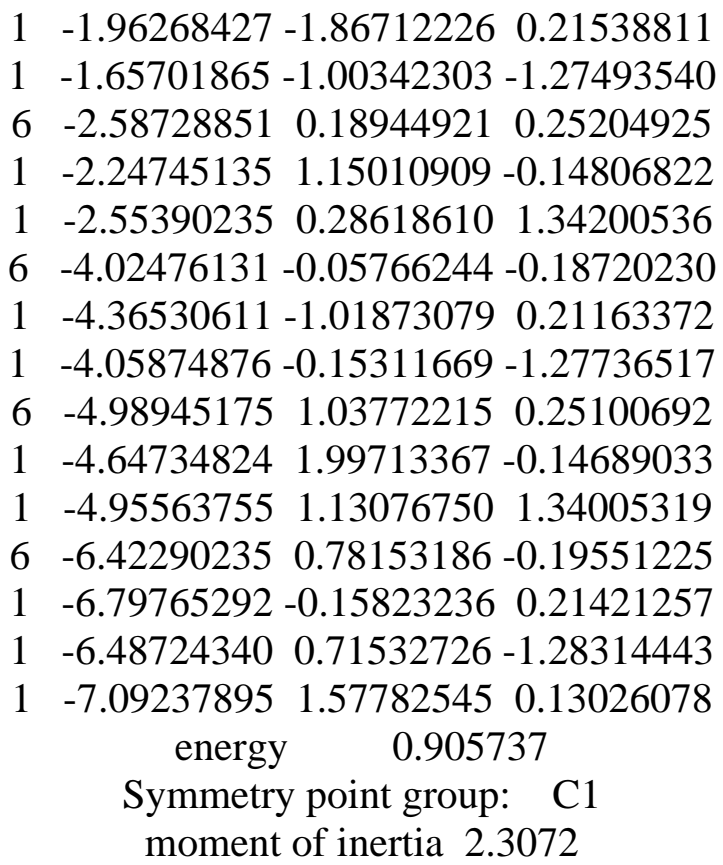

Cartesian coordinate of structure $\quad 40$ $\begin{array}{llll}6 & -2.63227060 & 2.86399743 & 0.31945943\end{array}$

$\begin{array}{lllll}1 & -2.97276784 & 3.81943947 & 0.71891580\end{array}$

$\begin{array}{lllll}1 & -1.94550665 & 2.43058597 & 1.04694257\end{array}$

$1-2.065015323 .06640082-0.59144489$

$\begin{array}{lllll}6 & -3.80887459 & 1.93785346 & 0.03053366\end{array}$

$\begin{array}{lllll}1 & -4.51681838 & 2.45468250 & -0.62113962\end{array}$

$\begin{array}{lllll}1 & -4.34571640 & 1.72961645 & 0.96044671\end{array}$

$\begin{array}{lllll}6 & -3.41086641 & 0.61662484 & -0.62459998\end{array}$

$\begin{array}{lllll}1 & -2.88244997 & 0.82538860 & -1.56093375\end{array}$

$\begin{array}{llll}1 & -4.31599625 & 0.06875719 & -0.90186975\end{array}$

$\begin{array}{lllll}6 & -2.54120315 & -0.27995334 & 0.24878130\end{array}$

$1-3.05164599-0.461555291 .20040536$

$\begin{array}{lllll}1 & -1.61227962 & 0.23447601 & 0.49822486\end{array}$

$6-2.22624862-1.62200464-0.40723954$

$1-1.80083789-1.44834502-1.40136942$

$1-3.16977940-2.14721653-0.57521966$

$\begin{array}{llll}6 & -1.27714896 & -2.51680779 & 0.40124371\end{array}$

$\begin{array}{llll}1 & -1.56428942 & -3.56150458 & 0.26718613\end{array}$

$\begin{array}{llll}1 & -1.40402625 & -2.30407736 & 1.46745214\end{array}$

$\begin{array}{llll}6 & 0.20120701-2.38930576 & 0.03238022\end{array}$

$10.33408702-2.70419570-1.00780096$

$1 \quad 0.77470480-3.096973140 .63814510$

$\begin{array}{llll}6 & 0.80251162 & -0.99909210 & 0.20417122\end{array}$

$1 \quad 0.61173460-0.642078001 .22159472$

$1 \quad 0.30303374-0.29404807-0.46621576$

$62.30034005-0.96048486-0.07135743$

$12.49090864-1.33172672-1.08353395$

$1 \quad 2.81126341-1.651767460 .60645369$

$\begin{array}{lllll}6 & 2.90478108 & 0.42980283 & 0.07697558\end{array}$ 
$\begin{array}{llll}1 & 2.71081201 & 0.80264640 & 1.08798470\end{array}$

$1 \quad 2.395360371 .12029698-0.60308260$

$64.403709940 .47218275-0.19465904$

$14.59588007 \quad 0.09914744-1.20448518$

$14.91116328-0.217146320 .48586579$

$64.99605373 \quad 1.86724402-0.04405762$

$\begin{array}{lllll}1 & 4.84258441 & 2.24975356 & 0.96668181\end{array}$

$14.525739972 .56810806-0.73618694$

$1 \quad 6.067910451 .87099371-0.24280602$ energy $\quad 2.29122$

Symmetry point group: C1 moment of inertia 2.1163

Cartesian coordinate of structure 41

$6-5.67738235-1.38125557-0.64210212$

$1-6.71357869-1.71986622-0.64507324$

$1-5.52737933-0.77576148-1.53632309$

$1-5.03791092-2.26229668-0.72742528$

$\begin{array}{llll}6 & -5.35736504 & -0.60027304 & 0.62785996\end{array}$

$\begin{array}{llll}1 & -5.61895792 & -1.20915037 & 1.49611867\end{array}$

$\begin{array}{lllll}1 & -5.99056116 & 0.29030188 & 0.67674843\end{array}$

$\begin{array}{llll}6 & -3.89317956 & -0.18068740 & 0.74414002\end{array}$

$1-3.26666334-1.076730900 .72108869$

$\begin{array}{lllll}1 & -3.73212123 & 0.28091263 & 1.72293677\end{array}$

$\begin{array}{lllll}6 & -3.43939772 & 0.79712377 & -0.33688771\end{array}$

$\begin{array}{lllll}1 & -4.15372987 & 1.62463871 & -0.38130029\end{array}$

$1-3.473652010 .31072556-1.31569524$

$\begin{array}{lllll}6 & -2.03855488 & 1.36339246 & -0.11630972\end{array}$

$\begin{array}{lllll}1 & -2.00292027 & 1.87256960 & 0.85235316\end{array}$

$\begin{array}{llll}1 & -1.84382382 & 2.13075608 & -0.87076526\end{array}$

$\begin{array}{llll}6 & -0.92366020 & 0.32468029 & -0.17683219\end{array}$

$1-0.99475489-0.22450499-1.12167432$

$\begin{array}{lllll}1 & -1.05927621 & -0.41515036 & 0.61655527\end{array}$

$\begin{array}{lllll}6 & 0.46704961 & 0.93389145 & -0.05090982\end{array}$

$\begin{array}{lllll}1 & 0.53321329 & 1.49045820 & 0.88950472\end{array}$

$1 \quad 0.61402137 \quad 1.66875114-0.84891052$

$61.58666178-0.09769329-0.10445269$

$1 \quad 1.52060043-0.65405920-1.04493621$

$\begin{array}{lllll}1 & 1.43955727 & -0.83259519 & 0.69345837\end{array}$

$\begin{array}{lllll}6 & 2.97721696 & 0.51126566 & 0.02316900\end{array}$

$\begin{array}{lllll}1 & 3.04343829 & 1.06621781 & 0.96449101\end{array}$

$13.123847521 .24745745-0.77367430$

$64.09708034-0.51984181-0.03259055$

$14.03238321-1.07399190-0.97463306$

$1 \quad 3.95018277-1.257352290 .76316808$

$\begin{array}{lllll}6 & 5.48839459 & 0.08828025 & 0.09747589\end{array}$

15.551796030 .640495951 .03920655

$15.633537760 .82510814-0.69741131$

$\begin{array}{llll}6 & 6.59977630 & -0.95155298 & 0.03925943\end{array}$ 
$1 \quad 6.57606202-1.49610238-0.90645045$

$1 \quad 6.49309356-1.682637030 .84276749$

$17.58375746-0.492172930 .13495843$ energy $\quad 0.903218$

Symmetry point group: C1 moment of inertia 2.1979

Cartesian coordinate of structure 42

$6-5.97459721-0.01793146-0.33273789$

$1-7.01295037-0.34957458-0.34708288$

$\begin{array}{lllll}1 & -5.86172067 & 0.67127458 & 0.50450739\end{array}$

$1 \quad-5.790680180 .54352467-1.25110953$

$6-5.02025350-1.20194660-0.22099303$

$1-5.23984614-1.91341864-1.02014352$

$1 \quad-5.20479153-1.730696180 .71859135$

$6-3.54411824-0.81663880-0.29718686$

$1-3.35928443-0.31349161-1.25044531$

$1-2.93862379-1.72787532-0.30929598$

$\begin{array}{lllll}6 & -3.07195539 & 0.06735662 & 0.85446897\end{array}$

$\begin{array}{llll}1 & -3.38084777 & -0.39272968 & 1.79785219\end{array}$

$\begin{array}{lllll}1 & -3.57412094 & 1.03768320 & 0.80773266\end{array}$

$\begin{array}{lllll}6 & -1.56202798 & 0.29337650 & 0.88343452\end{array}$

$\begin{array}{lllll}1 & -1.06064485 & -0.67492414 & 0.96546393\end{array}$

$\begin{array}{lllll}1 & -1.30295379 & 0.85039707 & 1.78877957\end{array}$

$\begin{array}{lllll}6 & -1.02584853 & 1.05366933 & -0.32720001\end{array}$

$\begin{array}{lllll}1 & -1.63108959 & 1.95379842 & -0.47041541\end{array}$

$\begin{array}{llll}1 & -1.15567187 & 0.45128439 & -1.23054023\end{array}$

$\begin{array}{lllll}6 & 0.44214123 & 1.45652713 & -0.20908617\end{array}$

$\begin{array}{lllll}1 & 0.57457005 & 2.08391751 & 0.67843820\end{array}$

$1 \quad 0.708305342 .08147688-1.06615900$

$\begin{array}{llll}6 & 1.41209503 & 0.28175382 & -0.14042108\end{array}$

$1 \quad 1.23724238-0.37940906-0.99581184$

$1 \quad 1.21108802-0.315931730 .75266689$

$6 \quad 2.87308736 \quad 0.71349586-0.12706910$

$\begin{array}{lllll}1 & 3.04342554 & 1.38402671 & 0.72142582\end{array}$

$13.084856341 .30190058-1.02562905$

$63.84826231-0.45407500-0.04882226$

$13.67650797-1.12645835-0.89567457$

$13.63862503-1.041142640 .85124676$

$65.31017012-0.02379219-0.03805993$

$1 \quad 5.480213980 .647683750 .80812215$

$1 \quad 5.518337860 .56198822-0.93769890$

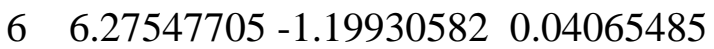

$1 \quad 6.14507670-1.86948441-0.81100447$

$1 \quad 6.10703198-1.782910390 .94758617$

$17.31335093-0.865845570 .04670551$ energy $\quad 1.30823$

Symmetry point group: C1 moment of inertia 2.0909 
Cartesian coordinate of structure 43

$\begin{array}{llll}6 & -5.79506340 & -0.83022794 & 0.90164021\end{array}$

$1-6.84811118-1.111395650 .92167360$

$1-5.20934173-1.745116670 .99461750$

$1-5.59596952-0.218308491 .78389470$

$6-5.45308747-0.06895562-0.37455246$

$1-6.134225390 .77872569-0.47718044$

$1-5.63234483-0.71204642-1.24100873$

$\begin{array}{lllll}6 & -4.01582986 & 0.44643213 & -0.42186059\end{array}$

$\begin{array}{lllll}1 & -3.85255643 & 1.11164095 & 0.43050593\end{array}$

$1-3.887431091 .05990245-1.31862720$

$6-2.96080380-0.65706473-0.43056118$

$1-3.22108082-1.38103295-1.20849895$

$1-2.98694092-1.203785090 .51615922$

$6-1.53643033-0.16155616-0.67409828$

$1-1.499372530 .38025571-1.62528831$

$1-0.88376954-1.02921506-0.79067048$

$\begin{array}{lllll}6 & -0.99197573 & 0.73814563 & 0.43215321\end{array}$

$\begin{array}{lllll}1 & -1.03624147 & 0.19976880 & 1.38501345\end{array}$

$\begin{array}{lllll}1 & -1.64218695 & 1.60837575 & 0.54299679\end{array}$

$\begin{array}{lllll}6 & 0.43547914 & 1.22774254 & 0.19555027\end{array}$

$\begin{array}{lllll}1 & 0.68584038 & 1.96781978 & 0.96075108\end{array}$

$1 \quad 0.481199641 .75510650-0.76292001$

$\begin{array}{llll}6 & 1.49517090 & 0.13117539 & 0.21538641\end{array}$

$1 \quad 1.30747690-0.58722752-0.58683807$

$1 \quad 1.41452132-0.429512191 .15258071$

$\begin{array}{lllll}6 & 2.91299118 & 0.67021249 & 0.07113309\end{array}$

$\begin{array}{lllll}1 & 3.11344106 & 1.38212907 & 0.87826129\end{array}$

$1 \quad 2.989372761 .23938228-0.86094539$

$\begin{array}{lllll}6 & 3.97849397 & -0.41827035 & 0.08425365\end{array}$

$13.77953708-1.12957656-0.72393635$

$13.90166568-0.988813871 .01555275$

$\begin{array}{lllll}6 & 5.39704724 & 0.11996671 & -0.05906491\end{array}$

$\begin{array}{lllll}1 & 5.59447581 & 0.83031744 & 0.74854585\end{array}$

$15.472295010 .68909310-0.98979259$

$6 \quad 6.45367759-0.97690462-0.04417978$

$16.29496643-1.68324716-0.86110322$

$1 \quad 6.41840625-1.540587220 .88988631$

$17.45835720-0.56682551-0.14842724$ energy $\quad 1.48658$

Symmetry point group: C1 moment of inertia 2.0900

Cartesian coordinate of structure $\quad 44$

$6 \quad 3.595199372 .51946331-0.36230189$

$14.192968013 .42941788-0.41684315$

$1 \quad 3.43536807 \quad 2.17036411-1.38270805$

$1 \quad 2.620805852 .784812240 .05351939$ 
$\begin{array}{llll}6 & 4.27995716 & 1.46116363 & 0.49596434\end{array}$

$\begin{array}{lllll}1 & 4.51334230 & 1.89119851 & 1.47248127\end{array}$

$\begin{array}{lllll}1 & 5.23828483 & 1.18942019 & 0.04422338\end{array}$

$\begin{array}{lllll}6 & 3.44471562 & 0.19887101 & 0.70084192\end{array}$

$\begin{array}{lllll}1 & 2.49496385 & 0.47884510 & 1.16662618\end{array}$

$1 \quad 3.95320295-0.455609781 .41496098$

$63.17870019-0.58521519-0.58043535$

$14.13208881-0.77571194-1.08174924$

$1 \quad 2.592489160 .02340745-1.27235408$

$62.47034725-1.92106070-0.35448370$

$13.12469486-2.575150550 .22861066$

$12.33047248-2.41098622-1.32213191$

$\begin{array}{llll}6 & 1.11693925 & -1.82750102 & 0.35067338\end{array}$

$1 \quad 1.25570583-1.47346417 \quad 1.37606314$

$1 \quad 0.69994244-2.834606170 .43694050$

$6 \quad 0.10407491-0.93164564-0.35277143$

$10.00586780-1.24527625-1.39742285$

$1 \quad 0.473640460 .09770629-0.37862972$

$\begin{array}{llll}6 & -1.26711042 & -0.94518446 & 0.31072699\end{array}$

$\begin{array}{lllll}1 & -1.16301149 & -0.64650195 & 1.35887489\end{array}$

$\begin{array}{llll}1 & -1.65305521 & -1.96945018 & 0.32445209\end{array}$

$\begin{array}{lll}6 & -2.27883409-0.03458933-0.37319067\end{array}$

$1-2.38044017-0.33095730-1.42213877$

$\begin{array}{llll}1 & -1.89327726 & 0.98997593 & -0.38369006\end{array}$

$\begin{array}{lllll}6 & -3.65072726 & -0.04877087 & 0.28865406\end{array}$

$\begin{array}{lllll}1 & -3.54945242 & 0.24656660 & 1.33805745\end{array}$

$\begin{array}{lllll}1 & -4.03747387 & -1.07298961 & 0.29836274\end{array}$

$\begin{array}{lllll}6 & -4.66279894 & 0.86347939 & -0.39398007\end{array}$

$\begin{array}{llll}1 & -4.76271197 & 0.56756870 & -1.44196062\end{array}$

$\begin{array}{lllll}1 & -4.27547941 & 1.88609374 & -0.40236212\end{array}$

$\begin{array}{lllll}6 & -6.03053515 & 0.84081002 & 0.27580508\end{array}$

$\begin{array}{lllll}1 & -5.96104419 & 1.16185881 & 1.31674812\end{array}$

$\begin{array}{llll}1 & -6.45196253 & -0.16607148 & 0.26929733\end{array}$

$1-6.735496411 .50061916-0.23024341$ energy $\quad 0.823172$

Symmetry point group: C1 moment of inertia 2.1165

Cartesian coordinate of structure $\quad 45$

$6-3.140040522 .58674607-0.29555202$

$\begin{array}{lllll}1 & -3.62927207 & 3.56083445 & -0.28394431\end{array}$

$\begin{array}{lllll}1 & -2.32523274 & 2.62082253 & 0.42808302\end{array}$

$\begin{array}{lllll}1 & -2.69900708 & 2.44669176 & -1.28478589\end{array}$

$\begin{array}{lllll}6 & -4.12938922 & 1.46959107 & 0.01890546\end{array}$

$1-4.976990091 .54205349-0.66611813$

$\begin{array}{lllll}1 & -4.53524306 & 1.61382481 & 1.02429107\end{array}$

$\begin{array}{lllll}6 & -3.53024989 & 0.06849604 & -0.08564420\end{array}$

$1-3.13477062-0.06916133-1.09640267$

$\begin{array}{lllll}1 & -4.32636397 & -0.67221284 & 0.03388313\end{array}$ 


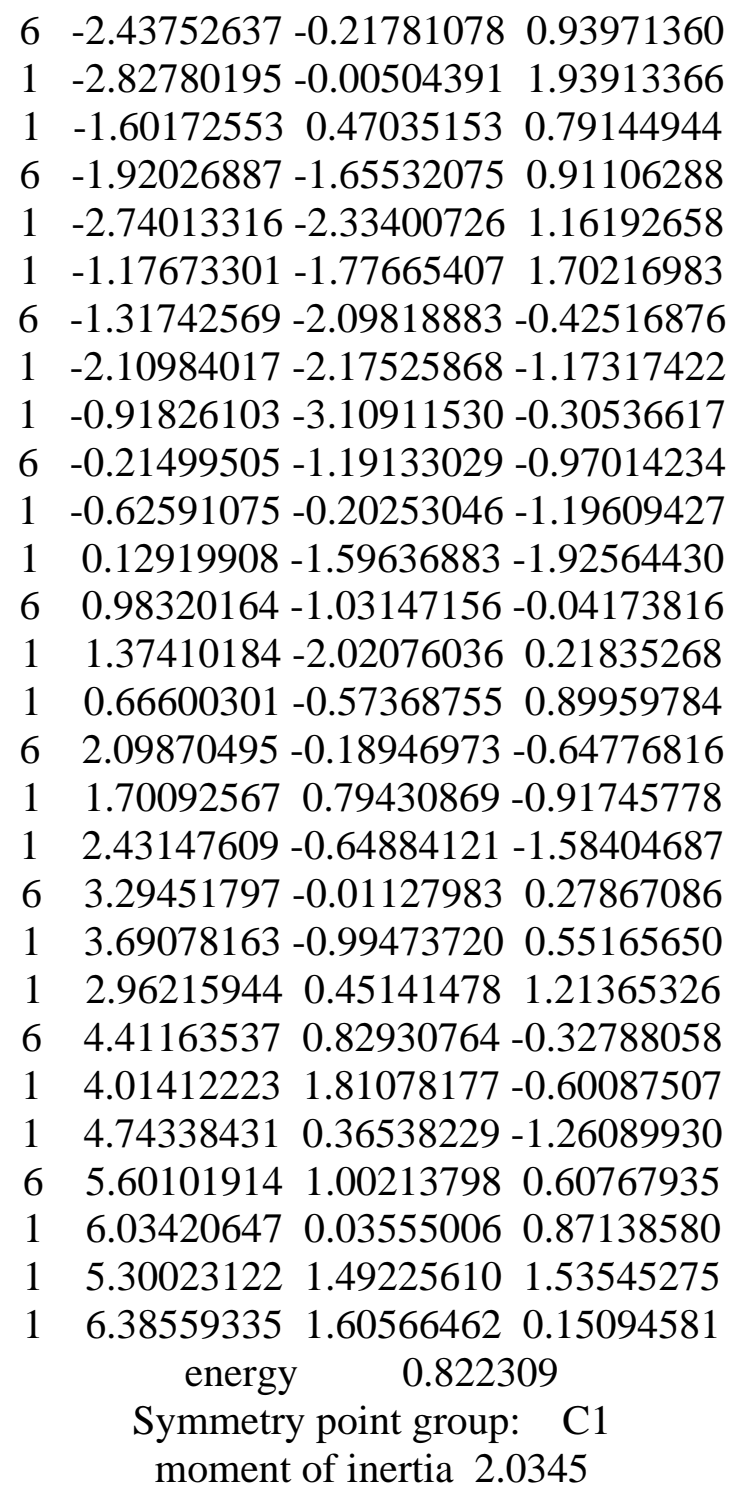

Cartesian coordinate of structure $\quad 46$

$\begin{array}{lllll}6 & -0.85831549 & 2.74040824 & 0.22295578\end{array}$

$\begin{array}{lllll}1 & -0.64329380 & 3.79467900 & 0.39797584\end{array}$

$\begin{array}{lllll}1 & -0.63134795 & 2.20274532 & 1.14439813\end{array}$

$1-0.170371952 .38299012-0.54604290$

$\begin{array}{lllll}6 & -2.30700142 & 2.53828785 & -0.21016031\end{array}$

$1-2.497093423 .14501820-1.09815015$

$\begin{array}{lllll}1 & -2.97739251 & 2.91592982 & 0.56720315\end{array}$

$\begin{array}{lllll}6 & -2.66252684 & 1.08464822 & -0.51928920\end{array}$

$\begin{array}{llll}1 & -1.95579770 & 0.70022324 & -1.25988467\end{array}$

$1-3.647938461 .04763263-0.99226414$

$\begin{array}{lllll}6 & -2.68165959 & 0.17635917 & 0.70643595\end{array}$

$\begin{array}{lllll}1 & -3.41333409 & 0.57052964 & 1.41754694\end{array}$

$\begin{array}{lllll}1 & -1.71932134 & 0.21307467 & 1.22180396\end{array}$

$\begin{array}{lllll}6 & -3.03711874-1.27788599 & 0.40081785\end{array}$

$1-4.02564563-1.30687245-0.06709820$

$\begin{array}{llll}1 & -3.13309653 & -1.81717521 & 1.34741119\end{array}$ 
$6-2.05592990-2.04780472-0.48839842$

$1-2.02430962-1.60322182-1.48692417$

$1-2.46433078-3.05100835-0.62544088$

$\begin{array}{llll}6 & -0.62778759 & -2.16014036 & 0.06124393\end{array}$

$1-0.19770070-3.11700010-0.24570149$

$1-0.66192087-2.185507901 .15560284$

$6 \quad 0.32082782-1.05247933-0.38894222$

$1-0.08263390-0.07551127-0.11892333$

$1 \quad 0.38273532-1.06063490-1.48205125$

$\begin{array}{llll}6 & 1.72068901 & -1.18083171 & 0.19613879\end{array}$

$12.15044552-2.14632759-0.08963902$

$1 \quad 1.65485145-1.189188571 .28900465$

$62.65456170-0.05950272-0.24119345$

$\begin{array}{lllll}1 & 2.21956569 & 0.90341647 & 0.04636261\end{array}$

$12.71967064-0.04800837-1.33411196$

$64.05690150-0.17170493 \quad 0.34418111$

$14.49065200-1.133025080 .05515251$

$13.98986802-0.184019051 .43559164$

$\begin{array}{lllll}6 & 4.97748837 & 0.95815012 & -0.09825603\end{array}$

$\begin{array}{lllll}1 & 4.57967957 & 1.92783954 & 0.20657828\end{array}$

$1 \quad 5.083800720 .97264486-1.18452272$

$\begin{array}{lllll}1 & 5.97348732 & 0.85575411 & 0.33292035\end{array}$ energy $\quad 1.72539$

Symmetry point group: C1 moment of inertia 2.0789

Cartesian coordinate of structure $\quad 47$ $\begin{array}{lllll}6 & -4.59798985 & 1.98024565 & 0.82586703\end{array}$

$\begin{array}{lllll}1 & -5.33467361 & 2.78248228 & 0.78083838\end{array}$

$\begin{array}{lllll}1 & -5.06524056 & 1.13360653 & 1.32937434\end{array}$

$\begin{array}{lllll}1 & -3.77025416 & 2.32186795 & 1.45092690\end{array}$

$\begin{array}{lllll}6 & -4.10602759 & 1.60652684 & -0.56820709\end{array}$

$1-3.744345772 .50601546-1.07131450$

$\begin{array}{lllll}1 & -4.94609393 & 1.23923936-1.16466490\end{array}$

$\begin{array}{lllll}6 & -2.99319247 & 0.55987497 & -0.57282431\end{array}$

$\begin{array}{lllll}1 & -2.14790625 & 0.94308272 & 0.00634875\end{array}$

$1-2.630859450 .42960596-1.59660657$

$6-3.41967560-0.79806987-0.02300331$

$1-4.34301253-1.10318199-0.52511462$

$\begin{array}{lllll}1 & -3.66787502 & -0.70108437 & 1.03754771\end{array}$

$6-2.38682877-1.91488450-0.17719839$

$1-2.19230488-2.08485801-1.24089670$

$\begin{array}{lllll}1 & -2.83620097 & -2.83664281 & 0.19733897\end{array}$

$\begin{array}{llll}6 & -1.05538649-1.67201788 & 0.54707506\end{array}$

$\begin{array}{llll}1 & -0.67226428 & -2.62070847 & 0.93128091\end{array}$

$\begin{array}{llll}1 & -1.23090322 & -1.04347418 & 1.42652752\end{array}$

$6 \quad 0.03324595-1.04094641-0.31700137$

$1-0.32783984-0.10870880-0.75726106$

$1 \quad 0.24776707-1.70795686-1.15804239$ 


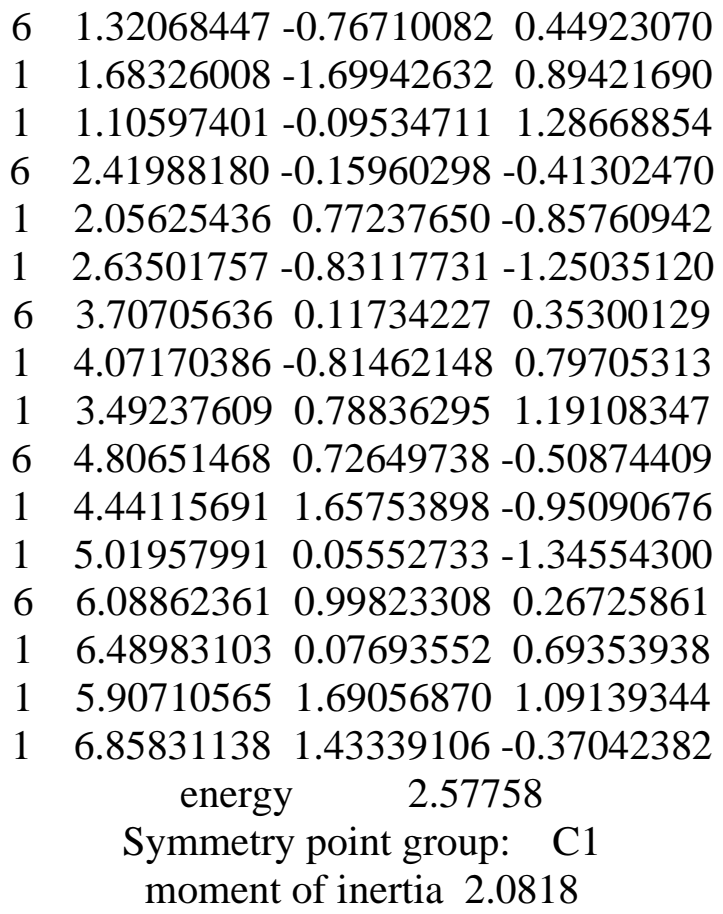

Cartesian coordinate of structure $\quad 48$

$\begin{array}{llll}6 & -2.96441908 & 2.44604654 & 1.06416043\end{array}$

$\begin{array}{lllll}1 & -3.37842556 & 3.43337715 & 1.26937844\end{array}$

$\begin{array}{lllll}1 & -3.64415253 & 1.71015054 & 1.49429089\end{array}$

$\begin{array}{lllll}1 & -2.01256550 & 2.36682433 & 1.59366772\end{array}$

$\begin{array}{lllll}6 & -2.76925521 & 2.23022512 & -0.43290710\end{array}$

$\begin{array}{lllll}1 & -2.15885798 & 3.04350153 & -0.83159411\end{array}$

$\begin{array}{lllll}1 & -3.73555271 & 2.29542708 & -0.94140327\end{array}$

$\begin{array}{lllll}6 & -2.10447657 & 0.90023889 & -0.78142656\end{array}$

$\begin{array}{lllll}1 & -1.15193857 & 0.83429695 & -0.25022358\end{array}$

$\begin{array}{lllll}1 & -1.85789708 & 0.89136874 & -1.84682435\end{array}$

$6-2.96710957-0.32464292-0.47909220$

$1-3.90244371-0.21593406-1.03463174$

$1-3.24620771-0.335599640 .57823769$

$6-2.31752923-1.66893175-0.83542011$

$1-1.64646316-1.53273445-1.68787498$

$1-3.09097495-2.35948364-1.17708913$

$\begin{array}{lllll}6 & -1.57501185 & -2.35226926 & 0.31669998\end{array}$

$1-1.18456004-3.31037076-0.03876493$

$1-2.30327697-2.59129571 \quad 1.09677880$

$\begin{array}{llll}6 & -0.43317416-1.55788750 & 0.94738556\end{array}$

$\begin{array}{llll}1 & -0.05885490 & -2.11150391 & 1.81286021\end{array}$

$\begin{array}{llll}1 & -0.81777663 & -0.61405763 & 1.34498267\end{array}$

$\begin{array}{llll}6 & 0.73235824 & -1.27557057 & 0.00688501\end{array}$

$10.37352694-0.77198419-0.89463870$

$11.16265236-2.22478612-0.32814675$

$\begin{array}{lllll}6 & 1.81829612 & -0.42056568 & 0.64671043\end{array}$

$12.18332694-0.913543621 .55343573$

$\begin{array}{llll}1 & 1.37958101 & 0.52822208 & 0.97392206\end{array}$ 


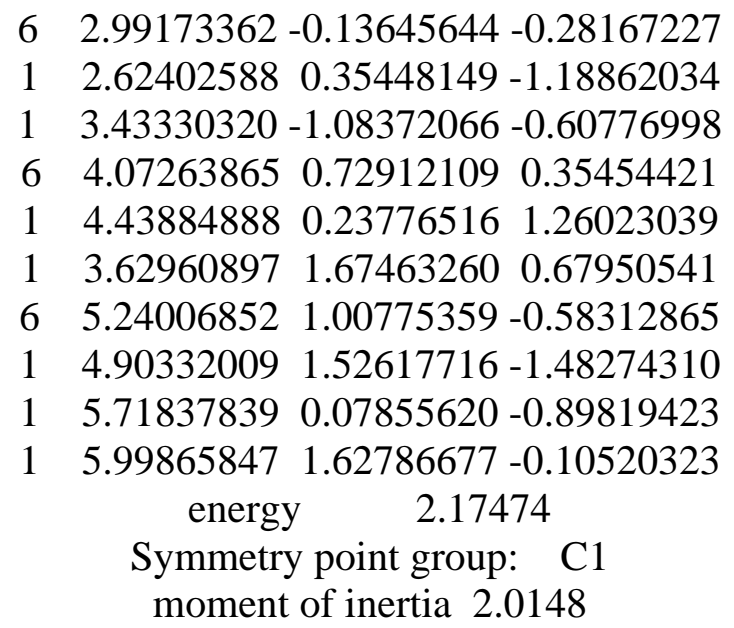

Cartesian coordinate of structure 49 $6-6.37103489-0.19897925-0.22030346$

$\begin{array}{lllll}1 & -7.32963704 & -0.64783874 & 0.04059178\end{array}$

$\begin{array}{lllll}1 & -6.28859466 & 0.73837573 & 0.33020752\end{array}$

$\begin{array}{lllll}1 & -6.39477209 & 0.04274070 & -1.28488865\end{array}$

$\begin{array}{llll}6 & -5.21809193 & -1.14591962 & 0.09483923\end{array}$

$1-5.40176089-2.10362137-0.39717495$

$1-5.20002586-1.353740031 .16858059$

$6-3.84639563-0.62885522-0.33696440$

$1-3.86354429-0.41502895-1.41119169$

$1-3.11534033-1.42761486-0.19563630$

$\begin{array}{lllll}6 & -3.38529836 & 0.61862917 & 0.41153098\end{array}$

$\begin{array}{lllll}1 & -3.35877043 & 0.40191267 & 1.48483195\end{array}$

$\begin{array}{lllll}1 & -4.12183665 & 1.41387479 & 0.27872847\end{array}$

$\begin{array}{llll}6 & -2.02103600 & 1.14531192 & -0.02981431\end{array}$

$\begin{array}{lllll}1 & -1.83536420 & 2.10228813 & 0.46584708\end{array}$

$\begin{array}{llll}1 & -2.04769230 & 1.35976522 & -1.10319030\end{array}$

$\begin{array}{lllll}6 & -0.85295169 & 0.21027956 & 0.26529591\end{array}$

$1-0.97712372-0.72904266-0.27983669$

$1-0.86095168-0.050446041 .32889586$

$\begin{array}{lllll}6 & 0.49891633 & 0.81344809 & -0.09535332\end{array}$

$\begin{array}{lllll}1 & 0.63720884 & 1.74834667 & 0.45713212\end{array}$

$1 \quad 0.501497681 .08497532-1.15591796$

$6 \quad 1.67141091-0.116815780 .18829716$

$11.53444143-1.05051716-0.36650850$

$1 \quad 1.66715561-0.390598801 .24822982$

$\begin{array}{lllll}6 & 3.02346236 & 0.48700214 & -0.16967286\end{array}$

$\begin{array}{llll}1 & 3.16053131 & 1.42065592 & 0.38523423\end{array}$

$1 \quad 3.027540750 .76090907-1.22958320$

$\begin{array}{lllll}6 & 4.19594910 & -0.44321601 & 0.11386149\end{array}$

$14.05960299-1.37713752-0.44103878$

$14.19231973-0.717541131 .17379651$

$\begin{array}{lllll}6 & 5.54896139 & 0.16010662 & -0.24371383\end{array}$

$1 \quad 5.68370782 \quad 1.09272252 \quad 0.31116139$

$1 \quad 5.55130237 \quad 0.43323753-1.30259137$ 


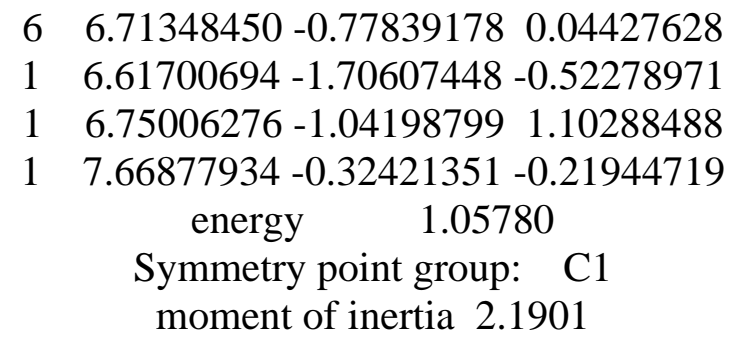

Cartesian coordinate of structure $\quad 50$

$6 \quad 6.02578737-1.14420193-0.26389278$

$17.05872805-1.266238460 .06258725$

$1 \quad 5.47262130-2.023579660 .06653605$

$1 \quad 6.01954885-1.13950985-1.35581438$

$\begin{array}{lllll}6 & 5.42507045 & 0.14457808 & 0.28702263\end{array}$

$\begin{array}{lllll}1 & 6.07296827 & 0.98130227 & 0.01661327\end{array}$

$\begin{array}{lllll}1 & 5.41827952 & 0.10547907 & 1.38011707\end{array}$

$\begin{array}{lllll}6 & 4.01075582 & 0.43985078 & -0.21099929\end{array}$

$14.014821380 .47733893-1.30571838$

$\begin{array}{lllll}1 & 3.72694888 & 1.43824573 & 0.12798909\end{array}$

$\begin{array}{lllll}6 & 2.96380869 & -0.57159502 & 0.24749607\end{array}$

$12.96247676-0.614908391 .34198318$

$13.25053791-1.56759841-0.09633855$

$61.54715958-0.27978085-0.24630566$

$10.91648146-1.14022413-0.01362793$

$1 \quad 1.55630164-0.19393290-1.33824849$

$\begin{array}{lllll}6 & 0.92078386 & 0.97876655 & 0.34827941\end{array}$

$\begin{array}{lllll}1 & 1.55641915 & 1.83966490 & 0.13156669\end{array}$

$\begin{array}{lllll}1 & 0.89610610 & 0.88342559 & 1.43906666\end{array}$

$\begin{array}{lllll}6 & -0.48709773 & 1.27842193 & -0.16298040\end{array}$

$\begin{array}{llll}1 & -0.46151388 & 1.37489532 & -1.25333582\end{array}$

$\begin{array}{lllll}1 & -0.80101599 & 2.25341884 & 0.22018251\end{array}$

$\begin{array}{lllll}6 & -1.53792090 & 0.24297415 & 0.22362173\end{array}$

$\begin{array}{lllll}1 & -1.52046702 & 0.10096569 & 1.30935988\end{array}$

$1-1.28832125-0.72676377-0.21483774$

$\begin{array}{lllll}6 & -2.94542252 & 0.63237351 & -0.21094389\end{array}$

$\begin{array}{lllll}1 & -2.95866971 & 0.78689887 & -1.29469663\end{array}$

$\begin{array}{lllll}1 & -3.20812782 & 1.59664651 & 0.23586519\end{array}$

$\begin{array}{lllll}6 & -4.00171787 & -0.39954652 & 0.16270682\end{array}$

$1-3.98592896-0.557798951 .24597239$

$1-3.74221401-1.36303396-0.28790817$

$6-5.41050554-0.00783712-0.26674414$

$\begin{array}{lllll}1 & -5.42473119 & 0.15021361 & -1.34870577\end{array}$

$\begin{array}{lllll}1 & -5.66845863 & 0.95426694 & 0.18452192\end{array}$

$\begin{array}{llll}6 & -6.45813237 & -1.04665188 & 0.11160610\end{array}$

$1-6.48345100-1.200270031 .19199021$

$1-6.23872998-2.00979893-0.35281424$

$1-7.45602274-0.74321493-0.20550492$ energy $\quad 1.64151$

Symmetry point group: C1 
moment of inertia 2.1135

Cartesian coordinate of structure 51

$6 \quad 5.73050798-1.414603820 .35175974$

$1 \quad 6.80385149-1.530252010 .20009356$

$15.58196373-1.04335577 \quad 1.36597203$

$1 \quad 5.27656689-2.405886950 .29172403$

$65.12696314-0.47838272-0.68962817$

$15.38993706-0.84043251-1.68600266$

$15.580833520 .51242095-0.59602815$

$63.60735530-0.34183920-0.60334426$

$13.15080247-1.33415357-0.68666376$

$\begin{array}{lllll}1 & 3.26087411 & 0.22777682 & -1.46819849\end{array}$

$\begin{array}{lllll}6 & 3.11219107 & 0.32858120 & 0.67511057\end{array}$

$\begin{array}{lllll}1 & 3.55247648 & 1.32889758 & 0.74713731\end{array}$

$13.47501583-0.228564941 .54145922$

$\begin{array}{lllll}6 & 1.59175876 & 0.43804518 & 0.77308326\end{array}$

$\begin{array}{lllll}1 & 1.32797848 & 0.82787645 & 1.76076763\end{array}$

$1 \quad 1.16157334-0.565662640 .71636216$

$\begin{array}{llll}6 & 0.95799284 & 1.33448235 & -0.28814305\end{array}$

$11.085919850 .88774766-1.27786086$

$1 \quad 1.497790002 .28586998-0.30967550$

$\begin{array}{lllll}6 & -0.52630851 & 1.61181074-0.06121239\end{array}$

$\begin{array}{llll}1 & -0.86902207 & 2.33756781 & -0.80404896\end{array}$

$\begin{array}{lllll}1 & -0.65969101 & 2.08892877 & 0.91521916\end{array}$

$\begin{array}{lllll}6 & -1.41536288 & 0.37521348 & -0.13888655\end{array}$

$\begin{array}{lllll}1 & -1.13894259 & -0.33261364 & 0.64710602\end{array}$

$1-1.23517976-0.13835342-1.08924511$

$\begin{array}{lllll}6 & -2.89907408 & 0.69768426 & -0.01326754\end{array}$

$\begin{array}{llll}1 & -3.18663700 & 1.39411370 & -0.80747449\end{array}$

$\begin{array}{lllll}1 & -3.07512720 & 1.22441138 & 0.93020694\end{array}$

$6-3.79324248-0.53391477-0.07565194$

$1-3.50829071-1.228800130 .72099804$

$1-3.61507903-1.06331185-1.01732658$

$\begin{array}{lllll}6 & -5.27791545 & -0.21238444 & 0.04626916\end{array}$

$1-5.561543270 .48041645-0.75085267$

$\begin{array}{lllll}1 & -5.45408063 & 0.31735362 & 0.98657488\end{array}$

$6-6.16175377-1.45127724-0.01529740$

$1-5.91763373-2.145663160 .79074974$

$1-6.02542367-1.98179639-0.95945633$

$\begin{array}{lllll}1 & -7.21760427 & -1.19502447 & 0.07371440\end{array}$

energy $\quad 1.46007$

Symmetry point group: C1

moment of inertia 2.0920

Cartesian coordinate of structure $\quad 52$

$65.20277172-1.68033022-0.39076842$

$15.59278355-2.63300948-0.74944686$

$15.33588072-0.95015077-1.18939854$ 
$1 \quad 5.81889865-1.364119360 .45355525$

$\begin{array}{lllll}6 & 3.74018281 & -1.80557178 & 0.02206405\end{array}$

$13.64145322-2.620933290 .74214366$

$13.14038257-2.09171692-0.84697606$

$\begin{array}{lllll}6 & 3.15373880 & -0.53580877 & 0.63504941\end{array}$

$13.72938597-0.26582821 \quad 1.52651842$

$1 \quad 2.14139874-0.74809507 \quad 0.98331074$

$\begin{array}{lllll}6 & 3.12757857 & 0.66307807 & -0.31225787\end{array}$

$1 \quad 2.67911331 \quad 0.36135187-1.26523164$

$14.154870220 .94984399-0.54313879$

$\begin{array}{lllll}6 & 2.37519320 & 1.88180592 & 0.24137616\end{array}$

$12.884850792 .79518460-0.07105670$

$\begin{array}{lllll}1 & 2.42488207 & 1.86847456 & 1.33475744\end{array}$

$6 \quad 0.914562901 .98997316-0.19712612$

$10.880446622 .10020524-1.28595046$

$\begin{array}{lllll}1 & 0.49305253 & 2.91209238 & 0.21354766\end{array}$

$\begin{array}{lllll}6 & 0.02158840 & 0.82217595 & 0.20561812\end{array}$

$\begin{array}{lllll}1 & 0.09303459 & 0.66439295 & 1.28678977\end{array}$

$1 \quad 0.38148899-0.09846536-0.26247146$

$\begin{array}{lllll}6 & -1.43785662 & 1.03063642 & -0.17861437\end{array}$

$\begin{array}{llll}1 & -1.50599504 & 1.20043485 & -1.25801138\end{array}$

$\begin{array}{lllll}1 & -1.81034320 & 1.94401019 & 0.29604780\end{array}$

$\begin{array}{lllll}6 & -2.33613827 & -0.13852967 & 0.20446074\end{array}$

$\begin{array}{lllll}1 & -2.26630342 & -0.30918561 & 1.28356961\end{array}$

$1-1.96362250-1.05130672-0.27151187$

$\begin{array}{lllll}6 & -3.79585502 & 0.06842233 & -0.17856689\end{array}$

$\begin{array}{llll}1 & -3.86619292 & 0.23885343 & -1.25779117\end{array}$

$\begin{array}{lllll}1 & -4.16911874 & 0.98118728 & 0.29705795\end{array}$

$\begin{array}{lllll}6 & -4.69492907 & -1.10092534 & 0.20453745\end{array}$

$\begin{array}{llll}1 & -4.62426012 & -1.26943832 & 1.28270101\end{array}$

$1-4.32064072-2.01186708-0.27077716$

$6-6.15147630-0.88350941-0.18404796$

$1-6.25137118-0.74296189-1.26188371$

$\begin{array}{lllll}1 & -6.55718569 & 0.00521551 & 0.30280869\end{array}$

$\begin{array}{llll}1 & -6.77305545 & -1.73266868 & 0.10049198\end{array}$ energy $\quad 2.63602$

Symmetry point group: C1 moment of inertia 2.0704

Cartesian coordinate of structure 53

$\begin{array}{llll}6 & 4.18035984 & 2.38856743 & -0.04561239\end{array}$

$\begin{array}{lllll}1 & 4.58383727 & 3.25714149 & 0.47511023\end{array}$

14.904673561 .580253090 .05681116

$14.110016602 .63847840-1.10628903$

$\begin{array}{lllll}6 & 2.81513999 & 2.00009079 & 0.51165744\end{array}$

12.160563692 .874249930 .49004853

$\begin{array}{lllll}1 & 2.91746090 & 1.72203638 & 1.56469685\end{array}$

$\begin{array}{llll}6 & 2.13222034 & 0.85938238 & -0.24055654\end{array}$

$12.007207401 .14353002-1.29075960$ 
$\begin{array}{llll}1 & 1.12502776 & 0.73397722 & 0.15980504\end{array}$

$6 \quad 2.88417922-0.46956805-0.17755705$

$13.08853703-0.718856250 .86949425$

$13.85939579-0.33839641-0.64963862$

$62.16212105-1.64576719-0.84978296$

$12.90361259-2.31221252-1.29459490$

$1 \quad 1.56325256-1.27233697-1.68498132$

$\begin{array}{llll}6 & 1.29365532 & -2.49353838 & 0.08422762\end{array}$

$1 \quad 1.94432492-2.940768680 .84096155$

$1 \quad 0.87655453-3.32687307-0.48886854$

$\begin{array}{lllll}6 & 0.15289828 & -1.76646995 & 0.79320725\end{array}$

$1 \quad 0.55621786-0.95286557 \quad 1.40318328$

$1-0.31638086-2.458287241 .49811058$

$6-0.92037564-1.21922946-0.14033026$

$1-1.36430707-2.04748275-0.70193907$

$1-0.46892458-0.55782176-0.88461563$

$\begin{array}{lllll}6 & -2.01564295 & -0.45677716 & 0.59369782\end{array}$

$\begin{array}{lllll}1 & -1.56424803 & 0.37301965 & 1.14791697\end{array}$

$\begin{array}{lllll}1 & -2.47273066 & -1.10911741 & 1.34466408\end{array}$

$\begin{array}{lllll}6 & -3.09818458 & 0.08664745 & -0.32963243\end{array}$

$1-3.55143674-0.74196382-0.88336329$

$\begin{array}{lllll}1 & -2.63910622 & 0.73772689 & -1.08070644\end{array}$

$\begin{array}{lllll}6 & -4.18966726 & 0.85747572 & 0.40304593\end{array}$

$\begin{array}{lllll}1 & -3.73525361 & 1.68477950 & 0.95522954\end{array}$

$\begin{array}{lllll}1 & -4.64710991 & 0.20615614 & 1.15293754\end{array}$

$\begin{array}{lllll}6 & -5.26583300 & 1.39641000 & -0.53033907\end{array}$

$1-5.755414860 .58487367-1.07181415$

$\begin{array}{lllll}1 & -4.83702339 & 2.07440713-1.27054805\end{array}$

$\begin{array}{lllll}1 & -6.03397009 & 1.94301147 & 0.01699681\end{array}$ energy 2.43533

Symmetry point group: C1 moment of inertia 2.0325

Cartesian coordinate of structure 54

$65.44385491-1.47170388-0.21520156$

$15.94718155-2.42596491-0.37168809$

$15.46776070-0.93052304-1.16128303$

$1 \quad 6.02925560-0.900637210 .50831807$

$\begin{array}{llll}6 & 4.01816277-1.67876423 & 0.28438215\end{array}$

$14.04332269-2.309059291 .17606090$

$13.44604863-2.23364671-0.46488323$

$\begin{array}{lllll}6 & 3.27790006 & -0.38467200 & 0.61924369\end{array}$

$\begin{array}{lllll}1 & 3.85208788 & 0.17877532 & 1.36276604\end{array}$

$1 \quad 2.33002557-0.642100561 .09795136$

$6 \quad 3.005849090 .50810791-0.58721291$

$12.41606140-0.05245182-1.31853177$

$1 \quad 3.947445630 .75046468-1.08416934$

$6 \quad 2.299632721 .82089296-0.24553358$

$12.155428402 .38901673-1.16881508$ 
$\begin{array}{llll}1 & 2.96117346 & 2.42322375 & 0.38342156\end{array}$

$\begin{array}{lllll}6 & 0.95054982 & 1.67822981 & 0.45925135\end{array}$

$\begin{array}{llll}1 & 0.54255700 & 2.67800381 & 0.63141916\end{array}$

$\begin{array}{lllll}1 & 1.09207079 & 1.23985559 & 1.45107654\end{array}$

$\begin{array}{llll}6 & -0.07425936 & 0.85248013 & -0.30943165\end{array}$

$10.28632043-0.17395558-0.42379580$

$\begin{array}{llll}1 & -0.17605227 & 1.25326222 & -1.32349289\end{array}$

$\begin{array}{lllll}6 & -1.44122189 & 0.82187541 & 0.36224939\end{array}$

$\begin{array}{lllll}1 & -1.81631181 & 1.84490383 & 0.46710425\end{array}$

$\begin{array}{llll}1 & -1.33386052 & 0.43269253 & 1.37992750\end{array}$

$6-2.46686937-0.01579079-0.39067586$

$1-2.09178232-1.03908178-0.49399960$

$1-2.573089860 .37243975-1.40874422$

$\begin{array}{lllll}6 & -3.83370511 & -0.04682820 & 0.28100382\end{array}$

$\begin{array}{lllll}1 & -4.20938536 & 0.97627862 & 0.38500589\end{array}$

$\begin{array}{lllll}1 & -3.72792473 & -0.43550007 & 1.29907893\end{array}$

$6-4.86050864-0.88435850-0.47179500$

$1-4.48377031-1.90572019-0.57537752$

$1-4.96574558-0.49449510-1.48804092$

$\begin{array}{lllll}6 & -6.22253880 & -0.90900279 & 0.20944893\end{array}$

$\begin{array}{lllll}1 & -6.63349107 & 0.09838032 & 0.29757627\end{array}$

$1-6.14803474-1.32339367 \quad 1.21652265$

$1-6.93836852-1.51356215-0.34778030$ energy $\quad 1.07243$

Symmetry point group: C1 moment of inertia 2.2098

Cartesian coordinate of structure $\quad 55$

$\begin{array}{lllll}6 & 3.24802216 & 2.88412854 & 0.45172286\end{array}$

$\begin{array}{lllll}1 & 3.01938306 & 3.94760349 & 0.52323243\end{array}$

$1 \quad 3.247412052 .479689461 .46420276$

$\begin{array}{lllll}1 & 4.26120498 & 2.78639378 & 0.05632785\end{array}$

$\begin{array}{llll}6 & 2.24498301 & 2.16535078 & -0.44367807\end{array}$

$12.201784162 .67172900-1.41043347$

$1 \quad 1.243771502 .25096743-0.01006979$

$\begin{array}{llll}6 & 2.55887247 & 0.68867038 & -0.67495496\end{array}$

$1 \quad 3.541584830 .59428002-1.14863949$

$\begin{array}{lllll}1 & 1.83705888 & 0.29164968 & -1.39171554\end{array}$

$\begin{array}{lllll}6 & 2.53560438 & -0.15633389 & 0.59515382\end{array}$

$\begin{array}{lllll}1 & 1.60823747 & 0.03576623 & 1.14341207\end{array}$

$\begin{array}{lllll}1 & 3.34228407 & 0.16465341 & 1.25766426\end{array}$

$\begin{array}{llll}6 & 2.68932898 & -1.65690518 & 0.35182495\end{array}$

$1 \quad 2.76264473-2.157880151 .32120215$

$13.64273519-1.83485511-0.15441757$

$6 \quad 1.57713991-2.33679785-0.45222954$

$1 \quad 1.84006505-3.39313417-0.53591945$

$1 \quad 1.56421066-1.95521034-1.47677685$

$\begin{array}{llll}6 & 0.17089855-2.21744508 & 0.14958037\end{array}$

$1 \quad 0.24568703-2.173522551 .24127213$ 
$1-0.39400891-3.12612975-0.07382510$

$6-0.64422555-1.02694440-0.34969345$

$1-0.73992805-1.09455792-1.43820465$

$1-0.11435865-0.09262515-0.15255903$

$\begin{array}{lllll}6 & -2.03154528 & -0.94787949 & 0.27356053\end{array}$

$\begin{array}{llll}1 & -1.93464900 & -0.88507016 & 1.36229698\end{array}$

$\begin{array}{lllll}1 & -2.57492369 & -1.87635651 & 0.07048984\end{array}$

$\begin{array}{lllll}6 & -2.84926771 & 0.23580186 & -0.22709608\end{array}$

$\begin{array}{llll}1 & -2.94607821 & 0.17424072 & -1.31591516\end{array}$

$\begin{array}{lllll}1 & -2.30400095 & 1.16334132 & -0.02382956\end{array}$

$\begin{array}{lllll}6 & -4.23710249 & 0.32268797 & 0.39627810\end{array}$

$\begin{array}{lllll}1 & -4.13868834 & 0.38413490 & 1.48364231\end{array}$

$\begin{array}{lllll}1 & -4.78075701 & -0.60384705 & 0.19194596\end{array}$

$\begin{array}{lllll}6 & -5.04346923 & 1.51081061 & -0.11161056\end{array}$

$1-5.180952361 .45585110-1.19306508$

$\begin{array}{lllll}1 & -4.53420168 & 2.45094849 & 0.10809274\end{array}$

$\begin{array}{lllll}1 & -6.03095198 & 1.55107425 & 0.34844149\end{array}$ energy $\quad 2.20856$

Symmetry point group: C1 moment of inertia 2.0531

Cartesian coordinate of structure 56

$\begin{array}{llll}6 & -4.72384430 & -2.02864302 & 0.35242954\end{array}$

$\begin{array}{llll}1 & -5.15052352 & -2.97674071 & 0.02457232\end{array}$

$\begin{array}{lllll}1 & -4.10589351 & -2.23332431 & 1.22691858\end{array}$

$1-5.54649093-1.385119620 .67092610$

$6-3.92340101-1.37115887-0.76651065$

$1-4.55725602-1.28346160-1.65166841$

$1-3.09162150-2.02231591-1.05082573$

$\begin{array}{llll}6 & -3.37613528 & 0.01166895 & -0.41609065\end{array}$

$\begin{array}{lllll}1 & -4.20570684 & 0.66798171 & -0.13164550\end{array}$

$\begin{array}{llll}1 & -2.93706805 & 0.44836292 & -1.31610396\end{array}$

$\begin{array}{lllll}6 & -2.33688717 & 0.00586534 & 0.70048674\end{array}$

$\begin{array}{llll}1 & -1.51212229 & -0.65461414 & 0.41442604\end{array}$

$1-2.77081399-0.430381941 .60250577$

$\begin{array}{lllll}6 & -1.79414454 & 1.38994351 & 1.05584420\end{array}$

$\begin{array}{lllll}1 & -1.09356660 & 1.28724206 & 1.88776495\end{array}$

$\begin{array}{lllll}1 & -2.61582852 & 2.00661820 & 1.43042839\end{array}$

$\begin{array}{lllll}6 & -1.11181985 & 2.13975837 & -0.09155699\end{array}$

$\begin{array}{lllll}1 & -0.70754308 & 3.07636812 & 0.30223196\end{array}$

$\begin{array}{lllll}1 & -1.85897477 & 2.42581838 & -0.83531758\end{array}$

$\begin{array}{lllll}6 & 0.00885645 & 1.37227952 & -0.79123551\end{array}$

$1 \quad 0.411445381 .99705680-1.59318588$

$1-0.400096260 .48393205-1.28223033$

$\begin{array}{lllll}6 & 1.15045454 & 0.95205534 & 0.12722988\end{array}$

$\begin{array}{lllll}1 & 0.77546223 & 0.27736973 & 0.90209377\end{array}$

$\begin{array}{lllll}1 & 1.53559084 & 1.83276027 & 0.65180889\end{array}$

$\begin{array}{lllll}6 & 2.29064266 & 0.26538821 & -0.61375868\end{array}$

$12.681988990 .94001475-1.38193863$ 
$1 \quad 1.89932322-0.60696163-1.14728720$

$\begin{array}{lllll}6 & 3.42854567 & -0.17283601 & 0.29907815\end{array}$

$13.03695336-0.848509851 .06646088$

$\begin{array}{lllll}1 & 3.81974495 & 0.69866004 & 0.83403490\end{array}$

$64.56908665-0.86137306-0.44083656$

$14.96013607-0.18527353-1.20619675$

$14.17654710-1.73104173-0.97509571$

$\begin{array}{llll}6 & 5.69970463-1.29656960 & 0.48227227\end{array}$

$1 \quad 5.33901609-1.99626581 \quad 1.23845165$

$1 \quad 6.12919413-0.439783721 .00468941$

$16.50175296-1.78666256-0.06992838$ energy $\quad 1.10145$

Symmetry point group: C1 moment of inertia 2.0478

Cartesian coordinate of structure $\quad 57$

$\begin{array}{llll}6 & -5.18258363 & 1.52841041 & -0.68450246\end{array}$

$\begin{array}{lllll}1 & -5.54576987 & 2.53234437 & -0.46455747\end{array}$

$\begin{array}{lllll}1 & -4.20696388 & 1.62974399-1.16200075\end{array}$

$1 \quad-5.861068991 .07920580-1.41265515$

$\begin{array}{lllll}6 & -5.10233117 & 0.68284918 & 0.58108153\end{array}$

$\begin{array}{lllll}1 & -6.07025010 & 0.69901715 & 1.08667121\end{array}$

$\begin{array}{lllll}1 & -4.39326890 & 1.13738872 & 1.27698237\end{array}$

$\begin{array}{lllll}6 & -4.71498701 & -0.77476158 & 0.32744913\end{array}$

$1-5.50458259-1.25120334-0.26061549$

$1-4.68601177-1.301623531 .28539634$

$6-3.38040092-0.97977513-0.38889888$

$1-3.43481435-0.56897751-1.40110682$

$1-3.21378996-2.05342298-0.51177098$

$\begin{array}{lllll}6 & -2.18207990 & -0.37332364 & 0.33194098\end{array}$

$\begin{array}{llll}1 & -2.15981865 & -0.73458966 & 1.36552406\end{array}$

$\begin{array}{lllll}1 & -2.29583946 & 0.71291415 & 0.39273564\end{array}$

$\begin{array}{lllll}6 & -0.85452177-0.69235551-0.34379078\end{array}$

$1-0.88551739-0.34480931-1.38153249$

$1-0.72443130-1.77820428-0.39174105$

$\begin{array}{lllll}6 & 0.34632734 & -0.07166092 & 0.35846628\end{array}$

$\begin{array}{lllll}1 & 0.37450120 & -0.41501441 & 1.39757378\end{array}$

$\begin{array}{lllll}1 & 0.21783561 & 1.01464319 & 0.40157975\end{array}$

$61.67454443-0.39450668-0.31403241$

$1 \quad 1.64536297-0.05364532-1.35391826$

$1 \quad 1.80446911-1.48070616-0.35461640$

$\begin{array}{lllll}6 & 2.87476192 & 0.22988363 & 0.38626170\end{array}$

$12.90300047-0.109683331 .42662175$

$\begin{array}{lllll}1 & 2.74523971 & 1.31622824 & 0.42538037\end{array}$

$64.20333930-0.09426201-0.28470343$

$14.175205430 .24375528-1.32570030$

$14.33427368-1.18060122-0.32255708$

$\begin{array}{lllll}6 & 5.40422860 & 0.53204990 & 0.41394873\end{array}$

$1 \quad 5.43078794 \quad 0.194137941 .45363406$ 
$\begin{array}{llll}1 & 5.27243012 & 1.61701323 & 0.45012437\end{array}$

$\begin{array}{lllll}6 & 6.72680500 & 0.20054231 & -0.26480774\end{array}$

$1 \quad 6.735591610 .55490584-1.29719790$

$1 \quad 6.89582776-0.87763302-0.28539455$

$\begin{array}{lllll}1 & 7.56898871 & 0.66027640 & 0.25266512\end{array}$ energy $\quad 0.597890$

Symmetry point group: C1 moment of inertia 2.2355

Cartesian coordinate of structure 58

$\begin{array}{lllll}6 & -4.94598431 & -0.83699097 & 1.29661348\end{array}$

$\begin{array}{llll}1 & -5.15871288 & -1.52451981 & 2.11528234\end{array}$

$\begin{array}{llll}1 & -4.34751298 & -0.01973758 & 1.70162557\end{array}$

$1-5.89480874-0.415066340 .95891535$

$\begin{array}{llll}6 & -4.22703696 & -1.54634850 & 0.15509746\end{array}$

$1-4.80610046-2.42202975-0.14592075$

$1-3.26827724-1.929482630 .51247364$

$6-4.00521723-0.66766760-1.07683621$

$1-4.97849930-0.40140840-1.49886996$

$1-3.49106106-1.25792474-1.84058903$

$\begin{array}{lllll}6 & -3.21219967 & 0.61495852 & -0.82782644\end{array}$

$\begin{array}{lllll}1 & -3.77508807 & 1.27774018 & -0.16441149\end{array}$

$1-3.116000661 .15313917-1.77483419$

$\begin{array}{llll}6 & -1.82153027 & 0.38845875 & -0.24590117\end{array}$

$1-1.28763790-0.33182858-0.87219675$

$\begin{array}{lllll}1 & -1.90246010 & -0.06913268 & 0.74491039\end{array}$

$\begin{array}{lllll}6 & -1.01556041 & 1.67767495 & -0.12701998\end{array}$

$\begin{array}{lllll}1 & -1.60944092 & 2.41154874 & 0.42518917\end{array}$

$\begin{array}{lllll}1 & -0.86286415 & 2.09975065-1.12561333\end{array}$

$\begin{array}{lllll}6 & 0.33610356 & 1.51545051 & 0.56397701\end{array}$

$\begin{array}{lllll}1 & 0.18014671 & 1.11733979 & 1.57195507\end{array}$

$1 \quad 0.78847305 \quad 2.502578440 .69386486$

$6 \quad 1.320915590 .62008676-0.18025134$

$1 \quad 1.432894180 .98393816-1.20710721$

$10.91825933-0.39332489-0.25822300$

$\begin{array}{lllll}6 & 2.69032729 & 0.55690570 & 0.48451903\end{array}$

$\begin{array}{lllll}1 & 2.57525334 & 0.20430436 & 1.51457965\end{array}$

$\begin{array}{lllll}1 & 3.10568617 & 1.56734886 & 0.55468555\end{array}$

$6 \quad 3.67728529-0.34321827-0.24757457$

$1 \quad 3.79161987 \quad 0.00794132-1.27828978$

$13.26292723-1.35430054-0.31638858$

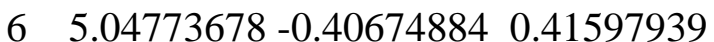

$14.93192402-0.757835031 .44513394$

$\begin{array}{lllll}1 & 5.46058797 & 0.60356358 & 0.48399036\end{array}$

$6 \quad 6.02575970-1.30942999-0.32460375$

$16.18135538-0.96086374-1.34723160$

$15.64856242-2.33227618-0.37925122$

$1 \quad 6.99717867-1.33824855 \quad 0.16928361$ energy $\quad 0.989493$ 
Symmetry point group: C1

moment of inertia 2.2268

Cartesian coordinate of structure $\quad 59$

$\begin{array}{llll}6 & 3.80724406 & 2.22338562 & 0.77432200\end{array}$

$\begin{array}{lllll}1 & 3.59953583 & 3.29189919 & 0.71707333\end{array}$

$\begin{array}{lllll}1 & 3.01539289 & 1.76540882 & 1.36856758\end{array}$

$\begin{array}{llll}1 & 4.74583257 & 2.09338516 & 1.31692100\end{array}$

$63.894305691 .60649222-0.61685716$

$14.614167192 .16882421-1.21542092$

$12.932540351 .71792544-1.12319361$

$\begin{array}{llll}6 & 4.31022619 & 0.13498845 & -0.61650758\end{array}$

$15.327614390 .05664647-0.22255828$

$14.35405147-0.21802739-1.65069077$

$\begin{array}{llll}6 & 3.40502408 & -0.80044242 & 0.18511231\end{array}$

$\begin{array}{llll}1 & 3.45495874 & -0.54794967 & 1.24814737\end{array}$

$13.80065422-1.816499630 .10203422$

$61.94800123-0.79794404-0.26240450$

$1 \quad 1.89895176-0.98283794-1.34111106$

$1 \quad 1.519355910 .19406038-0.10425807$

$\begin{array}{llll}6 & 1.10318790 & -1.84079922 & 0.46185487\end{array}$

$1 \quad 1.12661148-1.639875651 .53804581$

$1 \quad 1.56720888-2.821652630 .32647344$

$6-0.34813256-1.90939659-0.00841849$

$\begin{array}{lllll}1 & -0.82988234 & -2.77721332 & 0.45045097\end{array}$

$1-0.36784564-2.08676998-1.08871259$

$\begin{array}{lllll}6 & -1.17530709 & -0.66823218 & 0.30995780\end{array}$

$\begin{array}{lllll}1 & -0.72600074 & 0.21180932 & -0.15763448\end{array}$

$\begin{array}{lllll}1 & -1.14968210 & -0.48694850 & 1.38955342\end{array}$

$6-2.62278540-0.78305712-0.15149076$

$\begin{array}{lllll}1 & -3.08228681 & -1.66307757 & 0.30983037\end{array}$

$1-2.64244596-0.96201080-1.23137354$

$\begin{array}{lllll}6 & -3.46101599 & 0.44836409 & 0.16736190\end{array}$

$1-2.990337821 .33169018-0.27637313$

$\begin{array}{lllll}1 & -3.46256551 & 0.61658084 & 1.24914147\end{array}$

$\begin{array}{lllll}6 & -4.89822377 & 0.34514992 & -0.32944002\end{array}$

$1 \quad-5.36663554-0.540531030 .10858492$

$\begin{array}{llll}1 & -4.89146012 & 0.18238705 & -1.41077813\end{array}$

$\begin{array}{lllll}6 & -5.73166264 & 1.57787606 & -0.00428343\end{array}$

$\begin{array}{llll}1 & -5.29276818 & 2.47360499 & -0.44762866\end{array}$

$\begin{array}{lllll}1 & -5.79097287 & 1.73825876 & 1.07367405\end{array}$

$1-6.749162361 .48260457-0.38400636$ energy $\quad 0.927915$

Symmetry point group: C1

moment of inertia 2.2365

Cartesian coordinate of structure $\quad 60$

$64.199940952 .08358117-0.33576250$

$14.626125112 .73480333-1.09893459$ 
$13.131199601 .99738734-0.53745076$

$14.31427305 \quad 2.580407300 .62987849$

$\begin{array}{lllll}6 & 4.88195054 & 0.72039322 & -0.33259263\end{array}$

$15.96157850 \quad 0.85813378-0.24226867$

$1 \quad 4.721875920 .23251103-1.29693547$

$\begin{array}{lllll}6 & 4.41698053 & -0.20097072 & 0.79636073\end{array}$

$\begin{array}{lllll}1 & 4.71011511 & 0.24035652 & 1.75322553\end{array}$

$\begin{array}{lllll}1 & 4.95428452 & -1.15038590 & 0.71849328\end{array}$

$\begin{array}{lllll}6 & 2.91498092 & -0.48245457 & 0.82685577\end{array}$

$\begin{array}{lllll}1 & 2.37558825 & 0.44564770 & 1.02851060\end{array}$

$1 \quad 2.70029892-1.143737801 .67134496$

$62.38006904-1.12502394-0.44952486$

$12.99969576-1.99331546-0.69233206$

$12.48910013-0.42954453-1.28706632$

$6 \quad 0.92003632-1.56266720-0.36167269$

$1 \quad 0.80377666-2.27363140 \quad 0.46279137$

$10.65750905-2.10740551-1.27285446$

$6-0.06735819-0.41517795-0.17674586$

$\begin{array}{lllll}1 & 0.09322528 & 0.32761799 & -0.96551489\end{array}$

$\begin{array}{lllll}1 & 0.12857325 & 0.09723195 & 0.76887277\end{array}$

$\begin{array}{llll}6 & -1.52160521 & -0.86859700 & -0.20018046\end{array}$

$\begin{array}{llll}1 & -1.67766932 & -1.62030797 & 0.58024019\end{array}$

$1-1.72883062-1.37083888-1.15054419$

$\begin{array}{llll}6 & -2.51384052 & 0.27093193 & -0.00631640\end{array}$

$\begin{array}{lllll}1 & -2.35564284 & 1.02411041 & -0.78489032\end{array}$

$\begin{array}{lllll}1 & -2.30830126 & 0.77105294 & 0.94555121\end{array}$

$6-3.96803420-0.18183856-0.03269424$

$1-4.12665274-0.935368120 .74560114$

$1-4.17432910-0.68177111-0.98464748$

$\begin{array}{lllll}6 & -4.96163527 & 0.95723959 & 0.16158817\end{array}$

$\begin{array}{lllll}1 & -4.80221966 & 1.70893788 & -0.61645621\end{array}$

$\begin{array}{lllll}1 & -4.75462779 & 1.45547948 & 1.11275906\end{array}$

$\begin{array}{lllll}6 & -6.41184154 & 0.49254774 & 0.13338866\end{array}$

$\begin{array}{lllll}1 & -6.60336687 & -0.23750859 & 0.92193168\end{array}$

$1-6.651378260 .01787037-0.81991270$

$\begin{array}{lllll}1 & -7.10206088 & 1.32448518 & 0.27438567\end{array}$ energy $\quad 0.912239$

Symmetry point group: C1 moment of inertia 2.0592

Cartesian coordinate of structure 61

$61.942344632 .57222459-0.00089359$

$1 \quad 1.702948913 .42268373-0.63921306$

$1 \quad 1.110112621 .86976839-0.06627237$

$1 \quad 1.991215452 .929976861 .02961975$

$6 \quad 3.257649041 .92429836-0.41772446$

$14.038865972 .68668800-0.45200199$

$13.166518341 .54248114-1.43722730$

$\begin{array}{llll}6 & 3.71857271 & 0.80321705 & 0.51523139\end{array}$ 


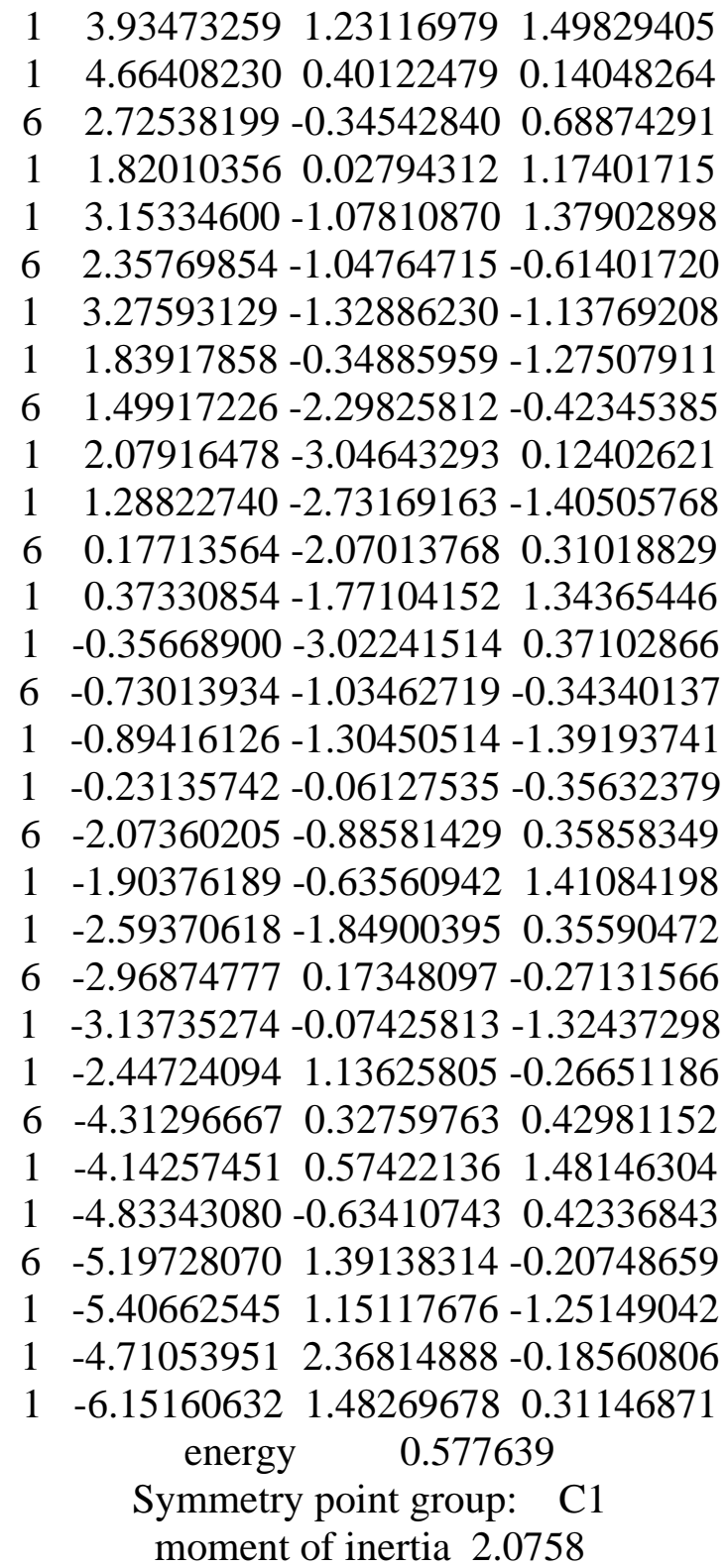

Cartesian coordinate of structure 62

6 -1.86317106 -2.51806181 -0.51810943

$1-1.72069150-3.20500896-1.35235385$

$1-1.06552480-1.77551852-0.56598093$

$1-1.73171265-3.082709760 .40718559$

$6-3.24024731-1.86683508-0.57023927$

$1-4.00269165-2.64533354-0.64410175$

$1-3.32754598-1.27525417-1.48450185$

$\begin{array}{lllll}6 & -3.55955830 & -0.99472242 & 0.64490308\end{array}$

$1-3.59593146-1.632247641 .53293188$

$\begin{array}{llll}1 & -4.56429175 & -0.57948979 & 0.52432291\end{array}$

$\begin{array}{lllll}6 & -2.58009755 & 0.14914275 & 0.90635938\end{array}$

$\begin{array}{lllll}1 & -1.59284373 & -0.25495955 & 1.13756018\end{array}$

$\begin{array}{lllll}1 & -2.90105978 & 0.67960324 & 1.80693870\end{array}$

$\begin{array}{lllll}6 & -2.48103760 & 1.15695192 & -0.23708345\end{array}$ 


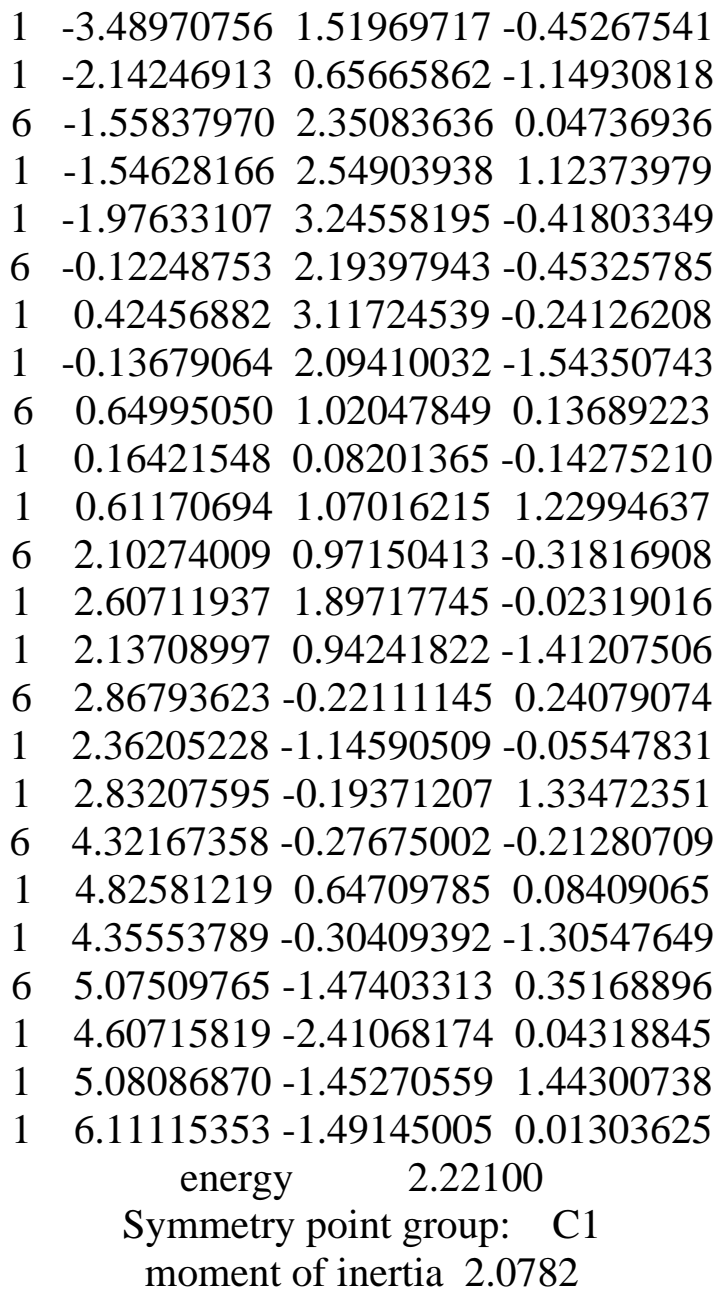

Cartesian coordinate of structure 63

$\begin{array}{llll}6 & -5.53361129 & -0.62847258 & 0.98374339\end{array}$

$1-6.41566775-1.254530940 .84886057$

$1-4.69231758-1.288817541 .19872704$

$\begin{array}{llll}1 & -5.69721739 & -0.00528952 & 1.86536928\end{array}$

$\begin{array}{llll}6 & -5.27331818 & 0.22843666 & -0.24954320\end{array}$

$1-6.177801010 .78900050-0.49547124$

$1-5.08110459-0.41934216-1.10792655$

$\begin{array}{lllll}6 & -4.12313992 & 1.22236554 & -0.08073025\end{array}$

$\begin{array}{lllll}1 & -4.39870093 & 1.94892176 & 0.68901930\end{array}$

$\begin{array}{lllll}1 & -4.00984838 & 1.78882451 & -1.00941052\end{array}$

$\begin{array}{lllll}6 & -2.77238687 & 0.60954524 & 0.29069188\end{array}$

$\begin{array}{lllll}1 & -2.83687994 & 0.13441125 & 1.27413795\end{array}$

$\begin{array}{lllll}1 & -2.05077227 & 1.42190170 & 0.39766294\end{array}$

$6-2.25121606-0.40844881-0.71891061$

$1-2.194455730 .06064521-1.70705346$

$1-2.97072073-1.22540060-0.81128057$

$6-0.88990674-1.00223410-0.36230167$

$1-0.66422377-1.81270424-1.06096429$

$1-0.94712930-1.463489930 .62915689$

$6 \quad 0.26387421-0.00531508-0.38522962$ 
$\begin{array}{llll}1 & 0.09729544 & 0.77870346 & 0.35804662\end{array}$

$1 \quad 0.28622031 \quad 0.49617782-1.35849191$

$61.61706346-0.65400126-0.12160669$

$1 \quad 1.79772577-1.43150933-0.87076671$

$1 \quad 1.58954086-1.165830590 .84575501$

$\begin{array}{lllll}6 & 2.77522888 & 0.33550453 & -0.13405101\end{array}$

$\begin{array}{lllll}1 & 2.59641830 & 1.11066621 & 0.61799568\end{array}$

$1 \quad 2.800295210 .85020089-1.09994350$

$\begin{array}{lllll}6 & 4.12869584 & -0.31364311 & 0.12505576\end{array}$

$14.30837655-1.08855921-0.62721868$

$14.10407912-0.82904607 \quad 1.09070423$

$\begin{array}{lllll}6 & 5.28801106 & 0.67545413 & 0.11343418\end{array}$

$1 \quad 5.10770274 \quad 1.448363090 .86571098$

$15.311106501 .18968819-0.85138136$

$\begin{array}{llll}6 & 6.63581400 & 0.01508353 & 0.37259855\end{array}$

$16.85212694-0.74110733-0.38427057$

$1 \quad 6.64761327-0.479990541 .34534358$

$\begin{array}{lllll}1 & 7.44768793 & 0.74246524 & 0.35878508\end{array}$ energy $\quad 1.10052$

Symmetry point group: C1 moment of inertia 2.1994

Cartesian coordinate of structure 64

$6 \quad 5.24501534-0.729648990 .38649430$

$16.24605035-0.62902303-0.03302433$

$\begin{array}{lllll}1 & 4.95729181 & 0.24390181 & 0.78586687\end{array}$

$15.30227490-1.426209521 .22544130$

$6 \quad 4.25508154-1.22045413-0.66341494$

$14.63495592-2.14021432-1.11340362$

$14.19556804-0.49193370-1.47546284$

$62.85548953-1.49540782-0.11164356$

$12.91700734-2.314353460 .61077368$

$12.22091365-1.85354451-0.92756740$

$\begin{array}{lllll}6 & 2.16993351 & -0.30396014 & 0.55574537\end{array}$

$1 \quad 2.72363125-0.008140901 .45137190$

$1 \quad 1.18829036-0.624089350 .90880982$

$6 \quad 2.018079260 .91590815-0.35118886$

$1 \quad 1.59666340 \quad 0.60367617-1.31301478$

$13.011368041 .30969341-0.57604210$

$\begin{array}{lllll}6 & 1.15345762 & 2.03553355 & 0.24534364\end{array}$

$1 \quad 1.567130343 .00464809-0.03987031$

$\begin{array}{lllll}1 & 1.21316831 & 1.99220718 & 1.33746016\end{array}$

$\begin{array}{llll}6 & -0.31404319 & 2.01117504-0.18263835\end{array}$

$1-0.367443992 .15432372-1.26684436$

$\begin{array}{lllll}1 & -0.82288391 & 2.87161749 & 0.26154754\end{array}$

$\begin{array}{lllll}6 & -1.08181318 & 0.74618670 & 0.18304520\end{array}$

$\begin{array}{lllll}1 & -0.98845069 & 0.56075628 & 1.25812615\end{array}$

$1-0.63315946-0.11713471-0.31655823$

$\begin{array}{lllll}6 & -2.55707149 & 0.81795703 & -0.19058281\end{array}$ 
$1-2.648142231 .01574205-1.26353323$

$\begin{array}{llll}1 & -3.01775923 & 1.67239953 & 0.31542956\end{array}$

$\begin{array}{lllll}6 & -3.32966738 & -0.44855101 & 0.15482689\end{array}$

$\begin{array}{llll}1 & -3.23814223 & -0.64711121 & 1.22764562\end{array}$

$1-2.86883402-1.30291842-0.35157958$

$6-4.80546958-0.38004544-0.21896906$

$1-4.89508301-0.18208220-1.29070185$

$\begin{array}{lllll}1 & -5.26464345 & 0.47350854 & 0.28739326\end{array}$

$\begin{array}{llll}6 & -5.56706819-1.65176802 & 0.13104185\end{array}$

$\begin{array}{lllll}1 & -5.51815323 & -1.85394071 & 1.20257808\end{array}$

$1-5.14451155-2.51497101-0.38644930$

$1-6.61864956-1.57835687-0.14675010$

energy $\quad 2.71624$

Symmetry point group: C1 moment of inertia 2.1023

Cartesian coordinate of structure 65

$6-5.36362262-0.34060423-0.26690577$

$\begin{array}{llll}1 & -6.32409858 & -0.30051912 & 0.24693389\end{array}$

$\begin{array}{lllll}1 & -4.97901882 & 0.67867911 & -0.32353532\end{array}$

$1-5.54445582-0.68197216-1.28825808$

$\begin{array}{lllll}6 & -4.39197050 & -1.26728553 & 0.45433710\end{array}$

$\begin{array}{lllll}1 & -4.86023293 & -2.24455801 & 0.59016399\end{array}$

$\begin{array}{lllll}1 & -4.20336360 & -0.88411785 & 1.45982027\end{array}$

$6-3.06667592-1.46738129-0.28222879$

$1-3.26522477-1.98236124-1.22652308$

$1-2.43620392-2.14002000 \quad 0.30635112$

$6-2.27992428-0.19076402-0.58021694$

$1-2.847779520 .44753012-1.26379092$

$1-1.37260471-0.47203677-1.11976107$

$\begin{array}{lllll}6 & -1.90554007 & 0.61279275 & 0.66021125\end{array}$

$\begin{array}{llll}1 & -1.34064033 & -0.02693143 & 1.34478100\end{array}$

$\begin{array}{lllll}1 & -2.81277205 & 0.89737204 & 1.19811071\end{array}$

$\begin{array}{lllll}6 & -1.11318606 & 1.88643454 & 0.36281896\end{array}$

$\begin{array}{lllll}1 & -0.90560462 & 2.39576663 & 1.30805193\end{array}$

$1-1.743608952 .56575208-0.21795494$

$6 \quad 0.204639451 .68214047-0.38474666$

$1 \quad 0.683762602 .65644397-0.51440174$

$1 \quad 0.007592391 .31095777-1.39430046$

$\begin{array}{lllll}6 & 1.18000633 & 0.74029203 & 0.31132361\end{array}$

$1 \quad 0.74408303-0.260660130 .37996870$

$\begin{array}{lllll}1 & 1.33465983 & 1.07537847 & 1.34240482\end{array}$

$\begin{array}{lllll}6 & 2.52542718 & 0.64347031 & -0.39659568\end{array}$

$12.976052211 .63932063-0.45605538$

$1 \quad 2.365930680 .32079917-1.43059956$

$\begin{array}{llll}6 & 3.49931011 & -0.31035237 & 0.28307871\end{array}$

$13.04899215-1.306635640 .34133118$

$1 \quad 3.658186460 .010996841 .31760396$

$64.84550272-0.40891209-0.42435976$ 


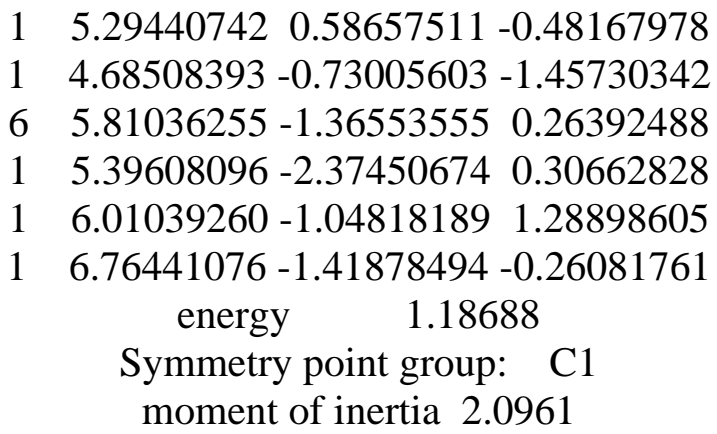

Cartesian coordinate of structure 66

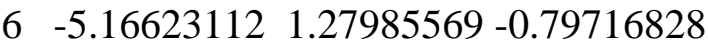

$\begin{array}{llll}1 & -5.29699313 & 2.26648914 & -1.24175941\end{array}$

$1-4.856688400 .59991224-1.59192023$

$1-6.140558950 .94381116-0.43654431$

$\begin{array}{lllll}6 & -4.14920681 & 1.31761309 & 0.33762748\end{array}$

$\begin{array}{lllll}1 & -4.44299720 & 2.08382908 & 1.05825956\end{array}$

$\begin{array}{lllll}1 & -3.17883170 & 1.63241117 & -0.05519588\end{array}$

$\begin{array}{lllll}6 & -3.99962237 & -0.01102752 & 1.07853403\end{array}$

$\begin{array}{llll}1 & -4.95265882 & -0.25885913 & 1.55429753\end{array}$

$\begin{array}{lllll}1 & -3.28228342 & 0.11855618 & 1.89224885\end{array}$

$\begin{array}{lllll}6 & -3.57336219 & -1.19539961 & 0.20757565\end{array}$

$1-4.37866319-1.44253454-0.48815990$

$1-3.45405419-2.071111820 .85145682$

$6-2.28477902-0.98637407-0.58663618$

$1-2.42247115-0.17982830-1.31314274$

$1-2.08852673-1.88687066-1.17500475$

$\begin{array}{lllll}6 & -1.06165218 & -0.68103124 & 0.27024610\end{array}$

$\begin{array}{llll}1 & -0.94881673-1.46105962 & 1.03053066\end{array}$

$\begin{array}{lllll}1 & -1.21371912 & 0.25461923 & 0.81582403\end{array}$

$6 \quad 0.22375995-0.57531714-0.54043374$

$1 \quad 0.103521190 .19658492-1.30749227$

$1 \quad 0.39080609-1.51384552-1.07855591$

$\begin{array}{lllll}6 & 1.44875442 & -0.25565964 & 0.30670709\end{array}$

$1 \quad 1.56708508-1.025650361 .07579631$

$\begin{array}{lllll}1 & 1.28233577 & 0.68456522 & 0.84213152\end{array}$

$6 \quad 2.73478814-0.15215298-0.50333414$

$1 \quad 2.616123780 .61733606-1.27290657$

$12.90158384-1.09266354-1.03815815$

$\begin{array}{lllll}6 & 3.95942286 & 0.16851312 & 0.34392157\end{array}$

$14.07800681-0.600314031 .11434089$

$1 \quad 3.79357850 \quad 1.10960376 \quad 0.87829611$

$\begin{array}{lllll}6 & 5.24684421 & 0.27088365 & -0.46513971\end{array}$

$15.127084201 .03895295-1.23425261$

$15.41137402-0.66985659-0.99782676$

$\begin{array}{lllll}6 & 6.46405402 & 0.59191987 & 0.39228785\end{array}$

$1 \quad 6.62150595-0.177386221 .15046309$

$\begin{array}{lllll}1 & 6.33646836 & 1.54393380 & 0.91079370\end{array}$

$17.371169720 .65843602-0.20864595$ 


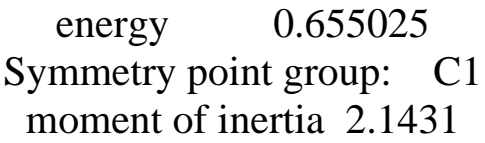

Cartesian coordinate of structure 67

$\begin{array}{llll}6 & 3.67791222 & 2.38109555 & 0.39094599\end{array}$

$\begin{array}{llll}1 & 3.31013881 & 3.38239302 & 0.16658325\end{array}$

$1 \quad 3.195456352 .052913331 .31254647$

14.749402992 .454662690 .58790765

$6 \quad 3.40067309 \quad 1.42175385-0.76068304$

$13.812732231 .83805021-1.68242035$

$1 \quad 2.32162697 \quad 1.34938807-0.92085466$

$6 \quad 3.986861640 .02473502-0.55661494$

$15.07633030 \quad 0.10323142-0.50291865$

$13.77218156-0.57929172-1.44121838$

$6 \quad 3.49363511-0.709353790 .69303723$

$13.87442356-0.204801381 .58413522$

$13.93483463-1.70985156 \quad 0.70220686$

$\begin{array}{llll}6 & 1.97522786 & -0.83432397 & 0.80979411\end{array}$

$\begin{array}{llll}1 & 1.53594958 & 0.16132678 & 0.90741202\end{array}$

$1 \quad 1.73661099-1.35992267 \quad 1.73892077$

$6 \quad 1.32344219-1.57429971-0.35456117$

$1 \quad 1.83168389-2.53366997-0.48884415$

$1 \quad 1.47895132-1.01515109-1.28188297$

$6 \quad-0.17248826-1.82172664-0.17609754$

$1-0.33903800-2.392418970 .74329649$

$1-0.52758246-2.45330505-0.99519252$

$6-1.01596847-0.55160545-0.13769602$

$\begin{array}{lllll}1 & -0.79814044 & 0.05099362 & -1.02602465\end{array}$

$\begin{array}{lllll}1 & -0.73136590 & 0.05998550 & 0.72255676\end{array}$

$6-2.51248729-0.82924352-0.07184991$

$\begin{array}{lllll}1 & -2.72715410 & -1.44349153 & 0.80858418\end{array}$

$1-2.80776553-1.42863779-0.93900154$

$\begin{array}{llll}6 & -3.36043377 & 0.43516863 & -0.02166620\end{array}$

$1-3.14265644 \quad 1.05206032-0.89961598$

$\begin{array}{lllll}1 & -3.06846099 & 1.03259550 & 0.84811739\end{array}$

$\begin{array}{lllll}6 & -4.85797544 & 0.15924630 & 0.03888606\end{array}$

$1-5.07389845-0.45763500 \quad 0.91562224$

$1-5.14839159-0.43635557-0.83100435$

$\begin{array}{lllll}6 & -5.69526505 & 1.43052651 & 0.09008710\end{array}$

$\begin{array}{lllll}1 & -5.51878861 & 2.05016203 & -0.79104457\end{array}$

$\begin{array}{lllll}1 & -5.44453289 & 2.02818464 & 0.96837878\end{array}$

$\begin{array}{lllll}1 & -6.76135079 & 1.20674858 & 0.13226461\end{array}$ energy $\quad 0.934434$

Symmetry point group: C1 moment of inertia 2.0222

Cartesian coordinate of structure $\quad 68$ $64.915726291 .66966969-0.29150940$ 
$1 \quad 5.206029092 .31077359-1.12392492$

$\begin{array}{lllll}1 & 4.09922944 & 2.16506373 & 0.23546798\end{array}$

$1 \quad 5.762606751 .609168830 .39508763$

$\begin{array}{lllll}6 & 4.51044529 & 0.28357821 & -0.77933324\end{array}$

$15.31634906-0.13335234-1.38711211$

$13.647850220 .37088013-1.44516221$

$\begin{array}{lllll}6 & 4.19503939 & -0.70054791 & 0.34687931\end{array}$

$15.09899195-0.857130320 .94222218$

$13.95326848-1.67143984-0.09151461$

$6 \quad 3.06796425-0.26915567 \quad 1.28873139$

$1 \quad 3.395130640 .594862191 .87190915$

$1 \quad 2.89725041-1.071184222 .01216679$

$\begin{array}{lllll}6 & 1.74228631 & 0.07396670 & 0.60925359\end{array}$

$1 \quad 1.873869330 .93889978-0.04857245$

$\begin{array}{lllll}1 & 1.03747036 & 0.38957877 & 1.38104705\end{array}$

$6 \quad 1.13947641-1.07297987-0.19610131$

$1 \quad 0.99466205-1.93669600 \quad 0.46162073$

$1 \quad 1.85163343-1.38982421-0.96153548$

$6-0.18261920-0.73000072-0.87946698$

$1-0.47068294-1.56169792-1.52848516$

$\begin{array}{lllll}1 & -0.03365502 & 0.13166293 & -1.53858387\end{array}$

$\begin{array}{lllll}6 & -1.33458162 & -0.43638165 & 0.07565225\end{array}$

$\begin{array}{lllll}1 & -1.10155679 & 0.44014109 & 0.68580295\end{array}$

$\begin{array}{llll}1 & -1.44575184 & -1.27329900 & 0.77312322\end{array}$

$6-2.65763605-0.19892703-0.64171050$

$1-2.90648997-1.07921378-1.24305066$

$1-2.540863010 .62829646-1.34928790$

$\begin{array}{lllll}6 & -3.81203982 & 0.10749811 & 0.30364193\end{array}$

$\begin{array}{lllll}1 & -3.56487688 & 0.99002844 & 0.90260597\end{array}$

$1-3.92733866-0.717799571 .01382094$

$\begin{array}{lllll}6 & -5.13661747 & 0.34214171 & -0.41253316\end{array}$

$1-5.38315862-0.54051576-1.00913426$

$1-5.019589661 .16585375-1.12220994$

$\begin{array}{lllll}6 & -6.28265417 & 0.64957537 & 0.54240309\end{array}$

$\begin{array}{lllll}1 & -6.07260526 & 1.54602041 & 1.12870145\end{array}$

$\begin{array}{llll}1 & -6.43867807 & -0.17283411 & 1.24295881\end{array}$

$\begin{array}{lllll}1 & -7.21783219 & 0.81313531 & 0.00659702\end{array}$ energy $\quad 1.22870$

Symmetry point group: C1 moment of inertia 2.0896

Cartesian coordinate of structure 69

$65.49821019-0.63064587-0.28993024$

$1 \quad 6.16535196-0.86298261-1.12007414$

$15.485151050 .45377692-0.17298359$

$15.93289996-1.055147340 .61734253$

$64.10009848-1.18594612-0.53564556$

$14.17065825-2.25400638-0.75247443$

$13.67979926-0.72485029-1.43301565$ 


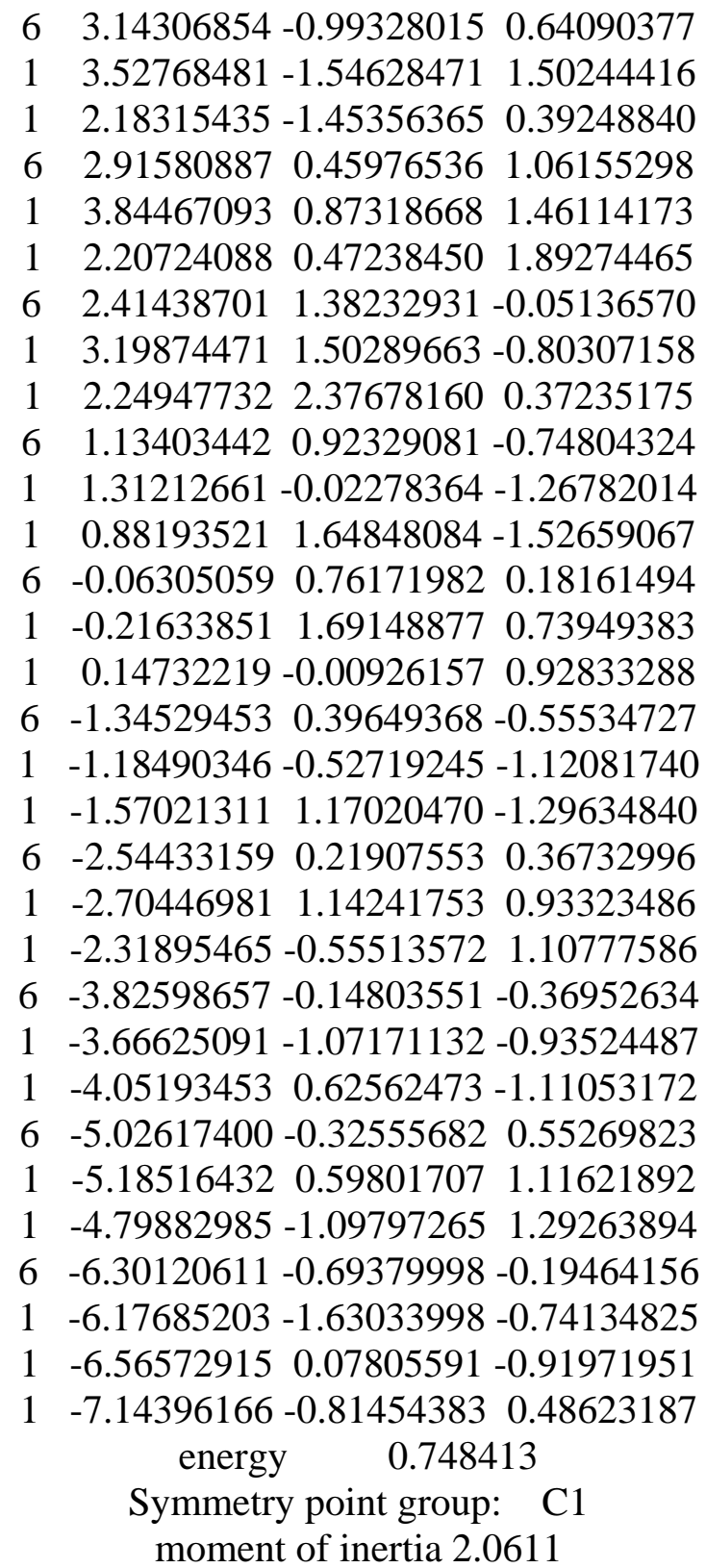

Cartesian coordinate of structure $\quad 70$

6 -5.27983179 $-0.87755290-0.39349228$

$1-6.26090039-0.44413959-0.58802224$

$1-4.77649015-0.99485699-1.35404263$

$\begin{array}{lllll}1 & -5.43172522 & -1.87523560 & 0.02349082\end{array}$

$\begin{array}{llll}6 & -4.47241714 & -0.00545377 & 0.56092836\end{array}$

$\begin{array}{llll}1 & -5.05758410 & 0.17465068 & 1.46529524\end{array}$

$\begin{array}{lllll}1 & -4.31229507 & 0.97597468 & 0.10744370\end{array}$

$\begin{array}{llll}6 & -3.12946490 & -0.61257685 & 0.96737011\end{array}$

$1-3.31393645-1.54237667 \quad 1.51277611$

$1-2.640189650 .060287151 .67612390$

$6-2.17578592-0.91267258-0.19064260$

$1-2.59876510-1.70247862-0.81562259$

$\begin{array}{llll}1 & -1.25205913 & -1.32695288 & 0.22129155\end{array}$ 


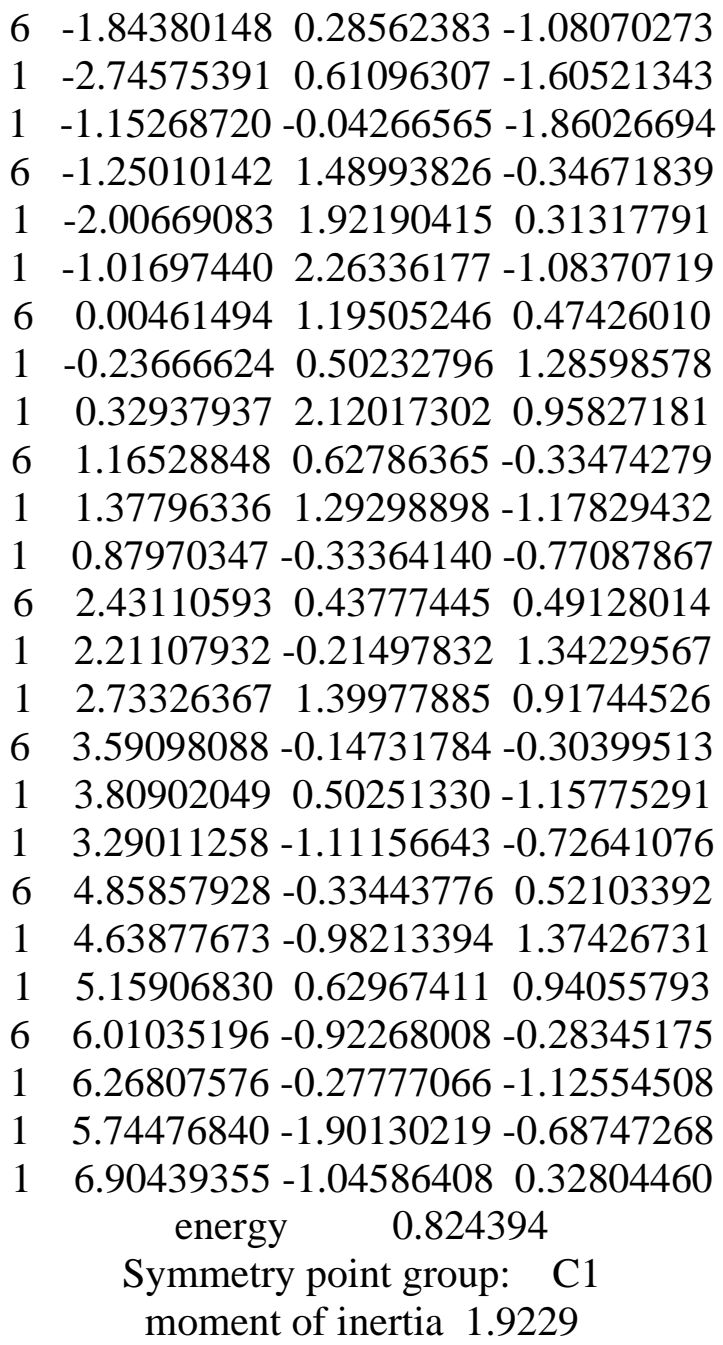

Cartesian coordinate of structure $\quad 71$

$6 \quad 4.82857472-1.392768800 .05608757$

$15.71301688-1.48755923-0.57400016$

$14.98423642-0.536162770 .71321807$

$1 \quad 4.76863311-2.283737110 .68449034$

$63.56768902-1.23426237-0.78555607$

$13.50749630-2.05759504-1.50054952$

$13.64286353-0.32289356-1.38417242$

$\begin{array}{llll}6 & 2.27551261 & -1.20996082 & 0.03119139\end{array}$

$1 \quad 2.16074896-2.172041050 .53864234$

$1 \quad 1.43025158-1.12928840-0.65589766$

$6 \quad 2.19054946-0.098795761 .07913669$

$12.94011165-0.275917951 .85425533$

$1 \quad 1.22599506-0.166732481 .58709755$

$\begin{array}{llll}6 & 2.38885008 & 1.31807970 & 0.53974865\end{array}$

$\begin{array}{lllll}1 & 3.41123482 & 1.41450384 & 0.16447542\end{array}$

$1 \quad 2.312886692 .018772141 .37595662$

$6 \quad 1.429335031 .77623270-0.56125546$

$11.513279661 .11737120-1.43061903$

$1 \quad 1.773595942 .75577614-0.89938935$ 
$\begin{array}{lllll}6 & -0.04442372 & 1.89237551 & -0.15280672\end{array}$

$1-0.513218462 .68089501-0.74770416$

$\begin{array}{lllll}1 & -0.10574455 & 2.22753726 & 0.88792224\end{array}$

$\begin{array}{lllll}6 & -0.87819452 & 0.62567379 & -0.32575391\end{array}$

$\begin{array}{lllll}1 & -0.47584477 & -0.18374457 & 0.28636929\end{array}$

$\begin{array}{lllll}1 & -0.80045340 & 0.28759261 & -1.36423492\end{array}$

$\begin{array}{lllll}6 & -2.34614062 & 0.82818745 & 0.02749743\end{array}$

$\begin{array}{lllll}1 & -2.76074822 & 1.63034242 & -0.59157478\end{array}$

$\begin{array}{lllll}1 & -2.42379864 & 1.17375303 & 1.06343675\end{array}$

$6-3.18755465-0.42954886-0.14724318$

$1-2.77406694-1.230720070 .47394210$

$1-3.10787159-0.77734925-1.18233380$

$\begin{array}{lllll}6 & -4.65710681 & -0.23022564 & 0.20353256\end{array}$

$1-5.069077860 .56928085-0.41845189$

$\begin{array}{lllll}1 & -4.73510293 & 0.11779437 & 1.23727158\end{array}$

$\begin{array}{llll}6 & -5.48746345 & -1.49470487 & 0.02620453\end{array}$

$1-5.11356540-2.29978750 \quad 0.66142248$

1 -5.44903127 -1.84549191-1.00672515

$\begin{array}{lllll}1 & -6.53358947 & -1.32629014 & 0.28245175\end{array}$

energy $\quad 2.41048$

Symmetry point group: C1

moment of inertia 1.9608

Cartesian coordinate of structure $\quad 72$

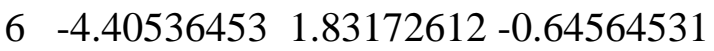

$\begin{array}{llll}1 & -4.35652221 & 2.69328804 & -1.31159508\end{array}$

$\begin{array}{lllll}1 & -4.68005479 & 0.96580978 & -1.24930417\end{array}$

$\begin{array}{lllll}1 & -5.21302410 & 2.00614974 & 0.06826229\end{array}$

$\begin{array}{lllll}6 & -3.07805968 & 1.61674603 & 0.07258767\end{array}$

$\begin{array}{lllll}1 & -2.78301327 & 2.54408726 & 0.56855948\end{array}$

$\begin{array}{lllll}1 & -2.29897650 & 1.40981663 & -0.66448931\end{array}$

$\begin{array}{lllll}6 & -3.11500298 & 0.50131299 & 1.11721688\end{array}$

$\begin{array}{llll}1 & -3.83026133 & 0.77796023 & 1.89733561\end{array}$

$\begin{array}{lllll}1 & -2.14326820 & 0.44125884 & 1.61274127\end{array}$

$\begin{array}{lllll}6 & -3.50430281 & -0.87838012 & 0.58437899\end{array}$

$\begin{array}{lllll}1 & -4.53692621 & -0.84276790 & 0.22826265\end{array}$

$1-3.50339291-1.582430461 .42121553$

$6-2.63036776-1.45559838-0.53161279$

$1-2.65059436-0.79560238-1.40408875$

$1-3.09896379-2.38691423-0.85626196$

$6-1.17403618-1.74964302-0.15040920$

$1-0.81830271-2.59537583-0.74502630$

$\begin{array}{lllll}1 & -1.13347097 & -2.08043037 & 0.89274169\end{array}$

$6-0.19393572-0.59731043-0.35352065$

$\begin{array}{lllll}1 & -0.48241729 & 0.26221646 & 0.25462130\end{array}$

$1-0.24822670-0.26355402-1.39490452$

$6 \quad 1.24397175-0.97566909-0.02205357$

$11.54401038-1.83213342-0.63441595$ 
$1 \quad 1.29782054-1.313055251 .01806265$

$\begin{array}{lllll}6 & 2.23169072 & 0.16489851 & -0.23246638\end{array}$

$\begin{array}{lllll}1 & 1.93111074 & 1.02083377 & 0.38028886\end{array}$

$1 \quad 2.177063230 .50258528-1.27238912$

$6 \quad 3.67012990-0.210643820 .09941552$

$13.97098816-1.06731509-0.51237750$

$13.72558090-0.547181601 .13978790$

$64.658857090 .92948974-0.11272864$

$\begin{array}{lllll}1 & 4.35711514 & 1.78443697 & 0.49869983\end{array}$

$14.602644091 .26414952-1.15227710$

$\begin{array}{lllll}6 & 6.09338355 & 0.54273247 & 0.22248564\end{array}$

$16.42798642-0.29119655-0.39724594$

$\begin{array}{lllll}1 & 6.18095538 & 0.23352508 & 1.26564168\end{array}$

$\begin{array}{lllll}1 & 6.78036021 & 1.37387351 & 0.06226593\end{array}$ energy $\quad 2.33799$

Symmetry point group: C1 moment of inertia 2.0406

Cartesian coordinate of structure 73

$6-2.412074952 .65311019-0.57292751$

$\begin{array}{lllll}1 & -1.66242821 & 3.42032181 & -0.76680283\end{array}$

$1-2.587681992 .12136123-1.50896240$

$1-3.342120353 .15567359-0.29957593$

$\begin{array}{lllll}6 & -1.95695148 & 1.70719892 & 0.53229916\end{array}$

$\begin{array}{lllll}1 & -1.68563218 & 2.28958430 & 1.41558539\end{array}$

$\begin{array}{lllll}1 & -1.04284513 & 1.19905926 & 0.21677548\end{array}$

$\begin{array}{lllll}6 & -3.01124505 & 0.67740176 & 0.93802203\end{array}$

$\begin{array}{lllll}1 & -3.87318870 & 1.20690289 & 1.35452063\end{array}$

$\begin{array}{lllll}1 & -2.61826772 & 0.06446476 & 1.75182678\end{array}$

$6-3.51286409-0.22554536-0.19093316$

$\begin{array}{lllll}1 & -4.07558534 & 0.38232124 & -0.90403139\end{array}$

$\begin{array}{lllll}1 & -4.23391872 & -0.93203654 & 0.22983988\end{array}$

$6-2.45770062-1.01499814-0.96998215$

$1-1.75693332-0.32629765-1.44803665$

$1-2.97564443-1.51575081-1.79068826$

$6-1.69165122-2.07835968-0.17207677$

$1-1.36356331-2.86117024-0.86173356$

$\begin{array}{llll}1 & -2.38663480 & -2.56185325 & 0.52062013\end{array}$

$\begin{array}{llll}6 & -0.46166437 & -1.60265388 & 0.60463117\end{array}$

$1-0.09660238-2.433174051 .21536985$

$\begin{array}{llll}1 & -0.73416122 & -0.81355385 & 1.30763497\end{array}$

$6 \quad 0.68238419-1.11151520-0.27510299$

$1 \quad 0.33593512-0.30653375-0.92953342$

$11.00257633-1.92206104-0.93771786$

$\begin{array}{lllll}6 & 1.87484481 & -0.60814891 & 0.52787473\end{array}$

$1 \quad 2.23367246-1.406413001 .18542932$

$\begin{array}{lllll}1 & 1.54585931 & 0.20251579 & 1.18646341\end{array}$

$63.02443682-0.11416555-0.34054886$

$12.663679020 .68353476-0.99809966$ 
$13.35480181-0.92367690-0.99948914$

$\begin{array}{lllll}6 & 4.21450040 & 0.39726638 & 0.46228007\end{array}$

$14.57426206-0.400379751 .11811680$

$1 \quad 3.882503241 .205328851 .12002518$

$6 \quad 5.356708550 .89049504-0.41628284$

$15.029164061 .70935162-1.05943812$

$1 \quad 5.725539060 .09174671-1.06237501$

$\begin{array}{lllll}1 & 6.19487761 & 1.25022059 & 0.18075921\end{array}$ energy $\quad 2.26155$

Symmetry point group: C1 moment of inertia 1.9779

Cartesian coordinate of structure $\quad 74$

$\begin{array}{llll}6 & -3.82584847 & 2.11275684 & -0.44246386\end{array}$

$1-3.477999583 .10322946-0.14828201$

$1-3.105060101 .71085323-1.15543342$

$\begin{array}{lllll}1 & -4.77605911 & 2.23459515 & -0.96635064\end{array}$

$\begin{array}{lllll}6 & -3.99058228 & 1.20420541 & 0.76977173\end{array}$

$\begin{array}{lllll}1 & -4.65905031 & 1.68639171 & 1.48672355\end{array}$

$\begin{array}{lllll}1 & -3.03314901 & 1.09413571 & 1.28399717\end{array}$

$\begin{array}{lllll}6 & -4.56180715 & -0.17406255 & 0.43838514\end{array}$

$1-5.55482473-0.03938235-0.00086864$

$\begin{array}{lllll}1 & -4.71379850 & -0.72159687 & 1.37291429\end{array}$

$6-3.73523801-1.05117735-0.50495523$

$1-3.63559857-0.56522217-1.47953696$

$1-4.31258832-1.95992597-0.68720668$

$\begin{array}{lllll}6 & -2.34504590 & -1.44991644 & 0.00589306\end{array}$

$1-2.09191823-2.43486042-0.39537114$

$\begin{array}{lllll}1 & -2.37910737 & -1.57074160 & 1.09379781\end{array}$

$\begin{array}{llll}6 & -1.21194707-0.49548158-0.36335775\end{array}$

$\begin{array}{lllll}1 & -1.39558209 & 0.49696032 & 0.05332310\end{array}$

$1-1.19307347-0.36885344-1.45067175$

$\begin{array}{lllll}6 & 0.15118989 & -0.98601733 & 0.10785683\end{array}$

$1 \quad 0.34575868-1.97427313-0.32109382$

$1 \quad 0.12934450-1.125527891 .19349091$

$6 \quad 1.29354052-0.04588542-0.25581477$

$\begin{array}{lllll}1 & 1.10532976 & 0.93909225 & 0.18309128\end{array}$

$1 \quad 1.309497980 .10250906-1.34025902$

$\begin{array}{lllll}6 & 2.65627745 & -0.54870838 & 0.20357591\end{array}$

$12.84329147-1.53311974-0.23724261$

$1 \quad 2.63833807-0.700046551 .28763340$

$\begin{array}{lllll}6 & 3.80326413 & 0.38774787 & -0.15448083\end{array}$

$\begin{array}{lllll}1 & 3.61917679 & 1.37216548 & 0.28785038\end{array}$

$13.822842380 .54067005-1.23837864$

$6 \quad 5.16368622-0.122143450 .30611058$

$15.34582766-1.10475869-0.13783190$

$15.14018155-0.276566731 .38841932$

$\begin{array}{llll}6 & 6.30697832 & 0.81881054 & -0.05066922\end{array}$

$\begin{array}{lllll}1 & 6.16186521 & 1.80004760 & 0.40494017\end{array}$ 
$1 \quad 6.37400977 \quad 0.96335869-1.13047116$

$\begin{array}{lllll}1 & 7.26553941 & 0.43009770 & 0.29370740\end{array}$ energy $\quad 2.12306$

Symmetry point group: C1 moment of inertia 2.1096

Cartesian coordinate of structure $\quad 75$

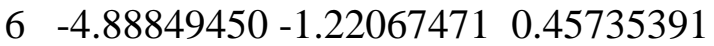

$1-5.10980514-2.257579990 .20379903$

$\begin{array}{lllll}1 & -4.10939643 & -1.22768264 & 1.22037462\end{array}$

$1-5.78452542-0.788001960 .90673633$

$6-4.46650569-0.42969441-0.77519231$

$1-5.25059890-0.50743479-1.53174094$

$1-3.57782189-0.88300059-1.22133974$

$\begin{array}{lllll}6 & -4.21025875 & 1.05179307 & -0.50137873\end{array}$

$\begin{array}{lllll}1 & -5.12811729 & 1.49395342 & -0.10229451\end{array}$

$\begin{array}{lllll}1 & -4.01918859 & 1.55517112 & -1.45338743\end{array}$

$\begin{array}{lllll}6 & -3.05840988 & 1.38065758 & 0.45199142\end{array}$

$\begin{array}{lllll}1 & -3.25568336 & 0.95636009 & 1.44052456\end{array}$

$\begin{array}{lllll}1 & -3.05485521 & 2.46330766 & 0.59215602\end{array}$

$\begin{array}{lllll}6 & -1.66972694 & 0.93356865 & -0.02566825\end{array}$

$\begin{array}{lllll}1 & -0.93241815 & 1.67307621 & 0.29140306\end{array}$

$1-1.644291840 .93482356-1.12092731$

$\begin{array}{lllll}6 & -1.23208849 & -0.43830347 & 0.48552999\end{array}$

$\begin{array}{llll}1 & -1.99819806 & -1.17945458 & 0.25335061\end{array}$

$\begin{array}{lllll}1 & -1.16724559 & -0.40256619 & 1.57802148\end{array}$

$6 \quad 0.10018971-0.92163685-0.08269608$

$1 \quad 0.02540350-0.98110013-1.17354928$

$\begin{array}{lllll}1 & 0.28210453 & -1.94246538 & 0.26490350\end{array}$

$\begin{array}{lllll}6 & 1.30070588 & -0.05804388 & 0.28967033\end{array}$

$\begin{array}{lllll}1 & 1.33765483 & 0.05685279 & 1.37816285\end{array}$

$1 \quad 1.179030330 .94837256-0.11933117$

$62.62341050-0.63265594-0.20189991$

$12.57948378-0.76050017-1.28832972$

$1 \quad 2.76008621-1.635220750 .21598054$

$\begin{array}{lllll}6 & 3.82746228 & 0.22960583 & 0.15445685\end{array}$

$\begin{array}{lllll}1 & 3.87050597 & 0.36012458 & 1.24067559\end{array}$

$13.692324491 .23151021-0.26580830$

$65.15131522-0.34524851-0.33488791$

$15.10655815-0.47548467-1.41972260$

$15.28560803-1.345466810 .08621505$

$\begin{array}{lllll}6 & 6.34750197 & 0.52576620 & 0.02606954\end{array}$

$1 \quad 6.431247840 .64545722 \quad 1.10779726$

$1 \quad 6.250414951 .52220200-0.40885938$

$17.281115580 .09394589-0.33490324$ energy $\quad 2.60128$

Symmetry point group: C1 moment of inertia 2.0314 
Cartesian coordinate of structure $\quad 76$

$\begin{array}{llll}6 & 3.94247752 & 1.42864660 & 1.34079745\end{array}$

13.600626362 .156174762 .07719373

$1 \quad 3.742846030 .43491941 \quad 1.74308462$

$\begin{array}{lllll}1 & 5.02502920 & 1.53370671 & 1.24556092\end{array}$

$6 \quad 3.26062276 \quad 1.64474952-0.00514039$

$13.444377992 .66990988-0.33426055$

$\begin{array}{lllll}1 & 2.17729396 & 1.55868869 & 0.10969414\end{array}$

$\begin{array}{llll}6 & 3.73988389 & 0.69438685-1.10156263\end{array}$

$14.818568740 .82507547-1.22936416$

$13.282636420 .99643451-2.04805065$

$63.44814468-0.79308112-0.88662801$

$13.96402615-1.154179960 .00737905$

$13.89419918-1.33372227-1.72356531$

$6 \quad 1.95777877-1.14916768-0.79563204$

$1 \quad 1.78756060-2.12252029-1.26341971$

$1 \quad 1.38504429-0.42942807-1.38689117$

$6 \quad 1.40664380-1.214697740 .62798356$

$1 \quad 1.58976437-0.271163571 .14720093$

$1 \quad 1.96681216-1.975937031 .17798818$

$\begin{array}{lllll}6 & -0.08405073-1.53438101 & 0.70699696\end{array}$

$\begin{array}{llll}1 & -0.34707589 & -1.72938156 & 1.75038251\end{array}$

$\begin{array}{llll}1 & -0.28896413 & -2.46215566 & 0.16282344\end{array}$

$\begin{array}{lllll}6 & -0.98815529 & -0.42569050 & 0.17746563\end{array}$

$1-0.79571165-0.25853444-0.88554594$

$\begin{array}{lllll}1 & -0.73653151 & 0.51235700 & 0.68380689\end{array}$

$\begin{array}{lllll}6 & -2.47088129 & -0.71686167 & 0.37109628\end{array}$

$\begin{array}{llll}1 & -2.66984550 & -0.88198350 & 1.43480382\end{array}$

$1-2.72372394-1.65465179-0.13392384$

$\begin{array}{llll}6 & -3.37743801 & 0.39374231 & -0.14375105\end{array}$

$1-3.184799890 .55541828-1.20926205$

$\begin{array}{lllll}1 & -3.11866069 & 1.33264473 & 0.35654235\end{array}$

$\begin{array}{lllll}6 & -4.86074333 & 0.11040445 & 0.06081487\end{array}$

$\begin{array}{llll}1 & -5.05068222 & -0.05254631 & 1.12526141\end{array}$

$1-5.11921888-0.82602947-0.44123043$

$\begin{array}{lllll}6 & -5.75612883 & 1.23013706 & -0.45313887\end{array}$

$1-5.605355851 .39088434-1.52215331$

$\begin{array}{lllll}1 & -5.53606482 & 2.17098931 & 0.05477198\end{array}$

$\begin{array}{lllll}1 & -6.81107412 & 1.00590844 & -0.29463747\end{array}$ energy 2.37380

Symmetry point group: C1 moment of inertia 2.0714

Cartesian coordinate of structure $\quad 77$

$\begin{array}{llll}6 & 2.52872379 & 2.23390782 & 0.07423009\end{array}$

$1 \quad 2.201147223 .02992913-0.59465714$

$\begin{array}{lllll}1 & 1.66176118 & 1.60754778 & 0.28796179\end{array}$

$1 \quad 2.840924512 .694240251 .01384300$

$\begin{array}{llll}6 & 3.66848001 & 1.43160448 & -0.54227537\end{array}$ 
$1 \quad 4.472206702 .11552811-0.82468067$

$13.333618150 .96879238-1.47298332$

$\begin{array}{lllll}6 & 4.25537235 & 0.36851419 & 0.38640005\end{array}$

$\begin{array}{lllll}1 & 4.70173693 & 0.87458438 & 1.24781381\end{array}$

$15.07896165-0.13075786-0.13219105$

$6 \quad 3.29459506-0.701882080 .91095138$

$1 \quad 2.51077609-0.23487706 \quad 1.51129407$

$13.85991508-1.323841761 .60812133$

$62.67228662-1.61637320-0.15246801$

$1 \quad 2.45396437-2.585719930 .30424171$

$13.42042371-1.81200438-0.92622706$

$61.38554631-1.11846680-0.81572150$

$11.13083201-1.80170070-1.63084164$

$1 \quad 1.54639577-0.14485521-1.28213222$

$\begin{array}{llll}6 & 0.19056794 & -1.02526386 & 0.12626898\end{array}$

$1 \quad 0.42335562-0.364837500 .96650515$

$1-0.00436381-2.010655720 .56174633$

$6-1.06886744-0.51481376-0.56204413$

$1-1.31349609-1.16995465-1.40422235$

$\begin{array}{lllll}1 & -0.86693775 & 0.47106928 & -0.99361355\end{array}$

$\begin{array}{llll}6 & -2.27118320 & -0.41911660 & 0.36827299\end{array}$

$\begin{array}{lllll}1 & -2.02464784 & 0.23530362 & 1.21056839\end{array}$

$1-2.47422806-1.404710040 .79927124$

$\begin{array}{lllll}6 & -3.52822852 & 0.09827608 & -0.31909212\end{array}$

$1-3.77485135-0.55508948-1.16229084$

$1-3.325561301 .08443177-0.74934842$

$\begin{array}{lllll}6 & -4.73204804 & 0.19400970 & 0.61047345\end{array}$

$\begin{array}{lllll}1 & -4.48424096 & 0.84666635 & 1.45216376\end{array}$

$\begin{array}{lllll}1 & -4.93354086 & -0.79166575 & 1.03902518\end{array}$

$\begin{array}{llll}6 & -5.98243766 & 0.71294978 & -0.08741417\end{array}$

$\begin{array}{llll}1 & -6.26736333 & 0.06027459 & -0.91463761\end{array}$

$1-5.815173721 .71048634-0.49778289$

$\begin{array}{lllll}1 & -6.82845701 & 0.77174160 & 0.59756314\end{array}$ energy $\quad 2.28200$

Symmetry point group: C1 moment of inertia 2.0467

Cartesian coordinate of structure $\quad 78$

$62.743778582 .45737196-0.11882788$

$12.656521613 .13353782-0.96945243$

$\begin{array}{lllll}1 & 1.73274278 & 2.23359931 & 0.22465011\end{array}$

$\begin{array}{llll}1 & 3.25236403 & 2.99365610 & 0.68475922\end{array}$

$6 \quad 3.50844103 \quad 1.19427533-0.49797659$

$14.486602651 .48124117-0.89083998$

$1 \quad 2.993026690 .68950361-1.31601601$

$\begin{array}{lllll}6 & 3.72866438 & 0.23197671 & 0.67251731\end{array}$

$\begin{array}{lllll}1 & 4.24991719 & 0.78651495 & 1.45792038\end{array}$

$1 \quad 4.41492271-0.560333270 .35953097$

$6 \quad 2.47664509-0.416168641 .27873891$ 
$\begin{array}{llll}1 & 1.65033329 & 0.29901845 & 1.29107727\end{array}$

$1 \quad 2.68174370-0.638622352 .32790499$

$\begin{array}{llll}6 & 2.02465378 & -1.72334177 & 0.62313775\end{array}$

$1 \quad 1.22461932-2.151699401 .23197856$

$1 \quad 2.85047170-2.438686240 .67266158$

$6 \quad 1.55007321-1.63747631-0.82874131$

$1 \quad 1.22418997-2.63475697-1.13796629$

$12.39395210-1.38708061-1.47547916$

$6 \quad 0.41362989-0.64938600-1.08661789$

$1 \quad 0.15712804-0.68349902-2.14908688$

$1 \quad 0.75545486 \quad 0.37134107-0.89576168$

$6-0.84449722-0.90783004-0.26600253$

$1-0.61978930-0.807304800 .79964854$

$1-1.16822545-1.94368948-0.41213677$

$\begin{array}{lllll}6 & -1.98735151 & 0.03550441 & -0.61912099\end{array}$

$1-2.22993378-0.07090748-1.68127219$

$1-1.653546041 .06992909-0.48690342$

$\begin{array}{lllll}6 & -3.24349079 & -0.19700341 & 0.21032081\end{array}$

$\begin{array}{llll}1 & -3.00139410 & -0.08689246 & 1.27239253\end{array}$

$\begin{array}{lllll}1 & -3.57625854 & -1.23205728 & 0.08129268\end{array}$

$\begin{array}{lllll}6 & -4.38704249 & 0.74594503 & -0.14408933\end{array}$

$\begin{array}{lllll}1 & -4.62884379 & 0.63374537 & -1.20463223\end{array}$

$\begin{array}{lllll}1 & -4.05254447 & 1.77918218 & -0.01613875\end{array}$

$\begin{array}{lllll}6 & -5.63674616 & 0.50627313 & 0.69290506\end{array}$

$\begin{array}{lllll}1 & -5.42736549 & 0.64300100 & 1.75541518\end{array}$

$\begin{array}{lllll}1 & -6.00748197 & -0.51158026 & 0.55872718\end{array}$

$\begin{array}{lllll}1 & -6.43915449 & 1.19199712 & 0.42026674\end{array}$ energy $\quad 2.46759$

Symmetry point group: C1 moment of inertia 1.9800

Cartesian coordinate of structure $\quad 79$

$\begin{array}{llll}6 & -4.89859873 & 1.86007029 & -0.09664685\end{array}$

$\begin{array}{lllll}1 & -5.23407632 & 2.68010748 & 0.53924854\end{array}$

$\begin{array}{lllll}1 & -3.83278619 & 1.99392032 & -0.27814688\end{array}$

$1-5.408459261 .95482888-1.05711012$

$\begin{array}{llllll}6 & -5.20526414 & 0.50751594 & 0.54037504\end{array}$

$\begin{array}{lllll}1 & -6.28205271 & 0.44786053 & 0.71023616\end{array}$

$\begin{array}{lllll}1 & -4.74275288 & 0.45200899 & 1.53019862\end{array}$

$6-4.76052853-0.70241209-0.29409184$

$1-4.80977063-0.44315422-1.35645139$

$1-5.47338606-1.51689991-0.15029504$

$\begin{array}{lllll}6 & -3.36813507 & -1.24409560 & 0.02992631\end{array}$

$1-3.18720068-2.12838070-0.58822441$

$1-3.35694928-1.593508361 .06752426$

$6-2.21710005-0.26490486-0.17070137$

$\begin{array}{lllll}1 & -2.32065665 & 0.57529905 & 0.52124581\end{array}$

$\begin{array}{lllll}1 & -2.26993867 & 0.15921611 & -1.17876856\end{array}$

$\begin{array}{lllll}6 & -0.85034313 & -0.90543949 & 0.03675396\end{array}$ 


\begin{tabular}{lrrrr}
1 & -0.73036704 & -1.73709995 & -0.66485292 \\
1 & -0.80583129 & -1.34497731 & 1.03847239 \\
6 & 0.30640113 & 0.07052376 & -0.13637257 \\
1 & 0.18852800 & 0.89918970 & 0.56919720 \\
1 & 0.25798401 & 0.51427261 & -1.13596142 \\
6 & 1.67417892 & -0.56956648 & 0.06389342 \\
1 & 1.79292527 & -1.39698889 & -0.64294348 \\
1 & 1.72217144 & -1.01483116 & 1.06279766 \\
6 & 2.83012105 & 0.40784875 & -0.10717826 \\
1 & 2.71189787 & 1.23448081 & 0.60073583 \\
1 & 2.78091697 & 0.85428544 & -1.10553259 \\
6 & 4.19834970 & -0.23183074 & 0.09066825 \\
1 & 4.31759476 & -1.05798816 & -0.61781705 \\
1 & 4.24809757 & -0.67921046 & 1.08870816 \\
6 & 5.35492400 & 0.74591818 & -0.07927304 \\
1 & 5.23503502 & 1.56987030 & 0.62963072 \\
1 & 5.30346354 & 1.19247807 & -1.07614566 \\
6 & 6.71775787 & 0.09548158 & 0.11931913 \\
1 & 6.87357581 & -0.71178850 & -0.59866687 \\
1 & 6.80432563 & -0.33242849 & 1.11975278 \\
1 & 7.52713371 & 0.81478240 & -0.00686488 \\
\multicolumn{4}{c}{ energy } & \multicolumn{3}{c}{2.34247} \\
\multicolumn{4}{c}{ Symmetry point group: } & C1 \\
\multicolumn{4}{c}{ moment of inertia } & 2.1702
\end{tabular}

Cartesian coordinate of structure 80 $\begin{array}{llll}6 & -3.16027820 & 2.29024012 & 0.40522012\end{array}$ $\begin{array}{lllll}1 & -3.36840833 & 3.24414747 & 0.89021674\end{array}$

$\begin{array}{lllll}1 & -2.52835445 & 1.71079833 & 1.07932138\end{array}$

$1-2.583448492 .49286785-0.49929100$

$\begin{array}{lllll}6 & -4.45219881 & 1.55094748 & 0.07666790\end{array}$

$\begin{array}{llll}1 & -5.10001169 & 2.20711924-0.50923487\end{array}$

$\begin{array}{lllll}1 & -4.98958138 & 1.34270010 & 1.00631616\end{array}$

$\begin{array}{lllll}6 & -4.27092986 & 0.23884968 & -0.68843129\end{array}$

$1-3.821472290 .44720966-1.66445484$

$1-5.26342781-0.16686260-0.89382714$

$\begin{array}{lllll}6 & -3.43072483 & -0.82174250 & 0.03627080\end{array}$

$1-3.83669414-1.81478219-0.17155382$

$1-3.52787242-0.681720251 .11812101$

$6-1.95158150-0.82381360-0.34115664$

$\begin{array}{lllll}1 & -1.53747487 & 0.17776821 & -0.21679072\end{array}$

$1-1.85484554-1.06006049-1.40609939$

$\begin{array}{lllll}6 & -1.13682031 & -1.82145137 & 0.47362099\end{array}$

$\begin{array}{lllll}1 & -1.60232692 & -2.80780589 & 0.39117467\end{array}$

$1-1.19330235-1.547089341 .53223269$

$\begin{array}{llll}6 & 0.32871713-1.92890833 & 0.06055638\end{array}$

$10.38891685-2.27282275-0.97715153$

$1 \quad 0.80869941-2.704022830 .66459863$

$\begin{array}{llll}6 & 1.12297946 & -0.63419761 & 0.20209110\end{array}$ 
$1 \quad 0.97003736-0.22198757 \quad 1.20525115$

$1 \quad 0.74136167 \quad 0.11525827-0.49640605$

$62.61454905-0.82431918-0.04256750$

$12.76331126-1.27440244-1.02955397$

$1 \quad 3.00860584-1.545306660 .68089319$

$\begin{array}{lllll}6 & 3.41603454 & 0.46788631 & 0.04615722\end{array}$

$\begin{array}{llll}1 & 3.25615468 & 0.92774249 & 1.02692116\end{array}$

$13.034097321 .18335358-0.68931024$

$\begin{array}{llll}6 & 4.91039463 & 0.26884889-0.17632241\end{array}$

$15.06822918-0.19471031-1.15410404$

$15.29049832-0.442611080 .56201676$

$65.70356831 \quad 1.56633868-0.09221265$

$1 \quad 5.584788902 .034024490 .88697784$

$15.362138432 .28261914-0.84175024$

$1 \quad 6.768123781 .39650411-0.25387768$ energy $\quad 2.44295$

Symmetry point group: C1 moment of inertia 2.0978

Cartesian coordinate of structure 81 $6-3.49380898-2.34930826-0.54844743$

$1-3.73109971-2.95288793-1.42523711$

$1-2.47069664-1.99144435-0.65766901$

$1-3.51962903-3.005690560 .32328460$

$6-4.48746374-1.20254127-0.38400257$

$1-5.48887487-1.62971083-0.30259403$

$1-4.49655003-0.58887796-1.28959496$

$\begin{array}{lllll}6 & -4.22507731 & -0.30378469 & 0.83294588\end{array}$

$1-3.77307838-0.902401491 .63021845$

$\begin{array}{lllll}1 & -5.17970454 & 0.05030080 & 1.22738566\end{array}$

$\begin{array}{lllll}6 & -3.35228401 & 0.92219339 & 0.56454148\end{array}$

$\begin{array}{lllll}1 & -3.27877490 & 1.50462257 & 1.48777348\end{array}$

$\begin{array}{llll}1 & -3.86037633 & 1.56733402 & -0.15965687\end{array}$

$\begin{array}{lllll}6 & -1.94368421 & 0.63010914 & 0.05969200\end{array}$

$\begin{array}{lllll}1 & -1.99278425 & 0.15858932 & -0.92609636\end{array}$

$\begin{array}{lllll}1 & -1.46847190 & -0.09543048 & 0.72551488\end{array}$

$\begin{array}{lllll}6 & -1.08590568 & 1.88715127 & -0.04130545\end{array}$

$\begin{array}{lllll}1 & -0.96171990 & 2.31842503 & 0.95731718\end{array}$

$1-1.626533442 .63399209-0.62965262$

$\begin{array}{lllll}6 & 0.28782846 & 1.66322580 & -0.66862779\end{array}$

$1 \quad 0.785580392 .62981135-0.78639291$

$1 \quad 0.161369551 .26110921-1.67913527$

$\begin{array}{lllll}6 & 1.20041253 & 0.73639631 & 0.12735369\end{array}$

$\begin{array}{llll}1 & 0.75428754 & -0.25948848 & 0.19405589\end{array}$

$\begin{array}{llll}1 & 1.27932891 & 1.10491082 & 1.15562151\end{array}$

$\begin{array}{lllll}6 & 2.59537714 & 0.61237831 & -0.47251093\end{array}$

$13.053918751 .60472890-0.53097570$

$1 \quad 2.513855240 .25488770-1.50408293$

$\begin{array}{lllll}6 & 3.51030654 & -0.31943784 & 0.31162871\end{array}$ 
$1 \quad 3.05257243-1.312350330 .36917398$

$\begin{array}{lllll}1 & 3.59123237 & 0.03692227 & 1.34372032\end{array}$

$64.90612510-0.44441863-0.28714697$

$1 \quad 5.362074240 .54786957-0.34448441$

$14.82367877-0.80119598-1.31755219$

$6 \quad 5.81180794-1.37771103 \quad 0.50564425$

$1 \quad 5.39144601-2.384018770 .55155871$

$15.93421772-1.024986441 .53135030$

$1 \quad 6.80292945-1.45053503 \quad 0.05756031$ energy $\quad 2.68633$

Symmetry point group: C1 moment of inertia 2.1768

Cartesian coordinate of structure 82

$65.30889290-0.958343450 .66362765$

$1 \quad 6.11677302-1.301075261 .31057314$

$14.37956443-1.376654561 .05272789$

$15.47315090-1.38096858-0.32947440$

$\begin{array}{lllll}6 & 5.26032336 & 0.56415233 & 0.61490337\end{array}$

$\begin{array}{lllll}1 & 6.24092501 & 0.94408557 & 0.31853369\end{array}$

$\begin{array}{lllll}1 & 5.07884681 & 0.94766618 & 1.62303761\end{array}$

$\begin{array}{lllll}6 & 4.20639351 & 1.14959200 & -0.32610917\end{array}$

$14.437267780 .86070018-1.35636564$

$14.29820300 \quad 2.23688648-0.29148591$

$6 \quad 2.761026990 .74575594-0.00169445$

$12.099174631 .59555852-0.17690502$

$\begin{array}{lllll}1 & 2.67699648 & 0.51765368 & 1.06665022\end{array}$

$6 \quad 2.25471279-0.44566717-0.81464632$

$12.99726682-1.24520778-0.79105919$

$12.17033148-0.14354378-1.86354077$

$6 \quad 0.91512188-1.00370293-0.34283451$

$1 \quad 1.02200501-1.381796040 .67931862$

$1 \quad 0.65746658-1.86928318-0.96001314$

$6-0.23884484-0.00759158-0.38915169$

$\begin{array}{llll}1 & -0.27135815 & 0.46312723 & -1.37754591\end{array}$

$\begin{array}{lllll}1 & -0.06342657 & 0.79922486 & 0.32754813\end{array}$

$\begin{array}{llll}6 & -1.58888892 & -0.64931470 & -0.09449474\end{array}$

$\begin{array}{lllll}1 & -1.54695572 & -1.14822734 & 0.87906745\end{array}$

$1-1.78051157-1.43643320-0.83079291$

$\begin{array}{lllll}6 & -2.74731049 & 0.33969162 & -0.10435285\end{array}$

$1-2.772165530 .85659323-1.06911174$

$\begin{array}{lllll}1 & -2.57013743 & 1.11296873 & 0.65000250\end{array}$

$\begin{array}{llll}6 & -4.10081821 & -0.31147512 & 0.14961419\end{array}$

$\begin{array}{lllll}1 & -4.07799668 & -0.83082597 & 1.11315798\end{array}$

$1-4.27768502-1.08311464-0.60672614$

$\begin{array}{lllll}6 & -5.26053019 & 0.67692999 & 0.13859844\end{array}$

$\begin{array}{lllll}1 & -5.27538317 & 1.20045102 & -0.82141019\end{array}$

$\begin{array}{lllll}1 & -5.08703666 & 1.44265687 & 0.89986378\end{array}$

$\begin{array}{lllll}6 & -6.61143254 & 0.01571559 & 0.37843185\end{array}$ 


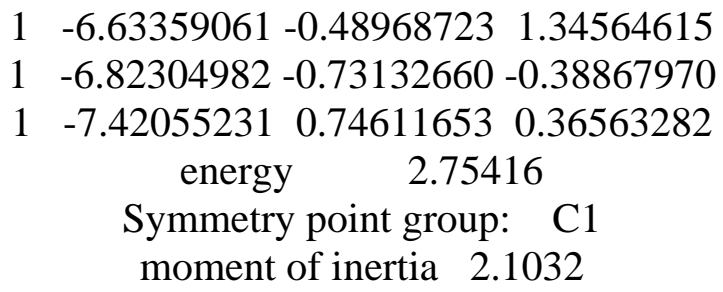

Cartesian coordinate of structure 83

$\begin{array}{llll}6 & 5.24488446 & -0.05892037 & 0.37945728\end{array}$

$\begin{array}{lllll}1 & 6.31734152 & 0.01566314 & 0.19805950\end{array}$

$\begin{array}{lllll}1 & 4.78995034 & 0.87176684 & 0.03798254\end{array}$

$1 \quad 5.09187127-0.127582681 .45825044$

$64.65457418-1.26763809-0.33728558$

$15.21293982-2.16117972-0.04837470$

$14.80345010-1.15150023-1.41465892$

$63.16881691-1.51568134-0.07277599$

$13.02147748-1.730113340 .99056433$

$12.88325670-2.42489175-0.60530764$

$6 \quad 2.23875009-0.36703567-0.48838963$

$1 \quad 1.31472472-0.78271066-0.89541985$

$1 \quad 2.698318740 .19594551-1.30818964$

$\begin{array}{lllll}6 & 1.88467419 & 0.58995465 & 0.64836997\end{array}$

$\begin{array}{llll}1 & 2.79581256 & 0.91030778 & 1.15636463\end{array}$

$\begin{array}{lllll}1 & 1.30492663 & 0.04386175 & 1.39792027\end{array}$

$\begin{array}{lllll}6 & 1.11728874 & 1.83361958 & 0.20101114\end{array}$

$1 \quad 1.765851492 .43329348-0.44416208$

$\begin{array}{lllll}1 & 0.90584939 & 2.44970140 & 1.07951376\end{array}$

$\begin{array}{llll}6 & -0.19329629 & 1.56323109-0.53810968\end{array}$

$10.011596991 .05977520-1.48713079$

$1-0.647811582 .52226820-0.80133207$

$\begin{array}{lllll}6 & -1.20135341 & 0.74068258 & 0.25584161\end{array}$

$\begin{array}{lllll}1 & -1.36612882 & 1.21181079 & 1.23058232\end{array}$

$\begin{array}{llll}1 & -0.78959975 & -0.25109240 & 0.46424094\end{array}$

$\begin{array}{lllll}6 & -2.53637878 & 0.57912330 & -0.45994707\end{array}$

$1-2.366816580 .12103845-1.43979744$

$1-2.963300921 .56760845-0.65734562$

$\begin{array}{lllll}6 & -3.54303868 & -0.25776184 & 0.31880758\end{array}$

$\begin{array}{lllll}1 & -3.70973351 & 0.19747402 & 1.30049295\end{array}$

$\begin{array}{lllll}1 & -3.11766897 & -1.24785611 & 0.51250632\end{array}$

$\begin{array}{llll}6 & -4.88043027-0.41694500 & -0.39426282\end{array}$

$1-4.71237439-0.87101146-1.37477819$

$1 \quad-5.304666620 .57252093-0.58600964$

$\begin{array}{llll}6 & -5.87789550 & -1.25643663 & 0.39324908\end{array}$

$\begin{array}{lllll}1 & -6.08476077 & -0.80659257 & 1.36603710\end{array}$

$1-5.48871442-2.260631150 .57101310$

$1-6.82536519-1.35502739-0.13681700$ energy $\quad 2.77732$

Symmetry point group: C1 moment of inertia 2.1039 
Cartesian coordinate of structure 84

$6-5.23918518-0.25942307-0.13778654$

$\begin{array}{llllll}1 & -6.29426998 & -0.38567397 & 0.10623368\end{array}$

$\begin{array}{lllll}1 & -4.86476879 & 0.57610427 & 0.45479113\end{array}$

$1 \quad-5.170015760 .02193151-1.19039889$

$\begin{array}{llll}6 & -4.45853186 & -1.53845159 & 0.14105906\end{array}$

$1-4.94185607-2.37083750-0.37589953$

$\begin{array}{lllll}1 & -4.51823779 & -1.76815696 & 1.20877288\end{array}$

$6-2.98628378-1.49977215-0.27110006$

$1-2.91944072-1.36557447-1.35544554$

$1-2.55667802-2.48174666-0.06363508$

$\begin{array}{lllll}6 & -2.14411904 & -0.42015251 & 0.42445959\end{array}$

$1-1.15245709-0.818029120 .64305064$

$1-2.58834171-0.183750061 .39696658$

$\begin{array}{lllll}6 & -1.99159314 & 0.87310841 & -0.37786477\end{array}$

$\begin{array}{lllll}1 & -2.98336567 & 1.27288876 & -0.59279459\end{array}$

$\begin{array}{lllll}1 & -1.55072300 & 0.63647366 & -1.35181113\end{array}$

$\begin{array}{lllll}6 & -1.14464122 & 1.95051207 & 0.31509574\end{array}$

$\begin{array}{llllll}1 & -1.21478774 & 1.82135997 & 1.39974950\end{array}$

$\begin{array}{lllll}1 & -1.56842403 & 2.93390613 & 0.10275232\end{array}$

$\begin{array}{lllll}6 & 0.32801899 & 1.97991428 & -0.09441779\end{array}$

$\begin{array}{lllll}1 & 0.81753883 & 2.81168961 & 0.42055809\end{array}$

$1 \quad 0.393412882 .20545560-1.16382540$

$\begin{array}{lllll}6 & 1.11046378 & 0.70237927 & 0.18672794\end{array}$

$10.69104454-0.12405482-0.39379693$

$\begin{array}{lllll}1 & 0.99330926 & 0.42743609 & 1.24009184\end{array}$

$6 \quad 2.593050490 .82914511-0.14052131$

$\begin{array}{lllll}1 & 3.02513217 & 1.64504553 & 0.44766466\end{array}$

$12.707323351 .11851216-1.19020743$

$63.37990190-0.449346050 .11745044$

$12.94922695-1.26468890-0.47288189$

$1 \quad 3.26327741-0.740656011 .16639610$

$64.86372882-0.32460915-0.20678931$

$1 \quad 5.292772520 .489513940 .38390255$

$14.97859982-0.03341318-1.25455759$

$6 \quad 5.63951539-1.609021720 .05442947$

$15.24831267-2.43098820-0.54789468$

$15.56461360-1.904280841 .10260405$

$16.69685140-1.49416401-0.18484003$ energy $\quad 4.57137$

Symmetry point group: C1 moment of inertia 2.2512

Cartesian coordinate of structure 85

$\begin{array}{llll}6 & -5.32020411 & -0.34066904 & 1.27807992\end{array}$

$1-5.67415326-0.987574272 .08158583$

$\begin{array}{lllll}1 & -4.56813831 & 0.32752389 & 1.69572295\end{array}$

$\begin{array}{lllll}1 & -6.16091909 & 0.27490737 & 0.95297351\end{array}$ 


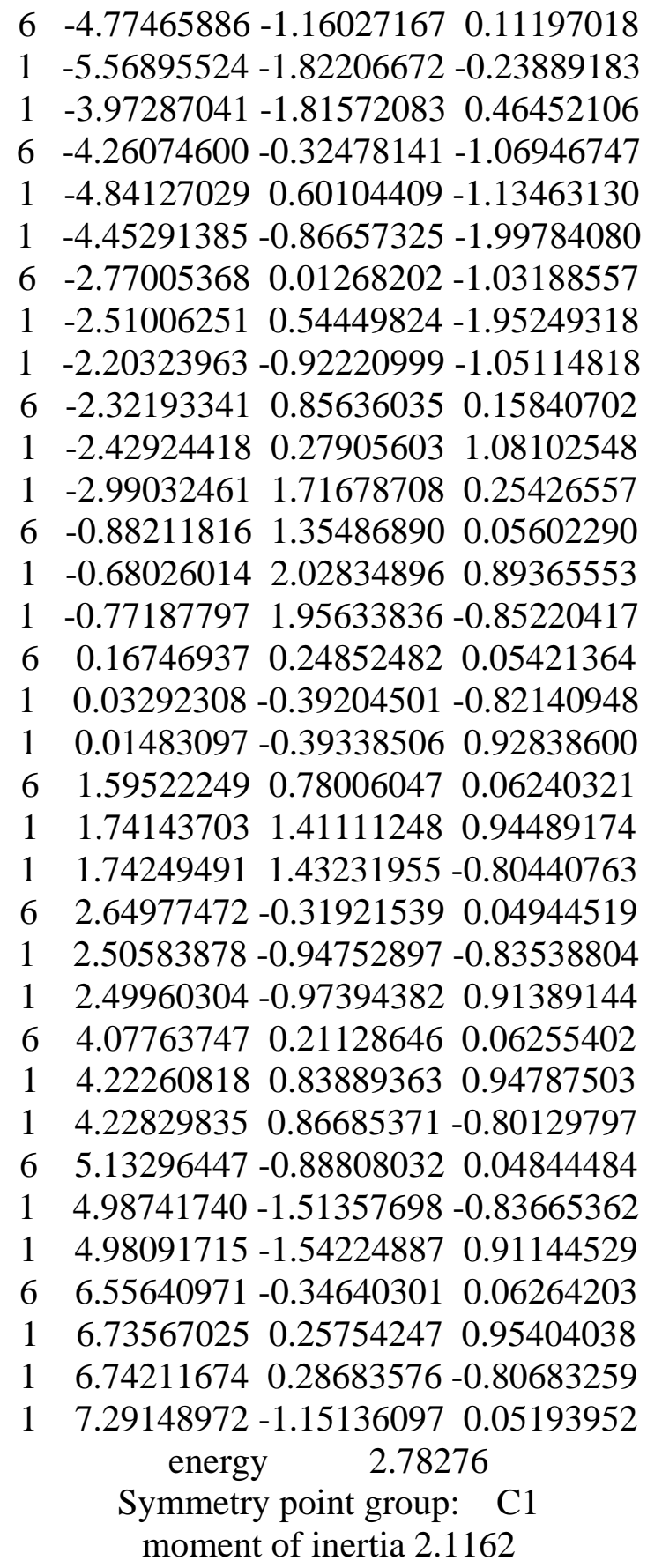

Cartesian coordinate of structure 86

$\begin{array}{llll}6 & -5.05405070 & -0.67308138 & 0.81197098\end{array}$

$\begin{array}{lllll}1 & -5.50570894 & -0.94647182 & 1.76625160\end{array}$

$\begin{array}{lllll}1 & -4.75434418 & 0.37243213 & 0.87282906\end{array}$

$\begin{array}{lllll}1 & -5.82724848 & -0.74801003 & 0.04520355\end{array}$

$\begin{array}{lllll}6 & -3.87858652 & -1.58704495 & 0.47725539\end{array}$

$1-4.24342201-2.615776960 .45535745$

$1-3.14001090-1.548713661 .28342676$

$6-3.18653889-1.27170592-0.85694519$

$1-3.92113323-0.85632565-1.55414720$

$1-2.83854462-2.20393957-1.30648176$ 
$6-1.98685013-0.32839148-0.76341373$

$1-1.53746760-0.23562293-1.75668238$

$1-1.22890161-0.79618883-0.12832622$

$\begin{array}{lllll}6 & -2.29977723 & 1.06738431 & -0.23366329\end{array}$

$\begin{array}{lllll}1 & -2.61524744 & 0.99712145 & 0.81115716\end{array}$

$1-3.154314331 .47104750-0.78490904$

$\begin{array}{lllll}6 & -1.14395638 & 2.06471226 & -0.32097291\end{array}$

$\begin{array}{lllll}1 & -1.51296492 & 3.03141717 & 0.02703062\end{array}$

$\begin{array}{lllll}1 & -0.86688761 & 2.20756691 & -1.37025049\end{array}$

$\begin{array}{lllll}6 & 0.10562544 & 1.68219027 & 0.48449360\end{array}$

$\begin{array}{llll}1 & -0.19150363 & 1.07868743 & 1.34872419\end{array}$

$\begin{array}{lllll}1 & 0.56334966 & 2.58528680 & 0.89595573\end{array}$

$\begin{array}{lllll}6 & 1.17266641 & 0.93508412 & -0.31129036\end{array}$

$1 \quad 1.510398841 .57243517-1.13467068$

$1 \quad 0.742169290 .04568496-0.77686246$

$\begin{array}{lllll}6 & 2.37208347 & 0.52650244 & 0.53376423\end{array}$

$1 \quad 2.03322127-0.113525081 .35492218$

$\begin{array}{lllll}1 & 2.80582656 & 1.41595487 & 1.00225986\end{array}$

$63.44981454-0.20204241-0.25879725$

$1 \quad 3.789857380 .43719021-1.08003237$

$13.01528370-1.09114489-0.72721967$

$\begin{array}{lllll}6 & 4.64933234 & -0.61519544 & 0.58548498\end{array}$

$14.30797076-1.25241387 \quad 1.40601598$

$1 \quad 5.083479920 .27383648 \quad 1.05125370$

$65.71836696-1.34508749-0.21718038$

$16.09668123-0.71689409-1.02571351$

$15.31583274-2.25428485-0.66752093$

$1 \quad 6.56485230-1.629294870 .40819245$ energy $\quad 4.45372$

Symmetry point group: C1 moment of inertia 2.0507

Cartesian coordinate of structure 87

$\begin{array}{llll}6 & 2.06714503 & 2.35831808 & 0.75352562\end{array}$

$\begin{array}{lllll}1 & 1.69437755 & 3.38190351 & 0.79798608\end{array}$

$\begin{array}{lllll}1 & 1.20203912 & 1.70060643 & 0.66401037\end{array}$

$1 \quad 2.554782142 .137124431 .70485816$

$\begin{array}{llll}6 & 3.03155634 & 2.17991294 & -0.41317148\end{array}$

$13.805168922 .94924169-0.35501079$

$1 \quad 2.496387432 .35811972-1.35033607$

$\begin{array}{llll}6 & 3.71431564 & 0.81364910 & -0.48950975\end{array}$

$\begin{array}{lllll}1 & 4.32636837 & 0.66562594 & 0.40588968\end{array}$

$14.411492350 .83870470-1.32940393$

$6 \quad 2.76675793-0.38223616-0.65861376$

$13.24220180-1.13548286-1.29278825$

$1 \quad 1.87536023-0.05468516-1.20217605$

$6 \quad 2.36127541-1.05864561 \quad 0.64919402$

$1 \quad 1.84852005-0.345460861 .29702352$

$1 \quad 3.26904957-1.347377721 .18584095$ 


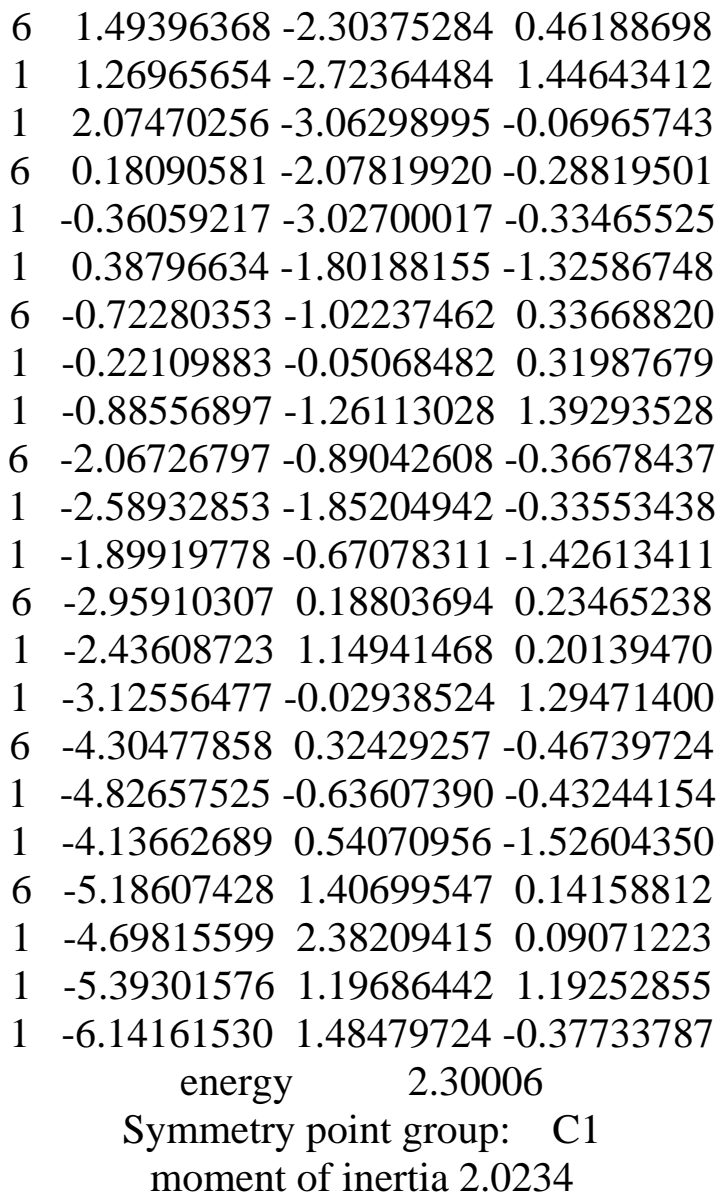

Cartesian coordinate of structure 88

$63.294533932 .11601366-0.33714832$

$13.565350842 .95721154-0.97612025$

$12.461748961 .59744735-0.81071023$

$\begin{array}{lllll}1 & 2.93242503 & 2.52184378 & 0.60941320\end{array}$

$\begin{array}{lllll}6 & 4.49273558 & 1.19891480 & -0.10463187\end{array}$

$1 \quad 5.30620076 \quad 1.80467966 \quad 0.29974794$

$14.853594450 .81479216-1.06331507$

$\begin{array}{lllll}6 & 4.23416958 & 0.02224610 & 0.84626071\end{array}$

$1 \quad 3.53114296 \quad 0.334398401 .62355069$

$15.16273943-0.217930011 .36827977$

$6 \quad 3.74588491-1.267604730 .18095881$

$13.65407602-2.039432040 .95089162$

$14.52436583-1.61418958-0.50461016$

$6 \quad 2.42727160-1.18321447-0.58532307$

$12.23608524-2.15281232-1.05359666$

$12.52346682-0.47187568-1.41034251$

$\begin{array}{lllll}6 & 1.22408423 & -0.80895724 & 0.27165047\end{array}$

$\begin{array}{lllll}1 & 1.40038009 & 0.15006544 & 0.76576677\end{array}$

$1 \quad 1.10983198-1.546254141 .07292997$

$6-0.07051873-0.71939613-0.52575121$

$1-0.25633431-1.67471365-1.02696673$

$1 \quad 0.049875410 .02217868-1.32256499$ 


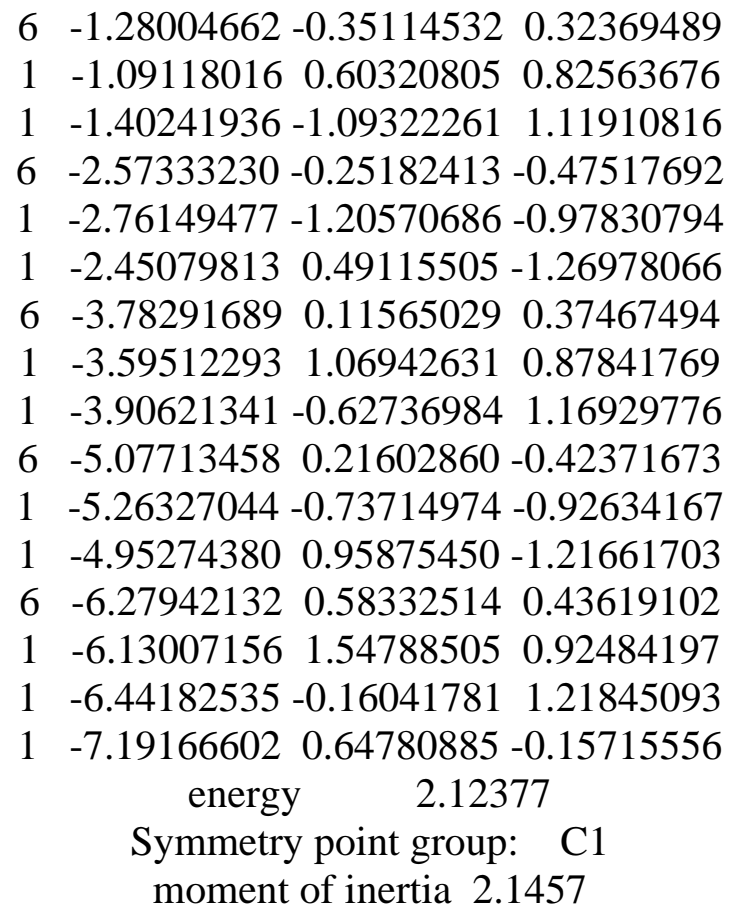

Cartesian coordinate of structure $\quad 89$

$\begin{array}{llll}6 & -2.24987366 & 2.16478221 & -0.52492769\end{array}$

$\begin{array}{lllll}1 & -1.96608089 & 3.18953750 & -0.28275774\end{array}$

$\begin{array}{lllll}1 & -1.42714111 & 1.51649813 & -0.22703874\end{array}$

$1-2.345625342 .09525756-1.61028035$

$\begin{array}{lllll}6 & -3.56335053 & 1.78701904 & 0.15519146\end{array}$

$\begin{array}{lllll}1 & -4.31470114 & 2.52626887 & -0.12984846\end{array}$

$\begin{array}{lllll}1 & -3.45411969 & 1.87448353 & 1.24024922\end{array}$

$\begin{array}{lllll}6 & -4.09433929 & 0.38838812 & -0.18773821\end{array}$

$\begin{array}{llll}1 & -3.84665657 & 0.15287988 & -1.22651976\end{array}$

$1-5.185228900 .40704621-0.14178438$

$\begin{array}{lllll}6 & -3.62540680 & -0.74017369 & 0.73477166\end{array}$

$\begin{array}{lllll}1 & -4.12527660 & -1.66451096 & 0.43009045\end{array}$

$1-3.97931122-0.523024441 .74661810$

$\begin{array}{llll}6 & -2.12030728 & -0.99535470 & 0.79083034\end{array}$

$1-1.92906842-1.778610751 .53019960$

$\begin{array}{llll}1 & -1.61433214 & -0.10373383 & 1.16672002\end{array}$

$6-1.51389766-1.42816105-0.54048632$

$1-1.67189604-0.65079498-1.29300253$

$1-2.05443971-2.30984501-0.89658132$

$6-0.02241147-1.74853625-0.47059956$

$1 \quad 0.28802999-2.20524773-1.41440307$

$1 \quad 0.15165508-2.502131130 .30436996$

$6 \quad 0.86440638-0.53689750-0.20262483$

$1 \quad 0.60224703-0.085406980 .75818427$

$1 \quad 0.666644490 .22651691-0.96240472$

$62.35074464-0.87076226-0.20048632$

$12.62308195-1.31535746-1.16309979$

$1 \quad 2.54709660-1.638620030 .55469714$ 


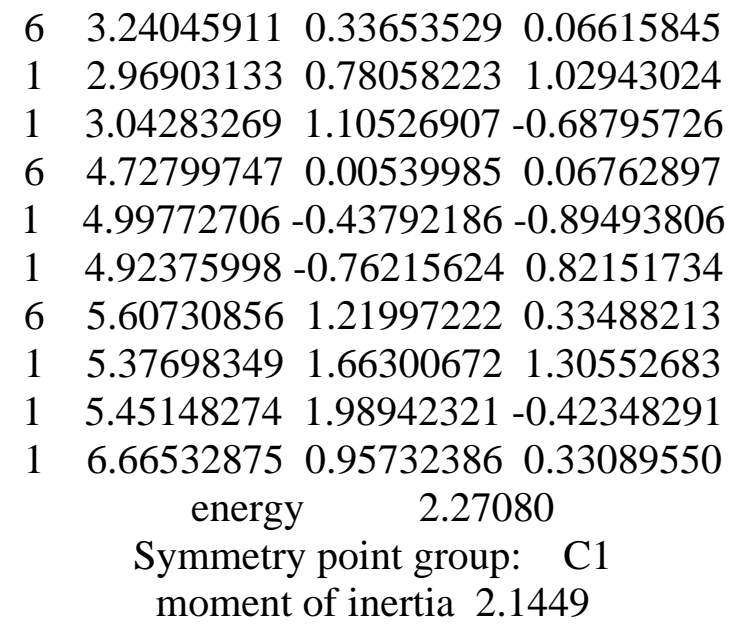

Cartesian coordinate of structure 90 $\begin{array}{lllll}6 & -2.62725073 & 2.09781634 & 0.81883177\end{array}$ $\begin{array}{lllll}1 & -2.92853944 & 2.98566932 & 1.37603977\end{array}$ $\begin{array}{lllll}1 & -2.40622801 & 1.31533279 & 1.54248858\end{array}$ $\begin{array}{lllll}1 & -1.69641037 & 2.33185158 & 0.29941209\end{array}$ $\begin{array}{llll}6 & -3.71408068 & 1.68786516-0.17044388\end{array}$ $1-3.948013852 .55779150-0.78836539$ $\begin{array}{lllll}1 & -4.63449194 & 1.45223185 & 0.37199428\end{array}$ $\begin{array}{llll}6 & -3.37055755 & 0.51783582 & -1.10056504\end{array}$ $\begin{array}{llll}1 & -2.33822423 & 0.61321768-1.44452395\end{array}$ $1 \quad-3.985951760 .61422005-1.99823657$ $6-3.63099458-0.88574921-0.54516405$ $1-3.55585751-1.59778264-1.37278361$

$1-4.67549058-0.91981625-0.22015968$

$\begin{array}{lllll}6 & -2.76287781 & -1.41805983 & 0.59873363\end{array}$

$\begin{array}{llll}1 & -3.23762479-2.33888838 & 0.94554833\end{array}$

$\begin{array}{llll}1 & -2.79098609 & -0.73725115 & 1.45216243\end{array}$

$\begin{array}{lllll}6 & -1.30737875 & -1.74648043 & 0.24882009\end{array}$

$1-1.27066460-2.20339962-0.74563028$

$\begin{array}{llll}1 & -0.95217721 & -2.51427427 & 0.94197706\end{array}$

$\begin{array}{lllll}6 & -0.32119935 & -0.58386104 & 0.30814734\end{array}$

$\begin{array}{llll}1 & -0.38407474 & -0.11077836 & 1.29295012\end{array}$

$\begin{array}{lllll}1 & -0.59462321 & 0.18803399 & -0.41294735\end{array}$

$\begin{array}{llll}6 & 1.11631190 & -1.01637663 & 0.04996009\end{array}$

$11.18262942-1.48943390-0.93504362$

$1 \quad 1.39776960-1.786524780 .77535510$

$\begin{array}{lllll}6 & 2.11473307 & 0.13185587 & 0.12575976\end{array}$

$\begin{array}{lllll}1 & 2.03806918 & 0.61135549 & 1.10683183\end{array}$

$1 \quad 1.841961750 .89711226-0.60793755$

$63.55493701-0.30353379-0.11112054$

$13.63383297-0.77993223-1.09366675$

$13.82462348-1.072189560 .62036264$

$\begin{array}{lllll}6 & 4.55795147 & 0.84086097 & -0.02628802\end{array}$

$14.474526581 .31841090 \quad 0.95381604$

$14.292032001 .60558195-0.76118678$ 


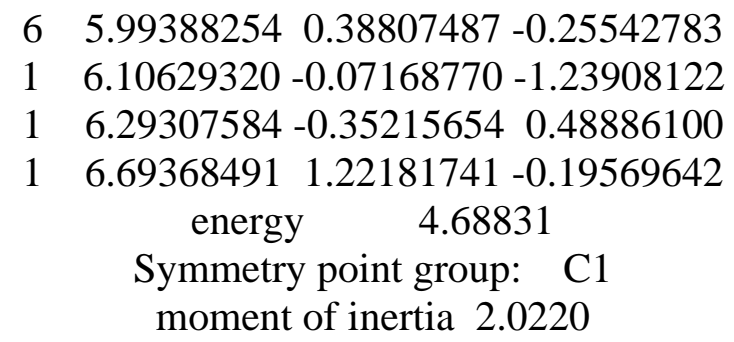

Cartesian coordinate of structure 91

6 -4.11199291 -1.74816663 -0.66303602

$1-4.98202846-2.27233923-1.05990132$

$1-3.70705390-1.13115360-1.46372933$

$1-3.35896652-2.49736180-0.41148926$

$\begin{array}{lllll}6 & -4.48642391 & -0.92799365 & 0.56846491\end{array}$

$\begin{array}{lllll}1 & -4.96343940 & -1.59870186 & 1.28617927\end{array}$

$1-5.24524395-0.187501560 .29958470$

$\begin{array}{llll}6 & -3.31720681 & -0.21871234 & 1.26424258\end{array}$

$1-2.42702223-0.853380961 .21518263$

$\begin{array}{llll}1 & -3.55160732 & -0.12185918 & 2.32643766\end{array}$

$\begin{array}{lllll}6 & -2.99044254 & 1.18535023 & 0.74976816\end{array}$

$\begin{array}{lllll}1 & -2.23808897 & 1.62467149 & 1.40952159\end{array}$

$\begin{array}{lllll}1 & -3.88268019 & 1.80794142 & 0.86074526\end{array}$

$\begin{array}{lllll}6 & -2.50819279 & 1.29314681 & -0.69811861\end{array}$

$1-2.305324462 .34696193-0.90976555$

$1-3.316178251 .00564954-1.37468497$

$\begin{array}{llll}6 & -1.26085539 & 0.47762960 & -1.03499887\end{array}$

$\begin{array}{llll}1 & -1.03525449 & 0.61086721 & -2.09663426\end{array}$

$1-1.46383295-0.58841625-0.90335469$

$\begin{array}{lllll}6 & -0.02909518 & 0.85102209 & -0.21824190\end{array}$

$\begin{array}{lllll}1 & -0.22300724 & 0.68370739 & 0.84495432\end{array}$

$1 \quad 0.166241591 .92284361-0.32815130$

$\begin{array}{llll}6 & 1.21221280 & 0.06505608 & -0.62131262\end{array}$

$1 \quad 1.40861395 \quad 0.22013513-1.68695511$

$1 \quad 1.01545945-1.00511524-0.50105015$

$\begin{array}{lllll}6 & 2.45307808 & 0.44055494 & 0.17885543\end{array}$

$\begin{array}{lllll}1 & 2.25497263 & 0.29275399 & 1.24525668\end{array}$

$\begin{array}{lllll}1 & 2.65385235 & 1.50909512 & 0.05169171\end{array}$

$63.69028889-0.35527271-0.21715576$

$13.88419938-0.21373327-1.28530117$

$13.49191608-1.42358329-0.08332178$

$\begin{array}{lllll}6 & 4.93614236 & 0.02593319 & 0.57373671\end{array}$

$14.74155967-0.114226371 .64057758$

$\begin{array}{lllll}1 & 5.13435631 & 1.09253559 & 0.43696604\end{array}$

$\begin{array}{lllll}6 & 6.16436441 & -0.77898701 & 0.16871917\end{array}$

$1 \quad 6.39194475-0.63495217-0.88906967$

$1 \quad 6.00143755-1.84633807 \quad 0.32850615$

$17.04391258-0.485863160 .74226590$ energy $\quad 2.17530$

Symmetry point group: C1 
moment of inertia 2.0244

Cartesian coordinate of structure 92

$\begin{array}{lllll}6 & -6.07555510 & 1.24963890 & 0.45355988\end{array}$

$\begin{array}{lllll}1 & -7.06775042 & 1.64439443 & 0.23461307\end{array}$

$\begin{array}{lllll}1 & -5.99239185 & 1.13601199 & 1.53645561\end{array}$

$\begin{array}{lllll}1 & -5.34854165 & 2.00027328 & 0.14318847\end{array}$

$6-5.84405176-0.08382995-0.24894869$

$\begin{array}{lllll}1 & -5.88559714 & 0.06329600 & -1.33195465\end{array}$

$1-6.66188624-0.76429787-0.00187756$

$\begin{array}{llll}6 & -4.52206151 & -0.75615513 & 0.11775349\end{array}$

$1-4.47977626-1.74079583-0.35597217$

$\begin{array}{lllll}1 & -4.49929750 & -0.93561676 & 1.19781869\end{array}$

$\begin{array}{lllll}6 & -3.27985472 & 0.03357855 & -0.28034846\end{array}$

$\begin{array}{lllll}1 & -3.28328646 & 1.00768058 & 0.21561051\end{array}$

$1-3.311065750 .23718202-1.35591116$

$\begin{array}{llll}6 & -1.98102072 & -0.68550121 & 0.06270250\end{array}$

$1-1.95711092-1.65696953-0.44086062$

$\begin{array}{llll}1 & -1.96306751 & -0.89910238 & 1.13637175\end{array}$

$\begin{array}{lllll}6 & -0.73514955 & 0.10867655 & -0.30903856\end{array}$

$\begin{array}{lllll}1 & -0.77874005 & 1.09057918 & 0.17290657\end{array}$

$\begin{array}{lllll}1 & -0.73396801 & 0.29904324 & -1.38701279\end{array}$

$\begin{array}{lllll}6 & 0.56401663 & -0.58286382 & 0.08454339\end{array}$

$1 \quad 0.61719519-1.56307208-0.39956489$

$1 \quad 0.55486102-0.775956001 .16211940$

$\begin{array}{llll}6 & 1.80724879 & 0.22397548 & -0.26838913\end{array}$

$\begin{array}{lllll}1 & 1.73303193 & 1.21494184 & 0.19095401\end{array}$

$1 \quad 1.83730637 \quad 0.39189638-1.34961997$

$\begin{array}{lllll}6 & 3.10574651 & -0.43654030 & 0.17714077\end{array}$

$13.18843070-1.42561091-0.28462818$

$13.06882161-0.607647541 .25779525$

$\begin{array}{llll}6 & 4.34561208 & 0.38219788 & -0.15915681\end{array}$

$\begin{array}{lllll}1 & 4.25014220 & 1.37794179 & 0.28617496\end{array}$

$14.397184340 .53763096-1.24159435$

$6 \quad 5.64353661-0.25683002 \quad 0.31992133$

$15.74275176-1.24940816-0.12822655$

$15.58642803-0.414611061 .40046286$

$\begin{array}{lllll}6 & 6.87422675 & 0.57747782 & -0.01072538\end{array}$

$\begin{array}{lllll}1 & 6.80844184 & 1.56595707 & 0.44765215\end{array}$

$1 \quad 6.974142750 .71973611-1.08824826$

$\begin{array}{lllll}1 & 7.78757801 & 0.10357467 & 0.34926194\end{array}$ energy $\quad 0.389432$

Symmetry point group: C1 moment of inertia 2.3493

Cartesian coordinate of structure 93 $\begin{array}{lllll}6 & -4.62589400 & 2.27991970 & 0.08264539\end{array}$

$\begin{array}{lllll}1 & -5.25581197 & 3.08439947 & -0.29735670\end{array}$

$\begin{array}{lllll}1 & -4.60936426 & 2.35523866 & 1.17187553\end{array}$ 
$1 \quad-3.612449682 .45990821-0.27686542$

$\begin{array}{lllll}6 & -5.14877457 & 0.91723858 & -0.35885776\end{array}$

$1-5.129360720 .85607064-1.45076472$

$1-6.197516400 .82725173-0.06723538$

$\begin{array}{lllll}6 & -4.37474975 & -0.26468868 & 0.22191678\end{array}$

$1-4.88480971-1.19209836-0.05336758$

$1-4.40595578-0.212787991 .31532076$

$6-2.92172655-0.35062167-0.23264165$

$\begin{array}{lllll}1 & -2.38665656 & 0.54802877 & 0.08045011\end{array}$

$1-2.88659102-0.36390395-1.32756447$

$\begin{array}{llll}6 & -2.20637675 & -1.58476689 & 0.30703790\end{array}$

$\begin{array}{llll}1 & -2.78414660 & -2.47254815 & 0.03510248\end{array}$

$\begin{array}{llll}1 & -2.20300843 & -1.54764552 & 1.40134969\end{array}$

$6-0.77424157-1.75419243-0.19475319$

$1-0.77878369-1.80347715-1.28852843$

$\begin{array}{llll}1 & -0.38993353 & -2.71785715 & 0.15103506\end{array}$

$\begin{array}{lllll}6 & 0.18569884 & -0.65696541 & 0.25276924\end{array}$

$1 \quad 0.14892832-0.569894161 .34381173$

$\begin{array}{llll}1 & -0.14093012 & 0.30906913 & -0.14121233\end{array}$

$6 \quad 1.62351630-0.90637703-0.18499759$

$11.65701236-1.00340255-1.27494227$

$1 \quad 1.96268373-1.86712990 \quad 0.21536414$

$\begin{array}{lllll}6 & 2.58728812 & 0.18944107 & 0.25206111\end{array}$

$\begin{array}{llll}1 & 2.55391113 & 0.28615328 & 1.34201971\end{array}$

$12.24744597 \quad 1.15010950-0.14803170$

$64.02475729-0.05765857-0.18720877$

$14.05873547-0.15308674-1.27737424$

$14.36530693-1.018736040 .21160143$

$\begin{array}{lllll}6 & 4.98944937 & 1.03771682 & 0.25101823\end{array}$

$\begin{array}{lllll}1 & 4.95564568 & 1.13073534 & 1.34006711\end{array}$

$14.647340671 .99713749-0.14685249$

$\begin{array}{llll}6 & 6.42289589 & 0.78153952 & -0.19552121\end{array}$

$1 \quad 6.487226730 .71536237-1.28315558$

$\begin{array}{lllll}1 & 6.79765229 & -0.15823371 & 0.21417748\end{array}$

$1 \quad 7.09237366 \quad 1.577826680 .13026526$ energy $\quad 0.905737$

Symmetry point group: C1 moment of inertia 2.3794

Cartesian coordinate of structure 94

$65.22811726-0.29308023-0.23856303$

$1 \quad 6.29300217-0.42930165-0.04878904$

$15.10381358-0.04932198-1.29542299$

$\begin{array}{lllll}1 & 4.89908294 & 0.56917997 & 0.34246016\end{array}$

$\begin{array}{lllll}6 & 4.44456320 & -1.54801161 & 0.12873577\end{array}$

$14.55297867-1.735826991 .20075271$

$14.89232563-2.40745933-0.37585277$

$6 \quad 2.95537942-1.50497574-0.21584314$

$1 \quad 2.52526241-2.474791860 .04159644$ 
$12.83898519-1.40277806-1.29946559$

$\begin{array}{lllll}6 & 2.15464804 & -0.39618020 & 0.48269853\end{array}$

$12.63093764-0.150124431 .43736807$

$1 \quad 1.16346465-0.774075580 .73694560$

$\begin{array}{llll}6 & 1.99649549 & 0.88406915 & -0.33921922\end{array}$

$1 \quad 1.556049690 .63091346-1.30902133$

$1 \quad 2.985144101 .28771901-0.56019972$

$\begin{array}{lllll}6 & 1.14133985 & 1.96651993 & 0.33696343\end{array}$

$\begin{array}{lllll}1 & 1.56891812 & 2.94848491 & 0.12636401\end{array}$

$\begin{array}{lllll}1 & 1.19658316 & 1.84224047 & 1.42315617\end{array}$

$\begin{array}{lllll}6 & -0.32540653 & 1.99074350-0.09447706\end{array}$

$\begin{array}{llll}1 & -0.37500338 & 2.18692965-1.17048435\end{array}$

$\begin{array}{lllll}1 & -0.82013041 & 2.83727077 & 0.39058299\end{array}$

$\begin{array}{lllll}6 & -1.11333365 & 0.72257337 & 0.21158800\end{array}$

$\begin{array}{lllll}1 & -1.03655597 & 0.49853044 & 1.28068354\end{array}$

$1-0.66618514-0.12701511-0.31157176$

$\begin{array}{llll}6 & -2.58149419 & 0.82067793 & -0.18309472\end{array}$

$1-2.653986801 .02741745-1.25572735$

$\begin{array}{lllll}1 & -3.03754762 & 1.67805221 & 0.32219337\end{array}$

$\begin{array}{llll}6 & -3.37775934 & -0.43692315 & 0.14107036\end{array}$

$1-3.31859345-0.633471661 .21647930$

$1-2.91171334-1.29763093-0.34968443$

$6-4.84110081-0.35409714-0.27595432$

$1-4.89831147-0.16892665-1.35221374$

$\begin{array}{lllll}1 & -5.30302586 & 0.51201427 & 0.20601701\end{array}$

$\begin{array}{llll}6 & -5.63028379-1.61076056 & 0.06757325\end{array}$

$1-5.61417215-1.799855711 .14245186$

$1-5.20580685-2.48633348-0.42705711$

$1-6.67250508-1.52517072-0.24042814$ energy $\quad 4.49756$

Symmetry point group: C1 moment of inertia 2.2816

Cartesian coordinate of structure 95

$6 \quad 2.249888012 .16478779-0.52493035$

$1 \quad 1.966095513 .18954172-0.28275410$

$1 \quad 2.345648462 .09527233-1.61028274$

$1 \quad 1.427151631 .51650305-0.22705351$

$\begin{array}{lllll}6 & 3.56335831 & 1.78701552 & 0.15519622\end{array}$

$\begin{array}{lllll}1 & 3.45412026 & 1.87447532 & 1.24025367\end{array}$

$14.314714262 .52626321-0.12983480$

$\begin{array}{lllll}6 & 4.09434186 & 0.38838332 & -0.18773668\end{array}$

$1 \quad 5.185231210 .40703691-0.14178295$

$13.846657880 .15287805-1.22651843$

$\begin{array}{lllll}6 & 3.62540417 & -0.74017764 & 0.73477179\end{array}$

$13.97930899-0.52303047 \quad 1.74661848$

$14.12527034-1.664516690 .43009043$

$\begin{array}{llll}6 & 2.12030363 & -0.99535210 & 0.79082936\end{array}$

$11.61433149-0.103727021 .16671250$ 


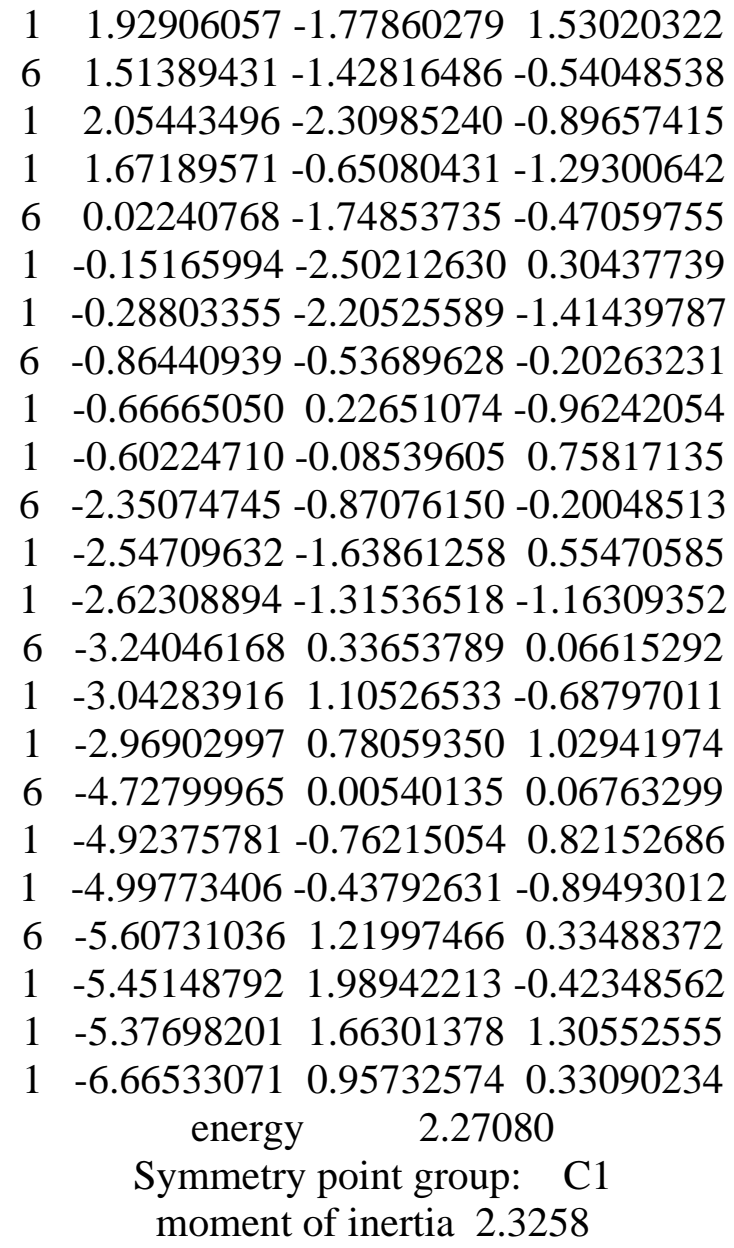

\section{References}

1- Adamson AW, Gast AP (1997) Physical chemistry of surface, $6^{\text {th }}$ edn. Wiley, New York

2- Gun’ko VM, Turov VV (2013) Nuclear Magnetic Resonance Studies of Interfacial Phenomena, CRC Press, Boca Raton

3- S. S. Sazhin, Droplets and Sprays (Springer, London, 2014). 